Pacific Journal of Mathematics

OMOTOPY THEORETIC CONSEQUENCES OF N. LEVITT'S
OBSTRUCTION THEORY TO TRANSVERSALITY FOR

GREGORY WAYNE BRUMFIEL AND JOHN W. MORGAN 


\title{
HOMOTOPY THEORETIC CONSEQUENCES OF N. LEVITT'S OBSTRUCTION THEORY TO TRANSVERSALITY FOR SPHERICAL FIBRATIONS
}

\author{
Gregory W. Brumfiel aNd John W. Morgan
}

The main goal of this paper is a detailed analysis of the problem of imposing a topological bundle structure on a spherical fibre space over a simply connected base. The method involves a careful study of the notion of fibre homotopy transversality due to $\mathrm{N}$. Levitt. The point is, a topological disc bundle satisfies strong transversality properties for maps from manifolds to the associated Thom space. These properties can be formulated at least for spherical fibre spaces. Thus, obstructions to transversality can be interpreted as obstructions to imposing a topological bundle structure on a spherical fibre space. It turns out that over a simply connected base the obstructions to transversality coincide exactly with the obstructions to a topological structure.

The obstructions to transversality for a spherical fibre space $\xi$ can be interpreted as obstructions to a deformation of the identity map on the Thom space $T \xi$ to a certain subcomplexes $W \xi$. The fibre of the map $W \xi \rightarrow T \xi$ isa sp ace with a suitable iterated loop space homotopy equivalent to $G / T O P$. The total obstruction to transversality becomes the obstruction to a $K O \otimes Z[1 / 2]$ orientation of the Thom space $T \xi$, mixed with certain cohomology classes of $T \xi, \tilde{\mathscr{L}} \in H^{4 *+1}\left(T \xi, Z_{(2)}\right)$ and $\widetilde{\mathscr{K}} \in H^{* *-1}(T \xi, Z / 2)$. These obstructions are then also interpretable as the obstructions to lifting in the fibration sequence $G / T O P \rightarrow B S T O P \rightarrow B S G$.

In this introduction, we give a rather detailed outline of our results and describe the relationship with work of others, particularly, N. Levitt [10], F. Quinn [18], and L. Jones [8]. Suppose that $\pi: \xi \rightarrow B \xi$ is a spherical fibre space, with Thom space $T \xi$, and let $f: M \rightarrow T \xi$ be a map from a $P L$ manifold to $T \xi$. The primary question is, very roughly, when can one $\operatorname{deform} f$, so that $f^{-1}(B \xi) \subset M$ is a Poincaré duality space?

If $\xi \rightarrow B \xi$ is a (block) $P L$ sphere bundle, the answer is always. In fact, in this case we may deform $f$ so that $L=f^{-1}(B \xi) \subset M$ is a $P L$ submanifold of $M$, with a tubular neighborhood $V=f^{-1}(D \xi)$, where $D \xi$ is the associated $P L$ block bundle of $\xi$. $V$ is a block bundle over $L$ and $f$ induces a bundle map 


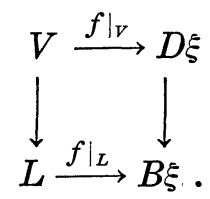

Note that $V$ itself is Poincaré duality space, homotopy equivalent to $L$, and the inclusion of the boundary $\partial V \subseteq V$ is, up to homotopy, a spherical fibration.

These considerations lead one to a correct definition of transversality if $\pi: \xi \rightarrow B \xi$ is only a spherical fibration. Namely, let $\mathscr{C} \xi=$ $\xi \times I \bigcup_{\xi \times 0} B \xi$ be the mapping cylinder of $\pi: \xi \rightarrow \beta \xi$. (If $\xi$ is an honest sphere bundle, $\mathscr{M} \xi$ is the associated disc bundle $D \xi$.) Then $T \xi=$ $\mathscr{A} \xi \bigcup_{\xi} \mathscr{C} \xi$, where $\mathscr{C} \xi$ is the cone on $\xi$.

DEFinition (approximate). $f: M \rightarrow T \xi, M$ a closed manifold, is globally Poincaré transversal if the inclusion $f^{-1}(\xi) \subseteq f^{-1}(\mathscr{M} \xi)$ is a spherical fibration, induced by $f$ from $\xi G \mathscr{M} \xi$.

If $\xi^{q}$ has fibre $S^{q-1}$ and $M$ has dimension $q+i$, the definition implies that $f^{-1}(\mathscr{C l} \xi)$ is a Poincaré duality space (PD space) of formal dimension $i$. There is an analogous definition if the manifold $M$ has a boundary; in this case, $\left(f^{-1}(\mathscr{M} \xi), f^{-1}(\mathscr{M} \xi) \cap \partial M\right)$ is a $\mathrm{PD}$ space with boundary.

Now the theory of Levitt states that if $B \xi^{q}$ is 1 -connected, $q \geqq 3$, there is an Eilenberg-MacLane obstruction theory, with obstructions in $H^{q+i+1}\left(M, Q_{i}\right)$, to deforming $f: M \rightarrow T \xi^{q}$ to a transversal map, where (at least if $i>4$ ) the group $Q_{i}$ is isomorphic to the surgery obstruction group

$$
P_{i}=Z, 0, Z / 2,0 \quad \text { as } \quad i \equiv 0,1,2,3(\bmod 4) .
$$

Quinn's theory of surgery on PD spaces implies that if $M$ has dimension $q+j+1$, there is a single obstruction, in $P_{j}$ to deforming $f: M \rightarrow T \xi^{q}$ to a transversal map. This discrepancy is easy to explain. By "transversal map," Levitt means that $M$ is triangulated such that for each simplex $\Delta \subset M,\left.f\right|_{4}: \Delta \rightarrow T \xi^{q}$ is Poincaré transversal, whereas Quinn uses "transversal map" in the global sense of the definition above. One problem which interested us then was whether we could identify a global, "top" obstruction in the context of Levitt's cell-by-cell obstruction theory. We do define a global obstruction to transversality, using the Levitt theory, much as one picks out the surgery obstruction from all the invariants of a normal map. However, the methods of this paper only enable us to prove that this top obstruction is the obstruction to cobording (rather than homo- 
toping) a map $f: M \rightarrow T \xi$ to a transversal map. Our obstruction does agree with Quinn's, however, and thus from his theory it follows that it is the obstruction to homotoping $f$ to a globally transversal map.

A second problem was to extend the results of Levitt and Morgan [11] relating Poincaré transversality and topological structures on a spherical fibration $\xi$. They prove (roughly) that if the base space $B \xi$ is 4 -connected and if every map $f: M \rightarrow T \xi$ can be made Poincaré transversal, then $\xi$ admits a topolgical structure. In fact, there is a $1-1$ correspondence between topological structures on $\xi$ and "Poincaré transversality structures" on $\xi$. We extend these results to 1-connected base spaces. A homotopy theoretic reformulation of these geometric results gives that at odd primes $p$, a topological structure on $\xi$ is equivalent to a $K O \otimes \boldsymbol{Z}_{(p)}$-theory orientation of $T \xi$, (this is a well-known theorem of Sullivan [23]) and at $p=2$, a topological structure is equivalent to null-homologies of certain stable cohomology characteristic classes of $\xi, \tilde{\mathscr{K}}(\xi) \in H^{4 *-1}(B \xi, Z / 2)$ and $\tilde{\mathscr{L}}(\xi) \in H^{4^{*}+1}\left(B \xi, \boldsymbol{Z}_{(2)}\right)$. Similar results were proved by Quinn $\left[Q_{2}, Q_{3}\right]$; the $p=2$ results have also been proved by Madsen and Milgram [12] and Jones [8]. A very precise definition of the classes $\tilde{\mathscr{K}}(\xi)$ and $\tilde{\mathscr{L}}(\xi)$ and a detailed study of their properties accounts for much of the bulk of the present paper.

Our third main problem was to understand the relations between the top obstruction to Poincare transversality in dimensions $4 n+1$ and the index of Poincaré duality spaces. We prove, for example, that the obstruction $\tilde{\mathscr{L}}(\xi) \in H^{4^{*}+1}\left(B \xi, \boldsymbol{Z}_{(2)}\right)$ to a topological structure (or to Poincaré transversality, since these are the same) is the Bockstein (for the coefficient sequence $0 \rightarrow \boldsymbol{Z}_{(2)} \rightarrow \boldsymbol{Z}_{(2)} \rightarrow \boldsymbol{Z} / 8 \rightarrow 0$ ) of a characteristic class $l(\xi) \in H^{4^{*}}(B \xi, Z / 8)$. Among the properties of $l(\xi)$ is this: if $K^{4 n}$ is a $4 n$-dimensional PD space and $\nu_{K}$ is its stable normal spherical fibration, then $\left.\left\langle l\left(\nu_{K}\right)\right\rangle,[K]\right\rangle=\operatorname{index}(K)(\bmod 8)$. (The existence of such a class was shown by D. Frank, also using Levitt's theory of transversality. We gives additional properties of $l(\xi)$ which characterize it uniquely.) The result $\tilde{\mathscr{L}}(\xi)=\beta l(\xi)$ is intimately related to the theorem of Morgan and Sullivan [15] that there is a $\boldsymbol{Z}_{(2)}$ characteristic class $L(\xi) \in H^{4^{*}}\left(B \xi, \boldsymbol{Z}_{(2)}\right)$ of topulogical bundles $\xi$, which measures the index in $Z$ of topological manifolds. $L$ is defined using topological transversality, and reduces modulo 8 to our class $l(\xi)$. The connection is clear: if one has enough transversality, the $\boldsymbol{Z} / 8$ index class $l(\xi)$ lifts to a $\boldsymbol{Z}_{(2)}$ index class $L(\xi)$, which gives a homology of $\tilde{\mathscr{L}}(\xi)=\beta l(\xi)$ to 0 .

We now begin a more detailed outline of the individual sections of the paper. Chapter I, consisting of the first four sections, contains 
the geometric machinery and results of the paper. In $\S 1$ we develop a modified version of the Levitt obstruction theory. Namely, we strengthen the definition of a Poincaré transversal $q+2$ simplex, $f: \Delta^{q+2} \rightarrow T \xi^{q}$, by adding the condition that $f^{-1}(\mathscr{L} \xi) \subset \Delta^{q+2}$ have the homotopy type of a 2-manifold with boundary. In other dimensions, the definition is unchanged. It is an old conjecture [24] that all 2dimensional $\mathrm{PD}$ spaces are homotopy equivalent to manifolds. If this is true, our obstruction theory agrees with Levitt's. The advantage of the stronger definition is that it is possible to compute the obstruction groups in all dimensions, whereas Levitt's low dimensional obstruction groups were unknown.

The fundamental lemma in $\S 1$ is the following local result.

THEOREM A. Suppose that the spherical fibration $\xi^{q}$ admits a $P L$ structure, $q \geqq 3, \pi_{1}\left(B \xi^{q}\right)=0$, and let $f: D^{q+i+1} \rightarrow T \xi^{q}$ be a map such that $\left.f\right|_{\partial D}: S^{q+i} \rightarrow T \xi^{q}$ is Poincaré transversal. Assume further that if $i=2, f^{-1}(\mathscr{C} \xi) \cap S^{q+i}$ is homotopic to a 2-manifold. Then there is a well-defined obstruction $\alpha(f) \in P_{i}$ such that $f$ deforms rel $\partial D$ to a Poincaré transversal map if and only if $\alpha(f)=0$.

$\alpha(f) \in P_{i}$ is the surgery obstruction of a degree one normal map $L^{\prime} \rightarrow L$, where $L^{\prime}$ is an $i$-dimensional $P L$ manifold and $L$ is an $i$ dimensional PD space. Namely, $L=f^{-1}(\mathscr{L} \xi) \cap S^{q+i}$, and $L^{\prime} \subset L$ is obtained by deforming $f: D^{q+i+1} \rightarrow T \xi^{q}$ slightly to a $P L$ transversal map, keeping $f^{-1}(\mathscr{C} \xi)$ setwise fixed.

Using the local result, we set up an obstruction theory, with obstructions in $H^{q+i+1}\left(M, P_{i}\right)$, to deforming a map $f: M \rightarrow T \xi^{q}$ to a strongly transversal map, where $\xi^{q}$ is any spherical fibre space with simply connected base. The idea is, we may cover the base $B \xi^{q}$ by simply connected, open sets $U_{\alpha}$, all containing the base point such that $\left.\xi^{q}\right|_{U_{\alpha}}$ admits a $P L$ structure. This induces a cover $\left\{V_{\alpha}\right\}$ of $T \xi^{q}$, and our first step is to triangulate $M$ such that for each simplex $\Delta \subset M, f(\Delta) \subset V_{\alpha}$, some $\alpha$. If $f$ is strongly transversal on the $q+i$ skeleton of $M$, the local result enables us to define an obstruction cocycle in $C^{q+i+1}\left(M, P_{i}\right)$, which vanishes if and only if $f$ deforms rel the $q+i$ skeleton to a map which is strongly transversal on the $q+i+1$ skeleton.

In $\S 2$ we reformulate the obstruction theory. Specifically, let $W_{\xi^{q}}$ be the subcomplex of the singular complx of $T \xi^{q}$ consisting of simplexes $f: \Delta^{q+i} \rightarrow T \xi^{q}$ which are Poincaré transversal, with $f\left(\Delta^{q+i}\right) \subset V_{\alpha}$, some $V_{\alpha} \subset\left\{V_{\alpha}\right\}$, and with $f^{-1}(\mathscr{C} \xi) \subset \Delta^{q+i}$ homotopic to a 2-manifold if $i=2$. As a corollary of the obstruction theory of $\S 1$, we deduce

$$
\pi_{q+i+1}\left(T \xi^{q}, W_{\xi^{q}}\right) \stackrel{\sim}{\longrightarrow} P_{i} .
$$


In fact, the obstructions to deforming $f: M \rightarrow T \xi^{q}$ to a strongly transversal map can be interpreted as the obstructions to lifting $f$ to a map $\tilde{f}$ in the diagram

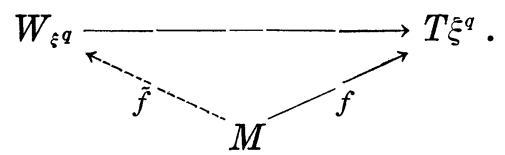

The space $W_{\xi^{q}}$ was also studied by Levitt, without the extra condition on $q+2$ simplexes. Our space $W_{\xi^{q}}$ is more natural for studying topological structures on $\xi^{q}$, since if $\xi^{q}$ is a topological bundle, topological transversal simplexes $\Delta^{q+i} \rightarrow T \xi^{q}$ clearly belong to $W_{\xi}$. On the other hand, Levitt's original space was more closely related to Poincaré duality cobordism, the problem he was studying at the time.

Let $F_{\xi^{q}}$ be the fibre of the natural map $W_{\xi^{q}} \rightarrow T \xi^{q}$. In $\S 2$ we also define very powerful homomorphisms on the $\boldsymbol{Z} / n$ bordism of $F_{\xi}, n \geqq 0$,

$$
\mathcal{O}_{n}: \Omega_{q+\imath}\left(F_{\xi}, Z / n\right) \longrightarrow P_{\imath} \otimes Z / n \text {. }
$$

(A $Z / n$ manifold is an oriented manifold $M$, together with an orientation preserving isomorphism of the boundary of $M$ with $n$ disjoint copies of a manifold $\delta M, \phi: \amalg_{n} \delta M \stackrel{\sim}{\rightarrow} \partial M$. A $Z / 0$ manifold is thus a closed, oriented manifold. $\delta M$ is called the Bockstein of $M$; a $Z / n$ bordism element must map the $n$ copies of the Bockstein equivariantly.) To define $\mathcal{O}_{n}$, we first give a geometric interpretation of bordism elements of $F_{\xi} q$. Namely, $\varphi: M \rightarrow F_{\xi} q$ corresponds to a strongly transversal map $f: M \rightarrow T \xi^{q}$, together with a homotopy $F: M \times I \rightarrow T \xi^{q}$ from $F_{0} \equiv f$ to $F_{1} \equiv \infty, \infty \in T \xi^{q}$ the cone point. Using the obstruction theory of $\S 1$, we show that if $M^{q+i}$ is $Z / n$ manifold, there is a well-defined obstruction $\mathscr{O}_{n}[M, \varphi] \in P_{i} \otimes Z / n$ to deforming $F: M \times$ $I \rightarrow T \xi^{q}, \operatorname{rel} M \times \partial I$, to a globally transversal map. $\mathscr{O}_{n}[M, f]$ is (not surprisingly) the surgery obstruction of a degree one normal map $L^{\prime} \rightarrow L$, where $L^{\prime}$ is a $Z / n P L$ manifold and $L$ is a $Z / n$ PD space.

To see how these surgery problems arise, suppose first that $\xi^{q}$ admits a $P L$ structure. Shift $F: M \times I \rightarrow T \xi^{q}$ slightly to a $P L$ transversal map, $G$, keeping $F^{-1}\left(\mathscr{C} \xi^{q}\right)$ setwise fixed. Then $L^{\prime}=G^{-1}\left(B \xi^{q}\right) \cap$ $M \times\{0\} \subset G^{-1}\left(\mathscr{L} \xi^{q}\right) \cap M \times\{0\}=F^{-1}\left(\mathscr{C l} \xi^{q}\right) \cap M \times\{0\}=L$ is a degree one normal map.

If $\xi^{q}$ is an arbitrary special fibre space, we use the obstruction theory to produce a normal map. Namely, since $H^{*}(M \times I, M \times\{0\})=0$, we may deform $F$ rel $M \times\{0\}$ to a strongly transversal map $G: M \times$ $I \rightarrow T \xi^{q}$, such that $\left.G\right|_{M \times\{1\}}: M \times\{1\} \rightarrow T\left(\left.\xi^{q}\right|_{U}\right)$, where $U$ is a contractible neighborhood of the basepoint of $B \xi^{q}$. (See Chapter I for details.) 
Moreover, $\left.G\right|_{M \times\{1\}}: M \times\{1\} \rightarrow T\left(\left.\xi^{q}\right|_{U}\right)$ is homotopic to the constant map $\infty, \infty \in T \xi^{q}$, since $G$ is the deformation of $F$. Now $\left.\xi^{q}\right|_{U}$ admits a $P L$ structure since $U$ is contractible, hence we apply the construction of the paragraph above to $\left.G\right|_{M \times\{1\}}$ to obtain a normal map.

We point out that if $\xi^{q}$ admits a $P L$ structure, the normal map produced by this general construction is cobordant to the normal map produced directly from $P L$ transversality. Thus the surgery obstruction is well defined.

The resulting homomorphisms $\mathcal{O}_{n}: \Omega_{q+i}\left(F_{\xi}, \boldsymbol{Z} / n\right) \rightarrow P_{i} \otimes Z / n$ satisfy properties essentially identical to the properties of the classical surgery obstruction homomorphisms of Sullivan;

$$
S_{n}: \Omega_{i}\left(G / T O P, Z_{n} / n\right) \longrightarrow P_{i} \otimes Z / n .
$$

The most important properties are (i) the composition

$$
\pi_{q+i}\left(F_{\xi^{q}}\right) \stackrel{i}{\longrightarrow} \Omega_{q+i}\left(F_{\xi^{q}}\right) \stackrel{\ominus}{\longrightarrow} P_{i}
$$

is an isomorphism and (ii) $\mathscr{O}_{n}$ satisfies a multiplicative formula like those of [15] with respect to the index; that is, if $\varphi: M^{q+i} \rightarrow F_{\xi^{q}}$ is a $\boldsymbol{Z} / n$ bordism element and $N^{j}$ is a $\boldsymbol{Z} / n$ manifold, then

$$
\mathcal{O}_{n}\left[M^{q+i} \otimes N^{j}, \varphi \pi_{1}\right]=\mathcal{O}_{n}\left[M^{q+i}, \varphi\right] \cdot \operatorname{index}\left(N^{j}\right) .
$$

(See [15] for a definition and properties of the "product" $M^{q+i} \otimes N^{j}$ of $\boldsymbol{Z} / n$ manifolds.)

Just as Sullivan showed that the surgery obstruction homomorphisms $S_{n}: \Omega_{i}(G / T O P, Z / n) \rightarrow P_{i} \otimes Z / n$ completely determine the homotopy type of $G / T O P$, we show in $\S 5$ that the obstructions $\mathcal{O}_{n}: \Omega_{q+i}\left(F_{\xi^{q}}, Z / n\right) \rightarrow$ $P_{i} \otimes Z / n$ completely determine the homotopy type of $F_{\xi q}$. The result is that $F_{\xi^{q}}$ is a copy of $G / T O P$, shifted $q$-dimensions. (We will discuss this further below.)

These results of $\S 2$ were known earlier to Levitt and Morgan [11] for the $(q+4)$-connected cover of $F_{\xi q}$. The results of this paper simply extend their results, thanks to the stronger definition of transversality in dimension $q+2$.

In $\S 3$ we define obstructions to global transversality, up to cobordism. The main idea is that, in the stable range, $i \ll q$, there is an isomorphism

$$
\Omega_{q+i+1}\left(W_{\xi^{q}}, F_{\xi^{q}}, Z / n\right) \stackrel{\sim}{\longrightarrow} \widetilde{\Omega}_{q+i+1}\left(T \xi^{q}, Z / n\right) .
$$

Composing the $\mathcal{O}_{n}$ of $\S 2$ with the boundary homomorphism for the pair $W_{\xi q}, F_{\xi q}$, thus defines homomorphisms

$$
s_{n}: \Omega_{q+i+1}\left(T \xi^{q}, Z / n\right) \longrightarrow P_{i} \otimes Z / n,
$$


for $i \ll q, q \geqq 3$. We prove

Theorem B. If $i$ is even (resp. $i$ odd) and $f: M^{q+i+1} \rightarrow T \xi^{q}$ is a $\boldsymbol{Z} / n$ manifold, then $[M, f]$ is $\boldsymbol{Z} / n$-bordant to a globally transversal map if and only if $s_{n}[M, f]=0 \in P_{i} \otimes Z / n$ (resp. $s\left(\delta M,\left.f\right|_{\delta M}\right)=$ $\left.0 \in P_{i-1}\right)$.

As remarked earlier, Quinn [18] has sharper results about these obstructions to global transversality, namely that these are the obstructions to homotoping $f: M \rightarrow T \xi^{q}$ to a globally transversal map.

As a corollary of Theorem B, note that any map $f: M^{q+4 i} \rightarrow T \xi^{q}, M$ closed, is cobordant to globally transversal map $g: N^{q+4 i} \rightarrow T \xi^{q}$. Let $L^{4 i}=g^{-1}(\mathscr{C} \xi) \subset N^{q+4 i}$ be the associated $\mathrm{PD}$ space. We prove in $\S 3$ the following.

Lemma C. Index $\left(L^{4 i}\right) \in Z / 8$ in an invariant of the bordism class of $[M, f]$.

Since there is a null-homotopic PD transversal map $g: S^{q+4 i} \rightarrow$ $S^{q}=T e^{q}$, with $g^{-1}\left(\mathscr{C} e^{q}\right)=W^{4 i}$, where $W^{4 i}$ is the almost parallelizable Milnor manifold of index 8 , we see that the modulo 8 reduction of the index is the best invariant we can obtain from a transversal map $g: N^{q+4 i} \rightarrow T \xi^{q}$.

In $\S 8$ we generalize Lemma $\mathrm{C}$ to $\boldsymbol{Z} / 8$ manifolds. Not all $\boldsymbol{Z} / 8$ manifolds $f: M^{q+4 i} \rightarrow T \xi^{q}$ are cobordant to globally transversal maps, because of the obstruction $s\left[\delta M,\left.f\right|_{\delta M}\right] \in P_{4 i-2}=Z / 2$, provided by Theorem B. However

Lemma D. If $g: N^{q+4 i} \rightarrow T \xi^{q}$ is a globally transversal $Z / 8$ manifold, and $L^{4 i}=g^{-1}(\mathscr{L} \xi) \subset N^{q+4 i}$ is the associated $Z / 8$ PD space, then index $\left(L^{4 i}\right) \in \boldsymbol{Z} / 8$ is an invariant of the $\boldsymbol{Z} / 8$ bordism class of $[N, g]$.

This is the main result we need to define $Z / 8$ characteristic class of spherical fibrations, which measures the index $(\bmod 8)$ of PD spaces. (We will discuss this further below.)

In $\S 4$, we extend the main result of Levitt and Morgan [11] concerning the equivalence of topological structures on bundles $\xi^{q}$ over 4-connected base spaces and liftings $l$ in the diagram

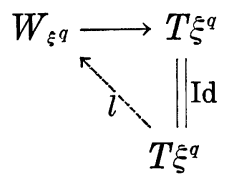


Our result is

THEOREM E. If $\xi^{q}$ is a spherical fibration over a simply connected base, $q \geqq 3$, then there is a natural bijection between equivalence classes of topological structures on $\xi^{q}$ and equivalence classes of lifting $l$ in the diagram

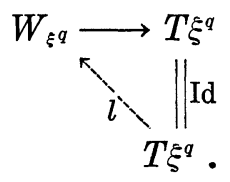

The proof breaks into two parts. The first part uses topological transversality to construct a lifting $l$ for a topological bundle $\xi^{q}$. This step is somewhat indirect since topological transversality cannot be assumed in codimension 4 . We use a "crossing with $C P(2)$ trick" to overcome this difficulty. The second part of the proof essentially uses the fact that $G_{q} / T O P_{q}$ and $F_{\xi q}$ are very closely related to establish that the map from topological structures on $\xi^{q}$ to liftings $l$ in the diagram is a bijective correspondence.

Chapter II, consisting of $\S \S 5$ through 7 , deals with homotopy theoretic consequences of the geometric results of Chapter I. In $\S 5$, we show how the homomorphisms $\mathcal{O}_{n}: \Omega_{q+i}\left(F_{\xi}, Z / n\right) \rightarrow P_{i} \otimes Z / n, n \geqq 0$, determine the homotopy type of $F_{\xi q}$. In particular, the 2-localization of $F_{\xi^{q}}$ is a product of Eilenberg-MacLane spaces,

$$
\left(F_{\xi}\right)_{(2)} \cong \prod_{i \geqq 1} K(Z / 2, q+4 i-2) \times K\left(Z_{(2)}, q+4 i\right) .
$$

The $p$-localization of $F_{\xi}, p$ odd, is a loop space of a connected cover of $B O_{(p)}$. Except for computing the low dimensional homotopy groups of $F_{\xi^{q}}$, these results were proved in [11] by the same techniques.

The 2-localization result is equivalent to establishing a cohomological formula for $\mathcal{O}: \Omega_{q+i}\left(F_{\xi^{q}}\right) \rightarrow P_{i}$ and for $\mathcal{O}_{2^{r}}: \Omega_{q+i}\left(F_{\xi^{q}}, Z / 2^{r}\right) \rightarrow$ $P_{i} \otimes Z / 2^{r}, r \geqq 1$. Namely, we prove

THEOREM F. There are unique cohomology classes

$$
\mathscr{C}=\sum_{\imath \geqq 1} \mathscr{K}_{q+4 i-2} \in H^{q+4^{*-2}}\left(F_{\xi}, Z / 2\right)
$$

and

$$
\mathscr{L}=\sum_{i \geqq 1} \mathscr{L}_{q+4 i} \in H^{q+4^{*}}\left(F_{\xi q}, \boldsymbol{Z}_{(2)}\right)
$$

such that given $f: M^{q+4 j-2} \rightarrow F_{q}, M a Z / 2$ manifold,

$$
\mathscr{O}_{2}[M, f]=\left\langle V^{2}(M) \cdot f^{*}(\mathscr{K}),[M]\right\rangle \in Z / 2
$$

and given $g: N^{q+4 j} \rightarrow F_{\xi}, N$ a closed or a $Z / 2^{r}$ manifold, 


$$
\begin{array}{r}
\mathcal{O}_{2}[M, f]=\left\langle L(N) \cdot g^{*}(\mathscr{L}),[N]\right\rangle+i\left\langle V S q^{1} V(\delta M) \cdot g^{*}(\mathscr{K}),[\delta N]\right\rangle \in Z / 2^{r} \\
(\in \mathbb{Z} \text { if } N \text { is closed) }
\end{array}
$$

where $i: Z / 2 \rightarrow Z / 2^{r}$ is the inclusion.

The formulae of Theorem $\mathrm{F}$ giving $\mathcal{O}_{2}: \Omega_{q+4 j-2}\left(F_{\xi q}, \boldsymbol{Z} / 2\right) \rightarrow \boldsymbol{Z} / 2$ in terms of $\mathscr{K}$ and giving $\mathcal{O}_{n}: \Omega_{q+4 j}\left(F_{\xi q}, Z / n\right) \rightarrow Z / n, n=0$ or $2^{r}$, in terms of $\mathscr{L}$ and $\mathscr{K}$, are identical to the formulae in [15] for the surgery obstruction homomorphisms $S_{n}: \Omega_{i}(G / T O P, Z / n) \rightarrow P_{i} \otimes Z / n$ in terms of cohomology classes

$$
\mathscr{K} \in H^{4 *-2}(G / T O P, \boldsymbol{Z} / n) \text { and } \mathscr{L} \in H^{4 *}\left(G / T O P, \boldsymbol{Z}_{(2)}\right) \text {. }
$$

This is no accident, since the $\mathscr{O}_{n}$ are defined as surgery obstructions.

The proof of Theorem $\mathrm{F}$ is essentially given in the Appendix, where we give a detailed discussion of how homomorphisms on the bordism of a space can be used to define cohomology classes. The version we present is due to Sullivan, [23], although variants of the idea have been used earlier, (for example, in Thom's definition of rational Pontrjagin classes for $P L$ manifolds). The classes $\mathscr{K}$ and $\mathscr{L}$ of Theorem $\mathrm{F}$ then define a homotopy equivalence

$$
\mathscr{K} \times \mathscr{L}:\left(F_{\xi^{q}}\right)_{(2)} \cong \prod_{i \geqq 1} K(Z / 2, q+4 i-2) \times K\left(Z_{(2)}, q+4 i\right) .
$$

From the cohomology exact sequence of the fibration $F_{\xi} \rightarrow W_{\xi} \rightarrow$ $T \xi$ (in the stable range) we see that the classes $\mathscr{K}$ and $\mathscr{L}$ in the cohomology of $F_{\xi}$ determine classes in the cohomology of $T \xi$. From the Thom isomorphism $\Phi: H^{*}(B \xi) \rightarrow \widetilde{H}^{*}(T \xi)$, we then get classes $\tilde{\mathscr{C}}(\xi) \in H^{4^{*-1}}(B \xi, \boldsymbol{Z} / 2)$ and $\tilde{\mathscr{L}}(\xi) \in H^{4^{*+1}}\left(B \xi, Z_{(2)}\right)$. Precisely, $\Phi(\tilde{\mathscr{K}}(\xi))=$ $\tau(\mathscr{K})$ and $\Phi(\tilde{\mathscr{L}}(\xi))=\tau(\mathscr{L})$ where $\tau: H^{*}\left(F_{\xi}\right) \rightarrow H^{*+1}(T \xi)$ is the transgression, in the stable range, of the fibration $F_{\xi} \rightarrow W_{\xi} \rightarrow T \xi$.

The classes $\tilde{\mathscr{K}}(\xi)$ and $\tilde{\mathscr{L}}(\xi)$ are stable characteristic classes for spherical fibrations. From the definition of the global transversality obstructions $s_{n}: \Omega_{q+i+1}\left(T \xi^{q}, Z / n\right) \rightarrow P_{i} \otimes Z / n$, in terms of

$$
\mathcal{O}_{n}: \Omega_{q+i}\left(F_{\xi}, Z / n\right) \longrightarrow P_{i} \otimes Z / n,
$$

we obtain cohomological formulae for the $s_{2^{r}}$ in terms of the classes $\Phi(\tilde{\mathscr{K}}(\xi))$ and $\Phi(\tilde{\mathscr{L}}(\xi))$ : (see Theorem $\mathrm{F}$ above)

$$
s_{2}\left[M^{q+4 j-1}, f\right]=\left\langle V^{2}(M) \cdot f^{*} \Phi(\tilde{\mathscr{\mathscr { K }}}(\xi)),[M]\right\rangle \in Z / 2
$$

if $f: M^{q+4 i-1} \rightarrow T \xi^{q}$ is a $Z / 2$ manifold, and

$$
\begin{aligned}
s_{2^{r}}\left[N^{q+4 j+1}, g\right]= & \left\langle L(N) \cdot g^{*} \Phi(\tilde{\mathscr{L}}(\xi)),[N]\right\rangle \\
& +i\left\langle V S q^{1} V(\delta N) \cdot g^{*} \Phi(\tilde{\mathscr{K}}(\xi)),[\delta N]\right\rangle \in Z / 2^{r}
\end{aligned}
$$


if $g: N^{q+4 j+1} \rightarrow T \xi^{q}$ is a $Z / 2^{r}$ manifold. Here $i: Z / 2 \rightarrow Z / 2^{r}$ is the inclusion. We thus regard $\tilde{\mathscr{K}}(\xi)$ and $\tilde{\mathscr{L}}(\xi)$ as obstructions to global Poincaré transversality.

The main theorem of $\S 6$ is that, at the prime $2, \tilde{\mathscr{K}}(\xi)$ and $\tilde{\mathscr{L}}(\xi)$ are the only obstructions to a topological structure on $\xi$. More precisely, let $\tilde{\mathscr{K}} \in H^{4^{*}-1}(B S G, Z / 2)$ and $\tilde{\mathscr{L}} \in H^{4^{*+1}}\left(B S G, Z_{(2)}\right)$ be the universal characteristic classes and let $i: X \rightarrow B S G_{(2)}$ be the inclusion of the fibre of the map

$$
\tilde{\mathscr{\mathscr { C }}} \times \tilde{\mathscr{L}}: B S G_{(2)} \longrightarrow \prod_{i \geqq 1} K(Z / 2,4 i-1) \times K\left(\boldsymbol{Z}_{(2)}, 4 i+1\right) .
$$

THEOREM G. There is a commutative diagram

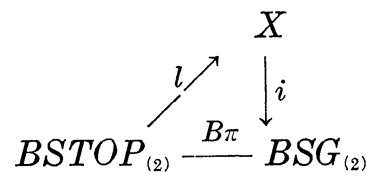

with $B \pi$ the natural map and $l$ a homotopy equivalence.

Thus a lifting of $\xi: Y \rightarrow B S G_{(2)}$ to $\tilde{\xi}: Y \rightarrow B S T O P_{(2)}$ is equivalent to null-cohomologies of the characteristic classes $\tilde{\mathscr{K}}(\xi)$ and $\tilde{\mathscr{L}}(\xi)$.

F. Quinn has proved a theorem very similar to Theorem G, [19], [20]. In fact, his thoerem is probably identical to Theorem G, but his definition of characteristic classes, corresponding to our $\tilde{\mathscr{K}}$ and $\tilde{\mathscr{L}}$, is rather abstract. I. Madsen and R.J. Milgram, [12], have also proved that $B S T O P_{(2)}$ is the fibre of a map

$$
B S G_{(2)} \longrightarrow \prod_{i \geqq 1} K(Z / 2,4 i-1) \times K\left(Z_{(2)}, 4 i+1\right) .
$$

Namely, they prove that $B(G / T O P)_{(2)}$ is a product of Eilenberg-MacLane spaces. The resulting cohomology classes of BSG are not identical to our $\tilde{\mathscr{K}}$ and $\tilde{\mathscr{L}}$, but they are closely related. At present, there is no geometric explanation of the connection between $B(G / T O P)$, the classifying space for G/TOP with the Whitney sum structure, and Poinaré transversality. The transversality theory seems more closely related to a second $H$-space structure on $G / T O P$. See also L. Jones [8] for a version of Theorem G.

In $\S 7$, we identify the suspensions of the cohomology classes $\tilde{\mathscr{K}}$ and $\tilde{\mathscr{L}}$; that is, $\sigma(\tilde{\mathscr{K}}) \in H^{4^{*}-2}(S G, \boldsymbol{Z} / 2)$ and $\sigma(\tilde{\mathscr{L}}) \in H^{4^{*}}\left(S G, \boldsymbol{Z}_{(2)}\right)$, where $\sigma: H^{*+1}(B S G) \rightarrow H^{*}(S G)$ is induced by the natural map $\sum S G \rightarrow$ $B S G$.

THEOREM H. $\sigma(\tilde{\mathscr{K}})=\pi^{*}(\mathscr{K})$ and $\sigma(\tilde{\mathscr{L}})=\pi^{*}(\mathscr{L})$, where $\pi$ : $S G \rightarrow G / T O P$ is the projection and $\mathscr{K} \in H^{4^{*-2}}(G / T O P, Z / 2)$ and 
$\mathscr{L} \in H^{4^{*}}\left(G / T O P, \boldsymbol{Z}_{(2)}\right)$ are the canonical surgery obstruction classes of [15].

Chapter III, which consists of $\S \S 8$ and 9 , gives the definition and properties of the $\boldsymbol{Z} / 8$ index class $l=1+l_{1}+l_{2}+\cdots \in H^{4^{*}}(B S G, \boldsymbol{Z} / 8)$. In $\S 8, l$ is defined in terms of a homomorphism on the $Z / 8$ bordism of $M S G, \phi: \Omega_{4^{*}}(M S G, Z / 8) \rightarrow Z / 8$. If $f: M \rightarrow M S G$ is a transversal map, $M$ a $Z / 8$ manifold, with $L=f^{-1}(B S G) \subset M$ the associated $Z / 8$ $\mathrm{PD}$ space, then

$$
\phi[M, f]=\operatorname{index}(L)-4\left\langle V S q^{1} V(M) \cdot f^{*} \Phi(\tilde{\mathscr{K}}),[M]\right\rangle \in \boldsymbol{Z} / 8 .
$$

Using results of the Appendix, we show that $\phi$ determines a class $l \in H^{4^{*}}(B S G, Z / 8)$ with

$$
\phi[M, f]=\left\langle L(M) \cdot f^{*} \Phi(l),[M]\right\rangle \in \boldsymbol{Z} / 8 .
$$

REMARK. Not all elements of $\Omega_{4 k}(M S G, Z / 8)$ are represented by transversal maps $f: M \rightarrow M S G$. In fact, the subgroup generated by transversal maps has index 2 , in each dimension $4 k$. To extend the above definition of $\phi$ to all of $\Omega_{4 k}(M S G, Z / 8)$, we need to choose a very specific non-transversal element $[K, \alpha]$, set $\phi[K, \alpha]=0$, and then prove that this is consistent with the definition of $\phi$ on transversal elements.

The class $l$ has the following properties

Theorem I. (i) If $L$ is a $Z / 8$ PD space and $\nu: L \rightarrow B S G$ is its normal fibration, then

$$
\left\langle\boldsymbol{\nu}^{*}(l),[L]\right\rangle=\operatorname{index}(L) \in \boldsymbol{Z} / 8 .
$$

(ii) The $Z / 2$ reduction of $l$ is $V^{2} \in H^{4^{*}}(B S G, Z / 2)$.

(iii) The class $l$ satisfies the Whitney sum formula

$$
\begin{array}{r}
l(\xi \times \eta)=l(\xi) \otimes l(\eta)+i\left(V S q^{1} V(\xi) \otimes \tilde{\mathscr{K}}(\eta)+\tilde{\mathscr{K}}(\xi) \otimes V S q^{1}(\eta)\right) \\
\in H^{4 *}(B \xi \times B \eta, Z / 8)
\end{array}
$$

where $i: H^{*}(, \boldsymbol{Z} / 2) \rightarrow H^{*}(, \boldsymbol{Z} / 8)$ is the natural coefficient map.

(iv) $\beta l=\tilde{\mathscr{L}} \in H^{4^{*+1}}\left(B S G, \boldsymbol{Z}_{(2)}\right)$, where $\beta$ is the Bockstein homomorphism of the coefficient sequence $0 \rightarrow \boldsymbol{Z}_{(2)} \rightarrow \boldsymbol{Z}_{(2)} \rightarrow \boldsymbol{Z} / 8 \rightarrow 0$.

Property (iv) relates the $Z / 8$ index class $l$ and transversality obstruction $\tilde{\mathscr{L}}$, and was discussed earlier. Here is a more precise statement of the relation between transversality and the index $(\bmod 8)$. Suppose $f=M^{q+4 i+1} \rightarrow T \xi^{q}$ is a $Z / n$ manifold such that $\left.f\right|_{\delta M}: \delta M \rightarrow T \xi^{q}$ is globally transversal. Let $L^{4 i}=f^{-1}\left(\mathscr{C} \xi^{q}\right) \cap \delta M$, a closed $P D$ space. If $f: M \rightarrow T \xi^{q}$ is itself transversal then index index $\left(L^{4 i}\right)=0$, since 
then $n \cdot L^{4 i}$ bounds a PD space, namely $f^{-1}\left(\mathscr{L} \xi^{q}\right) \subset M$. Thus index $(L)$ is an obstruction to cobording the map $f: M \rightarrow T \xi^{q}$ to a transversal map. We prove, in fact, that the transversality obstruction $s_{n}[M, f] \in$ $\boldsymbol{Z} / n$ of $\S 3$ always has order dividing 8 , and is exactly index $(L) \in Z / 8$. (In particular, if $n$ is odd, $s_{n} \equiv 0$.)

Theorem I(iii), the Whitney sum formula for $l$, is quite difficult. Its proof requires all of $\S 9$, the longest section of the paper. The term which measures deviation from multiplicativity, $i\left(V S q^{1} V(\xi) \otimes\right.$ $\left.\tilde{\mathscr{K}}(\eta)+\tilde{\mathscr{K}}(\xi) \otimes V S q^{1} V(\eta)\right)$, arises for the following reason. If we form a product of transversal maps $f$ and $g, f \times g: M^{p+a} \times N^{q+b} \rightarrow$ $T \xi^{p} \wedge T \eta^{q}$, with $a$ and $b$ odd, $a+b \equiv 0(\bmod 4)$, then clearly the index of the inverse image of $B \xi \times B \eta$ is zero, since this inverse is a product of odd dimensional $\mathrm{PD}$ spaces. However, if $f$ or $g$ is not transversal, it can occur that $f \times g$ is transversal with nonzero index for the inverse image of $B \xi \times B \eta$. This implies that, in $B S G \times$ $B S G$, the $l$ class can evaluate nontrivially on products of odd dimensional manifolds.

Since $\tilde{\mathscr{L}}=\beta l$, the Whitney sum formula for $l$ implies a Whitney sum formula for $\tilde{\mathscr{L}}$. We also establish in the paper a Whitney sum formula for the class $\tilde{\mathscr{K}}$, namely

THEOREM J.

$$
\tilde{\mathscr{K}}(\xi \times \eta)=\tilde{\mathscr{K}}(\xi) \otimes V^{2}(\eta)+V^{2}(\xi) \otimes \tilde{\mathscr{K}}(\eta) \in H^{4^{*-1}}(B \xi \times B \eta, Z / 2) .
$$

Using this formula and the fact that

$$
\sigma(\tilde{\mathscr{K}})=\pi^{*}(\mathscr{K}) \in H^{4^{*-2}}(S G, Z / 2), \pi: S G \longrightarrow G / T O P,
$$

(see Theorem $\mathrm{H}$ ). We deduce

COROLLARY K. $\tilde{\mathscr{K}}=V^{2} \cdot \mathscr{E}$, where $\mathscr{E}=\sum_{i \geqq 2} e_{2^{i}-1}$, and $e_{2^{i}-1} \in$ $H^{2^{i-1}}(B S G, Z / 2)$ is the unique primitive element with

$$
\sigma\left(e_{2^{i}-1}\right)=\pi^{*}\left(\mathscr{K}_{2^{i}-2}\right) \in H^{2^{i-2}}(S G, \boldsymbol{Z} / 2) .
$$

Clearly $\tilde{\mathscr{K}}(\xi) \equiv 0$ if and only if $\mathscr{E}(\xi) \equiv 0$, Thus, although $\tilde{\mathscr{K}}$ is nonzero in every dimension $4 i-1, i \geqq 1$, the "marrow" of the $\tilde{\mathscr{K}}$-class is concentrated in dimensions $2^{i}-1$. There is evidence that this class $\mathscr{E}$ is the same as the class defined by D. Ravenal in terms of twisted secondary operations [21]. $\quad \mathscr{E}$ is definitely equal to the class in dimensions $4^{*}-1$ produced by Madsen and Milgram's result that $B(G / T O P)_{(2)} \cong \Pi_{i \geqq 1} K(Z / 2,4 i-1) \times K\left(Z_{(2)}, 4 i+1\right)$.

Chapter IV, which consists of $\S \S 10$ and 11, is concerned with 
giving homotopy theoretic definitions of the classes $\tilde{\mathscr{K}}$ and $\tilde{\mathscr{L}}$. Section 10 extends slightly recent work of W. Browder, [1]. Namely, Browder observed that if a sphere $\alpha: S^{q+4 i-1} \rightarrow T \xi^{q}$ is transversal, then $\alpha$ lifts to $\tilde{\alpha}: S^{q+4 i-1} \rightarrow T \xi^{q}\left\langle v_{2 i}\right\rangle$, where $K(Z / 2,2 i-1) \rightarrow B \xi\left\langle v_{2 i}\right\rangle \rightarrow B \xi$ is the fibration which kills the $\mathrm{Wu}$ class $v_{2 i}(\xi) \in H^{2 i}(B \xi, Z / 2)$, and $\xi^{q}\left\langle v_{2 i}\right\rangle$ is the bundle $\xi^{q}$ lifted to $B \xi\left\langle v_{2 i}\right\rangle$. $\alpha$ lifts to $\tilde{\alpha}$ because if $K^{4 i-1}$ is a PD space of dimension $4 i-1, v_{2 i}\left(K^{4 i-1}\right)=0$. Browder then proved that the homomorphism

$$
\rho_{*}: \pi_{q+4 i-1}(T \xi) \longrightarrow \pi_{q+4 i-1}\left(T \xi / T \xi\left\langle v_{2 i}\right\rangle\right)
$$

has image $\left(\rho_{*}\right) \subset Z / 2$ and that $\rho_{*}(\alpha)$ agrees exactly with the Levitt obstruction to transversality on spheres $\alpha: S^{q+4 i-1} \rightarrow T \xi^{q}$. Moreover he showed how $\rho_{*}(\alpha) \in Z / 2$ is computed as an explicit functional cohomology operation.

We observe that since $\widetilde{\Omega}_{*}(T \xi, Z / 2) \cong \pi_{*}(T \xi \wedge M O \wedge R P(2))$, we can use Browder's functional operation on homotopy elements in the Thom space $T \xi \wedge M O \wedge R P(2)$ to compute $s_{2}: \Omega_{q+4 i-1}\left(T \xi^{q}, Z / 2\right) \rightarrow Z / 2$. Since $s_{2}$ defines $\tilde{\mathscr{K}}(\xi)$, we obtain an alternate definition of $\tilde{\mathscr{K}}(\xi)$, purely in terms of homotopy theoretical constructions. We use this functional operation definition, in fact, to prove the Whitney sum formula of Theorem $\mathrm{J}$ for the class $\tilde{\mathscr{K}}$.

In $\S 11$, we give a somewhat similar homotopy theoretic definition of the $Z / 8$ index homomorphism $\Omega_{q+4 i}\left(T \xi^{q}\right) \rightarrow Z / 8$ (see Theorem B and Lemma C). Since this determines the transversality obstruction homomorphisms $s_{n}: \Omega_{q+4 i+1}\left(T \xi^{q}, \boldsymbol{Z} / n\right) \rightarrow \boldsymbol{Z} / n$, (see the discussion of Theorem I(iv)), we obtain a homotopy theoretical definition of the class $\tilde{\mathscr{L}}(\xi)$.

The paper concludes with an Appendix describing how to define $\boldsymbol{Z} / 2, Q, \boldsymbol{Z}_{(2)}$, or $\boldsymbol{Z} / 2^{r}$ cohomology classes of a space $X$, in terms of homomorphisms on the bordism of $X$ with various coefficients. The cases $\boldsymbol{Z} / 2, Q$, and $\boldsymbol{Z}_{(2)}$ are discussed thoroughly in [23] and [15]; the $\boldsymbol{Z} / 2^{r}$ case is implicit in [15] and [13]. We include the Appendix primarily for completeness.

\section{CHAPTER I}

1. The obstruction theory. In this section we will set up an obstruction theory for the question of putting a manifold fiber homotopy transverse regular to the base of a spherical fiber space. This obstruction theory is just a recasting of the one originally developed in [10] except that we make different technical assumptions about the class of maps which we will consider. This allows us to avoid the difficulties encountered in the original theory. We will prove that if $f: D^{q+k} \rightarrow T\left(\xi^{q}\right)$ is f.h.t. on the boundary and $\xi^{q}$ admits a $P L$ 
structure (though none is preferred) then there is one obstruction $\alpha \in P_{k-1}$ to shifting $f$ relative to the boundary to a transversal map. $\alpha$ is the surgery obstruction of the following normal map. Put a $P L$ structure on $\xi^{q}$ and shift $f$ slightly to be $P L$ transverse. Let $Y^{q}=f^{-1}$ (0-section). Then $\partial Y^{q} \subset f^{-1}\left(M_{\xi}\right) \cap S^{q+k-1}$ is a degree one normal map. We show that $\alpha$ is well defined independent of the $P L$ structure we put an $\xi^{q}$.

Using this local result we are able to develop an obstruction theory. Namely, let $f: M^{q+k} \rightarrow T\left(\xi^{q}\right)$. Suppose we are given a triangulation of $M$ such $f$ (any simplex) is contained in the Thom space of a bundle admitting a $P L$ structure. Then we have an obstruction theory to inductively deforming $f$ over the skeleta of $M$ to be f.h.t. The obstructions lie in $H^{*}\left(M ; P_{*-q-1}\right)$. The rest of this paper involves ramifications of this theory.

We begin by recalling the definition of fiber homotopy transverse regular (denoted f.h.t.). Let $E\left(\xi^{q}\right) \stackrel{\pi}{\longrightarrow} X$ be a spherical fiber space (i.e., the homotopy theoretic fiber of $\pi$ is $S^{q-1}$ ). Let $M_{\xi}$ be the mapping cylinder of $\pi$ and let

$$
T\left(\xi^{q}\right)=M_{\xi_{E(\xi q)}} \operatorname{cone}\left(E\left(\xi^{q}\right)\right)
$$

$T\left(\xi^{q}\right)$ is the Thom space of $\xi^{q} . \quad E\left(\xi^{q}\right) \subset T\left(\xi^{q}\right)$ has a trivial line bundle as normal bundle

DEFINITION 1.1. $f: M^{q+n} \rightarrow T\left(\xi^{q}\right), M$ a closed $P L$ manifold, is globally fiber homotopy transverse to $X$, (f.h.t.) if and only if

(1) $f$ is $P L$ transverse to $E\left(\xi^{q}\right) \subset T\left(\xi^{q}\right)$ and

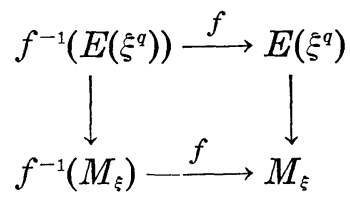

is a map of spherical fiber spaces. Of course, the spherical fibration on the right is canonically equivalent to $E\left(\xi^{2}\right) \stackrel{\pi}{\longrightarrow} X$.

From this definition it follows easily that $f^{-1}\left(M_{\xi}\right)$ is a Poincaré duality space, (PD space) of formal dimension $n$ (see [24] for a definition), and that its normal bundle in $M^{q+n}$ is induced from $\xi$ by $f$ restricted to $f^{-1}\left(M_{\xi}\right)$.

If $M^{q+n}$ is a manifold with boundary, we require 


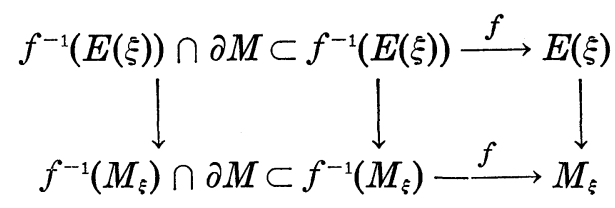

to be maps of spherical fiber spaces. It follows in this case that $\left(f^{-1}\left(M_{\xi}\right), f^{-1}\left(M_{\xi}\right) \cap \partial M\right)$ is a Poincaré duality pair (PD pair), of formal dimension $n$.

We will use a stronger, local concept of transversality.

Definition 1.2. Let $M^{q+n}$ be a $P L$ manifold with a given combinatorial triangulation, and let $\xi^{q}$ be as before. We say that $f: M^{q+n} \rightarrow T\left(\xi^{q}\right)$ is strongly f.h.t. if and only if

(1) $f \mid \Delta^{i}: \Delta^{i} \rightarrow T\left(\xi^{q}\right)$ is f.h.t. for each simplex $\Delta^{i}$ in the triangulation of $M$, and

(2) $\left(f^{-1}\left(M_{\xi}\right) \cap \Delta^{q+2}, f^{-1}\left(M_{\xi}\right) \cap \partial \Delta^{q+2}\right)$ is then a 2 dimensional PD pair; we require that this pair be homotopy equivalent to a $P L 2$-manifold with boundary.

This definition requires some explanation. First, there is a great difference between a map of a manifold into a spherical fibration being f.h.t. and strongly fiber homotopy transverse, even if we ignore the extra low dimensional condition. We will see later that the former is analogous to requiring a map between simply connected manifolds to have 0 surgery obstruction (i.e., be normal cobordant to a homotopy equivalence), whereas the latter is analogous to requiring all the splitting invariants of the map to be 0 . (Which of course makes it normally cobordant to a homeomorphism.) The analogy is easily understood from the remark that if $f ; M \rightarrow T(\xi)$ is strongly transverse then so is $f$ restricted to any simplicial singular submanifold of $N$ (i.e., $N \stackrel{r}{\longrightarrow} M \stackrel{f}{\longrightarrow} T(\xi)$ for $N$ a manifold and $r$ simplicial). Secondly, the condition on the $(q+2)$-simplicies is to circumvert our lack of understanding of $G$-framed PD bordism in dimension 2. We will reduce problems about f.h.t. on $(q+k)$-simplicies to problems about $G$-framed PD bordism in dimension $k$. We will be able to handle the problems about $G$-framed PD bordism except in dimension 2 where we will use the extra hypothesis.

The main theorem of this section is the following.

THEOREM 1.3. If $\xi^{a} \stackrel{\pi}{\longrightarrow} X$ is a spherical fibration, $\pi_{1}(X)=0$, $q \geqq 3, \xi^{q}$ admits a block $P L$ structure, and

$$
f: D^{q+n+1} \longrightarrow T\left(\xi^{q}\right) \text { is f.h.t. on } S^{q+n},
$$


(1) if $n \neq 2$ there is well-defined element in $P_{n}$ (the $n$th surgery obstruction group) whose vanishing is necessary and sufficient for $f$ to be homotopic relative to $S^{q+n}$ to a f.h.t map.

(2) If $n=2$ the above conclusion holds if in addition $f^{-1}\left(M_{\xi}\right) \cap S^{q+n}$ is homotopy equivalent to a 2-manifold.

(3) If $h_{t}$ is a homotopy relative to $S^{q+n}$ from $f$ to $a$ f.h.t. map we may assume $h_{t}^{-1}\left(M_{\xi} \cup E\left(\xi^{q}\right) \times[0,1 / 2]\right)$ is constant. (Here $E\left(\xi^{q}\right) \times[0,1 / 2] \subset E\left(\xi^{q}\right) \times[0,1] /\left\{E\left(\xi^{q}\right) \times[1]\right\}=$ cone $\left(E\left(\xi^{q}\right)\right)$.)

We emphasize that we do not have a $P L$ structure for $\xi^{q}$, only the hypothesis that one exists.

Proof. We first need a lemma from the theory of $\mathrm{PD}$ spaces to the effect that 1-dimensional surgery is possible.

LEMMA. Let $M^{q+n} \stackrel{f}{\longrightarrow} T\left(\xi^{q}\right)$ with $3 \leqq n, 3 \leqq q, \pi_{1}(M)=0$ and $\pi_{1}(X)=0$. Assum $\xi$ also that $f$ is f.h.t. Then there is a homotopy $F: M \times I \rightarrow T\left(\xi^{q}\right), F \mid M \times[0]=f$ which is f.h.t. with $F^{-1}\left(M_{\xi}\right) \cap M \times[1]$ simply connected.

For a proof when $n \geqq 4$ see [10]. For $n=3$ use the fact [24] that any 3 dimensional $P D$ space is bordant to $S^{3}$. (In fact this bordism can be taken to be framed.)

We now return to a proof of the theorem. Put a block $P L$ structure on $\xi^{q}, E^{q} \rightarrow \xi^{q}$. In this structure shift $f$ slightly to $f^{\prime}$ which is block $P L$ transversal to a small block tube around the 0 -section of $E$. We clearly may do this so that $f^{-1}\left(M_{\xi}\right) \cap S^{q+n}$ and $f^{-1}\left(M_{\xi} \cup E(\xi) \times[0,1 / 2]\right)$ do not change. That is to shift a map to a $P L$ transversal one we need only alter it in a neighborhood of the 0 -section. Let $Y^{n+1}=f^{\prime-1}\left(0\right.$-section) and $Z=f^{-1}\left(M_{\xi}\right) . \quad Y^{n+1}$ is a $P L$ manifold of dimension $(n+1)$ with boundary equal to $Y \cap S^{q+n}=W^{n}$. $Z \cap S^{q+n}$ is an $n$-dimensional PD space, and $W^{n} \subset Z \cap S^{q+n}$. We claim that $W^{n} \subset Z \cap S^{q+n}$ naturally has the structure of a degree one normal map. This is proved by the following lemma.

LEMma 1.4. Let $f: M^{q+n} \rightarrow T\left(\xi^{q}\right)$ be f.h.t. with $f^{-1}\left(M_{\xi}\right)=X$. Suppose $\xi^{q}$ has a PL structure, $E$, and $h_{t}$ is a homotopy from $f$ to a block PL transversal map $f^{\prime}: M \rightarrow T\left(\xi^{q}\right)$ with the property that $h_{t}^{-1}\left(M_{\xi}\right)$ is constant. Let $f^{\prime-1}(0)=Y$.

Then $Y \subset X$ is a degree one map and is naturally covered by a bundle map. 


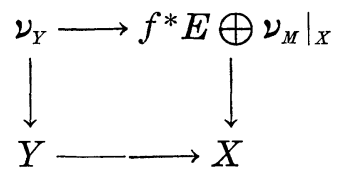

Proof. $Y \subset X$ since $Y=h_{1}^{-1}(0$-section $) \subset h_{1}^{-1}\left(M_{\xi}\right)=h_{0}^{-1}\left(M_{\xi}\right)=X$. $X=f^{-1}\left(M_{\xi}\right)$ is a codimension 0 -submanifold of $M^{q+n}$ and the fundamental class, $[X] \in H_{n}(X)$ is $f^{*} U_{\xi} \cap[X, \partial X]$ where $U_{\xi}$ is the Thom class of $\xi^{q}$. $i_{*}[Y] \in H_{n}(X)=h_{1}^{*} U_{\xi} \cap[X, \partial X]$. Since $h_{1} \cong f$ we see that $i_{*}[Y]=[X]$ in $H_{n}(X)$. Thus $Y \subset X$ is degree +1

$$
\begin{aligned}
\nu_{Y} & \left.\cong \nu_{Y \subset M} \bigoplus \nu_{M}\right|_{Y} \cdot \\
\nu_{Y \subset M} & =i^{*} h_{1}^{*} E \cong i^{*} h_{0}^{*} E=i^{*} f^{*} E .
\end{aligned}
$$

Thus we have a natural bundle map

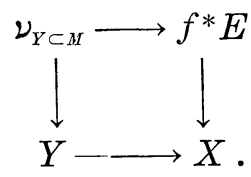

This induces

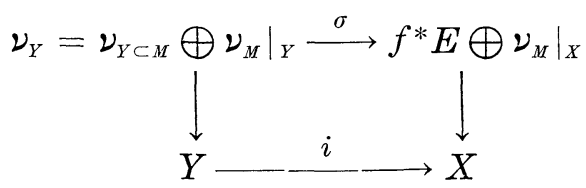

which is the bundle map covering $i$. This proves the lemma. Applying this to the situation in Theorem 1.3 we see that $W=Y \cap S^{q+n} \rightarrow$ $Z \cap S^{q+n}$ is a degree one normal map. Thus there is naturally associated an element in $P_{n^{\prime}}$ (the surgery obstruction of this normal map). We call this element $\sigma(f)$. We must show that:

(1) $\sigma(f)$ is well defined depending only on the homotopy class of $f$ (the homotopy required to be f.h.t. on $S^{q+n} \times I$ ) and

(2) if $\sigma(f)=0$, then we may put $f$ f.h.t. relative to $f \mid S^{q+n}$ keeping the preimage of $M_{\xi} \cup E(\xi) \times[0,1 / 2]$ constant.

We consider the second question first.

Case $n \geqq 5$. By the first lemma we may assume that

$$
\pi_{1}\left(Z \cap S^{q+n}\right)=0 .
$$

Thus if the surgery obstruction vanishes, we may do surgery until $W^{n} \rightarrow Z \cap S^{q+n}$ is a homotopy equivalence. These surgeries are realized by shifting $f^{\prime}$ slightly but may be done so as not to change the preimage of $M_{\xi}$ or $M_{\xi} \cup E\left(\xi^{q}\right) \times[0,1 / 2]$. Here we are using the fact 
that surgery is possible in codimension 3. Thus we may assume that in addition to all the above conditions that $W \subset Z \cap S^{q+n}$ is a homotopy equivalence. Thus $\left(Z \cap S^{q+n}\right) \cup($ tubular neighborhood of $Y$ ) is a PD space in $M$. It is the f.h.t. preimage of a map $f^{\prime}: D^{q+n+1} \rightarrow T\left(\xi^{q}\right)$. In constructing this map $f^{\prime}$ we have shifted $f$ on the boundary $S^{q+n}$, but we have not moved $f^{-1}\left(M_{\xi} \cap S^{q+n}\right)$ during the homotopy. Thus the standard collaring trick allows us to work relative to $S^{q+n}$. This verifies the second condition for $n \geqq 5$. For $n<5$ we use the same general argument but we must show that we can do the requisite surgery.

Case $n=4$. Here again we may assume that $Z \cap S^{q+n}$ is simply connected. Use the fact that in dimension 4 surgery is possible after sharping enough times with $S^{2} \times S^{2}$ see for example [24]. It is always possible to add to $Z \cap S^{q+4}$ a copy of $S^{2} \times S^{2}$ by performing a f.h.t. homotopy. Thus if the surgery obstruction vanishes we may make $W \subset Z \cap S^{q+4}$ a homotopy equivalence.

Case $n=3$. Here we may assume $Z \cap S^{q+3}$ is homotopy equivalent to $S^{3}$. Then we use the fact that any surgery problem with domain a $P L$ manifold and range $S^{3}$ may be solved.

Case $n=2$. Here we must use the extra hypothesis that $Z \cap S^{q+2}$ is homotopy equivalent to a 2-manifold, and the fact that for surgery problems between 2 manifolds surgery is possible (after a bordism of the range) if and only if the Kervaire obstruction vanishes.

Cases $n \leqq 1$ are trivial.

Using all these special tricks in low dimensions one then pushes the high dimensional argument through. The details are left to the reader.

We now turn to the question of the well-definedness of the obstruction $\sigma(f)$. We will use the following standard fact from surgery theory.

(*) If $f: L^{n+1} \rightarrow R^{n+1}$ is a normal map between PD space then $\sigma(f \mid \partial L)$ is 0 in $P_{n}$. See [2]. Suppose that $F: D^{q+n+1} \times I \rightarrow T\left(\xi^{q}\right)$ is f.h.t. when restricted to $S^{q+n} \times I$. Let $F^{-1}\left(M_{\xi}\right) \cap S^{q+n} \times I=X$ and $X \cap S^{q+n} \times[i]=X_{i}, i=0$ or 1 . Suppose that in $D^{q+n+1} \times[1]$ we have a PD pair $\left(R^{n+1}, \partial\right)$ obtained as follows. Let $M_{\xi}^{\prime} \subset M_{\xi}$ be a smaller copy of the mapping cylinder. We shift $F \mid D \times[1]$ to $G$ which is f.h.t. with respect to $M_{\xi}^{\prime}$ in such a way that $G^{-1}\left(M_{\xi}\right)=f^{-1}\left(M_{\xi}\right) \cap$ $D^{q+n+1} \times[1]$. Then $\partial R \subset X_{1}$ is a degree one normal map between PD spaces. (As an example, $R$ could be the tubular neighborhood of the preimage of the 0 -section under some map $P L$ transverse in 
a different $P L$ structure on $\xi^{q}$ ) Since the homotopy from $F$ to $G$ on $D \times[1]$ left the preimage of $M_{\xi}$ constant, we may assume that $F \mid D \times[1]=G$.

Now put a $P L$ structure on $\xi$ and shift $F$ slightly to $F^{\prime \prime}$ which is block $P L$ transverse. Do this keeping $F^{-1}\left(M_{\xi}\right)$ and $F^{-1}\left(M_{\xi}^{\prime}\right)$ constant.

Let $A=F^{\prime-1}$ (0-section), and $A_{i}=A \cap D \times[i]$. We wish to show that the surgery obstruction of $\partial A_{0} \rightarrow X_{0}$ equals that of $\partial R \rightarrow X_{1}$. This will then prove the well definedness of $\sigma(f)$. Since $A_{1} \rightarrow R$ is a degree one normal map, (*) implies that $\sigma\left(\partial A_{1} \rightarrow \partial R\right)$ is 0 . Also $\sigma\left(\partial A_{0} \rightarrow X_{0}\right)=\sigma\left(\partial A_{1} \rightarrow X_{1}\right)$ since $\partial A \rightarrow X$ is a degree one normal map. $\partial A_{1} \rightarrow X_{1}$ is the composite of two surgery problems $\partial A_{1} \stackrel{n_{1}}{\longrightarrow} \partial R \stackrel{n_{2}}{\longrightarrow} X_{1}$. Thus $\sigma\left(n_{2} \circ n_{1}\right)=\sigma\left(\partial A_{0} \rightarrow X_{0}\right)$ and $\sigma\left(n_{1}\right)=0$. If we knew that $\sigma\left(n_{2} \circ n_{1}\right)=$ $\sigma\left(n_{2}\right)+\sigma\left(n_{1}\right)$ it would follow that $\sigma\left(n_{2}\right)=\sigma\left(\partial A_{0} \rightarrow X\right)$ which is what we wish to prove.

In the signature case such an additivity formula is obvious. In the Kervaire invariant case it is not true_in general that $\sigma\left(n_{2} \circ n_{1}\right)=$ $\sigma\left(n_{2}\right)+\sigma\left(n_{1}\right)$. We need additional information.

In this case we may do surgery on $\partial A_{1} \stackrel{n_{1}}{\longrightarrow} \partial R$ until it is a homotopy equivalence. (In the low dimensions first cross with $C P^{2}$.) After doing this the crucial point is that the bundle map covering $\partial A_{1} \stackrel{n_{1}}{\longrightarrow} \partial R$ is $d n_{1}: \nu_{\partial A_{1}} \rightarrow \nu_{\partial R_{1}}$ or equivalently if we use the homotopy equivalence $n_{1}$ to identify $\partial A_{1}$ with $\partial R$ then the bundle maps given by $n_{2} \circ n_{1}$ and $n_{2}$ are the same. This is clear since $F^{\prime}$ is homotopic to $F$ as maps of spherical fiber spaces

$$
F^{\prime \prime}: \nu_{\partial R} \longrightarrow \nu_{Z} \text { and } F: \nu_{\partial_{A_{1}}} \longrightarrow \nu_{Z} \text {. }
$$

This proves the well definedness of $\sigma(f)$ and completes the proof of Theorem 1.3. Once we have this theorem the obstruction theory now follows by the usual formal type arguments. Setting it up is the aim of the rest of this section.

Statement of the obstruction theory. Let $\xi^{q} \rightarrow X$ be a spherical fiber space. Suppose we are give an open cover $\left\{U_{\alpha}\right\}$ of $X$ such that the base point of $X$,*, is in each $U_{\alpha}, U_{\alpha_{1}} \cap \cdots \cap U_{\alpha_{k}}$ is connected and simply connected for all $\left[\alpha_{1}, \cdots, \alpha_{k}\right]$, and such that $\xi \mid U \alpha$ admits a $P L$ structure. Form the open cover $\left\{V_{\alpha}\right\}$ of $T\left(\xi^{q}\right)$ where $V_{\alpha}=$ $M_{\xi} \mid U_{\alpha} \cup$ open cone $(E(\xi))$. Let $M$ be a triangulated $P L$ manifold, and suppose $f: M^{q+n} \rightarrow T\left(\xi^{q}\right)$ has the property that $f\left(\Delta^{i}\right)$ is contained in some $V_{\alpha}$ for each $\Delta^{i}$ in the triangulation of $M$, (i.e., $f$ is small with respect to the cover $\left.\left\{V_{\alpha}\right\}\right)$. Then there is an Eilenberg-MacLane obstruction theory for deforming $f$ inductively over the skeleta of $M$ until it is strongly f.h.t. such that if $f\left(\Delta^{i}\right) \subset V_{\alpha}$ then $\Delta^{i}$ stays in $V_{\alpha}$ during the deformation. 
NoTE. The conditions that all $U$ contain $*$ and that all intersections are connected and simply connected implies that $\pi_{1}(X)=0$ by Van Kampen's theorem. The converse is proved in next lemma.

LEMma 1.5. Any simply connected complex $X$ has a covering $\left\{U_{\alpha}\right\}$ satisfying all the above properties and such that for any spherical fibration $\xi^{q} \rightarrow X, \xi \mid U_{\alpha}$ admits a PL structure for each $U_{\alpha}$ in the cover.

Proof. Let $X$ be a simplicial complex. For each vertex $\alpha$ of $X$, let $U_{\alpha}$ be the open star in the second derived subdivision of the closed set $\left(X^{(2)} \cup\right.$ closed $\left.s t(\alpha)\right)$. Then any intersection $U_{\alpha_{1}} \cap \cdots \cap U_{\alpha_{n}}$ deforms to the two skeleton of $X$ which is connected and simply connected if $X$ is. Also $\xi \mid U_{\alpha}$ admits a $P L$ structure since any spherical fiber space over a 2 complex does.

DEFINITION. We call covers satisfying all the above properties good covers. Note that each simplex of $X$ is in one of the open sets of the cover. We will need this later in the paper.

Note. The cover that we constructed in the above lemma is natural with respect to simplicial maps. $F: X \rightarrow Y$. The condition that a map be small with respect to an open cover can always be realized by subdivison. However, it is not clear that if a map is strongly f.h.t. that the domain can be subdivided and the map shifted until it is strongly f.h.t. and small with respect to the cover. Philosophically, this smallness condition is related to the fact that $P L$ transversality can be accomplished by an arbitrarily small shift.

To set up the obstruction theory we need some corollaries of Theorem 1.3. Let $\pi_{q+n+1}\left(T\left(\xi^{q}\right)\right.$, s.f.h.t.) be, as a set, the set of homotopy classes of maps $D^{q+n+1} \stackrel{f}{\longrightarrow} T\left(\xi^{q}\right)$ such that $f$ is f.h.t. on $\partial D^{q+n+1}$ and in addition if $n=2$, then $f^{-1}\left(M_{\xi}\right)$ is homotopy equivalent to a 2manifold. The group structure is given by connected sum along the boundary where we sum small disks which go to the cone point.

Corollary 1.6. If $\xi^{q} \rightarrow X$ is a spherical fiber space with $X$ connected and simply connected and such that $\xi^{q}$ admits a PL structure then we have an isomorphism

$$
\pi_{q+n+1}\left(T\left(\xi^{q}\right), \text { s.f.h.t. }\right) \stackrel{\cong}{\longrightarrow} P_{n} \text { for } q \geqq 3 .
$$

Proof. Theorem 1.3 sets up a well defined function

$$
\pi_{q+n+1}\left(T\left(\xi^{q}\right) \text {, s.f.h.t. }\right) \stackrel{\tau}{\longrightarrow} P_{n}
$$


which is easily seen to be a group homomorphism. If $\tau(f)=0$, then $f$ is homotopic relative to $\partial D$ to a f.h.t. map. This, of course means $[f]=0 \in \pi_{q+n+1}\left(T\left(\xi^{q}\right)\right.$, s.f.h.t.). Thus $\tau$ is $1-1$. To show that $\tau$ is onto let $\alpha \in P_{n}$. Then there is a $G$-framed PD space

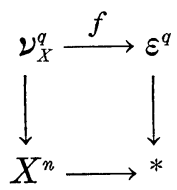

such that if we make $f P L$ transverse regular to $0 \in \varepsilon^{q}$, say $f^{-1}(0)=$ $Y^{n}$, then $\sigma\left(Y^{n} \rightarrow X^{n}\right)=\alpha$. Furthermore, $\phi: S^{q+n} \rightarrow T\left(\nu^{q}\right) \rightarrow S^{q}$ is homotopic to 0 . See [10]. Define $f: D^{q+n+1} T\left(\varepsilon^{q}\right)$ to be a homotopy from $\phi$ to *. One sees easily that $D^{q+n+1} \rightarrow T\left(\varepsilon^{q}\right) \subset T\left(\xi^{q}\right)$ is a map with obstruction $\alpha$. This proves $\tau$ is onto.

Definition of the obstruction cochain. Let $f: M^{q+n} \rightarrow T\left(\xi^{q}\right)$ be small with respect to the good cover as in 1.5, $\left\{V_{\alpha}\right\}$, and suppose $f \mid(q+i-1)$-skeleton is s.f.h.t. . Let $\Delta^{q+i}$ be a $(q+i)$-simplex in $M^{q+n}$. There is at least one of the open sets $U_{\rho}$ of $X$ for which $f \mid \Delta^{q+i}: \Delta^{q+i} \rightarrow T\left(\xi \mid U_{\rho}\right) \cup C(\xi)$ and $f \mid \partial \Delta$ is s.f.h.t. Since $U_{\rho}$ is connected and simply connected and $\xi \mid U_{\rho}$ admits a $P L$ structure, we assign to $\Delta^{q+i}, \sigma\left(f \mid \Delta^{q+i} \rightarrow T\left(\xi \mid U_{\rho}\right)\right)$. We must show that $\sigma$ is independent of $U_{\rho}$, i.e., if $f \mid \Delta^{q+i}$ is also contained in $U_{\rho}^{\prime}$, then $\sigma\left(f \mid \Delta^{q+i} \rightarrow T\left(\xi \mid U_{\rho^{\prime}}\right)\right)=$ $\sigma\left(f \mid \Delta^{q+i} \rightarrow T\left(\xi \mid U_{\rho}\right)\right)$. This follows easily from Lemma 1.4. This gives us a well defined cochain $o(f) \in \operatorname{Hom}\left(C_{q+i}(M), P_{\imath-1}\right)$.

$o(f)$ is a cocycle. To see this we must show that $\left\langle o(f), \partial \Delta^{q+\imath+1}\right\rangle=0$. We have $f: \Delta^{q+i+1} \rightarrow T\left(\xi \mid U_{\rho}\right)$ and $\xi \mid U_{\rho}$ admits a $P L$ structure. In $\partial \Delta^{q+i+1}=S^{q+i}$ take a collar neighborhood of the $(q+i-1)$-skeleton, $C$. We may easily shift $f$ to be f.h.t. on this neighborhood. $\partial C$ has one component equal to $S^{q+i-1}$ for each $(g+i)$-simplex of $\partial \Delta_{q+i+1}$.

Shift all of $C P L$ transverse to some $P L$ structure on $\xi \mid U_{\rho}$. Let $Y=$ the preimage of the 0 -section then $Y \subset f^{-1}\left(M_{\xi}\right) \cap C$ is a degree one normal map. Thus $\sigma\left(Y \cap \partial C \subset f^{-1}\left(M_{\xi}\right) \cap \partial C\right)=0$. On the other hand one sees that this latter surgery obstruction is $\sum_{j=0}^{q+2-1}\left(-1_{j}\right) o(j$ th face). Where $o\left(j\right.$ th face) means $\left\langle o(f), j\right.$ th face of $\left.\Delta^{q+i+1}\right\rangle$. Thus $\langle o(f)$, $\left.\partial \Delta^{q+\imath+1}\right\rangle=0$.

If $o(f)=0$ as a cochain we may shift $f$ relative to the $(q+i-1)$ skeleton of $M$ to $f_{1}$ which is f.h.t. on the $(q+i)$ skeleton. Furthermore, we can do this in such a way as to keep $\Delta^{j}$ in V $\rho$ whenever $f\left(\Delta^{j}\right) \subset V \rho$.

Proof. We work one $(q+i)$-simplex at a time. Since $f\left(\Delta^{q+i}\right) \subset V_{\rho}$. 
We have all the hypothesis to apply Theorem 1.3. The result follows easily from it. Note that we may do this keeping the preimage of $M_{\xi} \cup E \times[0,1 / 2]$ constant by Property 3 of 1.3 .

By shifting $f$ on the $(q+i-1)$ skeleton by a homotopy (but not a transversal homotopy) relative to the $(q+i-2)$-skeleton we may change o(f) by an arbitrary coboundary.

Proof. Here we will use the fact that the base point is in all of the $U_{\rho}$ 's, and thus $T\left(\varepsilon^{q}\right) \subset V_{\rho}$ for every $\rho$. We will change $o(f)$ by $\delta e_{i-1}$ where $\left\langle e_{i-1}, \Delta_{0}^{q+i-1}\right\rangle=\alpha \in P_{i-1}$ and $\left\langle e_{i-1}, \Delta^{q+i-1}\right\rangle=0$ for all other $\Delta^{q+i-1}$. Given $\alpha \in P_{i-1}$ we know there is a map $S^{q+i-1} \stackrel{\bar{\alpha}}{\longrightarrow} T\left(\varepsilon^{q}\right)$ which is homotopic to 0 , and which is transversal on $S^{q+i-1}$ obstruction $\alpha$ to extending the transversality on $D^{q+i}$. Pick a disk a disk $D_{0}^{q+i-1} \subset \Delta_{0}^{q+i-1}$ missing $f^{-1}\left(M_{\xi}\right)$ and change $f$ by a homotopy on this disk relative to its boundary until on the interior of $D_{0}^{q+\imath-1}$ it agrees with $\bar{\alpha}$. Then use homotopy extension to extend this to all of $M^{q+n}$. One checks easily that this changes $o(f)$ exactly by $\delta e_{i-1}$. This completes the proof of the obstruction theory.

Note. There is one condition that we must verify and that is in going from the $(q+1)$-skeleton to the $(q+2)$-skeleton that all $\left(f^{-1}(M) \cap \Delta^{q+2}, f^{-1}\left(M_{\xi} \cap \partial \Delta^{q+2}\right)\right.$ are homotopy equivalent to $P L 2$-manifolds with boundary. This, howevery, is easy to arrange. Then for any $\Delta^{q+3}, f^{-1}\left(M_{\xi}\right) \cap \partial^{q+3}$ will be homotopy equivalent to a 2-manifold.

Note. If we wish to change $o(f)$ by $\delta e_{i-1}$ where $\left\langle e_{i-1}, \Delta_{0}^{q+i-1}\right\rangle=1$ and $\left\langle e_{i-1}, \Delta^{q+i-1}\right\rangle=0$ for all other $\Delta^{q+i-1}$ we may do this keeping $f^{-1}\left(M \cup_{\xi} E \times[0,1 / 2]\right)$ constant providing only that

$$
f^{-1}\left(M_{\xi} \cup E \times\left[0, \frac{1}{2}\right]\right) \cap \Delta_{0}^{q+i-1}
$$

contains a small disk $D^{q+i-1}$. To see this we use the following lemma

LeMma 1.8. Given $\alpha \in P_{n}$, there is a map $g: D^{q+n+1} \rightarrow T\left(\xi^{q}\right)$, which is f.h.t. on $S^{q+n}$ with obstruction $\alpha$ with $g\left(D^{q+n+1}\right)$ contained in an arbitrarily small neighborhood of $* \in T\left(\xi^{q}\right)=S^{q}$.

Proof. The argument in 1.6 produces $g: D^{q+n+1} \rightarrow T\left(\varepsilon^{q}\right)$ f.h.t. on $S^{q+n}$ with obstruction $\alpha$. Let 0 be the image of the base of $\varepsilon^{q}$ in $T\left(\varepsilon^{q}\right)$ and 1 the antipodal point on $T\left(\varepsilon^{q}\right)=S^{q}$. Leaving $g / S^{q+n}$ fixed near 0 we wish to make it miss 1 . Since we have $g: S^{q+n} \rightarrow S^{q}$ the only obstruction to making $g$ miss the point $1 \in S^{q}$ is the element in 
framed bordism $g^{-1}(1)$ after $g$ has been shifted transverse to 1 . But since $g$ bounds $D^{q+n+1}$ this element is 0 . Thus we make shift $g$ slightly near $1 \in S^{q}$ to make it miss $1 \in S^{q}$. Once we have $g \mid \partial D^{q+n+1}$ missing 1 by changing the map by an element in $\pi_{q+n+1}\left(S^{q}\right)$ we may make $g$ on all of $D^{q+n+1}$ miss 1 .

Now if we wish to change $o(f)$ by $\delta e_{i-1}$ as above and if $\Delta_{0}^{q+i-1} \cap$ $f^{-1}\left(M_{\xi} \cup E \times[0,1 / 2]\right)$ contains a disk $D_{0}^{q+i-1}$ we may first assume that this disk is contained in $f^{-1} E \times[0,1 / 2]$ and that it maps by $f$ to a point. Now use the previous lemma to shift $f$ near this disk. Since $g\left(D^{q+n+1}\right) \subset M_{\xi} q \subset T\left(E^{q}\right)$ the process never moves any point near $D_{0}^{q+i-1}$ outside of $M_{\xi} \cup E \times[0,1 / 2]$.

Note. Since this theory is "one simplex at a time relative to its boundary" there is clearly a relative obstruction theory for deforming a map $f: M^{q+n} \rightarrow T\left(\xi^{q}\right)$ relative to a subcomplex $K$ on which it is already strongly f.h.t. The obstructions lie in $H^{q+i}\left(M, K ; P_{i-1}\right)$.

Also note that if $L$ is a subcomplex of $M$ which $f$ sends to the cone point, then, after $f$ is shifted to $f^{\prime}$ which is strongly f.h.t., $f^{\prime}$ maps $L$ into $T(\xi \mid U)$ where $U$ is an arbitrary contractable open set containing the base point.

NoTE. The obstruction theory is natural with respect to simplicial maps of bases covered by bundle maps, i.e.,

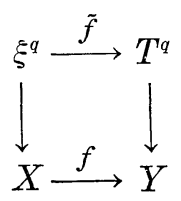

where $f$ is simplicial. The fact that $f$ is simplicial implies that it respects the chosen covers on $Y$ and $X$. From this naturality follows easily. The obstruction theory is also natural with respect to suspensions.

Namely given $f: M^{q+n} \rightarrow T\left(\xi^{q}\right)$ small with respect to the cover we may suspend to form $M \times I \stackrel{\sum f}{\longrightarrow} T\left(\xi^{q} \oplus e^{1}\right)$ where $\sum f \mid M \times \stackrel{\circ}{I}$ is the point map. The obstructions for $f$ lie in $H^{q+*}\left(M^{q+n} ; P_{*}\right)$ and the obstructions for $\sum f$ are in $H^{q+1+*}\left(M^{q+n} \times I, M^{q+n} \stackrel{\circ}{I} ; P_{*}\right) \cong H^{q+1+*}\left(\sum M ; P_{*}\right)$. If we have a deformation of $f$ to be f.h.t. on the $(q+i)$-skeleton, then this gives a deformation of $\sum f$ on the $(q+i+1)$-skeleton of $M \times I$. The obstructions to extending the maps further agree under the suspension isomorphism.

2. First consequences of the obstruction theory. In this section we wish to formulate homotopy theoretic consequences of the obstruc- 
tion theory of $\S 1$. Let $\xi^{q} \rightarrow X$ be a spherical fiber space, $q \geqq 3, \pi_{1}(X)=0$ and $\left\{U_{\rho}\right\}$ be the cover of $X$ and $\left\{V_{\rho}\right\}$ the associated cover of $T\left(\xi^{q}\right)$ as in 1.5. (We shall always work with these covers unless otherwise specified.)

DEFINITION 2.1. $W_{\xi^{q}}$ is the semi-simiplicial complex of all strongly f.h.t. maps $\Delta^{i} \rightarrow T\left(\xi^{q}\right)$ which are small with respect to the cover $\left\{V_{\rho}\right\}$. Denote by $\widetilde{T}\left(\xi^{q}\right)$ the total singular complex of $T\left(\xi^{q}\right)$. Then we have a natural map $W_{\xi^{q}} \rightarrow \widetilde{T}\left(\xi^{q}\right)$. Let $F_{\xi^{q}}$ be the homotopy theoretic fiber of this inclusion. The following is a representative semi-simplicial complex for $F$. The $i$-simplices are maps $h: \Delta^{i} \times I \rightarrow T\left(\xi^{q}\right)$ with $h \mid \Delta^{i} \times\{0\}$ strongly f.h.t. and small with respect to the cover and $h \mid \Delta^{i} \times\{1\}: \Delta^{i} \times\{1\} \rightarrow$ cone point.

We shall construct in this section homomorphisms from bordism groups of this fiber to the surgery obstruction groups These homomorphism will measure the obstruction to global transversality. We shall show that they satisfy all of the natural compatibility relations and product formulae analogous to those for surgery obstructions. (In fact the homomorphisms are surgery obstructions.) Later in this paper we shall use these homomorphisms and the product formulae that they satisfy to derive further homotopy theoretic consequences. This is the reason that we state them now. First, however, we relate the homotopy groups of $F_{\xi}$ to the surgery obstruction group.

THEOREM 2.2. $\pi_{i}\left(F_{\xi^{q}}\right) \cong P_{i-q}$ provided that $q \geqq 3$.

Proof. We show that there is a natural $\pi_{i}\left(F_{\xi^{q}}\right) \cong \pi_{i+1}\left(T\left(\xi^{q}\right)\right.$, strongly f.h.t.). But by the obstruction theory $\pi_{i+1}\left(T\left(\overrightarrow{\xi^{q}}\right)\right.$, strongly f.h.t. $) \cong P_{i-q}$. An element in $\pi_{i}\left(F_{\xi^{q}}\right)$ is a map $S^{i} \times I \stackrel{h}{\longrightarrow} T\left(\xi^{q}\right)$ such that $h \mid \vec{S}^{i} \times\{0\}$ strongly f.h.t. and $S^{i} \times\{0\}$ is triangulated so that $h$ is small with respect to the cover and $h\left(S^{i} \times\{1\}\right)=$ cone point. Thus we may factor $h$ through $S^{i} \times I / S^{i} \times\{1\}=D^{i+1}$. This gives the map $\pi_{i}\left(F_{\xi^{q}}\right) \rightarrow \pi_{i+1}\left(T\left(\xi^{q}\right)\right.$, strongly f.h.t.). The proof that it is well defined and the construction of the the inverse are analogous.

LEMMA 2.3. The PL bordism groups of the geometric realization of $F_{\xi} q$ are naturally isomorphic to the following groups. As representative elements we take $H: M^{n} \times I \rightarrow T\left(\xi^{q}\right)$ with $H \mid M \times\{0\}$ small with respect to the cover and strongly f.h.t. and $H \mid M \times 1: M \times 1 \rightarrow$ cone point. Such an element is equivalent to zero if there is an $\bar{H}: W^{n+1} \times I \rightarrow T\left(\xi^{q}\right)$ with $\bar{H} \mid W \times\{0\}$ small with respect to the cover and strongly f.h.t. and $\bar{H} \mid W \times\{1\} \rightarrow$ cone point. And $\partial W=M$ with 
$\bar{H} \mid \partial W=H$.

Proof. This follows from the standard arguments in semi-simplicial theory.

THEOREM 2.4. There are natural maps

$$
\begin{aligned}
& \Omega_{q+i}^{P_{L}} \quad\left(F_{\xi^{q}}\right) \stackrel{\odot}{\longrightarrow} P_{i} \\
& \Omega_{q+i}^{P L} \quad\left(F_{\xi q} ; \boldsymbol{Z} / n\right) \stackrel{\mathcal{O}_{n}}{\longrightarrow} P_{i} \otimes Z / n \\
& \eta_{q+2 i}^{P L} \quad\left(F_{\xi q}\right) \stackrel{O}{\longrightarrow} Z / 2 \text { satisfying }
\end{aligned}
$$

(i) $O$ and the $\left[\mathcal{O}_{n}\right]$ are compatible with the natural maps $Z \stackrel{\text { red }}{\longrightarrow} Z / n$ and $Z / n \stackrel{\text { inc }}{\longrightarrow} Z / n \cdot k$ in dimensions $q+4 *+1$.

(ii) $\mathscr{O}_{1} \mathscr{O}_{2}$ and $\tilde{\mathcal{O}^{2}}$ are compatible with the natural maps

$$
\Omega_{q+4 *-1}\left(F_{\xi^{q}}\right) \longrightarrow \Omega_{q+4 *-1}\left(F_{\xi^{q}} ; Z / 2\right) \longrightarrow \eta_{q+4 *-1}\left(F_{\xi^{q}}\right) \text {. }
$$

(iii) These maps measure the obstruction to global transversality.

(iv) They satisfy the product formulae as in [15] and [22]

(a) $\eta_{*}\left(F_{\xi^{q}}\right) \otimes \eta_{*}(p t) \rightarrow \eta_{*}(F) \rightarrow P_{*-q} \quad$ sends $\quad(M, f) \otimes N \quad$ to $\mathcal{O}(M, f) \cdot \chi(N)$

(b) $\Omega_{*}^{P L}\left(F_{\xi}\right) \otimes \Omega_{*}^{P L}(p t) \rightarrow \Omega_{*}^{P L}\left(F_{\xi}\right) \rightarrow P_{*-q} \quad$ sends $\quad(M, f) \otimes N^{n} \quad$ to $\mathscr{O}(M, f) \cdot I(N)$

(c) $\Omega_{*}^{P L}(F ; Z / n) \otimes \Omega_{*}^{P L}((p t) ; z / n) \rightarrow \Omega_{*}^{P L}(F ; Z / n) \rightarrow P_{*} \otimes Z / n$ for $n$ odd sends $(M, f) \otimes N$ to $\mathcal{O}_{n}(M, f) \cdot I(N)$ for $n=2^{k}$ the map sends

$$
\left(M^{m}, f\right) \otimes N^{l} \text { to }\left\{\begin{array}{cr}
\mathcal{O}(M, f) \cdot I(N) & l \equiv 0(4) \\
2^{k-1} \mathcal{O}_{n}(M, f) \cdot d\left(\delta N^{l}\right) & m, l \equiv 2(4) \\
2^{k-1} \mathcal{O}(f \mid \delta M) \cdot d\left(N^{l}\right) & m \equiv 3(4) \\
& l \equiv 1(4) \\
0 & \text { otherwise }
\end{array}\right\} .
$$

Here $d\left(L^{4 k-1}\right)$ is the de Rham invariant of skew symmetric linking pairing on a group associated with the homology of $L$. It is given by the characteristic number $\left\langle V S q^{1} V,[L]\right\rangle \in Z / 2$. See [15]. If $M$ is a $Z / 2^{k}$-manifold, $\delta M$ means the closed codimension one manifold which is the singularity set.

Proof. Construction of the homomorphisms. Let $M^{q+i} \times I \stackrel{H}{\longrightarrow}$ $T\left(\xi^{q}\right)$ with $H \mid M \times\{0\}$ strongly f.h.t. and small with respect to $\left\{V_{\rho}\right\}$, and $H \mid M \times\{1\}$ the point map to the cone point. Triangulate $M \times I$ relative to $M \times\{0\}$ so that $H$ is small with repect to $\left\{V_{\rho}\right\}$. Since $H^{*}\left(M \times I, M \times\{0\} ; P_{*-q-1}\right)=0$ we may shift $H$ relative to $M \times\{0\}$ 
to $H^{\prime}$ which is strongly f.h.t. Since $H(M \times\{1\})=$ cone point, $H^{\prime}(M \times\{1\}) \subset T(\xi \mid U) \cup$ cone $E(\xi)$ where $U$ is a contractible neighborhood of the base point. Thus $H^{\prime-1}\left(M_{\xi}\right) \subset M \times\{1\}$ is a $\mathrm{PD}$ space with a $G$-framed normal bunle in $M \times\{1\}$. We also have a homotopy of $M \times\{1\} \rightarrow T\left(\nu_{H^{-1}(M \xi) \cap M \times\{1\}}\right) \rightarrow T(\xi \mid U)$ to zero. From this we shall read off the obstruction $o$. Let $X^{n}=H^{-1}\left(M_{\xi}\right) \cap M \times\{1\}$ and $t: \nu_{X \subset M \times[1\}} \rightarrow \varepsilon^{n}$ be the trivialization. Put $t P L$ transversal to $0 \in \varepsilon^{n}$. This gives $Y^{n} \stackrel{f}{\longrightarrow} X^{n}$ a degree one normal map. Define $O(M \times I, H)$ to be the surgery obstruction of this normal map. If $M$ is closed and oriented, then it lies in $P_{n}$. If $M$ is unoriented, then it lies in 0 if $n$ is odd and $\boldsymbol{Z} / 2$ if $n$ is even. If $M$ is a closed oriented $Z / k$-manifold, then it lies in $P_{n} \otimes Z / k$. (See [15] $\S 1$ and 5.)

We now prove that $O$ is well defined depending only on the cobordism class of $(M \times I, H)$. Let $J: W^{q+n+1} \times I \rightarrow T\left(\xi^{q}\right)$ satisfy

(1) $J \mid W \times\{0\}$ is small with respect to $\left\{V_{\rho}\right\}$ and s.f.h.t.

(2) $J \mid W \times\{1\}: W \times\{1\} \rightarrow$ cone point

(3) $\partial W=M$ and $J \mid \partial W \times I=H$. (i.e., $(W, J)$ is a bordism of $(H, H)$ to 0 in $\left.F \xi_{q}\right)$

Since $H^{*}\left(W \times I, W \times\{0\}, P_{*-n-1}=0\right.$, we may deform $J$ until it is s.f.h.t. on all of $W \times I$, relative to $W \times\{0\}$. This then gives a $G$-framed PD subspace of $W \times\{1\},\left(Y^{n+1}, t: \nu_{Y \subset W \times\{1\}} \rightarrow \varepsilon^{n}\right)$. The surgery obstruction of $t^{-1}(0) \cap \nu_{\partial Y} \rightarrow \partial Y^{n}$ is then 0 . Thus to show bordism invariance of $\mathcal{O}$, we need only show that this construction on $(M \times I, H)$ gives the same obstruction as any other similar construction.

To show this, suppose we have $H: M \times I \rightarrow T\left(\xi^{q}\right)$ as above and two deformations of $H$ to $H^{\prime}$ and $H^{\prime \prime}$ both relative to $M \times\{0\}$ and both s.f.h.t. on all of $M \times I$. Let $\bar{H}: M^{q+n} \times I \times \rightarrow T\left(\xi^{q}\right)$ be a homotopy between $H^{\prime}$ and $H^{\prime \prime}$ with $\bar{H} \mid M \times\{0\} \times I=(H \mid M \times\{0\}) \times I$ and $\bar{H} \mid M \times\{1\} \times I$ contained in $T(\xi \mid U) \cup$ cone $E(\xi)$ where $U$ is a contractible neighborhood of $*$ in $X$. Then $H \mid M \times[I \times \dot{I} \cup\{0\} \times I]$ is s.f.h.t. Since $H^{*}(M \times I \times I, M \times[I \times \dot{I} \cup\{0\} \times I])$ is 0 we may shift $\bar{H}$ to s.f.h.t. $\quad \bar{H}^{-1}\left(M_{\xi}\right) \cap M \times\{1\} \times I$ then is a $G$-framed PD bordism between $H^{\prime-1}\left(M_{\xi}\right) \cap M \times\{1\}$ and $H^{\prime \prime-1}\left(M_{\xi}\right) \cap M \times\{1\}$. This shows the two surgery obstructions agree, and proves $\mathcal{O}$ is well defined.

We now check that these homomorphisms satisfy (i)-(iv) as claimed in the theorem.

(i) and (ii). The compatibilities claimed in (i) and (ii) follow easily from the analogous compatibilities for surgery obstructions, see [15] $\S 55$ and 6 .

(iii) Global transversality. We say that $f: M^{q+n} \rightarrow T\left(\xi^{q}\right)$ is 
globally transversal if it is f.h.t. (but not necessarily f.h.t. on each simplex). If $f$ is globally transversal, then $f^{-1}\left(M_{\xi}\right)$ is a $\mathrm{PD}$ space of formal dimension $n$. We will show here that if $\mathcal{O}(M \times I, H)=0$, then we may deform $H$ relative to $M \times \dot{I}$ to make it globally transversal and conversely if $H$ may be deformed relative to $M \times \dot{I}$ until it is globally transversal then $\mathscr{O}(M \times I, H)=0$.

If $\mathscr{O}(M \times I, H)=0$, we deform $H$ by a f.h.t. map to make $H^{-1}\left(M_{\xi}\right) \cap M \times\{0\}$ connected and simply connected. Let $J$ be the homotopy from the cone point to $H^{\prime} \mid M \times\{1\}$. The image of $J$ is contained in $T\left(\xi^{q} \mid U\right)$ where $U$ is a contractible neighborhood of the base point in $X$, and $J$ of one end goes to the cone point. Thus we may shift $J$ to be $P L$ transverse relative to this end: Let $Y^{n}$ be the preimage.

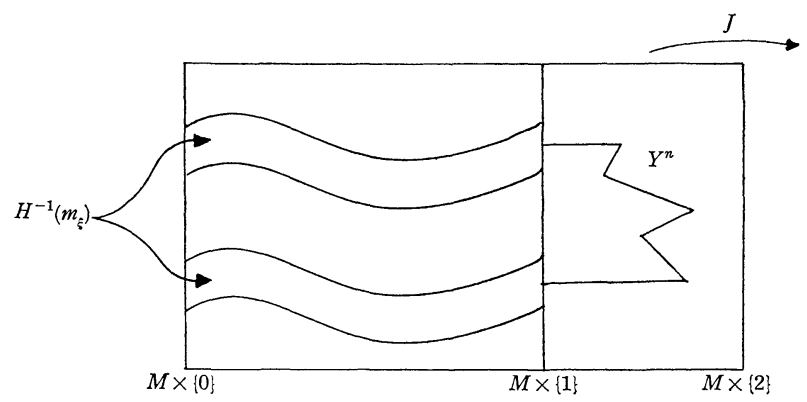

The element $\mathscr{O}(M \times I, H)$ then is by definition the surgery obstruction of $Y \cap M \times\{1\} \subset H^{\prime-1}\left(M_{\xi}\right) \cap M \times\{1\}$. If it vanishes then we may do surgery by further shifts of $J$ until $Y \cap M \times\{1\} \subset H^{\prime-1}\left(M_{\xi}\right) \cap M \times\{1\}$ is a homotopy equivalence. Then $H^{\prime} \cup J$ will be f.h.t. and homotopic relative to the ends to $H$.

REMARK 2.5. Note that $\mathscr{O}(M \times I, H)$ is the surgery obstruction of a surgery problem where the domain bounds and the range is PD cobordant to $H^{-1}\left(\mathscr{L}_{\xi}\right) \cap M \times\{0\}$. Note also that if $\operatorname{dim}(M)=$ $q+i$ with $i$ odd, then the obstruction to transversality lives on the Bockstein of $M$. That is, the obstruction to global transversality is $\mathcal{O}\left(\delta M \times I,\left.H\right|_{\delta M \times I}\right) \in P_{i-1}$.

Now suppose that $H: M \times I \rightarrow T\left(\xi^{q}\right)$ is homotopic rel $M \times \dot{I}$ to a globally transversal map. We wish to show that $\mathscr{O}(M \times I, H)=0$. This will require a brief digression and alternative definition of $\mathcal{O}(M \times I, H)$.

Roughly, we will deform $H: M \times I \rightarrow T\left(\xi^{q}\right)$ rel $M \times\{1\}$ (rather than $M \times\{0\})$ to a globally transversal map $H^{\prime}$ with respect to a smaller mapping cylinder $\mathscr{A}_{\xi^{\prime}} \subset \mathscr{A}_{\xi^{*}}$. We will do this in such a way that $H^{-1}\left(\mathscr{C l}_{\xi}\right)$ remains setwise fixed. It then follows from a slight 
generalization of Lemma 1.4 that the inclusion $\left(H^{\prime}\right)^{-1}\left(\mathscr{M}_{\xi^{\prime}}\right) \cap M \times\{0\} \rightarrow$ $H^{-1}\left(\mathscr{C l}_{\xi}\right) \cap M \times\{0\}$ is a degree one noamal map of PD spaces.

There are theories of surgery on PD spaces ([8], [18]), but for our applications, it is enough to observe that given a degree one normal map $X \rightarrow Y$ of $i$-dimensional $Z / n$-PD spaces, there is defined homotopy theoretically an obstruction $s(X \rightarrow Y) \in P_{i} \otimes Z / n$ ([3], [4]). $s(X \rightarrow Y)$ an invariant of the normal bordism class of $X \rightarrow Y$ and vanishes if $X \rightarrow Y$ is a homotopy equivalence. If $X$ is a $P L$-manifold, $s(X \rightarrow Y)$ is the usual surgery obstruction.

We will argue that in the situation above, $\mathscr{O}(M \times I, H)=$ $s\left(\left(H^{\prime}\right)^{-1}\left(\mathscr{C}_{\xi^{\prime}}\right) \cap M \times\{0\} \rightarrow H^{-1}\left(\mathscr{C}_{\xi}\right) \cap M \times\{0\}\right)$. It follows that if $H$ is globally transversal, then $\mathscr{O}(M \times I, H)=0$, since then $\left(H^{\prime}\right)^{-1}\left(\mathscr{A}_{\xi^{\prime}}\right) \rightarrow$ $H^{-1}\left(\mathscr{C}_{\xi}\right)$ is a normal map of $\mathrm{PD}$ spaces with boundary equal to $\left(H^{\prime}\right)^{-1}\left(\mathscr{C l}_{\xi^{\prime}}\right) \cap M \times\{0\} \rightarrow H^{-1}\left(\mathscr{C l}_{\xi}\right) \cap M \times\{0\}$.

Now return to $H: M \times I \rightarrow T\left(\xi^{q}\right)$. Let $\mathscr{C}_{\xi^{\prime}} \subset \mathscr{C l}_{\xi}$ be a slightly smaller mapping cylinder for $\xi$. By Theorem 1.3.13 and Note 1.7, the obstruction theory allows us to deform $H$ rel $M \times\{1\}$ to a globally transversal map $H^{\prime}$ with respect to $\mathscr{C}_{\xi^{\prime}}$, such that $H^{-1}\left(\mathscr{C}_{\xi}\right)$ is kept setwise fixed throughout the deformation, provided that $H^{-1}\left(\mathscr{C}_{\xi}\right) \cap \Delta^{a+j}$ contains a disc $D^{j}$ for each cell $\Delta^{q+j}$ of $M \times I-M \times\{1\}, j \geqq 1$. So our first step is to run thickened arcs from $H^{-1}\left(\mathscr{C}_{\xi}\right)$ to any such cell $d^{q+j}$ which does not intersect $H^{-1}\left(\mathscr{C}_{\xi}\right)$. This can be interpreted as a deformation of $H$ with does not change homotopy properties of the inclusion $H^{-1}(\xi) \subset H^{-1}\left(\mathscr{C} \mathscr{C}_{\xi}\right)$. In particular, $H^{-1}\left(\mathscr{C l}_{\xi}\right) \cap M \times\{0\}$ remains a $\mathrm{PD}$ space.

This step provides us with our normal map of $\mathrm{PD}$ spaces $\left(H^{\prime}\right)^{-1}\left(\mathscr{C l}_{\xi^{\prime}}\right) \cap M \times\{0\} \rightarrow H^{-1}\left(\mathscr{C}_{\xi}\right) \cap M \times\{0\}$. It is not difficult to use the obstruction theory in a similar manner to prove that the normal bordism class of this map is an invariant of the bordism class of the map $M \rightarrow F_{\xi_{q}}$ which corresponds to $H: M \times I \rightarrow T\left(\xi_{q}\right)$. Thus $\mathcal{O}^{\prime}(M \times I, H)=s\left(\left(H^{\prime}\right)^{-1}\left(\mathscr{C l}_{\xi^{\prime}}\right) \cap M \times\{0\} \rightarrow H^{-1}\left(\mathscr{C l}_{\xi}\right) \cap M \times\{0\}\right)$ is an invariant of the bordism class of $M \rightarrow F_{\xi^{q}}$. To complete the proof of Theorem 2.4(iii), we need to prove that $\mathcal{O}^{\prime}(M \times I, H)=$ $\mathcal{O}(M \times I, H)$.

Recall that $\mathcal{O}(M \times I, H)$ is defined as follows. Deform $H$ rel $M \times\{0\}$ to a transversal map $H^{\prime \prime}: M \times[0,1] \rightarrow T\left(\xi^{q}\right)$ such that $H^{\prime \prime}(M \times\{1\}) \subset$ $T\left(\left.\xi^{q}\right|_{U}\right)$, where $U$ is a contractible neighborhood of the basepoint of $B \xi^{q}$. Let $J: M \times[1,2] \rightarrow T\left(\left.\xi^{q}\right|_{/}\right)$be the homotopy, given by the deformation of $H$, from $\left.\left.J\right|_{M \times\{1\}} \equiv H^{\prime \prime}\right|_{M \times\{1\}}$ to $\left.J\right|_{M \times\{2\}} \equiv \infty, \infty \in T\left(\xi^{q}\right)$ the cone point. Using a $P L$-structure on $\left.\xi^{q}\right|_{U}$, we may assume that $J$ is $P L$-transversal to a smaller mapping cylinder $\mathscr{A}_{\xi^{\prime}} \subset \mathscr{M}_{\xi^{\prime}}$. Then $Y^{n}=J^{-1}\left(B \xi^{q}\right)$ is a $P L$-manifold, and $\partial Y^{n}=J^{-1}\left(B \xi^{q}\right) \cap M \times\{1\} \rightarrow$ $J^{-1}\left(\mathscr{C}_{\xi}\right) \cap M \times\{1\}=\left(H^{\prime \prime}\right)^{-1}\left(\mathscr{C}_{\xi}\right) \cap M \times\{1\}$ is a degree one normal map. By definition, $\mathcal{O}(M \times I, H)=s\left(\partial Y^{n} \rightarrow\left(H^{\prime \prime}\right)^{-1}\left(\mathscr{C l}_{\xi}\right) \cap M \times\{1\}\right)$. 


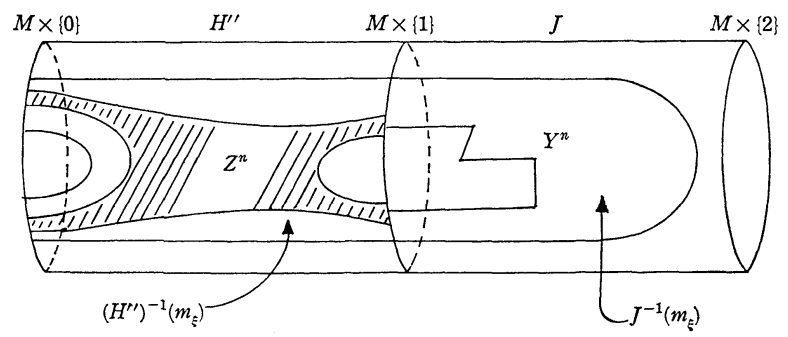

Now, $\left.H^{\prime \prime}\right|_{M \times\{1\}}$ is transversal with respect to both $\mathscr{C}_{\xi}$ and $\mathscr{C}_{\xi^{\prime}}$. We may as well assume, just as above, that $\left(H^{\prime \prime}\right)^{-1}\left(\mathscr{C L}_{\xi}\right)$ meets all cells of $M \times\{0,1\}-M \times\{1\}$. Then we can deform $H^{\prime \prime}$ rel $M \times\{1\}$ to $H^{\prime \prime \prime}$, which is transversal with respect to $\mathscr{C}_{\xi^{\prime}}$ on all of $M \times[0,1]$, keeping $\left(H^{\prime \prime}\right)^{-1}\left(\mathscr{C l}_{\xi}\right)$ setwise fixed. This gives a PD space with boundary, $Z^{n}=\left(H^{\prime \prime \prime}\right)^{-1}\left(\mathscr{C}_{\xi^{\prime}}\right) \subset\left(H^{\prime \prime}\right)^{-1}\left(\mathscr{C l}_{\xi}\right)$, with $Z^{n} \cap M \times\{1\}=\partial Y^{n}$. We now compute

$$
\begin{aligned}
\mathcal{O}(M \times I, H) & =s\left(\partial Y^{n} \longrightarrow\left(H^{\prime \prime}\right)^{-1}(\mathscr{C l}) \cap M \times\{1\}\right) \\
& =s\left(Z^{n} \cap M \times\{0\} \longrightarrow\left(H^{\prime \prime}\right)^{-1}\left(\mathscr{C l}_{\xi}\right) \cap M \times\{0\}\right) \\
& =\mathcal{O}^{\prime}\left(M \times I, H^{\prime \prime} \cup J\right) \\
& =\mathcal{O}^{\prime}(M \times I, H)
\end{aligned}
$$

as desired.

Theorem 2.4(iii) is one of the key points of the paper. It is precisely here that we obtain a global obstruction to transversality (in a rather specific situation), using a call-by-cell obstruction theory. The remainder of the paper exploits this global obstruction.

(iv) Product formulae. Suppose we have $H: M \times I \rightarrow T\left(\xi^{q}\right)$ as above, and we deform $H$ relative to $M \times\{0\}$ to $H^{\prime}$ which is s.f.h.t. Let $\left(Y^{n} \subset M \times\{1\}, t: \nu_{Y \subset M \times\{1\}} \rightarrow \varepsilon^{q}\right.$ ) be the resulting $G$-framed PD subspace in $M \times\{1\}$. If we cross $(M \times I, H)$ with $L^{l}$ in the bordism of $F_{\xi}$, then the new element is represented by

$$
M \times L \times I \stackrel{\pi}{\longrightarrow} M \times I \stackrel{H}{\longrightarrow} T(\xi)
$$

where we use a product triangulation on $M \times L$. Thus we may shift $H \circ \pi$ to $H^{1} \circ \pi$ which is s.f.h.t. in the product triangulation. The $G$-framed PD space in $M \times L \times\{1\}$ is $(Y \times L, t)$. This shows that the surgery problem is just crossed with $L$.

The product formulae in (iv) now follow from those in [15], [22], and [23] for ordinary surgery problems.

2.6. We have already observed that the open covers which we are considering are natural with respect to $T(f)$ where $f$ is a map of spherical fiber spaces which is simplicial on the base. From this 
it follows easily that the homomorphirms on $F_{\xi}$ are also natural with respect to such maps.

3. The obstructions to global transversality. Let $\xi^{q} \rightarrow \beta \xi^{q}$ be an oriented spherical fibration, with $\pi_{1}\left(B \xi^{q}\right)=0$, and let $F_{\xi q} \rightarrow W_{\xi q} \rightarrow$ $T \xi^{q}$ be the fibration considered in $\S 2$. (We have suppressed the cover $\{V \rho\}$ of $T \xi^{q}$ from the notation.) In $\S 2$ we defined homomorphisms (if $q \geqq 3$ )

$$
\begin{aligned}
& \mathscr{O}: \Omega_{q+i}^{P L}\left(F_{\xi q}\right) \longrightarrow P_{i} \\
& \mathcal{O}_{n}: \Omega_{q+i}^{P L}\left(F_{\xi q}, Z / n\right) \longrightarrow P_{i} \otimes Z / n \\
& \widetilde{O}^{:} \eta_{q+2}^{P L}\left(F_{\xi q}\right) \longrightarrow P_{i} \otimes Z / 2 .
\end{aligned}
$$

In this section we will work in the stable range; that is, we assume $i<q-2$. We will use the homomorphisms $\mathscr{O}, \mathscr{O}_{n}, \tilde{\mathcal{O}}$ to define homomorphisms on the bordism of $T \xi^{q}$, which measure exactly the obstruction to global transversality, up to cobordism, of a map

$$
M^{q+i} \stackrel{f}{\longrightarrow} T \xi^{q} .
$$

Thus $S\left(M^{q+i}, f\right)$ is 0 if and only if $(M, f)$ is cobordant to a globally f.h.t. map. (See Def. 1.1.)

Stably, the fibration $F_{\xi} \rightarrow W_{\xi} \rightarrow T \xi^{q}$ is also a cofibration. Thus, there is a natural isomorphism of bordism groups $\pi: \Omega_{q+j}^{P L}\left(W_{\xi}, F_{\xi}\right) \rightarrow$ $\widetilde{\Omega}_{q+j}^{P L}\left(T \xi^{q}\right), j<q-2$. The map $\pi$ is described geometrically as follows. A relative bordism element $(M, \partial M) \rightarrow\left(W_{\xi}, F_{\xi}\right)$ is defined by giving, first, a strongly fiber homotopy transversal map $f: M \rightarrow T \xi$ and, secondly, a homotopy $F: \partial M \times I \rightarrow T \xi$ of $f \mid \partial M$ to the trivial map. We obtain a bordism element $(D(M), g)$ of $T \xi$ by doubling $M, D(M)=$ $M \bigcup_{\partial M}(-M)$ and defining $g: D(M) \rightarrow T \xi$ by setting $g \mid M$ to be $f$ and setting $\left.g\right|_{-M}$ to be $F$ on a collar $\partial M \times I \subset(-M)$ and the trivial map outside this collar $\partial M \times I \subset(-M)$ and the trivial map outside this collar. Isomorphisms $\pi_{n}: \Omega_{q+j}^{P L}\left(W_{\xi}, F_{\xi}, Z / n\right) \cong \Omega_{q+j}^{P L}\left(T \xi^{q}, Z / n\right)$ and $\tilde{\pi} ; \eta_{q+j}^{P L}\left(W_{\xi}, F_{\xi}\right) \stackrel{\sim}{\rightarrow} \tilde{\eta}_{q+j}^{P L}\left(T \xi^{q}\right)$ are similarly defined if $j \leqq q-2$.

We now define homomorphisms

$$
\begin{aligned}
s: & \widetilde{\Omega}_{q+i+1}^{P L}\left(T \xi^{q}\right) \longrightarrow P_{i} \\
s_{n}: & \widetilde{\Omega}_{q+i+1}^{P L}\left(T \xi^{q}, Z / n\right) \longrightarrow P_{i} \otimes Z / n \\
\widetilde{s}: & \widetilde{\Omega}_{q+i+1}^{P L}\left(T \xi^{q}\right) \longrightarrow P_{i} \otimes Z / 2
\end{aligned}
$$

to be the compositions $(i<q-3) s=o \partial \pi^{-1}: \widetilde{\Omega}_{q+i+1}^{P L}\left(T \xi^{q}\right) \rightarrow \Omega_{q+i+1}^{P L}\left(W_{\xi}, F_{\xi}\right) \rightarrow$ $\Omega_{q+i}^{P L}\left(F_{\xi}\right) \rightarrow P_{i}$ and similarly, $s_{n}=o_{n} \partial \pi_{n}^{-1}$ and $\widetilde{s}=\widetilde{o} \partial \tilde{\pi}^{-1}$.

LEMMA 3.1. If $f: \xi^{q} \rightarrow \eta^{q}$ is a map of spherical fibrations, then 
the diagram

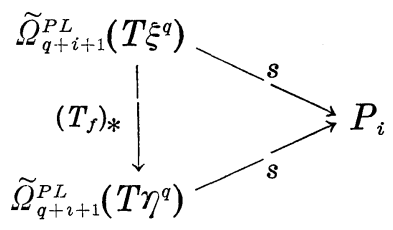

commutes, and similarly for $s_{n}$ and $\widetilde{s}$.

(ii) $s, s_{n}$, and $\widetilde{s}$ satisfy the same compatibity relations as $\mathcal{O}, \mathcal{O}_{n}$, and $\widetilde{O}$. (See Theorem 2.4(i), (ii).)

(iii) $s, s_{n}$ and $\widetilde{s}$ satisfy the same product formulae as $\mathcal{O}, \mathcal{O}_{n}$, and $\widetilde{O}$. (See Theorem 2.4(iv).)

(iv) $s, s_{n}$, and $\widetilde{s}$ are stable. That is, if $i<q-3$

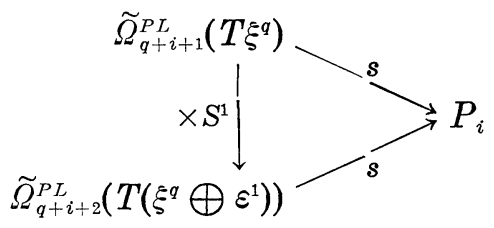

commutes, and similarly for $s_{n}$ and $\widetilde{s}$.

Proof. This is immediate from the definition of $s, s_{n}$, and $\widetilde{s}$ in terms of $\mathscr{O}, \mathscr{O}_{n}$, and $\tilde{\mathscr{O}}$.

Also, since the homomorphisms $o, o_{n}$ and $\widetilde{o}$ are defined as surgery obstructions, the homomorphisms $s, s_{n}$ and $\widetilde{s}$ are (indirectly) computed as surgery obstructions. For example, let $f:(M, \partial M) \rightarrow\left(W_{\xi}, F_{\xi}\right)$ represent an element of $\Omega_{q+i+1}^{P L}\left(W_{\xi}, F_{\xi}\right)$. This means we have a strongly transversal map $g: M \rightarrow T \xi$ and a homotopy to zero of $\left.g\right|_{\partial M}$. Let $(V, \partial V) \subset(M, \partial M)$ be the $(i+1)$-dimensional Poincaré pair $\left(g^{-1}\left(B \xi^{q}\right)\right.$, $g^{-1}\left(B \xi^{q}\right) \cap \partial M$ ), defined by the transversal map $g$. (We assume $B \xi^{q}$ is the mapping cylinder $M_{\hat{\xi}}$ of $\xi^{q}$.) Then

$$
s \pi([M, \partial M, f])=o\left(\left[\partial M,\left.f\right|_{\partial M}\right]\right) \in P_{i}
$$

is the surgery obstruction of degree one normal map $L^{\prime} \rightarrow L$, where $L$ is $\mathrm{PD}$ cobordant to $\partial V$ and $L^{\prime}$ is a $P L$ manifold which is a boundary. (See Note 2.5.) Thus both the domain and range of the surgery problem used to compute $s(x), x \in \widetilde{\Omega}_{q+i+1}^{P L}\left(T \xi^{q}\right)$, are PD boundaries. We conclude, for example,

3.2. $s: \widetilde{\Omega}_{q+4 i+1}^{P L}\left(T \xi^{q}\right) \rightarrow P_{4 i}=Z$ is identically zero. In this case, $s$ is computed as the difference of indices of two oriented boundaries.

Similarly, both the domain and range of the surgery problem used to compute $s_{n}(y), y \in \Omega_{p+i+1}^{P L}\left(T \xi^{q}, Z / n\right)$ or $\widetilde{s}(z), z \in \widetilde{\eta}_{q+i+1}^{P L}\left(T \xi^{q}\right)$ are $\mathrm{PD}$ boundaries; in the first case, boundaries as $Z / n$ PD spaces and 
in the second case, boundaries as unoriented PD spaces.

REMARK 3.3. 3.2 could also be established by appealing to the fact that $\widetilde{\Omega}_{q+42+1}^{P L}\left(M S G_{q}\right)$ is finite and using naturality of $s$ with respect to bundle maps.

THEOREM 3.4(i). Suppose $f: M \rightarrow T \xi^{q}$ represents an element of $\widetilde{\Omega}_{q+i+1}^{P L}\left(T \xi^{q}\right)$ [resp. $\left.\widetilde{n}_{q+i+1}^{P L}\left(T \xi^{q}\right)\right] . \quad$ If $s([M, f])=0$ [resp. $\left.\widetilde{s}([M, F])=0\right]$, then $f: M \rightarrow T \xi^{q}$ is cobordant to a globally transversal map.

(ii) If $f: M \rightarrow T \xi^{q}$ represents an element of $\widetilde{\Omega}_{q+i+1}^{P L}\left(T \xi^{q}, Z / n\right), i$ even, [resp. $i$ odd] and $s_{n}([M, f])=0$ [resp. $\left.s\left(\left[\delta M,\left.f\right|_{\delta M}\right]\right)=0\right]$, then $f: M \rightarrow T \xi^{q}$ is cobordant to a globally transversal map.

Proof. Choose a relative bordism element $g:(N, \partial N) \rightarrow\left(W_{\xi}, F_{\xi}\right)$ such that $\pi([N, \partial N, g])=[M, f]$ where $\pi$ is the doubling construction defined above. $g$ corresponds to a map $G: \partial N \times I \rightarrow T \xi^{q}$ with $\left.G\right|_{\partial_{N} \times 0}$ strongly transversal and $\left.G\right|_{\partial_{N \times 1}}$ the trivial map. By assumption $0=s([M, F])=\mathscr{O}(\partial N \times I, G)$. Thus by Theorem 2.4(iii) we may assume that $G: \partial N \times I \rightarrow T \xi^{q}$ is globally transversal. It is then obvious (see figure below) that the induced map $\pi([N, \partial N, g])=g \cup G$ : $N \cup(-N) \rightarrow T \xi^{q}$ is globally transversal. This proves (i).

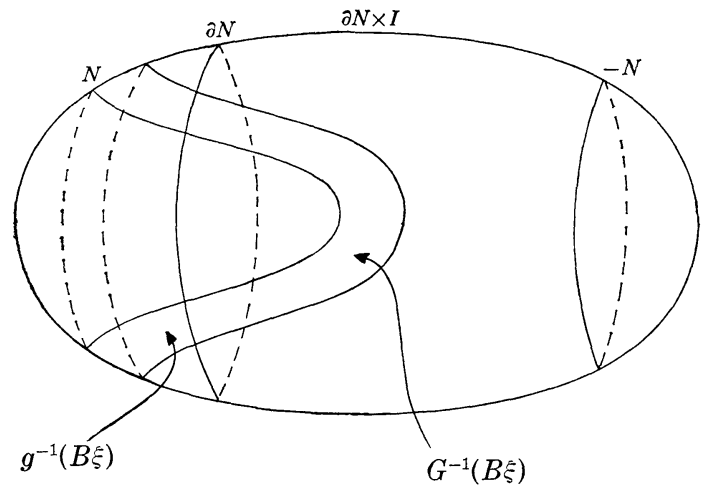

The proof of (ii) is essentially the same. There is the additional complexity that one must keep track of $\boldsymbol{Z} / n$ manifolds with boundary in $\Omega_{*}^{p L}\left(W_{\xi}, F_{\xi}, \boldsymbol{Z} / n\right)$, but we leave the details to the reader.

Our next goal will be to prove the converse of Theorem 3.4. In the process, we establish an alternate definition of the homomorphisms $s$ and $s_{n}$ on the bordism of $T \xi^{q}$, which is of independent interest.

There is a natural Pontrjagin-Thom isomorphism $p: \widetilde{\Omega}_{q+j}^{P L}\left(T \xi^{q}\right) \stackrel{\sim}{\rightarrow}$ $\pi_{3 q+j}\left(T \xi^{q} \Lambda M S P L_{2 q}\right), j<q-2$. Namely, given $f: M^{q+j} \rightarrow T \xi^{q}$, embed $M^{q+j} \subseteq S^{3 q+j}$ and cover $f$ by a map of bundles 


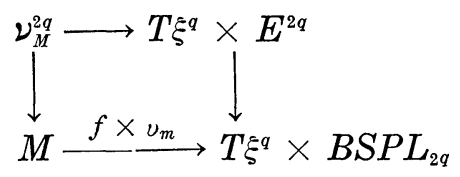

where $E^{2 q}$ is the universal $P L$ bundle. Now apply the Thom construction to obtain $S^{3 q+j} \rightarrow T \nu_{M}^{2 q} \rightarrow\left(T \xi^{q}\right)^{+} \Lambda M S P L_{2 q}$. Since $[M, f] \epsilon$ $\widetilde{\Omega}_{q+j}^{P L}\left(T \xi^{q}\right)=\operatorname{kernel}\left(\Omega_{q+j}^{P L}\left(T \xi^{q}\right) \rightarrow \Omega_{q+j}^{P L}(p t)\right)$, we obtain a well-defined element of $\pi_{3 q+j}\left(T \xi^{q} \Lambda M S P L_{2 q}\right)$.

Now, $T \xi^{q} A M S P L_{2 q}$ is itself a Thom space; namely, the Thom space of the Whitney sum $\xi^{q} \times E^{2 q} \rightarrow \xi^{q} \times B S P L_{2 q}$. From $\S 2$, there is a fibration $F_{\xi \times E} \rightarrow W_{\xi \times E} \rightarrow T \xi^{q} \Lambda M S P L_{2 q}$ and $\pi_{3 q+j}\left(F_{\xi \times E}\right)=P_{j}$. We thus define $s^{\prime}=\partial p: \widetilde{\Omega}_{q+i+1}^{P L}\left(T \xi^{q}\right) \rightarrow \pi_{3 q+i+1}\left(T \xi^{q} \Lambda M S P L_{2 q}\right) \rightarrow \pi_{3 q+i}\left(F_{\xi \times E}\right)=P_{i}$.

Similarly, there is an isomorphism

$$
p_{n}: \widetilde{\Omega}_{q+j}^{P L}\left(T \xi^{q}, Z / n\right) \stackrel{\sim}{\longrightarrow} \pi_{3 q+j}\left(T \xi^{q} \Lambda M S P L_{2 q}, Z / n\right)
$$

and we define $s_{n}^{\prime}=\partial p_{n}: \widetilde{\Omega}_{3 q+i+1}^{P L}\left(T \xi^{q}, Z / n\right) \rightarrow \pi_{3 q+i+1}\left(T \xi^{q} \Lambda M S P L_{2 q}, Z / n\right) \rightarrow$ $\pi_{3 q+i}\left(F_{\xi \times E}, Z / n\right)$. It is easy to see that if $n$ is odd $\pi_{3 q+i}\left(F_{\xi \times E}, Z / n\right)=$ $\boldsymbol{Z} / n, 0,0,0$ as $i \equiv 0,1,2,3(\bmod 4)$, respectively, and, if $n$ is even, $\pi_{3 q+i}\left(F_{\xi \times E}, \boldsymbol{Z} / n\right)=\boldsymbol{Z} / n, 0, \boldsymbol{Z} / 2, \boldsymbol{Z} / 2$ as $i \equiv 0,1,2,3(\bmod 4)$, respectively. Moreover

$$
\begin{aligned}
s^{\prime} & =0: \widetilde{\Omega}_{q+4 i+1}^{P L}\left(T \xi^{q}\right) \longrightarrow Z \\
s^{\prime} & =s_{2}^{\prime} \rho_{2}: \widetilde{\Omega}_{q+4 i-1}\left(T \xi^{q}\right) \longrightarrow \widetilde{\Omega}_{q+4 i-1}\left(T \xi^{q}, Z / 2\right) \longrightarrow Z / 2 \\
s_{n}^{\prime} & =s^{\prime} \delta: \Omega_{q+4 i-1}^{P L}\left(T \xi^{q}, Z / n\right) \longrightarrow \Omega_{q+4 i-1}^{P L}\left(T \xi^{q}\right) \longrightarrow Z / 2 .
\end{aligned}
$$

THEOREM 3.5 (i). $s=s^{\prime}: \widetilde{\Omega}_{q+j+1}^{P L}\left(T \xi^{q}\right) \longrightarrow P_{j}$.

(ii) $s_{n}=s_{n}^{\prime}: \widetilde{\Omega}_{q+j+1}^{P L}\left(T \xi^{q}, Z / n\right) \longrightarrow P_{j} \otimes Z / n, j$ even.

Proof. Statement (i) is trivial if $j \equiv 0(\bmod 4)$ since $s=s^{\prime}=0$, and if $j \equiv 2(\bmod 4)$, statement (i) follows from (ii).

To prove statement (ii) if $j \equiv 0(\bmod 4)$, we will show that if $f: M \rightarrow T \xi^{q}$ represents an element of $\widetilde{\Omega}_{q+4 i+1}^{P L}\left(T \xi^{q}\right)$ and $\left.f\right|_{\delta M}: \delta M \rightarrow T \xi^{q}$ is globally transversal, with $\left(\left.f\right|_{\delta M}\right)^{-1}\left(B \xi^{q}\right)=L^{4 i} \subset(\delta M)^{q+4 i}$ the associated $\mathrm{PD}$ space of dimension $4 i$, then $s_{n}([M, f])$ and $s_{n}^{\prime}([M, f]) \in \boldsymbol{Z} / n$ are determined by the index of $L^{4 i}$ modulo 8. (By 3.2 and 3.4(i), the hypothesis that $\left.f\right|_{\delta M}$ is globally transversal puts no restriction on $[M, f] \in \widetilde{\Omega}_{q+4 i+j}^{P L}\left(T \xi^{q}\right)$.) First, we show that index $\left(L^{4 i}\right)(\bmod 8)$ depends only on the bordism class of $\left[\delta M,\left.f\right|_{\delta M}\right]$.

From the definition of $p$ above, it is clear that $\alpha=p\left(\left[\delta M,\left.f\right|_{\delta M}\right]\right)$ : $S^{3 q+4 i} \rightarrow T \xi^{q} \Lambda M S P L_{2 q}$ is global transversal: in fact

$$
X^{4 i}=\alpha^{-1}\left(B \xi^{q} \times E^{2 q}\right) \subset S^{3 q+4 i} \quad \text { and } \quad L^{4 i}=\left(\left.f\right|_{\delta M}\right)^{-1}\left(B \xi^{q}\right) \subset(\delta M)^{q+4 i}
$$

are equivalent as PD spaces. By 1.3(3) and a slight generalization 
of 1.4, we can homotope $\alpha: S^{3 q+4 i} \rightarrow T \xi^{q} \Lambda M S P L_{2 q}$ to a strongly transversal map $\beta$ (see Def. 1.2) such that $Y^{4 i}=\beta^{-1}\left(B \xi^{q} \times E^{2 q}\right) \subset X^{4 i}$ and $Y^{4 i} \rightarrow X^{4 i}$ is a degree one normal map. In particular, index $\left(L^{4 i}\right)=$ index $\left(X^{4 i}\right) \equiv$ index $\left(Y^{4 i}\right)(\bmod 8)$. Moreover, it is not difficult to show using the obstruction theory of $\S 1$ that index $\left(Y^{4 i}\right)(\bmod 8)$ depends only on the homotopy class of the strongly transversal map $\beta: S^{3 q+4 i} \rightarrow$ $T \xi^{q} \Lambda M S P L_{2 q}$. (In fact, $Y^{4 i}$ is well-defined modulo cobordism of PD spaces and connected sum with the $G$-framed Milnor manifold $M^{4 n}$ of index 8.) We conclude from this paragraph the following.

LEMMA 3.6. If $g: N^{q+4 i} \rightarrow T \xi^{q}$ is globally transversal with $g^{-1}\left(B \xi^{q}\right)=K^{4 i} \subset N^{q+4 i}$ and if $[N, g]=0 \in \widetilde{\Omega}_{q+4 i}\left(T \xi^{q}\right)$, then index $\left(K^{4 i}\right) \equiv$ $0(\bmod 8)$.

Now we return to the $\boldsymbol{Z} / n$ bordism element $f: M \rightarrow T \xi^{q}$, with $\left(\left.f\right|_{\delta H}\right)^{-1}\left(B \xi^{q}\right)=L^{4 i}$. By definition, $s_{n}([M, f]) \in \boldsymbol{Z} / n$ is computed as the surgery obstruction of a normal map of $4 i$ dimensional $Z / n$ PD spaces, $W^{\prime} \rightarrow W$, (in fact, $W^{\prime}$ is a $P L$ manifold) where both $W^{\prime}$ and $W$ bound as $\boldsymbol{Z} / n$ objects. This means that $\delta W^{\prime}=\partial V^{\prime}, \delta W=\partial V$ and $W^{\prime} U_{\partial}\left(-n V^{\prime}\right)=$ $\partial Q^{\prime}, W \mathrm{U}_{\partial}(-n V)=\partial Q$, where $V^{\prime}, Q^{\prime}$ are $P L$ manifolds with boundary, $V, Q$ are PD spaces with boundary, $\operatorname{dim}\left(V^{\prime}\right)=\operatorname{dim}(V)=4 i$, and $\operatorname{dim}\left(Q^{\prime}\right)=$ $\operatorname{dim}(Q)=4 i+1$. Since the dimension is odd, we may assume that $\delta W^{\prime}=\partial V^{\prime} \rightarrow \partial V=\delta W$ is a homotopy equivalence. (There are no low dimensional problems since we are allowed cobordisms of both the domain $W^{\prime}$ and the range $W$ of the surgery problem $W^{\prime} \rightarrow W$.) Moreover, the closed $\mathrm{PD}$ space $K^{4 \imath}=V \bigcup_{\partial}\left(-V^{\prime}\right)$ can be identified with $\left(\left.g\right|_{\delta_{N}}\right)^{-1}\left(B \xi^{q}\right)$, where $g: N \rightarrow T \xi^{q}$ is a $Z / n$ bordism element cobordant to $f: M \rightarrow T \xi^{q}$. (Specifically, $[N, g]$ is constructed in terms of the map $\pi_{n}: \Omega_{q+4 i+1}\left(W_{\xi}, F_{\xi}, Z / n\right) \rightarrow \widetilde{\Omega}_{q+4 i+1}\left(T \xi^{q}, Z / n\right)$.) By Lemma 3.6, index $\left(L^{4 i}\right) \equiv$ index $\left(K^{4 i}\right)(\bmod 8)$. On the other hand, by the Novikov additivity property of the index, index $\left(K^{4 i}\right)=\operatorname{index}(V)-\operatorname{index}\left(V^{\prime}\right)$, and also $0=\operatorname{index}\left(\partial Q^{\prime}\right)=\operatorname{index}\left(W^{\prime}\right)-n$ index $\left(V^{\prime}\right)$ and $0=\operatorname{index}(\partial Q)=$ index $(W)-n$ index $(V)$. Thus we have the following equation

$$
\begin{aligned}
\operatorname{index}\left(W^{\prime}\right)-\operatorname{index}(W) & =n \operatorname{index}(V)-n \operatorname{index}\left(V^{\prime}\right) \\
& =n \operatorname{index}\left(K^{4 i}\right) .
\end{aligned}
$$

Since $s_{n}([M, f]) \in \boldsymbol{Z} / n$ is defined as the $\bmod n$ reduction of the integer $(1 / 8)$ (index $\left(W^{\prime}\right)$ - index $(W)$ ), we have proved the following.

LEMMA 3.7. If $f: M^{q+4 i+1} \rightarrow T \xi^{q}$ is a $Z / n$ bordism element and $\left.f\right|_{\text {ом }}$ is globally transveral with $L^{4 i}=\left(\left.f\right|_{{ }_{\delta M}}\right)^{-1}\left(B \xi^{q}\right)$, let $I\left(L^{4 i}\right)=$ index $\left(L^{4 i}\right)(\bmod 8) \in \boldsymbol{Z} / 8$. Then

(i ) if $n=2^{r}, s_{n}([M, f])=2^{r-3} I\left(L^{4 i}\right) \in Z / 2^{r}$, 
(ii) if $n$ is odd, $s_{n}([M, f])=0$.

(In 3.7(i), if $r<3, I\left(L^{4 i}\right) \in 2^{3-r} Z / 8 Z \subset Z / 8 Z$. Thus, $2^{r-3} I\left(L^{4 i}\right) \in Z / 2^{r}$ makes sense.)

Finally, $s_{n}^{\prime}([M, f])$ is also (indirectly) defined as the surgery obstruction of a normal map of $\boldsymbol{Z} / n$ P.D. spaces, via the identification $\pi_{3 q+4 i}\left(F_{\xi \times E}, \boldsymbol{Z} / n\right)=P_{4 i} \otimes \boldsymbol{Z} / n=\boldsymbol{Z} / n$. One can apply the same argument used in the proof of Lemma 3.7 to deduce that $s_{n}^{\prime}([M, f]) \in \boldsymbol{Z} / n$ is determined by $I\left(X^{4 i}\right) \in Z / 8$, where

$$
X^{4 i}=\alpha^{-1}\left(B \xi^{q} \times E^{q}\right), \alpha=p\left(\left[\delta M,\left.f\right|_{\delta M}\right]\right): S^{3 q+4 \imath} \longrightarrow T \xi^{q} \Lambda M S P L_{2 q} .
$$

But we already know from the discussion preceding Lemma 3.6 that index $\left(X^{4 i}\right)=\operatorname{index}\left(L^{4 i}\right)$. Thus, $s_{n}^{\prime}([M, f])=s_{n}([M, f]) \in Z / n$. This proves Theorem $3.5($ ii) if $j \equiv 0(\bmod 4)$.

If $f: M^{q+4 i-1} \rightarrow T \xi^{q}$ is a $Z / 2^{r}$ bordism element, $s_{2} r([M, f])$ and $s_{2}^{\prime} r([M, f]) \in \boldsymbol{Z} / 2$ depend only on the $Z / 2$ reduction of $[M, f]$. That is, $s_{2} r([M, f])=s_{2} \rho_{2}([M, f])$ and $s_{2}^{\prime} r([M, f])=s_{2}^{\prime} \rho_{2}([M, f]) \in Z / 2$. Thus we assume $M$ is a $Z / 2$ manifold. Let $N^{6}$ be a $Z / 26$-manifold with $d\left(\delta N^{6}\right)=1 \in Z / 2$. By Lemma 3.1(iii) and Theorem 2.4(iv), $S_{2}([M, f][N])=$ $s_{2}([M, f]) \cdot d\left(\delta N^{6}\right)=s_{2}([M, f]) \in Z / 2$. Also from Theorem 2.4(iv) and the definition of $s_{2}^{\prime}, s_{2}^{\prime}\left([M, f][N]=s_{2}^{\prime}([M, f]) \cdot d\left(\delta N^{6}\right)=s_{2}^{\prime}([M, f]) \in Z / 2\right.$. Thus the case $j \equiv 2(\bmod 4)$ of Theorem 3.5(ii) follows from the case $j \equiv 0(\bmod 4)$ considered above. This completes the proof of Theorem 3.5 .

We have now developed sufficient machinery to prove the converse of Theorem 3.4, in most cases.

THEOREM 3.8. (i) If $[M, f] \in \widetilde{\Omega}_{q+j+1}^{P L}\left(T \xi^{q}\right)\left[\operatorname{resp} . n_{q+j+1}^{P L}\left(T \xi^{q}\right)\right]$ and $f: M \rightarrow$ $T \xi^{q}$ is globally transversal, then $s([M, f])=0[$ resp. $\widetilde{s}([M, f])=0)$. (ii) If $[M, f] \in \widetilde{\Omega}_{q+j+1}^{P L}\left(T \xi^{q}, Z / n\right), j$ even, [resp. $j$ odd] and $f: M \rightarrow T \xi^{q}$ is globally tranversal, then $s_{n}([M, F])=0$ [resp. $\left.s\left(\left[\delta M,\left.f\right|_{\delta M}\right]\right)=0\right]$.

Proof. We will postpone the unoriented case of 3.8(i) until Chapter IV. The oriented case of 3.8(i) has content only if $j \equiv$ $2(\bmod 4)$, and follows from $3.8($ ii) with $n=2, j \equiv 2(\bmod 4)$.

To prove 3.8(ii) with $j \equiv 0(\bmod 4)$, we observe that if $f: M^{q+4 i+1} \rightarrow$ $T \xi^{q}$ is globally transversal, with $f^{-1}\left(B \xi^{q}\right)=K^{4 i+1} \subset M^{q+4 i+1}$, a $\boldsymbol{Z} / n \mathrm{PD}$ space with $\delta K^{4 i+1}=L^{4 i} \subset \delta M$, then $0=\operatorname{index}\left(\delta K^{4 i+1}\right)=n$ index $\left(L^{4 i}\right) \in Z$, hence index $\left(L^{4 i}\right)=0$. Thus, by Lemma 3.7, $s_{n}([M, f])=0$. The case $j \equiv 2(\bmod 4)$ is deduced from the case $j \equiv 0(\bmod 4)$ by crossing with $N^{6}$ where $d\left(\delta N^{6}\right)=1 \in \boldsymbol{Z} / 2$, just as in the proof of Theorem 3.5. The case $j$ odd of 3.8(ii) follows from 3.8(i).

REMARK 3.9. Lemmas 3.6 and 3.7, along with the trick of crossing 
with $N^{6}, d\left(\delta N^{6}\right)=1 \in \boldsymbol{Z} / 2$, imply that the homomorphisms

$$
s_{n}: \widetilde{\Omega}_{q+i+1}^{P L}\left(T \xi^{q}, Z / n\right) \longrightarrow P_{i} \otimes Z / n
$$

(and hence also $s: \widetilde{\Omega}_{q+i+1}^{P L}\left(T \xi^{q}\right) \rightarrow P_{i}$ ) are independent of the cover $\left\{V_{\rho}\right\}$ of $T \xi^{q}$ which occurs implicity in the original definition of $s_{n}$. We will prove in Chapter IV that $\widetilde{s}: \tilde{\eta}_{q+i+1}\left(T \xi^{q}\right) \rightarrow P_{i} \otimes Z / 2$ is also independent of the cover $\left\{V_{\rho}\right\}$.

We have shown above that if $f: M^{q+4 n} \rightarrow T \xi^{q}$ is a map, $M$ a closed oriented manifold, then $f: M \rightarrow T \xi$ is cobordant to a globally transversal map $g: N \rightarrow T \xi\left(3.2\right.$ and Theorem 3.4) and, moreover, if $L^{4 n}=$ $g^{-1}\left(B \xi^{q}\right) \subset N^{q+4 n}$, index $\left(L^{4 n}\right) \in Z / 8$ is an invariant of $[M, f] \in \Omega_{q+4 n}^{P L}\left(T \xi^{q}\right)$ (Lemma 3.6). However, we have given no indication of how one might compute index $\left(L^{4 n}\right) \in Z / 8$. Similarly, we have defined

$$
s_{n}: \Omega_{q+i+1}^{P L}\left(T \xi^{q}, \boldsymbol{Z} / n\right) \longrightarrow P_{i} \otimes Z / n
$$

and proved that $s_{n}$ is exactly the obstruction to global transversality, up to cobordism (Theorem 3.8). But we have not discussed how $s_{n}\left(P^{q+i+1}, h\right) \in P_{i} \otimes Z / n$ might be computed if $h: P^{q+i+1} \rightarrow T \xi^{q}$ is a $Z / n$ manifold, although Lemma 3.7 reduces this question to the evaluation of an index. The following discussion remedies these defects to some extent, and will prove useful in $\S 9$.

Let $\pi: \xi^{q} \rightarrow B \xi^{q}$ be a spherical fibration. Let $M_{\xi} \subset T \xi^{q}$ be the mapping cylinder of $\pi$; that is, $M_{\xi}=\xi^{q} \times[0,1] \bigcup_{\pi \mid \xi \times 0} B \xi^{q}$. Let $M_{\xi}^{\prime} \subset M_{\xi}$ be a "smaller" mapping cylinder. Say $M_{\xi}^{\prime}=\xi^{q} \times[0,1 / 2] \bigcup_{\pi \mid \xi \times 0} B \xi^{q}$. It makes sense to say that a map is transversal with respect to either $M_{\xi}$ or $M_{\xi}^{\prime}$ (see Definition 1.1).

Definition 3.10. Let $f: M^{q+i} \rightarrow T \xi^{q}$ be a map, $M$ a closed manifold. A partition of $f: M \rightarrow T \xi$ consists of two codimension zero submanifolds $U_{1}$ and $U_{2}$ of $M$ such that $M=U_{1} \bigcup_{\partial} U_{2}, \partial U_{1}=U_{1} \cap U_{2}=$ $\partial U_{2}$, and such that $\left.f\right|_{U_{1}}: U_{1} \rightarrow T \xi$ is transversal with respect to $M_{\xi}$ and $\left.f\right|_{U_{2}}: U_{2} \rightarrow T \xi$ is transversal with respect to $M_{\xi}^{\prime}$.

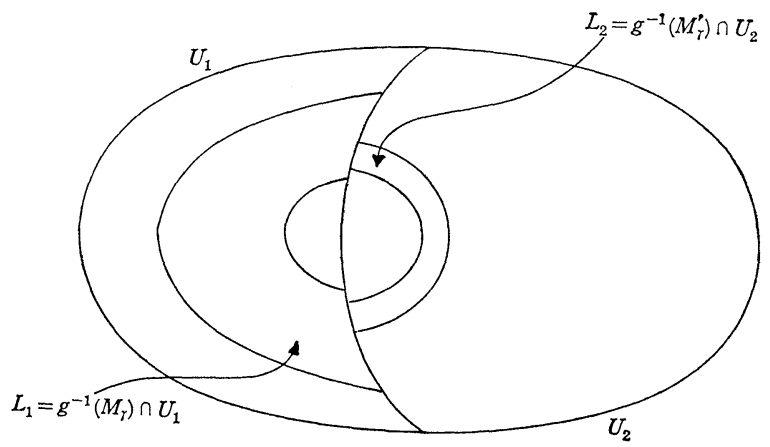


Let $L_{1}=f^{-1}\left(M_{\xi}\right) \cap U_{1}, L_{2}=f^{-1}\left(M_{\xi}^{\prime}\right) \cap U_{2}$, as in the figure. Thus $L_{j}$ is an $i$-dimensional PD space with boundary, $j=1,2$. By Lemma 1.4, the inclusion $\partial L_{2} \rightarrow \partial L_{1}$ is naturally a degree one normal map of $\mathrm{PD}$ spaces.

Let us assume $i=4 n, f: M^{q+4 n} \rightarrow T \xi^{q}$ partitioned as above. Then there is defined an invariant $\Theta\left(\partial L_{2} \rightarrow \partial L_{1}\right) \in \boldsymbol{Z} / 8$, [15], [4], [13]. Namely, let $K_{2 n-1}$ be the torsion subgroup of kernel $\left(H_{2 n-1}\left(\partial L_{2}, Z\right) \rightarrow\right.$ $\left.H_{2 n-1}\left(\partial L_{1}, Z\right)\right)$. The normal bundle map $\nu_{\partial L_{2}} \rightarrow \nu_{\partial L_{2}}$ covering $\partial L_{2} \rightarrow \partial L_{1}$ can be used to define a quadratic function $\psi: K_{2 n-1} \rightarrow Q / Z$, refining the natural linking pairing on $K_{2 n-1}$. Let $\Theta\left(\partial L_{2} \rightarrow \partial L_{1}\right)=\operatorname{Arf}(\psi) \in \boldsymbol{Z} / 8$.

If $W^{4 n} \rightarrow \partial L_{1}$ is any normal cobordism between $\partial L_{2} \rightarrow \partial L_{1}$ and a homotopy equivalence, then $\Theta\left(\partial L_{2} \rightarrow \partial L_{1}\right)=\operatorname{index}\left(W^{4 n}\right) \in \boldsymbol{Z} / 8$, Thus if we could do "Poincaré surgery" on $\partial L_{2} \rightarrow \partial L_{1}$ to a homotopy equivalence, we could prove that $f=f_{0}: M^{q+4 n} \rightarrow T \xi^{q}$ is homotopic to a transversal map $f_{1}: M^{q+4 n} \rightarrow T \xi^{q}$, and, if $L^{4 n}=f_{1}^{-1}\left(B \xi^{q}\right)$, then index $\left(L^{4 n}\right)=$ index $\left(L_{1}\right)+$ index $\left(L_{2}\right)-\Theta\left(\partial L_{2} \rightarrow \partial L_{1}\right) \in \boldsymbol{Z} / 8$. We assert that even without Poincaré surgery we can establish this last formula. That is,

LEMMA 3.11. Let $M^{q+4 n}=U_{1} \bigcup_{\partial} U_{2}$ be a partition as above, $L_{i}^{4 n} \subset U_{i}$. If $f: M^{q+4 n} \rightarrow T \xi^{q}$ is bordant to a transversal map $g: N^{q+4 n} \rightarrow T \xi^{q}$, with $L^{4 n}=g^{-1}\left(B \xi^{q}\right) \subset N^{q+4 n}$, then index $\left(L^{4 n}\right)=i n d e x\left(L_{1}\right)+\operatorname{index}\left(L_{2}\right)-$ $\Theta\left(\partial L_{2} \rightarrow \partial L_{1}\right) \in \boldsymbol{Z} / 8$.

Proof. The idea is the same as that used in the proof of Lemma 3.6. Namely, by a Pontrjagin-Thom construction, we define $p=$ $p([M, f]): S^{3 q+4 n} \rightarrow T \xi^{q} \Lambda M S P L_{2 q}$. Moreover, we preserve the partition, that is, we embed $\left(M^{q+4 n} ; U_{1}, U_{2}\right)\left(S^{3 q+4 n} ; D_{+}, D_{-}\right)$, where $D_{ \pm}$are upper and lower hemispheres. It is easy to see that then $p: S^{3 q+4 n} \rightarrow$ $T \xi^{q} \Lambda M S P L_{2 q}$ is partitioned, $S^{3 q+4 n}=D_{+} \bigcup_{\partial} D_{-}$, with the same transversal inverse images $L_{1}^{4 n} \subset D_{+}^{3 q+4 n}, L_{2}^{4 n} \subset D_{-}^{3 q+4 n}, \partial L_{2} \subset \partial L_{1} \subset S^{3 q+4 n-1}=$ $D_{+} \cap D_{-}$. (Strictly speaking, $L_{1}^{4 n}$ and $L_{2}^{4 n}$ are replaced by their thickenings.)

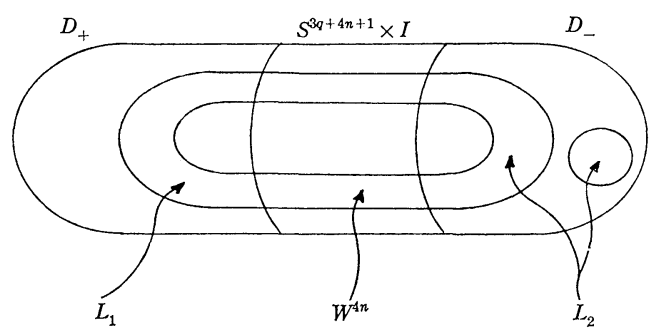

But we know that the two transversal maps $S^{3 q+4 n-1} \rightrightarrows T \xi^{q} \Lambda M S P L_{2 q}$ 
defined by $\partial L_{2} \subset S^{3 q+4 n-1}$ and $\partial L_{1} \subset S^{3 q+4 n-1}$ are homotopic. Moreover, there is no obstruction in this dimension to deforming a homotopy, rel the boundary, to a transversal homotopy, with transversal inverse image $W^{4 n} \subset S^{3 q+4 n-1} \times I$. (See figure above.) Then index $\left(W^{4 n}\right)=$ $\Theta\left(\partial L_{2} \rightarrow \partial L_{1}\right)$ and the lemma follows since index $\left(L^{4 n}\right)=\operatorname{index}\left(L_{1}\right)+$ index $\left(L_{2}\right)$ - index $(W)$.

Lemma 3.11 reduces certain index computations to computation of a $\Theta$-invariant. We state here some product formulae for evaluating $\Theta$ which will be used in $\S 9$. For proofs see [14], [5].

LEMma 3.12. (i) Let $f: M^{a} \rightarrow N^{a}$ be a degree one normal map of closed $\mathrm{PD}$ spaces, and let $L^{b}$ be a closed $\mathrm{PD}$ space, $a+b \equiv 3(\bmod 4)$. Then $\Theta\left(M^{a} \times L^{b} \rightarrow N^{a} \times L^{b}\right)=0$ if $a \equiv 0, b \equiv 3(\bmod 4) \Theta\left(M^{a} \times L^{b} \rightarrow N^{a} \times L^{b}\right)=$ $4 S_{k}\left(M^{a} \rightarrow N^{a}\right) \cdot d\left(L^{b}\right)$ if $a \equiv 2, b \equiv 1(\bmod 4)$. (Here $S_{k}\left(M^{a} \rightarrow N^{a}\right) \in Z / 2$ is the Kervaire obstruction, which is defined homotopy theoretically, $d\left(L^{b}\right) \in Z / 2$ is the de Rham invariant.)

(ii) Let $f: p^{a} \rightarrow Q^{a}$ be a normal map of $\boldsymbol{Z} / 2^{r}$ and $4: \boldsymbol{Z} / 2 \rightarrow \boldsymbol{Z} / 8$ $\mathrm{PD}$ spaces, and let $R^{b}$ be a $Z / 2^{r} \mathrm{PD}$ space, $a+b \equiv 0(\bmod 4)$. Form the normal map of closed $\mathrm{PD}$ spaces $\delta(P \otimes R)=P \times \delta R \bigcup_{(2 \hat{o} P \times \delta R)} \delta P \times R$

$$
\stackrel{(f \times 1) \cup(\delta f \times 1)}{\longrightarrow} \delta(Q \times R)=Q \times \delta R \underset{(2 \delta Q \times \dot{\delta} R)}{\bigcup} \delta Q \times R .
$$

Then

$$
\begin{gathered}
\Theta\left(\delta\left(P^{a} \otimes R^{b}\right) \longrightarrow \delta\left(Q^{a} \otimes R^{b}\right)\right)=0 \text { if } a \equiv 0, b \equiv 0(\bmod 4) \\
\Theta\left(\delta\left(P^{a} \otimes R^{b}\right) \longrightarrow \delta\left(Q^{a} \otimes R^{b}\right)\right)=0 \text { if } a \equiv 1, b \equiv 3(\bmod 4) \\
\Theta\left(\delta\left(P^{a} \otimes R^{b}\right) \longrightarrow \delta\left(Q^{a} \otimes R^{b}\right)\right)=4 d(\delta R) S_{k}\left(p^{a} \longrightarrow Q^{a}\right) \\
\text { if } a \equiv 2, b \equiv 2(\bmod 4) \\
\Theta\left(\delta\left(P^{a} \otimes R^{b}\right) \longrightarrow \delta\left(Q^{a} \otimes R^{b}\right)\right)=4 d\left(R^{b}\right) S_{k}(\delta P \rightarrow \delta Q) \\
\text { if } a \equiv 3, b \equiv 1(\bmod 4) .
\end{gathered}
$$

Now let $f: P^{q+\imath+1} \longrightarrow T \xi^{q}$ be a $Z / n$ manifold.

Definition 3.13. A $Z / n$ partition of $(P, f)$ consists of two codimension zero $Z / n$ submanifolds with boundary, $U_{1}$ and $U_{2} \subset P$, such that $P=U_{1} \bigcup_{\hat{\partial}} U_{2}, \delta P=\delta U_{1} \bigcup_{\hat{\partial} \hat{o}} \delta U_{2},\left.f\right|_{U_{1}}: U_{1} \rightarrow T \xi^{q}$ is transversal with respect to the mapping cylinder $M_{\xi} \subset T \xi$ and $\left.f\right|_{U_{2}}: U_{2} \rightarrow T \xi^{q}$ is transversal with respect to $M_{\xi}^{\prime} \subset M_{\xi}$.

The transversal inverse images $L_{1}^{i+1}=f^{-1}\left(M_{\xi}\right) \cap U_{1}$ and $L_{2}^{i+1}=$ $f^{-1}\left(M_{\xi}^{\prime}\right) \cap U_{2}$ are also $\boldsymbol{Z} / n$ manifolds with boundary. Moreover, $\partial L_{2}=f^{-1}\left(M_{\xi}^{\prime}\right) \cap U_{1} \cap U_{2} \subset f^{-1}\left(M_{\xi}\right) \cap U_{1} \cap U_{2} \neq \partial L_{1}$, and the inclusion $\partial L_{2} \rightarrow \partial L_{1}$ is a degree one normal map of $i$-dimensional, $\boldsymbol{Z} / n \mathrm{PD}$ spaces. 
Given a normal map $M^{i} \rightarrow N^{i}$ of $Z / n$ PD spaces, we can define a "surgery obstruction" $S_{n}\left(M^{i} \rightarrow N^{i}\right) \in P_{i} \otimes Z / n$ as follows:

$$
\begin{aligned}
& S_{n}\left(M^{4 h} \longrightarrow N^{4 h}\right)=\frac{1}{8}\left(\operatorname{index}\left(M^{4 h}\right)-\operatorname{index}\left(N^{4 h}\right)-n \Theta(\delta M \longrightarrow \delta N)\right) \in \boldsymbol{Z} / n \\
& S_{n}\left(M^{4 h-2} \longrightarrow N^{4 h-2}\right)=S_{k}(M \longrightarrow N) \in \boldsymbol{Z} / 2 \otimes \boldsymbol{Z} / n .
\end{aligned}
$$

Thus $S_{n}$ is defined homotopy theoretically for PD spaces, without any knowledge of PD surgery. $S_{n}$ is an invariant of the normal bordism class of $M \rightarrow N$, and clearly vanishes if $M \rightarrow N$ is a homotopy equivalence.

THEOREM 3.14. If $P=U_{1} \bigcup_{\partial} U_{2}$ is a $Z / n$ partition of $f: P^{q+\imath+1} \rightarrow T \xi^{q}$, with transversal inverse images $L_{j}^{2+1} \subset U_{j}^{q+2+1}, j=1,2$, as above, then

$$
S_{n}\left(\left[p^{q+i+1}, f\right]\right)=S_{n}\left(\partial L_{2} \longrightarrow \partial L_{1}\right) \in P_{i} \otimes Z / n .
$$

Proof. If $i \equiv 0(\bmod 4)$ this follows from Lemma 3.11 , the definition of $S_{n}$ in terms of the index and $\Theta$, and the proof of Lemma 3.7. We leave the details for the reader.

If $i \equiv 2(\bmod 4)$, cross with $\left[L^{6}\right]$, where $d\left(\delta L^{6}\right)=1 \in Z / 2$ and use Lemmas 3.1(iii) and 3.12(ii).

We conjecture that given any $f: M^{q+\imath+1} \rightarrow T \xi^{q}, M$ a closed or a $Z / n$ manifold, partitions always exist. If so, we could then use $\mathrm{PD}$ surgery to prove that $S([M, f]) \in P_{i}$ or $S_{n}([M, f]) \in P_{i} \otimes Z / n$ was exactly the obstruction to homotoping $f: M^{q+i+1} \rightarrow T \xi^{q}$ to a transversal map.

4. Geometric bundle structures and fiber homotopy transversality. Let $\xi$ be a spherical fiber space, $\widetilde{T}(\xi)$ the total singular complex of the Thom space, $\bar{W}_{\xi}$ the complex of f.h.t. maps $\Delta^{i} \rightarrow T(\xi)$. We have a natural inclusion $\bar{W}_{\xi} \subseteq \widetilde{T}(\xi)$. The geometric realization of $\widetilde{T}(\xi)$ is canonically homotopy equivalent to $T(\xi)$. If $X \stackrel{f}{\rightarrow} \widetilde{T}(\xi)$, then a lift of $f$ through $\bar{W}_{\xi}$ is the same thing as a deformation of $f$ into the subcomplex $\bar{W}_{\xi}$, i.e., $F: X \times I \rightarrow \widetilde{T}(\xi)$ with $F_{0}=f$ and $F_{1}(X) \subset \bar{W}_{\xi}$. Two such lifts (or deformations) are equivalent (concordant) if and only if there is a homotopy between them which is constant on $X \times\{0\}$ and which keeps $X \times\{1\}$ in $\bar{W}_{\xi}$. We say two $P L$ structures are equivalent if they are concordant.

In [11] it is shown that an equivalence class of $P L$ structures for $\xi$ gives a well defined equivalence class of deformation retracts of $\widetilde{T}(\xi)$ into $\bar{W}_{\xi}$ (i.e., liftings of $\widetilde{T}(\xi) \stackrel{\text { Id }}{\longrightarrow} \widetilde{T}(\xi)$ ). These deformations are given by homotopies of $\Delta^{i} \rightarrow T(\xi)$ to a $P L$ transverse map. If we do this construction universally we have 


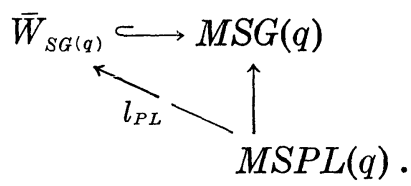

This same construction works relative to an open cover, and may be done so that all 2-dimensional preimages are homotopy equivalent to 2-manifolds. Thus we have

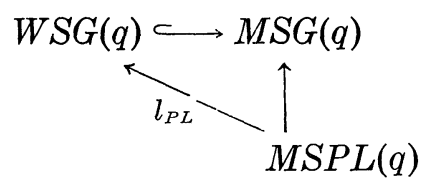

where $W S G(q)$ is the space of strongly f.h.t. maps small with respect to the cover of $B S G(q) \cdot l_{P L}$ is again define by $P L$ transversality and is well defined up to equivalence as a lift. In this section we shall construct a lifting of

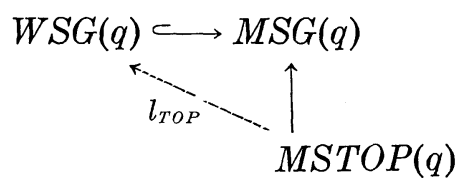

which will commute with $l_{P L}$ up to equivalence as a lift.

The lack of a topological transversality theorem in dimension 4 prevents us from defining this liftining directly. We use instead an enhanced version of the "crossing with $C P^{2}$ " trick of Sullivan, [23].

Let $M^{n}$ be a closed, oriented, connected, simly connected manifold. Define $\widetilde{T}(\xi)^{M^{n}}$ to be a semi-simplical complex whose $i$-simplices are maps $\Delta^{i} \times M^{n} \rightarrow T(\xi)$ which are contained in one of the sets in the open cover constructed in $\S 1$, i.e., all of $\Delta^{i} \times M^{n}$ is contained in one of the open sets. Let $W_{\xi}^{M^{n}}$ be all the above maps which are globally fiber homotopy transverse on $\Delta^{i} \times M^{n}$ and on all faces $\Delta^{i} \times M^{n}$. In addition we require that whenever the primage has dimension 2 , that the $\mathrm{PD}$ space with boundary be the homotopy type of a 2-manifold with boundary. Let $F_{\xi}(M)$ be the homotopy theoretic fiber of

$$
W_{\tilde{\xi}}^{M} \longrightarrow \widetilde{T}(\xi)^{M}
$$

THEOREM 4.1. (a) $\pi_{i}\left(F_{\xi} q\left(M^{n}\right)\right) \cong P_{i+n-q-1}$ if $q \geqq 3, q \geqq n-1$, and $M^{n}$ is closed, oriented, connected and simply connected

(b) if in addition $\operatorname{dim} M \equiv 0(4)$, then

$$
\times M^{n}: F_{\xi} \longrightarrow F_{\xi}\left(M^{n}\right)
$$

induces multiplication by $I\left(M^{n}\right), \pi_{*}\left(F_{\xi}\right) \rightarrow \pi_{*}\left(F_{\xi}\left(M^{n}\right)\right)$. 
Note. Theorem 2.4 is the special case of 4.1 when $M^{n}$ is a point.

Proof. (a) Given a map $\Delta^{i} \times M^{n} \rightarrow T\left(\xi^{q}\right)$ which is f.h.t. on $\partial \Delta^{i} \times M^{n}$; with $\xi^{q}$ admitting a $P L$ structure, use $P L$ transversality to produce a surgery problem of dimension $i+n-1-q$. Taking its obstruction gives a map $\pi_{2}\left(F_{\xi^{q}}\left(M^{n}\right)\right) \rightarrow P_{i+n-q-1}$. The same arguments as in 2.1 show that this map is an isomorphism for $i \neq 2$ and $i+n \neq 3+n$. When $i+n=3+n$ we use the fact that the two dimensional $\mathrm{PD}$ space in $\partial \Delta^{i} \times M^{n}$ is homotopy equivalent to a $P L$ manifold, to know that if the obstruction in $P_{*}$ vanishes then surgery to a homotopy equivalence is possible. When $i=2$, we have the extra difficulty that $\partial \Delta^{2} \times M^{n}$ is not simply connected. Thus our surgery problem is not necessarily one with trivial fundamental group but rather one with fundmental group $Z$. If $q \geqq n-1$, then the dimension of the surgery problem in $\partial \Delta^{2} \times M^{n}$ is $n+1-q \leqq 2$. Thus having fundamental group $Z$ causes no trouble.

(b) Obviously $\left(\times M^{n}\right)$ induces maps

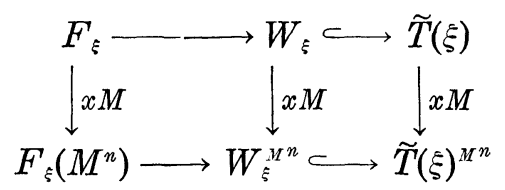

We see easily that the induced map on the fibers crosses the surgery problems involved with $M^{n}$. Thus it multiplies the surgery obstruction by the signature of $M^{n}$. Since $\pi_{*}\left(F_{\xi}\right)$ and $\pi_{*}\left(F_{\xi}(M)\right)$ are identified by surgery obstructions to $P_{*}$, this proves part (b).

COROLlary 4.2. If $M^{n}$ is closed, oriented, connected, simply connected and of index 1 , then $\left(\times M^{n}\right)$ induces a homotopy equivalence

$$
F_{\xi} \stackrel{\times M^{n}}{\longrightarrow} F_{\xi}\left(M^{n}\right) \text {. }
$$

Corollary 4.3. Given $X \stackrel{j}{\rightarrow} M S G$ and $M^{n}$ as above, then lifts of $j$ to $W_{S G}$ are in natural 1-1 correspondence with liftings of $\times M^{n} \circ j$ to $W_{S G}^{M}$.

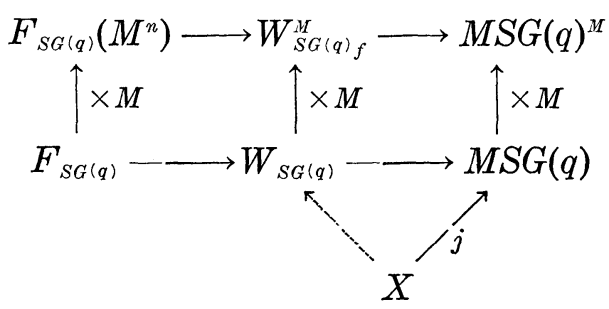


Now we use topological transversality to give a lifting of $M S T O P(q) \rightarrow M S G(q) \rightarrow M S G(q)^{C P^{2}}$. Use topological transversality inductively on simplies of dimension $\leqq q+3$ and then cross with $C P^{2}$. This gives a lifting on the $(q+3)$-skeleton of $M S T O P(q)$. On the $(q+4)$-skeleton we have

$$
\Delta^{q+4} \times C P^{2}, \stackrel{\eta}{\longrightarrow} \operatorname{MSG}(q)
$$

$\eta$ is topologically transverse on the boundary with preimage of dimension 7. Thus we can apply relative topological transversality to shift $\eta$ transverse. Continue in this manner up the skeleta of $\operatorname{MSTOP}(q)$, using only that $q+n+4>q+4$. This provides the lift, and a similar argument proves any two such lifts are equivalent. Thus by corollary 4.3 we have a well defined equivalence class of lifts

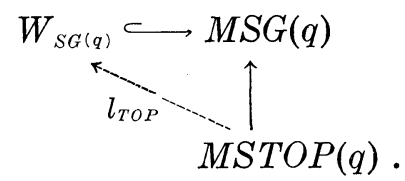

THEOREM 4.4. The lift induced on $\operatorname{MSPL}(q)$ by the above lift on $\operatorname{MSTOP}(q)$ agrees with any lift produced by $P L$ transversality.

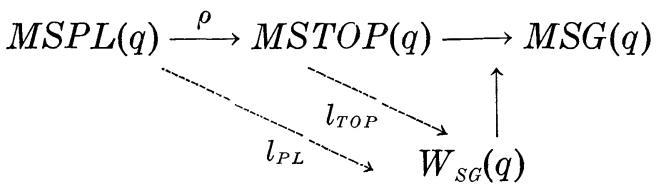

i.e., $l_{T O P} \circ \rho$ is equivalent as a lift to $l_{P L}$.

Proof. To show this we need only show that it is true after crossing with $C P^{2}$. After crossing with $C P^{2}$ compatibility of the lifts comes from the fact that $P L$ transversality and topological transversality are compatible.

In [11] it is proved that for a spherical fiber space $\xi^{q}$ over a four-connected space $X$, there is a 1-1 correspondence between equivalence classes of $P L$ structures on $\xi^{q}$ and liftings.

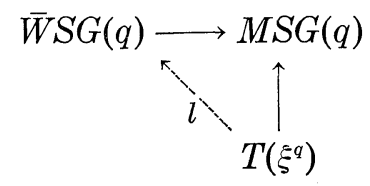

The correspondence is as follows. If $\widetilde{f}: X \rightarrow B S P L(q)$ is a lifting of $f: X \rightarrow B S G(q)$, the classifying map for $\xi^{q}$, then we form

$$
T\left(\xi^{q}\right) \stackrel{T(\tilde{f})}{\longrightarrow} M S P L(q) \stackrel{l_{P L}}{\longrightarrow} \bar{W}_{S G(q)}
$$


which is the lifting of $T(f): T(\xi) \rightarrow M S G(q)$. We now wish to strengthen this theorem by replacing $\bar{W}_{S G(q)}$ by $W_{S G}$.

Theorem 4.5. If $X$ is a connected, simply connected space, and $\xi^{q} \rightarrow X$ is a spherical fiber space which is classified by $f: X \rightarrow$ $B S G(q)$ then there is a natural one-to-one correspondence between topological structures on $\xi^{q}$ and liftings.

$$
l: T\left(\xi^{q}\right) \longrightarrow W S G \quad \text { of } \quad T(f): T(\xi) \longrightarrow M S G(q) .
$$

The correspondence is given by

$$
\begin{aligned}
\{\tilde{f}: X \longrightarrow \operatorname{BSTOP}(q) \text { lifting } f\} \\
\quad \longmapsto\left\{l_{T O P} \circ T(\widetilde{f}): T\left(\xi^{q}\right) \longrightarrow \operatorname{MSTOP}(q) \longrightarrow W S G(q)\right\}
\end{aligned}
$$

Proof. The argument proceeds exactly like the one in [11]. Namely we work one simplex at a time supposing we have a spherical fiber space $\xi^{q} \rightarrow D^{i+1}$, a topological structure $E \rightarrow \xi \mid \partial D^{i+1}$, and an extension of strong fiber homotopy transversality over all of $\xi^{q}$. Using this we show that the topological structure extends in a unique way so that topological transversality (after we cross with $C P^{2}$ ) agrees with the strong f.h.t. (after crossing with $C P^{2}$ ). Essentially we have shown that the obstruction to extending the f.h.t. over $\xi$ in all of $D^{i+1}$ is naturally identified with an element in $P_{\imath}$ and all such elements occur as obstructions. Of course $P_{i}$ is naturally identified with $\pi_{i}(G / T O P)$ and the topological structure on $\xi \mid \partial D$ gives an element in $\pi_{i}(G / T O P)$ which is the obstruction to extending the $P L$ structure over $\xi \rightarrow D^{i+1}$. What we show is that that these two obstructions are the same element just as in [11]. This argument works in all dimensions since $\pi_{i}\left(F_{\xi q}\right) \cong P_{i}-q-1$ in all dimensions; wheras the argument in [11] only works in dimensions $>4$ since the low homotopy groups of the fiber of $\bar{W}_{\xi q} \rightarrow T\left(\xi^{q}\right)$ are unknown.

\section{CHAPTER II}

5. $F_{\xi q}, G / T O P$, and localization at 2 . In this section we use the results of $\S 2$ and an argument similar to one in [11] to prove that $F_{\xi q}$ is naturally homotopy equivalent to copy of $G / T O P$ shifted $q$ dimensions. This result is a slight strengthening of the one in [11] in that the map constructed here is an isomorphism on all the homotopy groups where as the one in [11] is only an isomorphism on the high ( $\geqq 5$ ) dimensional groups. The argument is the same. The improvement in this version is that by strengthening the notion of fiber homotopy transversality in dimension 2 we get a hold on the low dimensional homotopy of $F_{\xi q}$. 
The results in [23] and [9] give a canonical equivalence $\Omega^{4}(G / T O P)$ with $G / T O P \times Z$. Let $G / \overparen{T O P}^{q}$ be the $q$-connected cover of $G / T O P$. Define $G / T O P\langle q\rangle$ to be $\Omega^{4 j-q}\left(\widetilde{G} / T O P^{4 j}\right)$ for any $j$ such that $4 j>q$. This is independent of $j$ (as long as $4 j>q$ ) by the fact that $\Omega^{4} G / T O P \cong$ $G / T O P \times Z . \quad \Omega G / T O P\langle q\rangle=G / T O P\langle q-1\rangle, G / T O P\langle 0\rangle=G / T O P$ and $G / T O P\langle q\rangle$ is $q$-connected.

THEOREM 5.1. (a) There exist canonical homomorphisms

(1) $\Omega_{*}\left(G / T O P\langle q\rangle \stackrel{\sigma}{\longrightarrow} P_{*-q}\right.$

(2) $\Omega_{*}(G / T O P\langle q\rangle ; Z / k) \stackrel{\sigma_{k}}{\longrightarrow} P_{*-q} \otimes Z / k$

satisfing (a) $\sigma$ and $\sigma_{k}$ are compatible with $Z \rightarrow Z / k$ and $\boldsymbol{Z} / k \rightarrow Z / k \cdot l$ if $*-q \equiv 0(\bmod 4)$.

(b) $\sigma$ is multiplicative with respect to the index

(c) $\sigma_{k}$ for $k$ odd is multiplicative respet to the index

(d) $\sigma_{2} k\left(\left(M^{m+q}, f\right) \otimes N^{n}\right)= \begin{cases}\sigma_{2} k(M, f) \cdot I(N) & n \equiv 0(4) \\ \sigma_{2} k(M, f) \cdot d(\delta N) & m \equiv n \equiv 2(4) \\ \sigma_{2}(f \mid \delta M) \cdot d(N) & m \equiv 3, n \equiv 1(4) \\ 0 & \text { otherwise }\end{cases}$

(b) $G / T O P\langle q\rangle$ is universal with respect to these homomorphisms in the category of $q$-connected spaces.

Note 1. When we say that $G / T O P\langle q\rangle$ is universal with respect to these homomorphisms in the category of $q$-connected spaces we mean that given $X q$-connected and homomorphisms

$$
\Omega_{*}(X ; \boldsymbol{Z}) \stackrel{\tau}{\longrightarrow} P_{*-q} \quad \text { and } \quad \Omega_{*}(X ; \boldsymbol{Z} / k) \stackrel{\tau}{\longrightarrow} P_{*-q} \otimes \boldsymbol{Z} / k
$$

satisfying (a) through (d) above, then there is a unique homotopy class of maps $X \stackrel{f}{\longrightarrow} G / T O P\langle q\rangle$ such that $f^{*} \sigma=\tau$ and $f^{*} \sigma_{k}=\tau_{k}$ for all $k$.

Note 2. It follows from the usual universality argument that the homomorphisms satisfying (a) through (d) above completely determine $G / T O P\langle q\rangle$.

Sketch of proof of 5.1 Case I $q=0$. The surgery obstruction maps, $S$ and $S_{k}$ give the required homomorphisms on $\Omega_{*}(G / T O P)$ and $\Omega_{*}(G / T O P ; Z / k)$. In [23] and [15] it is proved that they satisfy (a) through (d) in the theorem and that $G / T O P$ is universal with respect to them.

Case II $q=4 l . \quad G / T O P\langle 4 l\rangle=G / \widetilde{T O P}^{4 l}$. Thus we have a map 
$G / T O P\langle 4 l\rangle \stackrel{\pi}{\longrightarrow} G / T O P$. Let $\sigma=\pi^{*} S$ and $\sigma_{k}=\pi^{*} S_{k}$. The range of $\sigma$ is $P_{*}=P_{*-4 l}$ if $*>4 l$ and the range of $\sigma_{k}$ is $P_{*} \otimes \boldsymbol{Z} / k=P_{*-4 l} \otimes Z / k$ if $*>4 l$. Thus in dimensions greater than $4 l, \sigma$ and $\sigma_{k}$ have the correct ranges. In dimension less than or equal to $4 l$ the bordism groups of $G / T O P\langle 4 l\rangle$ are 0 and thus the ranges of $\sigma$ and $\sigma_{k}$ are 0 as they are supposed to be. $\sigma$ and $\sigma_{k}$ clearly satisfy (a)-(d) since $S$ and $S_{k}$ do.

To show that $\left\{G / T O P\langle 4 l\rangle, \sigma\right.$, and $\left.\sigma_{k}\right\}$ are universal let $\tau$ and $\tau_{k}$ be given a bordism of $X$ satisfying (a) through (d). Again identifying $P_{*-4 k}$ with $P_{*}$ for $* \geqq 4 l$ we use $\tau$ and $\tau_{k}$ to define a unique map, $f$

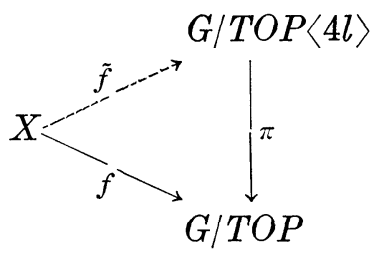

with $f^{*} S=\tau$ and $f^{*} S_{k}=\tau_{\kappa}$. This map factors uniquely through $G / T O P\langle 4 l\rangle$ since $X$ is $4 l$-connected.

Case III $q$ arbitrary. In this case we pick $4 l>q$ and use the fact that $\Omega^{4 l-q} G / T O P\langle 4 l\rangle=G / T O P\langle q\rangle$. We define $\sigma$ and $\sigma_{k}$ on $G / T O P\langle q\rangle$ to be

$$
\Omega_{*}\left(\Omega^{4 l-q} G / T O P\langle 4 l\rangle\right) \longrightarrow \Omega_{*-q+4 l}(G / T O P\langle 4 l\rangle) \stackrel{\sigma^{\prime}}{\longrightarrow} P_{*-q}
$$

and

$$
\begin{aligned}
& \Omega_{*}\left(\left(\Omega^{4 l-q} G / T O P\langle 4 l\rangle ; \boldsymbol{Z} / k\right) \longrightarrow \Omega_{*-q+4 l}(G / T O P\langle 4 l\rangle ; \boldsymbol{Z} / k)\right. \\
& \quad \stackrel{\sigma_{k}^{\prime}}{\longrightarrow} P_{*-q} \otimes \boldsymbol{Z} / k
\end{aligned}
$$

where $\sigma^{\prime}$ and $\sigma_{k}^{\prime}$ are the maps from case II on $G / T O P\langle 4 l\rangle$.

The homomorphisms are easily checked to satisfy (a)-(d) and to be be universal.

Apply 5.1 to $F_{\xi} q, q \geqq 3$, and the homomorphisms of Theorem 2.4 we have a canonical map

$$
F_{\xi q} \stackrel{\rho \xi}{\longrightarrow} G / T O P\langle q\rangle
$$

which is natural with respect to spherical fiber space maps which are simplicial on the base.

THEOREM 5.2. $\rho_{\xi}$ is a homotopy equivalence.

Proof. We have an isomorphism, $\mathscr{O} \circ h$ 


$$
\pi_{i}\left(F_{\xi^{q}}\right) \stackrel{h}{\longrightarrow} \Omega_{i}\left(F_{\xi^{q}}\right) \stackrel{\mathcal{O}}{\longrightarrow} P_{i-q} \cdot
$$

For the universal homomorphisms on $G / T O P\langle q\rangle$ the analogous statement is true. That is, the composition below is an isomorphism

$$
\pi_{i}\left(G / T O P\langle q\rangle \stackrel{h}{\longrightarrow} \Omega_{i}(G / T O P\langle q\rangle) \stackrel{\sigma}{\longrightarrow} P_{i-q},\right.
$$

Since $\left(\rho_{\xi}\right)^{*} \rho=\mathcal{O}$, we see that $\rho_{\xi}^{*}$ is an isomorphism on $\pi_{i}$ for all $i$.

Now we wish to specialize to the prime 2. According to [15] the homomorphisms gives cohomology classes in G/TOP, $\mathscr{L} \epsilon$ $H^{4^{*}}\left(G / T O P ; \boldsymbol{Z}_{(2)}\right)$ and $\mathscr{K} \in H^{4^{*+2}}(G / T O P ; \boldsymbol{Z} / 2)$. These classes are uniquely determined by cohomological formulae involving them which determines the homomorphism $S$ and $S_{2^{k}}$. The formulae are

(1) $\Omega_{4^{*}+2}(G /$ TOP $; \boldsymbol{Z} / 2) \rightarrow P_{4^{*}+2} \otimes \boldsymbol{Z} / 2=\boldsymbol{Z} / 2 \otimes \boldsymbol{Z} / 2=\boldsymbol{Z} / 2$ is given by

$$
s(M, f)=\left\langle f^{*} \mathscr{\&} \cdot V^{2}(M),[M]\right\rangle .
$$

(2) $\Omega_{4^{*}}(G / T O P) \stackrel{S}{\longrightarrow} P_{4^{*}}=\boldsymbol{Z}$ and $\Omega_{4^{*}}\left(G / T O P ; \boldsymbol{Z} / 2^{k}\right) \stackrel{S_{2^{k}}}{\longrightarrow} P_{4^{*}} \otimes Z / 2^{k}$

$=\boldsymbol{Z} / 2^{k}$ are given by $S(M, f)=\left\langle f^{*} \mathscr{L} \cdot \mathscr{L}_{M},[M]\right\rangle$

$+\left\langle\delta^{*}\left(f^{*} \mathscr{K} \cdot V S q^{1} V(M)\right),[M]\right\rangle$

Here $\mathscr{L}_{M}$ is a $\boldsymbol{Z}_{(2)}$ characteristic class of $M$ lifting the rational Hirzebruch $\mathscr{L}$-class, $V S q^{1} V(M)$ means the $\boldsymbol{Z} / 2$ characteristic class

$$
\left(\sum V_{i}(M)\right)\left(\sum S q^{1} V_{i}(M)\right)
$$

and $\delta^{*}$ is the $Z / 2$ integral Bockstein. See [23] and [15] for proofs.

There are analogous classes and formulae $G / T O P\langle q\rangle$. The classes in $G / T O P\langle 4 l\rangle$ are obtained by pulling back the ones in $G / T O P$ under the natural map $G / T O P\langle 4 l\rangle \stackrel{\pi}{\longrightarrow} G / T O P$. The classes and homomorphisms still satisfy the same formulae by naturality. The classes in $G / T O P\langle q\rangle$ are then obtained from those in $G / T O P\langle 4 j\rangle 4 j>q$, by looping. Once again since both the classes and homomorphisms are obtained in this way the formulae are still satisfied in $G / T O P\langle q\rangle$. Using $\rho_{\xi^{1}}: F_{\xi^{q}} \rightarrow G / T O P\langle q\rangle$ to pull back the classes to $F_{\xi^{q}}$, we see that we have classes $\mathscr{C} \in H^{4^{*}+2+q}\left(F_{\xi^{q}} ; \boldsymbol{Z} / 2\right)$ and $\mathscr{L} \in H^{4^{*+q}}\left(F_{\xi^{q}} ; \boldsymbol{Z}_{(2)}\right)$ satisfying:

THEOREM 5.3. (1) The obstruction homomorphism

$$
\odot: \Omega_{4^{*}+2+q}\left(F_{\xi q} ; \boldsymbol{Z} / 2\right)
$$

$\boldsymbol{Z} / 2$ is given by

$$
\mathscr{O}(M, f)=\left\langle f^{*} \mathscr{K} \cdot V^{2}(M),[M]\right\rangle .
$$

(2) The homomorphisms $\mathcal{O}: \Omega_{4^{*}+q}\left(F_{\xi q} ; \boldsymbol{Z}_{(2)}\right) \rightarrow \boldsymbol{Z}_{(2)}$ and 
$\left.\mathcal{O}: \Omega_{4^{*}+q}(F)_{\xi^{q}} ; \boldsymbol{Z} / 2^{k}\right) \rightarrow \boldsymbol{Z} / 2^{k}$ are given by

$$
\mathscr{O}(M, f)=\left\langle f^{*} \mathscr{L}, \mathscr{L}_{M},[M]\right\rangle+\left\langle\delta^{*}\left(f^{*} \mathscr{K} \cdot V S q^{1} V(M),[M]\right\rangle .\right.
$$

(3) $\mathscr{K}$ and $\mathscr{L} \in H^{*}\left(F_{\xi^{q}}\right)$ are uniquely determined by these formulae.

Now consider the fibration $F_{\xi^{q}} \rightarrow W_{\xi^{q}} \rightarrow T \xi^{q}$ of $\S 2$. In the stable range, $j<q-2$, we have a homomorphism $\tau: H^{q+j}\left(F_{\xi q}\right) \rightarrow H^{q+j+1}\left(T \xi^{q}\right)$, with any coefficients. Thus the cohomology classes $\mathscr{K} \in H^{4^{*-2+q}}\left(F_{\xi^{q}}, Z / 2\right)$ and $\mathscr{L} \in H^{4^{*}+q}\left(F_{\xi q}, \boldsymbol{Z}_{(2)}\right)$ determine classes $\tau(\mathscr{K}) \in H^{4^{*}-1+q}\left(T \xi^{q}, \boldsymbol{Z} / 2\right)$ and $\tau(\mathscr{L}) \in H^{4^{*+1+q}}\left(T \xi^{q}, \boldsymbol{Z}_{(2)}\right)$, if $4_{*}<q-3$. From the cohomology Thom isomorphism, we have $\tau(\mathscr{K})=\bar{\Phi}(\tilde{\mathscr{K}}), \tilde{\mathscr{K}} \in H^{4^{*-1}}\left(B \xi^{q}, \boldsymbol{Z} / 2\right)$ and $\pi(\mathscr{L})=$ $\bar{\Phi}(\tilde{\mathscr{L}}), \tilde{\mathscr{L}} \in H^{4_{*}+1}\left(B \xi^{q}, \boldsymbol{Z}_{(2)}\right)$. The classes $\tilde{\mathscr{L}}$ and $\tilde{\mathscr{L}}$ are natural with respect to bundle maps, since $\mathscr{K}$ and $\mathscr{L}$ have this property. Moreover, $\tilde{\mathscr{K}}$ and $\tilde{\mathscr{L}}$ are stable classes, since $\tau(\mathscr{K})$ and $\tau(\mathscr{L})$ commute with the suspension homomorphism $H^{j+q}\left(T \xi^{q}\right) \stackrel{\sim}{\longrightarrow} H^{j+q+1}\left(T\left(\xi^{q} \oplus \varepsilon^{1}\right)\right)$, $j<q-2$. Thus we have universal, stable characteristic classes $\tilde{\mathscr{K}} \in H^{4^{*-1}}(B S G, \boldsymbol{Z} / 2)$ and $\tilde{\mathscr{L}} \in H^{4^{*+1}}\left(B S G, \boldsymbol{Z}_{(2)}\right)$.

We can also define $\tilde{\mathscr{L}}$ and $\tilde{\mathscr{L}}$ in terms of the homomorphisms $s: \widetilde{\Omega}_{q+j+1}^{P L}\left(T \xi^{q}\right) \rightarrow P_{j}$ and $s_{2} r: \widetilde{\Omega}_{q+j+1}^{P L}\left(T \xi^{q}, Z / 2^{r}\right) \rightarrow P_{j} \otimes Z / 2^{r}$ of $\S 3$. Namely, $s$ and $s_{2} r$ are defined in terms of $\mathscr{O}$ and $\mathscr{O}_{2} r$ and $\mathscr{C} \in H^{q+4^{*-2}}\left(F_{\xi q}, Z / 2\right)$ and $\mathscr{L} \in H^{q+4^{*}}\left(F_{\xi^{q}} \boldsymbol{Z}_{(2)}\right)$ are defined by a cohomological formula for $\mathscr{O}$ and $\mathcal{O}_{2} r$ (see 5.3). Thus we have in the stable range

THEOREM 5.4. (1). The obstruction to global transversality

$$
s_{2}: \widetilde{\Omega}_{4^{*}-1+q}^{P L}\left(T \xi^{q}, Z / 2\right) \longrightarrow Z / 2
$$

is given by

$$
s_{2}([M, f])=\left\langle V^{2}(M) \cdot f^{*} \Phi(. \tilde{\check{\complement}}),[M]\right\rangle \in \boldsymbol{Z} / 2 .
$$

(2) The homomorphisms $s_{2} r: \widetilde{\Omega}_{4^{*}+1+q}^{P L}\left(T \xi^{q}, \boldsymbol{Z} / 2^{r}\right) \rightarrow \boldsymbol{Z} / 2^{r}$ are given by:

$$
\begin{aligned}
s_{2} r([M, f])= & \left\langle\mathscr{L}(M) \cdot f^{*} \Phi(\tilde{\mathscr{L}}),[M]\right\rangle \\
& +i\left\langle V S q^{1} V(\delta M) \cdot\left(\left.f\right|_{\delta M}\right)^{*} \Phi(\tilde{\mathscr{K}}),[\delta M]\right\rangle \in \boldsymbol{Z} / 2^{r},
\end{aligned}
$$

where

$$
i: \boldsymbol{Z} / 2 \longrightarrow \boldsymbol{Z} / 2^{r}
$$

is the inclusion.

(3) $\tilde{\mathscr{K}}$ and $\tilde{\mathscr{L}} \in H^{*}(B S G)$ are uniquely determined by these formulae.

6. Geometric bundle structures, localized at 2. In this section 
we continue to focus our attention on information at the prime 2 . We will show that if we use the classes $\tilde{\mathscr{K}}$ and $\tilde{\mathscr{L}}$ defined in $\S 5$ to form

$$
B S G_{(2)} \stackrel{\tilde{\mathscr{K}} \times \tilde{\mathscr{L}}}{\longrightarrow} \prod_{i \geq 1} K(Z / 2,4 i-1) \times K\left(Z_{(2)}, 4 i+1\right)
$$

then the homotopy theoretic fiber admits a natural equivalence with $B S T O P_{(2)}$ i.e.,

$$
\operatorname{BSTOP}_{(2)} \stackrel{\eta^{(2)}}{\longrightarrow} B S G_{(2)} \stackrel{\tilde{\mathscr{L}} \times \check{\mathscr{L}}}{\longrightarrow} \prod_{i \geqq 1} K(Z / 2,4 i-1) \times K\left(Z_{(2)}, 4 i+1\right)
$$

is a fibration where $\eta(2)$ is the localization at 2 of the natural map $\eta: B S T O P \rightarrow B S G$.

The map of $\operatorname{BSTOP}_{(2)}$ into the fiber of $(\tilde{\mathscr{K}} \times \tilde{\mathscr{L}})$ is obtained from the localization at 2 of the lifting

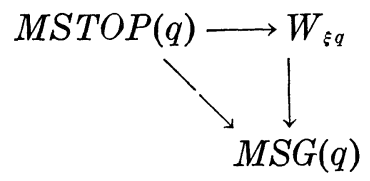

constructed in $\S 4$ by applying the Thom isomorphism. Thus

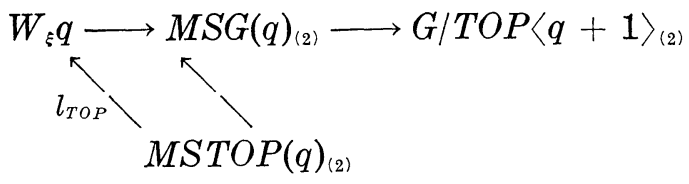

after applying the Thom isomorphism gives

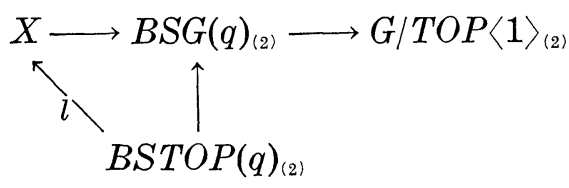

That $l$ is a homotopy equivalence is essentially a reformulation (by applying the Thom isomorphism) of the Theorem 4.5 that a topological structure for $\xi^{q}$ is equivalent to a section of

$$
W_{\xi q} \longrightarrow \widetilde{T}\left(\xi^{q}\right) \text {. }
$$

Let $X$ be the fiber of $(\tilde{\mathscr{L}} \times \tilde{\mathscr{L}})$

$$
X \longrightarrow B S G_{(2)} \stackrel{\tilde{\mathscr{K}} \times \tilde{\mathscr{E}}}{\longrightarrow} \prod_{i \geqq 1} K(Z / 2,4 i-1) \times K\left(\boldsymbol{Z}_{(2)}, 4 i+1\right) .
$$

Stabilizing the results of $\S 4$, we have a fibration of spectra

$$
F_{S G} \longrightarrow W_{S G} \longrightarrow M S G
$$


Since we are in a stable category, the fibration is also a cofibration and extends to the right as follows

$$
F_{S G} \longrightarrow W S G \longrightarrow M S G \longrightarrow \Sigma F_{S G} \cdot
$$

Localizing at 2 we have

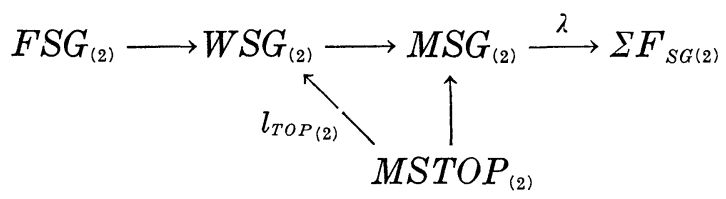

From this we shall produce a canonical lifting of $B S T O P_{(2)} \rightarrow B S G_{(2)}$ to $B S T O P_{(2)} \rightarrow X$, using a stronger version of the Thom isomophism.

Let $\Pi$ be a product of Eilenberg-MacLane spaces, and $\Pi\langle q\rangle$ another product which is $q$-connected and with $\Omega^{q} \Pi\langle q\rangle \stackrel{\sim}{\sim} \Pi$.

Then there is a map, well defined up to homotopy,

$$
K(Z, q) \times \Pi \stackrel{\rho}{\longrightarrow} \Pi\langle q\rangle
$$

given by cupping with $\iota_{q}$, the fundamental class of $K(Z, q)$. We may arange this map so that $\rho(* x \Pi)=*$.

Lemma 6.1. Let $\xi^{q} \rightarrow B$ be an oriented $q$-dimensional spherical fiber space. Then we have a natural homotopy equivalence

$$
\Pi^{B} \stackrel{\cup \text { Thom class }}{\longrightarrow}(\Pi\langle q\rangle, *)^{\left(T\left(\xi^{q}\right), *\right)} .
$$

NoTE. The statement on $\pi_{0}$ translates to $H^{*}(B) \stackrel{\text { UThom class }}{\longrightarrow}$ $H^{q+*}\left(T\left(\xi^{q}\right)\right)$ is an isomorphism.

Proof. Let $U \in H^{q}\left(T\left(\xi^{q}\right) ; Z\right)$ be the Thom class.

Let $\widetilde{U}:\left(T\left(\xi^{q}\right), *\right) \rightarrow(K(\boldsymbol{Z}, q), *)$ be a representative for $U$. Define $\Pi \stackrel{B}{\longrightarrow}(\Pi\langle q\rangle, *)^{\left(T\left(\xi^{q}\right), *\right)}$, by $(f: B \rightarrow \Pi) \mapsto\left[\left(D \xi^{q}\right), S\left(\xi^{q}\right)\right) \stackrel{\Delta}{\longrightarrow}\left(D\left(\xi^{q}\right), S\left(\xi^{q}\right)\right) \times$ $\left.D\left(\xi^{q}\right) \stackrel{1 \times \operatorname{proj}}{\longrightarrow}\left(D\left(\xi^{q}\right), S\left(\xi^{q}\right)\right) \times B \stackrel{\tilde{U} \times f}{\longrightarrow} K(Z, q) \times \Pi \stackrel{\rho}{\longrightarrow} \Pi\langle q\rangle\right] . \quad$ We identify $T\left(\xi^{q}\right)$ with $D\left(\xi^{q}\right) / S\left(\xi^{q}\right)$. Notice also that the base point of $T\left(\xi^{q}\right)$ goes to $\rho(*, \Pi)=*$. Thus we have a map $\Pi^{B} \stackrel{\mu}{\longrightarrow}(\Pi\langle q\rangle, *)^{\left.\left(T_{(\xi} q\right), *\right)}$.

On $\pi_{0}$ it is just the usual map $H^{*}(B) \stackrel{\cup \mathscr{\mathscr { C }}}{\longrightarrow} H^{*+q}\left(T\left(\xi^{q}\right)\right)$ and thus is an isomorphism. We now show that it is an isomorphism on $\pi_{2}$. Since $\Pi^{B}$ and $(\Pi\langle q\rangle, *)^{\left(T\left(\xi^{q}\right), *\right)}$ are $H$-spaces it suffices to show that $\mu_{*}:\left[S^{i}, \Pi^{B}\right] \rightarrow\left[S^{i},(\Pi\langle q\rangle, *)^{\left(T\left(\xi^{q}\right), *\right)}\right]$ is a bijection.

$$
\begin{aligned}
& {\left[S^{i}, \Pi^{B}\right]=\pi_{0}\left(\Pi^{B \times S^{i}}\right)=H^{*}\left(B \times S^{i}\right)} \\
& \left.\left[S^{i},(\Pi\langle q\rangle, *)^{\left(T\left(\xi^{\eta}\right), *\right)}\right]=\pi_{0}[\Pi\langle q\rangle, *)^{\left(T\left(\xi^{q}\right) \times S^{i}, * \times S^{i}\right)}\right]
\end{aligned}
$$


But

$$
\begin{aligned}
\left(T\left(\xi^{q}\right) \times S^{i}\right) /\left(* \times S^{i}\right) & =T\left(\xi^{q}\right) \wedge\left(S^{i+}\right)=T\left(\xi^{q}\right) \wedge\left(S^{i} \longrightarrow p t\right) \\
& =T\left(\xi^{q} \times 1_{S^{i}}\right),
\end{aligned}
$$

where $\xi^{q} \times 1_{S^{i}} \rightarrow B \times S^{i}$ is the product fiber space. Thus

$$
\left[S^{i},(\Pi\langle q\rangle, *)^{\left(T\left\langle\xi^{q}\right), *\right)}\right]=H^{*}\left(T\left(\xi^{q} \times 1_{S^{i}}\right)\right) .
$$

The map induced by $\mu^{*}$ from $H^{*}\left(B \times S^{i}\right)$ to $H^{*+q}\left(T\left(\xi^{q} \times 1_{S^{i}}\right)\right)$ is easily seen to be $U(U \otimes 1)$ which is the Thom class of this bundle. This proves the lemma.

We now apply this lemma to the fibration

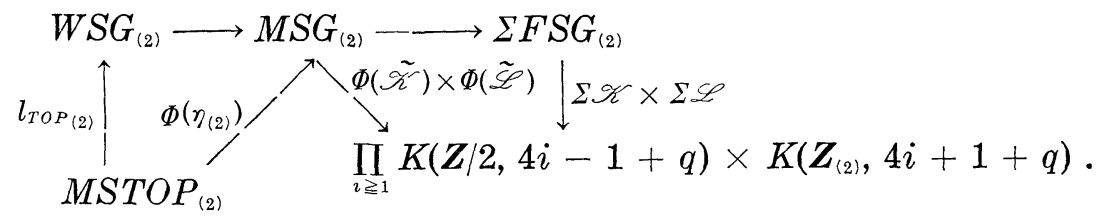

The lifting $l_{T O P(2)}$ is equivalent to a deformation of

$$
\Phi(\tilde{\mathscr{L}}) \times \Phi(\hat{\mathscr{L}}) \circ \Phi\left(\eta_{(2)}\right)=\Phi\left(\tilde{\mathscr{K}} \times \tilde{\mathscr{L}} \circ \eta_{(2)}\right) \text { to *. }
$$

The previous lemma applied to $\pi_{0}$ and $\pi_{1}$ of the mapping spaces tells us that $(\tilde{\mathscr{K}} \times \tilde{\mathscr{L}}) \circ \eta_{2}$

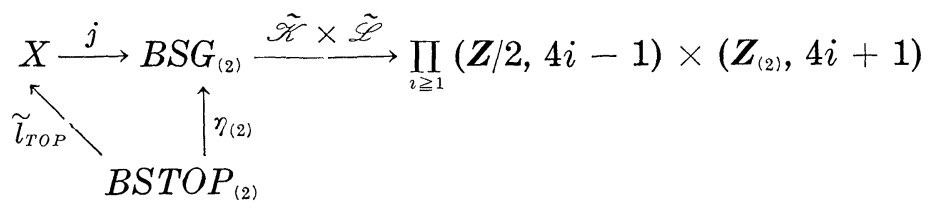

is homotopic to $*$, and, in fact picks out up to homotopy a homotopy $(\tilde{\mathscr{K}} \times \tilde{\mathscr{L}}) \circ \eta_{(2)}$ to $*$. This is equivalent to a lift of $\eta_{(2)}$ to the fiber $\boldsymbol{X}, \widetilde{l}_{\text {TOP }}: B S T O P_{(2)} \rightarrow \boldsymbol{X}$.

THEOREM 6.2. $\quad \tilde{l}_{\text {TOP }}$ is a homotopy equivalence.

Proof. Theorem 4.5 says that if $B$ is 1 -connected and $f: B \rightarrow$ $B S G$, then liftings of $f$ to $B S T O P$.

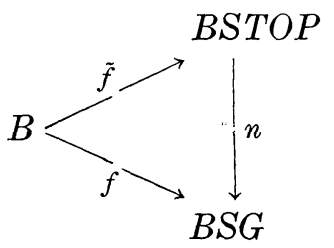

are in natural 1-1 correspondence with liftings $\mu$ of $T(f)$ 


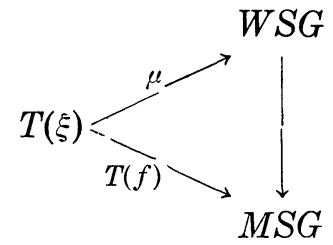

where $\xi$ is the spherical fiber space over $B$ induced by $f$. The correspondence is $\widetilde{f} \mapsto l_{T O P} \circ T(\widetilde{f})$.

Localizing at 2 , we see that liftings

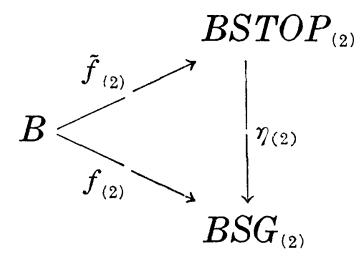

are in natural 1-1 correspondence with liftings

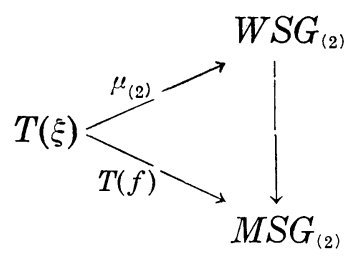

The correspondence is given by

$$
\tilde{f}_{(2)} \longrightarrow l_{T O P(2)} \circ T(\tilde{f})
$$

Let $S^{i} \stackrel{g}{\longrightarrow} X \stackrel{j}{\longrightarrow} B S G_{(2)} \stackrel{\tilde{\mathscr{K}} \times \tilde{\mathscr{L}}}{\longrightarrow} \Pi K(\boldsymbol{Z} / 2,4 i-1) \times K\left(\boldsymbol{Z}_{(2)}, 4 i+1\right)$. The previous lemma gives a lifting

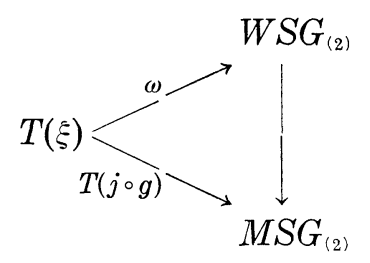

where $\rho$ is the bundle over $S^{2}$ induced by $j \circ g$. This is equivalent, by the above discussion, to a lifting

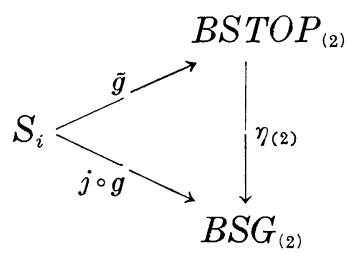


$\widetilde{g}$ is the unique lifting (up to equivalence) for which $l_{T O P} \circ T(\widetilde{f}): T(\rho) \rightarrow$ $M S T O P_{(2)} \rightarrow W S G_{(2)}$ is homotopic as a lifting to $\omega$. Applying the previous lemma again, we see that $\widetilde{l}_{T O P} \circ \widetilde{g}$ is homotopic to $g$ for exactly one homotopy class of maps $\tilde{g}: S^{i} \rightarrow B S T O P_{(2)}$. Thus $\widetilde{l}_{T O P^{*}}$ is an $\cong$ on $\pi_{i}$ for $i>1$. Since $X$ is the fiber of

$$
B S G_{(2)} \longrightarrow \prod_{i \geqq 1} K(Z / 2,4 i-1) \times K\left(Z_{(2)}, 4 i+1\right)
$$

the homotopy exact sequence of a fibration implies that $X$ is connected and simply connected as is $B S T O P(2)$. Thus $\widetilde{l}_{T O P}: B S T O P_{(2)} \rightarrow X$ is a homotopy equivalence.

7. The cohomology suspension of the classes $\tilde{\mathscr{C}}$ and $\tilde{\mathscr{L}}$. In $\S 5$ we defined classes $\tilde{\mathscr{\mathscr { K }}} \in H^{4^{*-1}}(B S G, \boldsymbol{Z} / 2)$ and $\tilde{\mathscr{L}} \in H^{4^{*}+1}\left(B S G, \boldsymbol{Z}_{(2)}\right)$ which are obstructions to transversality in the Thom space $M S G$. Our goal in this section is to study $\sigma(\tilde{\mathscr{K}}) \in H^{4^{*-2}}(S G, Z / 2)$ and $\sigma(\tilde{\mathscr{L}}) \in$ $H^{4^{*}}\left(S G, \boldsymbol{Z}_{(2)}\right)$, where the cohomology suspension $\sigma: H^{*}(B S G) \rightarrow H^{*-1}(S G)$ is induced by the natural map $\Sigma S G \rightarrow B S G$. We shall show that $\sigma(\tilde{\mathscr{K}})$ and $\sigma(\tilde{\mathscr{L}})$ are the classes induced from the surgery classes in $G / T O P$ by the natural map $G \rightarrow G / T O P$.

There is a fibration

$$
G_{q} / \text { TOP }_{q} \stackrel{j}{\longrightarrow} \operatorname{BSTOP}_{q} \longrightarrow B S G_{q}
$$

The bundle over $G_{q} / T O P_{q}$ is the universal topological $q$-bundle together with a fibre homotopy trivialization $t$, ( $t$ corresponds to a map of Thom spaces $\tau: M\left(G_{q} / T O P_{q}\right) \rightarrow S^{q}$ of degree one on the bottom cell). Moreover, the following diagram commutes

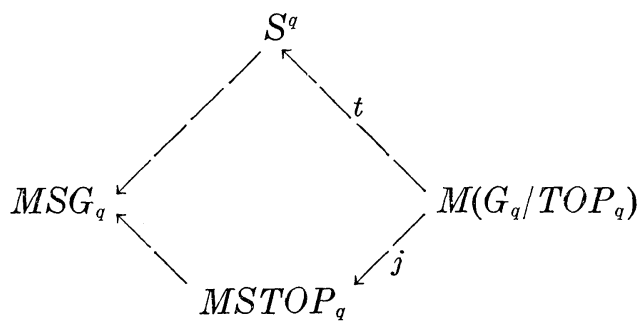

where the unnamed maps are the obvious maps of Thom spaces.

We have constructed natural fiber homotopy transversality structures on both $M S T O P_{q}$ and $S^{q}, q \geqq 3$; that is, liftings of $M S T O P_{q}$ and $S^{q}$ to $W S G_{q}$. This provides two liftings, $l_{\text {TOP }} \circ j$ and $l_{e} \circ t$ of $M\left(G_{q} / T O P_{q}\right)$ to $W S G_{q}$, as in the diagram follows: 


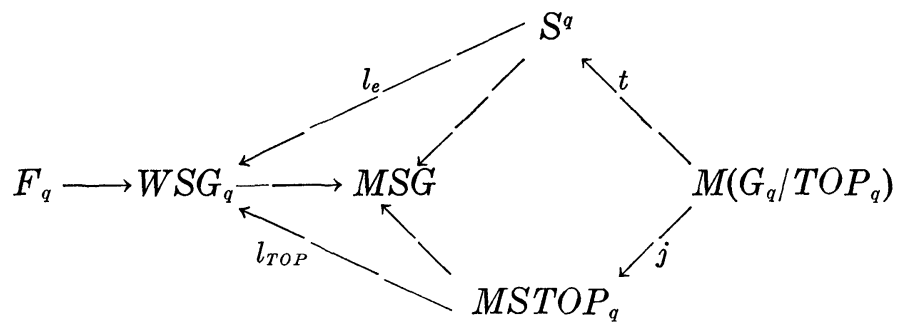

If we work in the stable range, or with spectra, we thus obtain a "difference element" $d: M(G / T O P) \rightarrow F, d=l_{T O P} \circ j-l_{e} \circ t$.

Using the homomorphisms of $\S 2, \mathcal{O}: \Omega_{*+q}\left(F_{q}\right) \rightarrow P_{*}$ and

$$
\mathcal{O}_{n}: \Omega_{*+q}\left(F_{q}, \boldsymbol{Z} / n\right) \longrightarrow P_{*} \otimes \boldsymbol{Z} / n,
$$

we constructed in $\S 5$ cohomology classes

$$
\mathscr{C} \times \mathscr{L}: F_{q} \longrightarrow \prod_{i \geqq 1} K(Z / 2, q+4 i-2) \times K\left(\boldsymbol{Z}_{(2)}, q+4 i\right) .
$$

Stably, we can form the composition $(\mathscr{K} \times \mathscr{L}) \circ d: M\left(G_{q} / T O P_{q}\right) \rightarrow$ $F_{q} \rightarrow \prod_{i \geqq 1} K(\boldsymbol{Z} / 2, q+4 i-2) \times K\left(\boldsymbol{Z}_{(2)}, q+4 i\right)$.

THEOREM 7.1. The map $(\mathscr{K} \times \mathscr{L}) \circ d$ coincides under the Thom isomorphism with the cohomology classes $\mathscr{K} \in H^{*^{*-2}}\left(G_{q} / T O P_{q}, \boldsymbol{Z} / 2\right)$ and $\mathscr{L} \in H^{4^{*}}\left(G_{q} /\right.$ TOP $\left._{q}, \boldsymbol{Z}_{(2)}\right)$, defined by the surgery obstruction homomorphisms $S: \Omega_{*}\left(G_{q} / T O P_{q}\right) \rightarrow P_{*}$ and $S_{n}: \Omega_{*}\left(G_{q} / T O P_{q}, Z / n\right) \rightarrow P_{*} \otimes Z / n$.

On the other hand, consider the fibrations

$$
S G \stackrel{\tilde{\mathscr{K}} \times \tilde{\mathscr{L}}}{\longrightarrow} \prod_{\imath \geqq 1} K(\boldsymbol{Z} / 2,4 i-2) \times K\left(\boldsymbol{Z}_{(2)}, 4 i\right) \longrightarrow X \longrightarrow B S G_{(2)}
$$

of $\S 6$, where

$$
X=\operatorname{Fiber}\left(\left(B S G_{(2)} \stackrel{\widetilde{\mathscr{K}} \times \widetilde{\mathscr{L}}}{\longrightarrow} \prod_{i \geqq 1} K(Z / 2,4 i-1) \times K\left(Z_{(2)}, 4 i+1\right)\right) .\right.
$$

By Theorem 6.2, the two lifts in the diagram

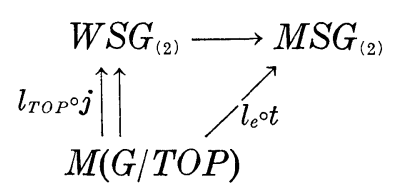

correspond to two lifts in the diagram

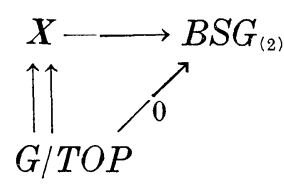


with a corresponding difference element $\Delta: G / T O P \rightarrow \prod_{i \geqq 1} K(Z / 2,4 i-2) \times$ $\left(Z_{(2)}, 4 i\right)$. But the lift of $0: G / T O P \rightarrow B S G_{(2)}$, corresponding to the lift $l_{e} \circ t$ on the Thom space, is the 0 lift, $0: G / T O P \rightarrow X$. From this remark and Theorem 7.1 it follows that the difference element $\Delta$ is $\mathscr{K} \times \mathscr{L}$ and that the following diagram commutes

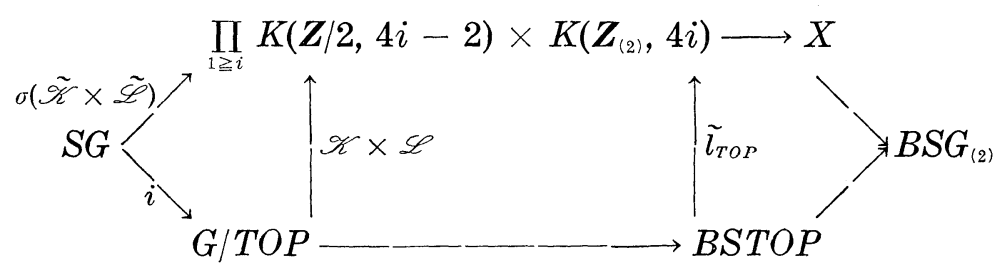

COROLLARY $7.2 . \quad \sigma(\tilde{\mathscr{L}})=i^{*}(\mathscr{\mathscr { L }}) \in H^{4^{*-2}}(S G, \boldsymbol{Z} / 2)$ and $\sigma(\tilde{\mathscr{L}})=$ $i^{*}(\mathscr{L}) \in H^{4^{*}}\left(S G, \boldsymbol{Z}_{(2)}\right)$.

Thus we see that the suspensions of the transversality obstructions $\tilde{\mathscr{C}}$ and $\tilde{\mathscr{L}}$ in $B S G$ are simply the surgery obstruction classes in $S G$. We now return to the proof of Theorem 7.1.

Proof of 7.1. For simplicity, we will prove 7.1 for $G / P L$ rather than G/TOP. This certainly suffices for Corollary 7.2.

The surgery obstruction homomorphisms $S: \Omega_{*}^{P L}\left(G_{q} / P L_{q}\right) \rightarrow P_{*}$ and $S_{n}: \Omega_{*}^{P L}\left(G_{q} / P L_{q}, Z / n\right) \rightarrow P_{*} \otimes Z / n$ have Thom space analogues $\mathscr{S}$ : $\Omega_{*+q}^{P L}\left(M\left(G_{q} / P L_{q}\right)\right) \rightarrow P_{*}$ and $\mathscr{S}_{n}: \Omega_{*,+q}^{P L}\left(L\left(G_{q} / P L_{q}\right), Z / n\right) \rightarrow P_{*} \otimes Z / n$ defined by composing $S$ and $S_{n}$ with the $P L$ bordism Thom isomorphism $\Omega_{*+q}^{P L}\left(M\left(G_{q} / P L_{q}\right)\right) \stackrel{\sim}{\longrightarrow} \Omega_{*}^{P L}\left(G_{q} / P L_{q}\right)$, and similarly with $\boldsymbol{Z} / n$ coeffecients. The cohomology calasses $\Phi(\mathscr{K}) \in H^{4^{*-2+q}}\left(M\left(G_{q} / P L_{q}\right), Z / 2\right)$ and

$$
\Phi(\mathscr{L}) H^{4^{*}+q}\left(M\left(G_{q} / P L_{q}\right), Z_{(2)}\right)
$$

are then defined in terms of $\mathscr{S}$ and $\mathscr{S}_{n}$ by formulae which are identical to the formulae defining $\mathscr{K} \in H^{4^{*-2+q}}\left(F_{q}, \boldsymbol{Z} / 2\right)$ and $\mathscr{L} \in$ $H^{4^{*+q}}\left(F_{q},\left(\boldsymbol{Z}_{(2)}\right)\right.$ in terms of $\mathcal{O}^{\circ}: \Omega_{*+q}\left(F_{q}\right) \rightarrow P_{*}$ and $\mathcal{O}_{n}: \Omega_{*+q}\left(F_{q}, \boldsymbol{Z} / n\right) \rightarrow$ $\boldsymbol{Z} / n$. (See $\S 5$ for these formulae.) Theorem 7.1 then follows from

$$
\mathscr{S}=\mathscr{O} d_{*}: \Omega_{*+q}\left(M\left(G_{q} / P L_{q}\right)\right) \longrightarrow \Omega_{*+q}\left(F_{q}\right) \longrightarrow P_{*} \text { and }
$$

$$
\mathscr{S}_{n}=\mathcal{O}_{n} d_{*}: \Omega_{*+q}\left(M\left(G_{q} / P L_{q}\right), \boldsymbol{Z} / n\right) \longrightarrow \Omega_{*+q}\left(F_{q}, \boldsymbol{Z} / n\right) \longrightarrow P_{*} \otimes \boldsymbol{Z} / n \text {. }
$$

To prove 7.3, we must examine the "difference construction" $d=l \circ j-l \circ t: M\left(G_{q} / P L_{q}\right) \rightarrow F_{q}$ more closely. Let us picture a cell $\alpha: \Delta^{q+i} \rightarrow M S G_{q}$ by: 
and a cell of $W S G_{q}$ by:

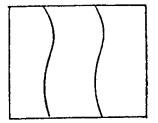

where the band is the $\mathrm{PD}$ space given by transversality. We picture a deformation $\Delta^{q+i} \times I \rightarrow M S G_{q}$ of $\alpha$ to $W S G_{q}$ by

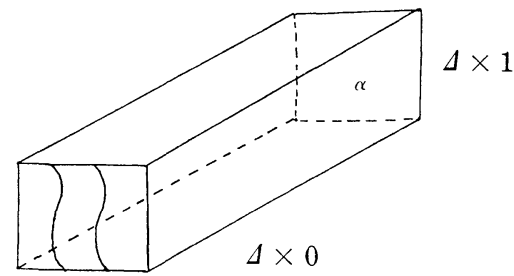

A cell of $F_{q}$ is pictured:

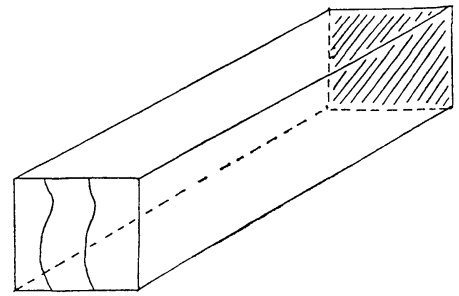

where the shaded top face means the map is to the basepoint of $M S G_{q}$. We will work in the stable range, $i \ll q$, and assume all our cells have a "suspension coordinate" in the direction of the arrow below:
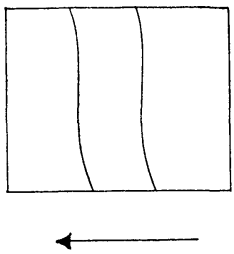

Cells of $F_{q}$ then "act" on deformations of $\alpha$ by suspension addition, or juxtaposition: 

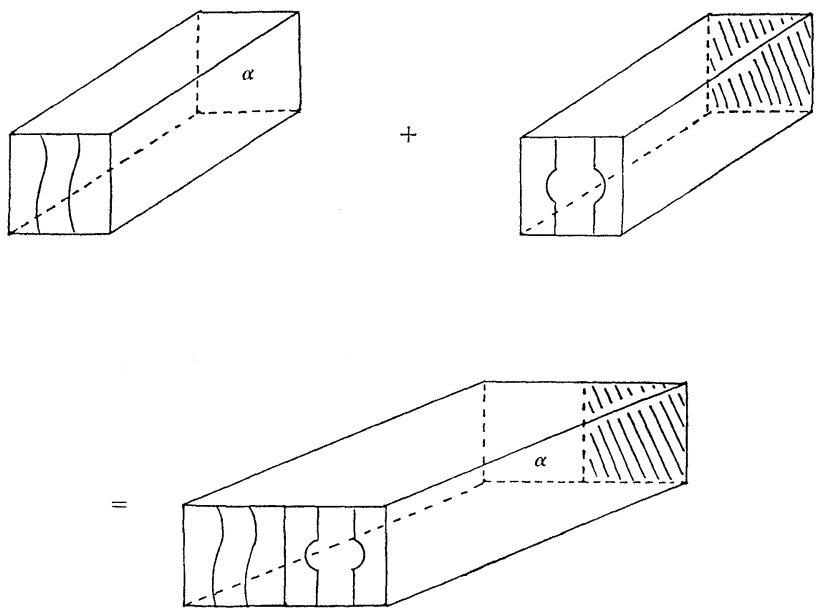

followed by the obvious equivalence of

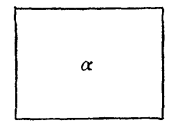

and

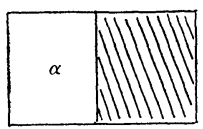

With these definitions one shows for complexes $X$ with $\operatorname{dim}(X) \ll 2 q$ that if $f: X \rightarrow M S G_{q}$ can be deformed to $W S G_{q}$, then equivalence classes of deformations of $f$ correspond bijectively with $\left[X, F_{q}\right]$.

Now consider a cell $\alpha: \Delta^{q+i} \rightarrow M\left(G_{q} / P L_{q}\right)$. We picture the deformation $l_{P L^{\circ}} j(\alpha)$ defined by $P L$ transversality by:

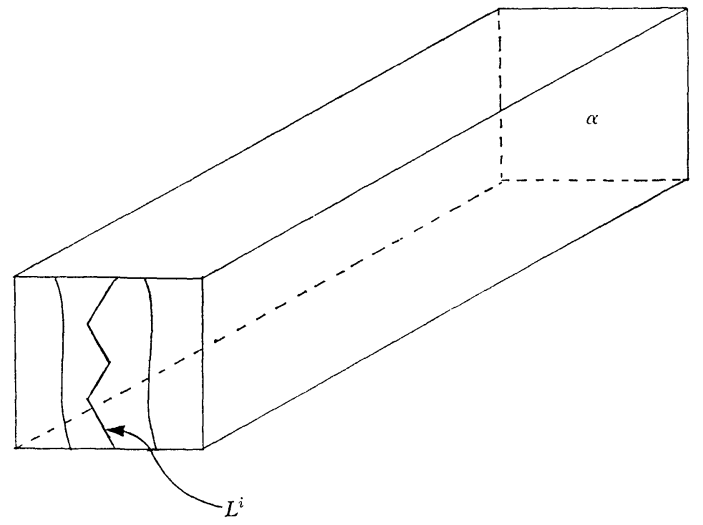

where $L^{i}$, the core of the band on the bottom face, is a $P L$ manifold and the band is a tubular neighborhood of $L^{i}$. We picture the deformation $l_{e} \circ t(\alpha)$ defined by the fibre homotopy trivialization of $M\left(G_{q} / P L_{q}\right)$ followed by transversality for the trivial bundle, 


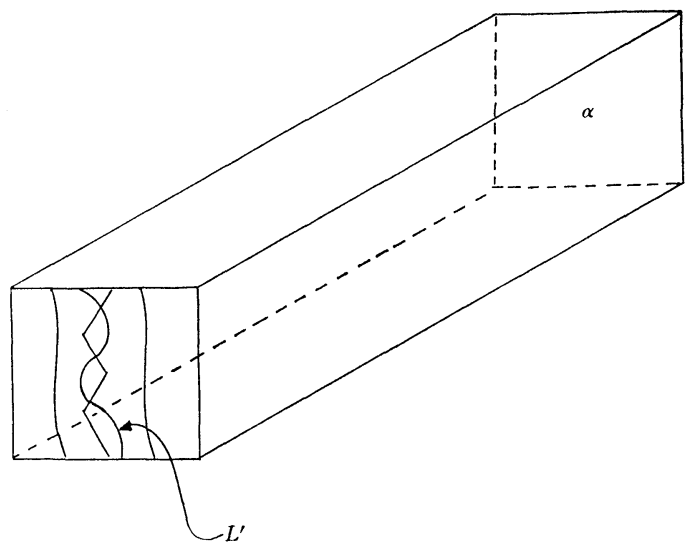

where $L^{\prime}$ is a framed submanifold of the tubular neighborhood of $L$. The following picture gives a cell, $d(\alpha)$, of $F_{q}$ :

$(7.4)$

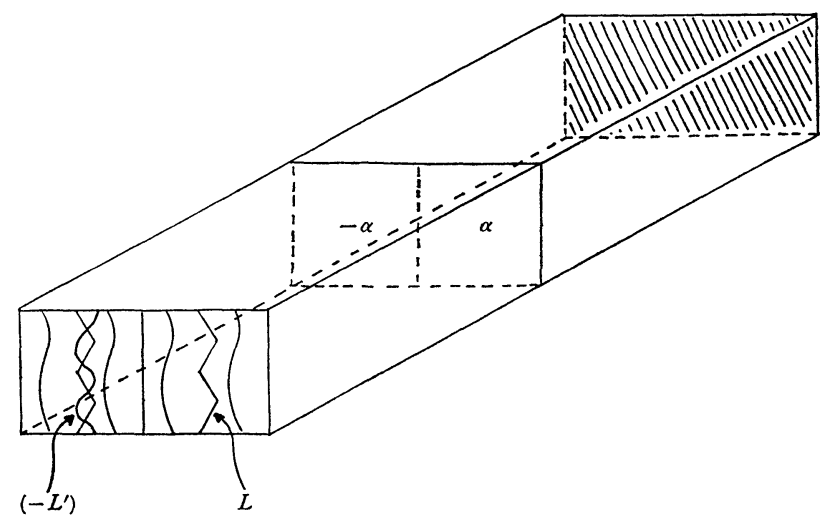

where the map on the top half is a canonical homotopy of $\alpha-\alpha$ to the trivial map. It is easy to see that the deformation $l_{e} \circ t(\alpha)+d(\alpha)$ is equivalent to $l_{P L} \circ j(\alpha)$. Thus $d(\alpha)$ represents the difference construction $l_{P L} \circ j(\alpha)-l_{e} \circ t(\alpha): \Delta^{q+i} \rightarrow F_{q}$.

If we have, instead of a single cell, a manifold $f: M^{q+i} \rightarrow M\left(G_{q} / P L_{q}\right)$, we get $d(f): M \rightarrow F_{q}$ by iterating the construction above over the cells of $M$. This produces a $P L$ submanifold $L^{i} \subset M^{q+i}$ and a framed submanifold $L^{\prime}$ of the tubular neighborhood $V_{L \subset M}$. It is easy to see from the definition of $\mathscr{S}: \Omega_{*+q}\left(M\left(G_{q} / P L_{q}\right)\right) \rightarrow P_{*}$ above that

$$
\mathscr{S}([M, f])=S\left(L^{\prime} \rightarrow L\right) \in P_{i},
$$

and similarly for $\mathscr{S}_{n}$ if $M$, and hence $L^{\prime}$ and $L$, are $\boldsymbol{Z} / n$ manifolds.

On the other hand, from $\S 2, \mathcal{O}([M, d(f)])$ is defined as the obstruction to making the cells of $d(f)$ pictured in 7.4 globally transversal, keeping the bottom face fixed. We may as well assume that 
$f: M \rightarrow M\left(G_{q} / P L_{q}\right)$ is already $P L$ transversal, so that 7.4 simplifies to

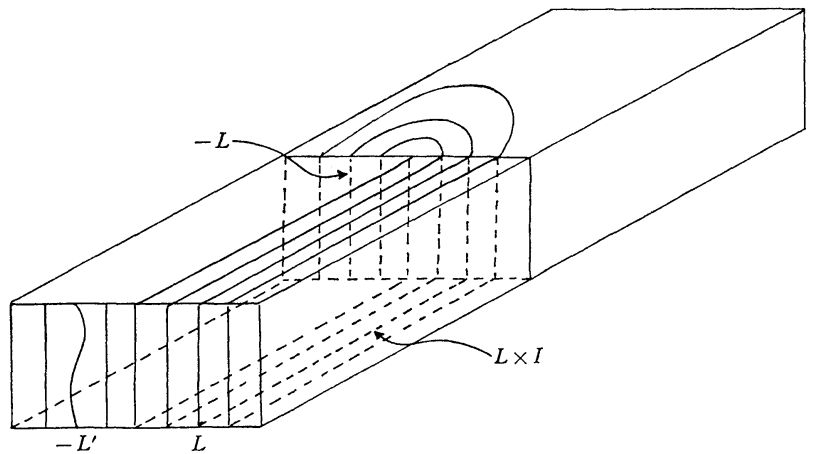

That is, $d(f)$ is already transversal except in the lower left block, and in the lower left block, $d(f)$ is the homotopy between the Pontrjagin-Thom constructions on the $P L$ normal bundle of $L$ and the framed normal bundle of $L^{\prime}$. Thus

$\mathcal{O}([M, d(f)])=S\left(L^{\prime} \rightarrow L\right)=\mathscr{S}([M, F]) \in P_{i}$ and similarly for $\mathscr{O}_{n}$. This proves 7.3 and completes the proof of Theorem 7.1 .

REMARK 7.5. If one follows to the letter the prescription of $\S 2$ for computing $\mathscr{O}\left([M, d(f)] \in P_{i}\right.$, one acutally gets $\mathscr{O}([M, d(f)])=$ $S\left(L^{\prime} \cup(-L) \rightarrow L \cup(-L)\right)$. Of course, this is the same as $S\left(L^{\prime} \rightarrow L\right)$.

REMARK 7.6. We leave for the reader the details of the argument for G/TOP. It is only necessary to carry through the "crossing with $C P(2)$ " construction used in the definition of $l_{T O P}: M S T O P_{q} \rightarrow$ $W S G_{q}$.

REMARK 7.7. The results 7.2 and 6.2 suggest that the map $\tilde{\mathscr{K}} \times$ $\tilde{\mathscr{L}}: B S G \rightarrow \prod_{n \geqq 1} K(\boldsymbol{Z} / 2,4 n-1) \times K\left(\boldsymbol{Z}_{(2)}, 4 n+1\right)$ is in some sense equivalent to the natural map $B \pi: B S G \rightarrow B(G / T O P)_{(2)}$. That is, we have identified the fibre of $\tilde{\mathscr{K}} \times \tilde{\mathscr{L}}$ with $B S T O P_{(2)}$ (Theorem 6.2) and we have identified $\Omega(\check{\mathscr{K}} \times \tilde{\mathscr{L}}): S G \rightarrow \Pi K(\boldsymbol{Z} / 2,4 n-2) \times K\left(\boldsymbol{Z}_{(2)}, 4 n\right)$ with $S G \rightarrow$ $(G / T O P)_{(2)}$. I. Madsen and R. J. Milgram have proved that $B(G / T O P)_{(2)}$ is a product of Eilenberg-MacLane spaces, but they have also shown that there does not exist a commutative diagram

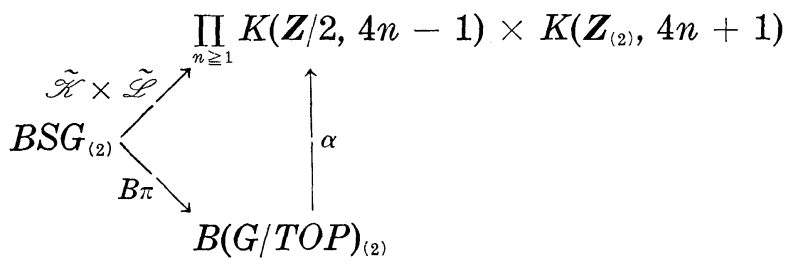


with $\alpha$ a homotopy equivalence.

\section{CHAPTER III}

8. A Hirzebruch index class for PD spaces. In previous sections, we have defined cohomology classes $\tilde{\mathscr{K}} \in H^{4^{*-1}}(B S G, Z / 2)$ and $\tilde{\mathscr{L}} \in H^{4^{*}+1}\left(B S G, \boldsymbol{Z}_{(2)}\right)$ which are obstructions to Poincaré transversality in the Thom space $M S G$. From Lemma 3.7, it follows trivially that $8 \tilde{\mathscr{L}}=0$. Thus, there exist classes $l \in H^{4 *}(B S G, Z / 8)$ such that $\beta l=\tilde{\mathscr{L}}$, where $\beta$ is the Bockstein homomorphism of the coefficient sequence $0 \rightarrow \boldsymbol{Z}_{(2)} \rightarrow \boldsymbol{Z}_{(2)} \rightarrow \boldsymbol{Z} / 8 \rightarrow 0$.

In this chapter, we will define a specific class $l=1+l_{1}+l_{2}+\cdots \epsilon$ $H^{*^{*}}(B S G, \boldsymbol{Z} / 8)$ with $\beta l=\tilde{\mathscr{L}}$. Our class $l$ is a kind of Hirzebruch class, which measures the index modulo 8 for PD spaces. Among its properties are the following:

8.1. (i) If $M^{4 n}$ is a $Z / 8$ PD space and $\nu: M^{4 n} \rightarrow B S G$ classifies the stable fibration of $M^{4 n}$, then

$$
\left\langle\nu^{*}(l),\left[M^{4 n}\right]\right\rangle=\operatorname{index}\left(M^{4 n}\right) \in \boldsymbol{Z} / 8 .
$$

(ii) $\rho_{2}(l)=V^{2} \in H^{4^{*}}(B S G, Z / 2)$.

(iii) $l$ satisfies the Whitney sum formula $l(\xi \times \eta)=l(\xi) \otimes l(\eta)+$ $i\left(V S q^{1} V(\xi) \otimes \tilde{\mathscr{K}}(\eta)+\tilde{\mathscr{K}}(\xi) \otimes V S q^{1} V(\eta)\right), \quad$ where $\quad i: H^{*}(, \boldsymbol{Z} / 2) \rightarrow$ $H^{*}(, \boldsymbol{Z} / 8)$ is induced by the inclusion $i: \boldsymbol{Z} / 2 \rightarrow \boldsymbol{Z} / 8$.

(iv) $\beta l=\tilde{\mathscr{L}} \in H^{4^{*+1}}\left(B S G, \boldsymbol{Z}_{(2)}\right)$.

(v) $\pi^{*}(l)=\rho_{8}(L) \in H^{4^{*}}(B S T O P, Z / 8)$, where $\pi: B S T O P \rightarrow B S G$ is the natural map and $L \in H^{4^{*}}\left(B S T O P, \boldsymbol{Z}_{(2)}\right)$ is the topological $L$-class of [15].

(vi) $\rho_{4}(l) \in H^{4^{*}}(B S G, \boldsymbol{Z} / 4)$ is the $\boldsymbol{Z} / 4$ index class constructed in [6]. In particular, $\rho_{4}\left(l_{n}\right)=\mathscr{P}\left(v_{2 n}\right)+j \sigma_{n}\left(w_{2} w_{3}, \cdots, w_{4 n}\right)$, where $\mathscr{P}$ is the Pontrjagin square, $j: H^{*}(B S G, Z / 2) \rightarrow H^{*}(B S G, Z / 4)$ is the natural map, and $\sigma_{n}$ is a polynomial in Stiefel-Whitney classes.

It is not difficult to show that properties 8.1(i), (ii), and (iii) characterize $l$ uniquely. The existence of a $Z / 8$ class satisfying 8.1 (i) was shown by D. Frank, using the same theory of N. Levitt that we have exploited in this paper. The existence of a $Z / 8$ class satisfying 8.1(ii) has been known for some time, as it is easy to compute homotopy-theoretically the obstruction to lifting a square to a $\boldsymbol{Z} / 8$ class. It is surprisingly difficult, however, to construct a single class which satisfies 8.1(i), (ii), and (iii). Property (iii) is particularly interesting for historical reasons, and by analogy with other index classes (the $\boldsymbol{Z}_{(2)}$-class of [15] and the $\boldsymbol{Z} / 4$ class of [6]). One might 
expect the class $l$ to satisfy the sum formula $l(\xi \times \eta)=l(\xi) \otimes l(\eta)$. However, we can prove there is no class which satisfies this simpler formula and which also satisfies 8.1(i).

To construct $l \in H^{4^{*}}(B S G, Z / 8)$, we will define a suitable homomorphism on the smooth bordism of $M S G, \varphi: \Omega_{4^{*}}(M S G, Z / 8) \rightarrow Z / 8$, and use Proposition A.11 of the Appendix to obtain $\Phi(l) \in H^{4^{*}}(M S G, Z / 8)$, where $\Phi: H^{4^{*}}(B S G, Z / 8) \simeq H^{4^{*}}(M S G, Z / 8)$ is the Thom isomorphism.

Let

$$
\Omega_{4^{*}}^{\prime}(M S G, Z / 8)=\operatorname{kernel}\left(\Omega_{4^{*}}(M S G, Z / 8) \stackrel{\delta}{\longrightarrow} \Omega_{4^{*}-1}(M S G) \stackrel{s}{\longrightarrow} Z / 2\right) .
$$

We first define $\varphi^{\prime}: \Omega_{4^{*}}^{\prime}(M S G, Z / 8) \rightarrow Z / 8$. Roughly, given $f: M^{q+4 n} \rightarrow$ $M S G_{q}$ with $q \gg 4 n, M$ a $Z / 8$ manifold, and $s\left(\left[\delta M,\left.f\right|_{\left.{ }_{\partial M}\right]}\right]\right)=0$, we use Theorem 3.4 to cobord $f: M^{q+4 n} \rightarrow M S G_{q}$ to a globally transversal map $g: N^{q+4 n} \rightarrow M S G_{q}$. Let $g^{-1}\left(B S G_{q}\right)=L^{4 n} \subset N^{q+4 n}$ be the associated $\boldsymbol{Z} / 8$ PD space. Set $\varphi^{\prime}[M, f]=\operatorname{index}\left(L^{4 n}\right) \in \boldsymbol{Z} / 8$. It is not obvious that $\varphi^{\prime}$ is well-defined, since a globally transversal map $g: N^{q+4 n} \rightarrow$ $M S G_{q}$ may be cobordant to 0 , but not "globally transversally cobordant to $0 . "$ We need a generalization of Lemma 3.6.

Lemma 8.2. If $N^{q+4 n}$ is a $Z / 8$ manifold, $g: N^{q+4 n} \rightarrow M S G_{q}$ a globally transversal map, with $K^{4 n}=g^{-1}\left(B S G_{q}\right)$, and if $[N, g]=0 \in \Omega_{q+4 n}\left(M S G_{q}\right.$, $\boldsymbol{Z} / 8)$, then index $\left(K^{4 n}\right) \equiv 0(\bmod 8)$.

Proof. The proof is analogous to the proof of Lemma 3.6. There is an isomorphism $p: \widetilde{\Omega}_{q+j}\left(M S G_{q}, Z / 8\right) \stackrel{\sim}{\rightarrow} \pi_{3 q+j}\left(M S G_{q} \wedge M S O_{2 q}, Z / 8\right)$ if $j<q-2$. Elements of $\pi_{3 q+j}\left(M S G_{q} \wedge M S O_{2 q}, Z / 8\right)$ are represented by maps $S_{8}^{3 q+j} \rightarrow M S G_{q} \wedge M S O_{2 q}$, where $S_{8}^{3 q+j}$ is the space obtained from $S^{3 q+j}$ by deleting 8 discs, and then identifying the boundary components. $\quad S_{8}^{3 q+j}$ is a $\boldsymbol{Z} / 8$ manifold, and every $\boldsymbol{Z} / 8$ manifold $N^{q+j}$ embeds in $S_{8}^{3 q+j}$ (as $\boldsymbol{Z} / 8$ manifolds) with tubular neighborhood the stable normal bundle of $N^{q+j}$. Such an embedding defines $p([N, g]) \in$ $\pi_{3 q+j}\left(M S G_{q} \wedge M S O_{2 q}, Z / 8\right)$. In particular, if $g: N^{q+4 n} \rightarrow M S G_{q}$ is globally transversal, $\alpha=p([N, g]): S_{8}^{3 q+4 n} \rightarrow M S G_{q} \wedge M S O_{2 q}$ is globally transversal; in fact, $g^{-1}\left(B S G_{q}\right)=K^{4 n}$ and $\alpha^{-1}\left(B S G_{q} \times B S O_{2 q}\right)=X^{4 n}$ are homotopy equivalent $Z / 8$ PD spaces. Thus index $\left(X^{4 n}\right)=\operatorname{index}\left(K^{4 n}\right)$.

Careful use of the obstruction theory of $\S 1$, Chapter I (in particular, Theorem 1.3(3) and Lemma 1.4) implies that we may homotope $\alpha: S_{8}^{3 q+4 n} \rightarrow M S G_{q} \wedge M S O_{2 q}$ to a strongly transversal map $\beta$, with $\beta^{-1}\left(B S G_{q} \times B S O_{2 q}\right)=Y^{4 n} \subset X^{4 n}$, a degree one normal map of $\boldsymbol{Z} / 8$ manifolds, which is homotopy equivalence on the Bockstein $\delta Y \subset \delta X$. Thus index $\left(Y^{4 n}\right) \equiv \operatorname{index}\left(X^{4 n}\right)(\bmod 8)$.

On the other hand, another application of the obstruction theory implies that the $\boldsymbol{Z} / 8 \mathrm{PD}$ space $Y^{4 n}$, obtained from the strongly 
transversal map $\beta$, is determined by homotopy class of $\beta: S_{8}^{3 q+4 n} \rightarrow$ $M S G_{q} \wedge M S O_{2 q}$, modulo cobordism of $Z / 8 \mathrm{PD}$ spaces and connected sum with the Milnor manifold $M^{4 n}$ of index 8 . The lemma follows.

Now, $s \delta: \Omega_{4 n}(M S G, Z / 8) \rightarrow Z / 2$ is nonzero for all $n>0$. In fact, let $K^{q+4}=S^{q+3} \times I /(x, 0) \equiv(-x, 1)$ be the $q+4$-dimensional Klein bottle. $K^{q+4}$ is a $Z / 2$ manifold with $\delta K^{q+4}=S^{q+3}$. In Chapter IV, $\S 10$, we will show that there exists a map $\alpha: K^{q+4} \rightarrow M S G_{q}$, with:

$$
\text { (i ) } s \delta\left[K^{q+4}, \alpha\right]=s\left[S^{q+3},\left.\alpha\right|_{S^{q+3}}\right]=1 \in Z / 2 \text { and }
$$

(ii) $\left\langle\alpha^{*}\left(\Phi\left(V_{2}^{2}\right)\right),\left[K^{q+4}\right]\right\rangle=0 \in Z / 2$.

By Lemma 3.1(iii), $s \delta\left(\left[K^{q+4}, \alpha\right][C P(2(n-1))]\right)=1 \in Z / 2$. Thus, on $\Omega_{q+4 n}\left(M S G_{q}, Z / 8\right), \quad s \delta$ is nonzero on $i\left[K^{q+4}, \alpha\right][C P(2(n-1))]$, where $i: \Omega_{*}(, \boldsymbol{Z} / 2) \rightarrow \Omega_{*}(, \boldsymbol{Z} / 8)$.

Define $r: \Omega_{q+4 n}(M S G, Z / 8) \rightarrow \Omega_{q+4 n}^{\prime}(M S G, Z / 8)$ by

$$
r([M, f])=[M, f]-i\left((s \delta[M, f])\left[K^{q+4}, \alpha\right][C P(2(n-1))]\right) .
$$

where the coefficient $(s \delta[M, f])$ is 0 or 1 . As the difference of two homomorphisms, $r$ is certainly a homomorphism. We then define

$$
\varphi^{\prime \prime}=\varphi^{\prime} \circ r: \Omega_{4^{*}}(M S G, \boldsymbol{Z} / 8) \longrightarrow \boldsymbol{Z} / 8 \text {. }
$$

It turns out that $\varphi^{\prime \prime}$ does not quite satisfy the product formulae of Proposition A.11. We need a correction term on products of the form $\left[P^{q+4 a-1}, g\right]\left[Q^{4 b+1}\right] \in \Omega_{q+4(a+b)}\left(M S G^{q}, Z / 8\right)$. So we finally define $\varphi: \Omega_{4^{*}}(M S G, \boldsymbol{Z} / 8) \rightarrow \boldsymbol{Z} / 8$ by

$$
\varphi[M, f]=\varphi^{\prime \prime}[M, f]-i\left\langle V S q^{1} V(M) \cdot f^{*}(\tilde{\mathscr{K}}),[M]\right\rangle \in \boldsymbol{Z} / 8,
$$

where $\tilde{\mathscr{K}} \in H^{4^{*-1}}(B S G, \boldsymbol{Z} / 2)$ is the class of $\S 5$ and $i: \boldsymbol{Z} / 2 \rightarrow \boldsymbol{Z} / 8$ is the inclusion. In dimensions $\not \equiv 0(\bmod 4)$, we set $\varphi \equiv 0$.

THEOREM 8.4. (i) $\varphi([M, f][N])=\varphi[M, f] \cdot \operatorname{index}(N) \in \boldsymbol{Z} / 8$ if $[M, f] \in \Omega_{*}(M S G, Z / 8)$ and $[N] \in \Omega_{*}(p t)$.

(ii) $\varphi(i[\tilde{M}, \widetilde{f}][\tilde{N}])=\varphi(i[\widetilde{M}, \widetilde{f}]) \cdot i n d e x \quad(\widetilde{N}) \in \boldsymbol{Z} / 2 \subset \boldsymbol{Z} / 8$ if $[\tilde{M}, \widetilde{f}] \epsilon$ $\Omega_{*}(M S G, \boldsymbol{Z} / 2)$ and $[\tilde{N}] \in \Omega_{*}(p t, \boldsymbol{Z} / 2)$.

(iii) $\varphi\left(\rho_{8} \delta([\widetilde{P}, \widetilde{F}][\widetilde{Q}])\right)=\varphi\left(\rho_{8} \delta[\widetilde{P}, \widetilde{F}]\right) \cdot i n d e x(\widetilde{Q}) \in \boldsymbol{Z} / 2 \subset \boldsymbol{Z} / 8$ if $[\widetilde{P}, \widetilde{F}] \epsilon$ $\Omega_{*}(M S G, Z / 2)$ and $[\widetilde{Q}] \in \Omega_{*}(p t, Z / 2)$.

Before proving 8.4 , we establish the main results of this section. From 8.4 and Proposition A.11, of the Appendix, we have

Theorem 8.5. There is a unique class $l=1+l_{1}+l_{2}+, \cdots, l_{i} \in$ $H^{4 i}(B S G, \boldsymbol{Z} / 8)$, such that, for any map $f: M \rightarrow M S G, M$ a smooth $\boldsymbol{Z} / 8$ manifold, 


$$
\begin{aligned}
& \varphi^{\prime}(r[M, f]) \\
& \quad=\left\langle L(M) \cdot f^{*}(\Phi(l)),[M]\right\rangle+i\left\langle V S q^{1} V(M) \cdot f^{*}(\tilde{\mathscr{K}}),[M]\right\rangle \in \boldsymbol{Z} / 8 .
\end{aligned}
$$

We next establish properties 8.1(i), (ii), and (iv) for the class $l$.

Proof of 8.1. (i) Let $S_{8}^{q+4 n}$ be the $Z / 8$ manifald defined in the proof of Lemma 8.2. If $M^{4 n}$ is a $Z / 8$ PD space, $q \gg 4 n$, we may embed $M^{4 n} \subset S_{8}^{q+4 n}$ as $Z / 8$ PD spaces, so that the normal bundle $M^{4 n}$ in $S_{8}^{q+4 n}$ is the stable normal fibration $\nu$ of $M^{4 n}$. The Prontrjagin-Thom construction then gives a globally transversal $\boldsymbol{Z} / 8$ bordism element, $p: S_{8}^{q+4 n} \rightarrow M S G_{q}$, with $p^{-1}\left(B S G_{q}\right)=M^{4 n}$ and $\left.p\right|_{M}=\nu: M \rightarrow B S G_{q}$. Then, by the definitions of $\varphi^{\prime}$ and $r$, and by 8.5 ,

$$
\begin{aligned}
\operatorname{index}\left(M^{4 n}\right) & =\varphi^{\prime}\left[S_{8}^{q+4 n}, P\right] \\
& =\varphi^{\prime} r\left[S_{8}^{q+4 n}, P\right] \\
& =\left\langle p^{*}(\Phi(l)),\left[S_{8}^{q+4 n}\right]\right\rangle \\
& =\left\langle\left(\left.p\right|_{M}\right)^{*}(l),\left[M^{4 n}\right]\right\rangle \\
& =\left\langle\nu^{*}(l),\left[M^{4 n}\right]\right\rangle \in \boldsymbol{Z} / 8,
\end{aligned}
$$

since $L\left(S_{8}^{q+4 n}\right)=1$ and $V S q^{1} V\left(S_{8}^{q+4 n}\right)=0$.

Proof of 8.1. (ii) Let $[N, g] \in \Omega_{4^{*}}(M S G, Z / 2)$. We will prove, without using 8.4, that

$$
\varphi i([N, g])=i\left\langle L(N) \cdot g^{*} \Phi\left(V^{2}\right),[N] \in \boldsymbol{Z} / 2 \subset \boldsymbol{Z} / 8 .\right.
$$

On the other hand, it is obvious from 8.5 that

$$
\text { ๑ } i([N, g])=i\left\langle L(N) \cdot g^{*} \Phi\left(\rho_{2} l\right),[N]\right\rangle \in \boldsymbol{Z} / 2 \subset \boldsymbol{Z} / 8 \text {. }
$$

(That is to say, in the language of Proposition A.11, or A.3, the graded class $\Phi\left(\rho_{2}(l)\right) \in H^{*}(M S G, \boldsymbol{Z} / 2)$ corresponds to the homomorphism $\varphi i$ : $\Omega_{*}(M S G, \boldsymbol{Z} / 2) \rightarrow \boldsymbol{Z} / 2 \subset \boldsymbol{Z} / 8$.) It follows immediately that $\rho_{2} l=V^{2}$.

To prove 8.6, let $\Omega_{4 *}^{\prime}(M S G, Z / 2)=\operatorname{kernel}\left(s \delta: \Omega_{4^{*}}(M S G, Z / 2) \rightarrow\right.$ $\left.\Omega_{4^{*}-1}(M S G) \rightarrow Z / 2\right)$. There is a commutative diagram

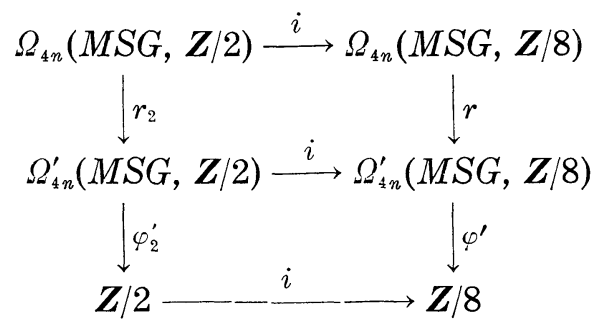

where $r_{2}\left[M^{q+4 n}, f\right]=\left[M^{q+4 n}, f\right]-(s \delta[M, f])\left[K^{q+4}, \alpha\right][C P(2 n-2)]$, and where, if $g: N^{q+4 n} \rightarrow M S G_{q}$ is a globally transversal $Z / 2$ manifold, 
$\varphi_{2}^{\prime}[N, g]=\operatorname{index}\left(g^{-1}\left(B S G_{q}\right)\right) \in \boldsymbol{Z} / 2$. (The fact $\varphi_{4 n}^{\prime}(M S G, \boldsymbol{Z} / 8) \rightarrow \boldsymbol{Z} / 8$ is well-defined implies $\varphi_{2}^{\prime}: \Omega_{4 n}^{\prime}(M S G, \boldsymbol{Z} / 2) \rightarrow \boldsymbol{Z} / 2$ is well-defined.) From wellknown properties of $V^{2}$ and $L$ (namely: (a) $V^{2}$ is multiplicative, (b) $\left\langle\nu^{*}\left(V^{2}\right),\left[M^{4 n}\right]\right\rangle=$ index $\left(M^{4 n}\right) \in Z / 2$ if $\nu: M^{4 n} \rightarrow B S G$ is the normal bundle of a $Z / 2 \mathrm{PD}$ space $M^{4 n}$, and (c) $\rho_{2}(L(N))=V^{2}(N)$ for smooth manifolds $N$ ), it is easy to see that

$$
\begin{aligned}
\varphi_{2}^{\prime}[N, g] & =\operatorname{index}\left(g^{-1}\left(B S G_{q}\right)\right) \\
& =\left\langle V^{2}(N) \cdot g^{*} \Phi\left(V^{2}\right),[N]\right\rangle \\
& =\left\langle L(N) \cdot g^{*} \Phi\left(V^{2}\right),[N]\right\rangle \in \boldsymbol{Z} / 2 .
\end{aligned}
$$

If $f: M^{q+4 n} \rightarrow M S G_{p}$ is any $Z / 2$ bordism element, we then have

$$
\begin{aligned}
\varphi_{2}^{\prime} r_{2}[M, f]=\varphi_{2}^{\prime}\left([M, f]-(s \delta[M, f])\left[K^{q+4}, \alpha\right][C P(2 n-2)]\right) \\
=\left\langle L(M) f^{*} \Phi\left(V^{2}\right),[M]\right\rangle-(s \delta[M, f])\left\langle L\left(K^{q+4} \times C P(2 n-2)\right)\right. \\
\\
\left.\cdot\left(\alpha^{*}\left(\Phi\left(V^{2}\right)\right) \otimes 1\right),\left[K^{q+4} \times C P(2 n-2)\right]\right\rangle \\
=L\left\langle(M) \cdot f^{*} \Phi\left(V^{2}\right),[M]\right\rangle .
\end{aligned}
$$

The first equality is the definition of $r_{2}$, the second equality is 8.7 , and the third equality follows from 8.3(ii) and the fact that $L\left(K^{q+4}\right)=1$.

Finally, it is obvious that on elements $[M, f] \in i\left(\varphi_{4^{*}}(M S G, Z / 2)\right) \subset$ $\Omega_{4^{*}}(M S G, \boldsymbol{Z} / 8)$, the error term in the definition of $\varphi$ vanishes; that is, $i\left\langle V S q^{1} V(M) \cdot f^{*}(\tilde{\mathscr{C}}),[M]\right\rangle=0$. Thus we have proved 8.6:

$$
\begin{aligned}
\varphi_{i}[N, g] & =\varphi^{\prime} \operatorname{ri}[N, g]=i \varphi_{2}^{\prime} r_{2}[N, g] \\
& =i\left\langle L(N) \cdot g^{*} \Phi\left(V^{2}\right),[N] \in \boldsymbol{Z} / 2 \subset \boldsymbol{Z} / 8 .\right.
\end{aligned}
$$

Proof of 8.1. (iv) We will prove, without using 8.4, that the homomorphisms

$$
\Omega_{4^{*}+1}\left(M S G, \boldsymbol{Z} / 2^{s}\right) \stackrel{\delta}{\longrightarrow} \Omega_{4^{*}}(M S G) \stackrel{\varphi \rho_{8}}{\longrightarrow} \boldsymbol{Z} / 8,
$$

with image interpreted in $\boldsymbol{Z} / 2^{s}, s \geqq 1$, are exactly the defining homomorphisms for the class $\Phi(\tilde{\mathscr{L}}) \in H^{4^{*+1}}\left(M S G, \boldsymbol{Z}_{(2)}\right)$ (see the Appendix, A.6 and A.7); that is, if $[M, f] \in \Omega_{*}\left(M S G, Z / 2^{s}\right)$, we will prove that

$$
\varphi \rho_{8} \delta[M, f]=s_{2^{s}}([M, f])-i\left\langle V S q^{1} V(\delta M) \cdot\left(\left.f\right|_{{ }_{\delta M}}\right) *\left(\tilde{\check{\varkappa}^{\prime}}\right),[\delta M]\right\rangle \in \boldsymbol{Z} / 2^{s},
$$

where $i: \boldsymbol{Z} / 2 \rightarrow \boldsymbol{Z} / 2^{s}$. It follows immediately, as in A.13 of the Appen$\operatorname{dix}$, that $\beta l=\mathscr{L}$.

We obviously have $\rho_{8} \Omega_{4^{*}}(M S G) \subset \Omega_{4^{*}}^{\prime}(M S G, Z / 8)$, because $\delta \rho_{8} \equiv 0$, hence $s \delta \rho_{8} \equiv 0$. Thus $\phi^{\prime \prime} \rho_{8}=\varphi^{\prime} \rho_{8}: \Omega_{4^{*}}(M S G) \rightarrow Z / 8$, hence $\varphi \rho_{8} \delta$ : $\Omega_{4 n+1}\left(M S G, \boldsymbol{Z} / 2^{s}\right) \rightarrow \boldsymbol{Z} / 8$ is computed as follows. Given $f: M^{++4 n+1} \rightarrow$ $M S G_{q}$, a $Z / 2^{s}$ bordism element, assume (after a cobordism) that $\left.f\right|_{\delta M}$ is globally transversal with $f^{-1}\left(B S G_{q}\right)=L^{4 n} \subset \delta M$. Then 


$$
\varphi \rho_{8} \delta[M, f]=\operatorname{index}\left(L^{4 n}\right)-i\left\langle V S q^{1} V(\delta M) \cdot\left(\left.f\right|_{\delta M}\right)^{*}(\tilde{\mathscr{K}}),[\delta M]\right\rangle \in \boldsymbol{Z} / 8 .
$$

Of course, we interpret this number in $\boldsymbol{Z} / 2^{s}$. By Chapter I, Lemma 3.7, and 5.4(ii) of Chapter II, this exactly the homomorphism

$$
\Omega_{4^{*}+1}\left(M S G, Z / 2^{s}\right) \longrightarrow Z / 2^{s}
$$

which defines $\Phi(\tilde{\mathscr{L}}) \in H^{4^{*}+1}\left(M S G, Z_{(2)}\right)$, as desired.

The proof of 8.1(iii) is rather difficult, and will be discussed in $\S 9$. The property $8.1(\mathrm{v})$ of our class $l$ follows easily from $8.1(\mathrm{i})$, (ii), and (iii), and the the discussion in [15] where characterizing properties of $\rho_{8}(L) \in H^{4 *}(B S T O P, \boldsymbol{Z} / 8)$ are listed.

Proof of 8.1. (vi) From the definition of $\varphi: \Omega_{4^{*}}(M S G, \boldsymbol{Z} / 8) \rightarrow Z / 8$ and Theorem 8.5, we see that, if $f: M^{q+4 n} \rightarrow M S G_{q}$ is a globally transversal $\boldsymbol{Z} / 4$ manifold, with $L^{4 n}=f^{-1}\left(B S G_{q}\right) \subset M^{q+4 n}$, then

$$
\text { index }\left(L^{4 n}\right)=\left\langle L(M) \cdot f^{*} \Phi\left(\rho_{4}(l)\right),[M]\right\rangle \in \boldsymbol{Z} / 4 .
$$

Also from 8.1(ii),

$$
\rho_{2} \rho_{4}(l)=V^{2} \in H^{4^{*}}(B S G, \boldsymbol{Z} / 2) .
$$

Since any $\boldsymbol{Z} / 4$ manifold $f: M^{q+4 n} \rightarrow M S G_{q}$ can be made transversal by subtracting $j\left(\left[K^{q+4}, \alpha\right][C P(2 n-2)]\right)$ if necessary, $j: \Omega_{*}(, Z / 2) \rightarrow$ $\Omega_{*}(, \boldsymbol{Z} / 4)$, we see that these two properties (a), (b) uniquely characterize $\rho_{4}(l)$. But the $\boldsymbol{Z} / 4$ index class of [6] also has these properties, hence 8.1(vi) follows.

We return now to the proof of Theorem 8.4.

Proof of 8.4. (ii) We have already shown in 8.6 that, for any $Z / 2$ bordism element g: $N \rightarrow M S G$,

$$
\begin{aligned}
\varphi i([N, g]) & =i\left\langle L(N) \cdot g^{*} \Phi\left(V^{2}\right),[N]\right. \\
& =i\left\langle V^{2}(N) \cdot g^{*} \Phi\left(V^{2}\right),[N]\right\rangle \in \mathbb{Z} / 2 \subset \boldsymbol{Z} / 8 .
\end{aligned}
$$

8.4(ii) is an easy consequence of this and the multiplicativity of $V^{2}$.

Proof of 8.4. (iii) We have already shown in the proof of 8.1(iv) that $\varphi \rho_{8} \delta: \Omega_{*}(M S G, \boldsymbol{Z} / 2) \rightarrow \boldsymbol{Z}_{2}$ is the homomorphism on a $\boldsymbol{Z} / 2$ bordism which corresponds to the cohomology class $\rho_{2} \Phi(\tilde{\mathscr{L}}) \in H^{4^{*}+1}(M S G, Z / 2)$ (see Propositions A.3 and A.6 of the Appendix). In particular, $\varphi \rho_{8} \delta$ is multiplicative with respect to the index, which proves $8.4(\mathrm{iii})$.

Proof of 8.4. (i) We have four cases, depending on the dimension of $N$, namely, $\operatorname{dim}(N) \equiv 0,1,2,3(\bmod 4)$. We denote these cases 0 , 1,2 , and 3 , respectively. 
Case 0 . We are given a $Z / 8$ bordism element $f: M^{q+4 a} \rightarrow M S G_{q}$ and a closed manifold $N^{4 b}$. By definition of $\varphi$,

$$
\begin{aligned}
& \varphi([M, f][N] \\
& \quad=\varphi^{\prime \prime}([M, f][N])-i\left\langle V S q^{1} V(M \times N)\left(f^{*} \Phi(\tilde{\mathscr{K}}) \otimes 1\right),[M \times N]\right\rangle \\
& \quad=\varphi^{\prime \prime}([M, f][N])-i\left\langle V S q^{1} V(M) \cdot f^{*} \Phi(\widetilde{\mathscr{K}}),[M]\right\rangle\left\langle V^{2}(N),[N]\right\rangle .
\end{aligned}
$$

Thus it suffices to prove $\varphi^{\prime \prime}\left([M, f[N])=\varphi^{\prime \prime}([M, f]) \cdot \operatorname{index}(N) \in \boldsymbol{Z} / 8\right.$, since then

$$
\begin{aligned}
\varphi([M, f][N]) & =\left(\varphi^{\prime \prime}([M, f])-i\left\langle V S q^{1} V(M) \cdot f^{*}(\tilde{\mathscr{K}}),[M]\right\rangle\right) \cdot \operatorname{index}(N) \\
& =\varphi([M, f] \cdot \operatorname{index}[N] .
\end{aligned}
$$

Suppose $[M, f] \in \Omega_{4 *}^{\prime}(M S G, \boldsymbol{Z} / 8)=\operatorname{kernel}\left(s \delta: \Omega_{4^{*}}(M S G, \boldsymbol{Z} / 8) \rightarrow \boldsymbol{Z} / 2\right)$. Then we may assume that $f: M^{q+4 a} \rightarrow M S G_{q}$ is globally transversal, with $f^{-1}\left(B S G_{q}\right)=L^{4 a} \subset M^{q+4 a}$. By definition of $\phi^{\prime \prime}$,

$$
\varphi^{\prime \prime}([M, f])=\varphi^{\prime}([M, f])=\operatorname{index}\left(L^{4 a}\right) \in \boldsymbol{Z} / 8 .
$$

Moreover, it is obvious that $f \pi_{1}: M^{q+4 a} \times N^{4 b} \rightarrow M S G_{q}$ if globally transversal, with $\left(f \pi_{1}\right)^{-1}\left(B S G_{q}\right)=L^{4 a} \times N^{4 b}$. Necessarily,

$$
\left.s \delta\left(M^{q+4 a}, f\right]\left[N^{4 b}\right]\right)=0
$$

and, thus,

$$
\begin{aligned}
\varphi^{\prime \prime}\left(\left[M^{q+4 a}, f\right]\left[N^{4 b}\right]\right) & =\varphi^{\prime}\left(\left[M^{q+4 a}, f\right]\left[N^{4 b}\right]\right) \\
& =\operatorname{index}\left(L^{4 a} \times N^{4 b}\right) \\
& =\operatorname{index}\left(L^{4 a}\right) \cdot \operatorname{index}\left(N^{4 b}\right) \\
& =\varphi^{\prime \prime}[M, f] \cdot \operatorname{index}(N),
\end{aligned}
$$

as desired.

Finally, by linearity of $\rho$, it suffices to check the case $[M, f]=$ $i\left(\left[K^{q+4}, \alpha\right][C P(2 a-2)]\right)$. But we have already shown in 8.6 that

$$
\begin{aligned}
& \varphi i\left(\left[K^{q+4}, \alpha\right][C P(2 a-2)]\right) \\
& =i\left\langle L\left(K^{q+4} \times C P(2 a-2)\right) \cdot\left(\alpha^{*} \Phi\left(V^{2}\right) \otimes 1\right),\right. \\
& \left.\quad\left[K^{q+4} \times C P(2 a-2)\right]\right\rangle=0
\end{aligned}
$$

by 8.3 (ii) and the fact $L\left(K^{q+4}\right)=1$ (this is also obvious from the definition of $\varphi$ ), and we have shown that

$$
\begin{aligned}
& \varphi i\left(\left[K^{q+4}, \alpha\right][C P, \alpha][C P(2 a-2)]\left[N^{4 b}\right]\right) \\
& =i\left\langle\left(K^{q+4} \times C P(2 a-2) \times N^{4 b}\right)\right. \\
& \left.\quad \cdot\left(\alpha^{*} \Phi\left(V^{2}\right) \otimes 1 \otimes 1\right),\left[K^{q+4} \times C P(2 a-2) \times N^{4 b}\right]\right\rangle \\
& \quad=0
\end{aligned}
$$


by $8.3(\mathrm{ii})$ and the fact $L\left(K^{q+4}\right)=1$. This proves Case 0 of 8.4(i).

In all three cases below, it is easy to check that $s \delta([M, f][N])=0$. Thus,

$$
\begin{aligned}
& \varphi([M, f][N]) \\
& \quad=\varphi^{\prime}([M, f][N])-i\left\langle V S q^{1} V(M \times N)\left(f^{*} \tilde{\mathscr{K}} \otimes 1\right),[M \times N]\right\rangle .
\end{aligned}
$$

Also, in Cases 1,2 , and 3 , index $(N)=0$, so what we must show is that $\varphi([M, f][N])=0$.

Case 2. We have $f: M^{q+4 a-2} \rightarrow M S G_{q}$ and $N^{4 b+2}$, where $M$ is a $\boldsymbol{Z} / 8$ manifold and $N$ is closed. For dimensional reason,

$$
\left\langle V S q^{1} V(M \times N)\left(f^{*} \tilde{\mathscr{L}} \otimes 1\right),[M \times N]\right\rangle=0 .
$$

On the other hand, by Theorem 3.4 we may assume that $f: M \rightarrow M S G_{q}$ is globally transversal, with $f^{-1}\left(B S G_{q}\right)=L^{4 a-2} \subset M^{q+4 a-2}$. Then $f \pi_{2}$ : $M \times N \rightarrow M S G_{q}$ is globally transversal, with $\left(f \pi_{2}\right)^{-1}\left(B S G_{q}\right)=L^{4 a-2} \times$ $N^{4 b+2}$. Thus $\varphi^{\prime}([M, f][N])=\operatorname{index}\left(L^{4 a-2} \times N^{4 b+2}\right)=0$, which proves Case 2.

Case 1. We have $f: M^{q+4 a-1} \rightarrow M S G_{q}$ and $N^{4 b+1}$. We know from the structure of $\Omega_{*}(p t)$ that $8[N]=0 \in \Omega_{4 b+1}(p t)$. Thus there is a $Z / 8$ manifold $\widetilde{N}$ with $\delta \widetilde{N}=N$. We will now use the fact that the composition $\rho_{8} \delta$ behaves like a derivation. Thus

$$
\rho_{8} \delta([M, f] \cdot[\tilde{N}])=[M, f] \cdot[N]+\left[\delta M,\left.f\right|_{\delta M}\right] \cdot[\tilde{N}] \in \Omega_{q+4(a+b)}\left(M S G_{q}, \boldsymbol{Z} / 8\right) .
$$

The proof of Case 2 above show that $\varphi\left(\left[\delta M,\left.f\right|_{\delta M}\right][\tilde{N}]\right)=0$. Thus, by the proof of 8.1(iv),

$$
\begin{aligned}
& \varphi([M, f][N] \\
& \quad=\varnothing \rho_{8} \delta([M, f][\tilde{N}]) \\
& \quad=s_{8}([M, f] \cdot[\tilde{N}])-i\left\langle V S q^{1} V(\delta(M \times \tilde{N})) \cdot\left(f^{*} \tilde{\mathscr{K}} \otimes 1\right),[\delta(M \times \tilde{N})]\right\rangle \\
& \quad=i\left(\left(s_{8}[M, f] \cdot d(\delta \tilde{N})\right)-\left\langle V^{2}(M) \cdot f^{*} \tilde{\mathscr{K}},[M]\right\rangle\left\langle V S q^{1} V(\delta \tilde{N}),[\delta \tilde{N}]\right\rangle\right) \\
& \quad=0 .
\end{aligned}
$$

This proves Case 1.

Case 3. We have $f: M^{q+4 a+1} \rightarrow M S G_{q}$ and $N^{4 b-1}$. As in Case 1, we can find a $Z / 8$ manifold $\tilde{N}$, with $\delta \tilde{N}=N$. Then $\rho_{8} \delta([M, f][\tilde{N}])=$ $[M, f][N]+\left[\delta M,\left.f\right|_{\delta M}\right] \cdot[\tilde{N}]$, hence

$$
\begin{aligned}
\varphi([M, f][N]) & =\varphi \rho_{8} \delta([M, f][\tilde{N}])-\varphi\left(\left[\delta M,\left.f\right|_{\delta M}\right][\tilde{N}]\right) \\
& =\varphi \rho_{8} \delta[M, f] \cdot \operatorname{index}(\tilde{N})-\varphi \rho_{8}\left[\partial M,\left.f\right|_{\delta M}\right] \cdot \operatorname{index}(\tilde{N}) \\
& =0 .
\end{aligned}
$$


The second equality uses both the proof of 8.4(iii), which implies that $\varphi \rho_{8} \delta$ is multiplicative with respect to the index, and the proofs of 8.4(ii) and Case 0 of 8.4(i) above, which imply that $\varphi\left(\left[\widetilde{M}^{q+4 a}, \widetilde{f}\right]\left[\widetilde{N}^{4 b}\right]\right)=$ $\varphi\left[\widetilde{M}^{q+4 a}, \widetilde{f}\right] \cdot \operatorname{index}\left(\widetilde{N}^{4 b}\right)$, for any $Z / 8$ manifolds $\widetilde{M}^{q+4 a}$ and $\widetilde{N}^{4 b}$. This proves Case 3, and completes the proof of Theorem 8.4.

REMARK 8.8. The definition of $\varphi: \Omega_{4^{*}}(M S G, Z / 8) \rightarrow Z / 8$ can be extended directly to $P L$-bordism, $\varphi: \Omega_{4}^{P L}(M S G, Z / 8) \rightarrow Z / 8$. Moreover, the formula of Theorem 8.5 is true for $M$ any $P L Z / 8$ manifold. This assertion does not follow from the product formulae 8.4, however. One needs the more delicate formulae

$$
\varphi(i([\tilde{M}, \tilde{f}][\tilde{N}]))=\varphi(i[\tilde{M}, \tilde{f}]) \cdot \operatorname{index}(\tilde{N}) \in \boldsymbol{Z} / 2^{r} \subset \boldsymbol{Z} / 8
$$

and

$$
\varphi\left(\rho_{8} \partial([\widetilde{P}, \widetilde{F}][\widetilde{Q}])\right)=\varphi\left(\rho_{8} \delta[\widetilde{P}, \widetilde{F}]\right) \cdot \operatorname{index}(\widetilde{Q}) \in \boldsymbol{Z} / 2^{r} \subset \boldsymbol{Z} / 8,
$$

where $\tilde{M}, \tilde{N}, \widetilde{P}, \widetilde{Q}$ are $Z / 2^{r}$ manifolds, $r \leqq 3, \operatorname{dim}(\widetilde{M} \times \tilde{N}) \equiv 0(\bmod 4)$ and $\operatorname{dim}(\widetilde{P} \times \widetilde{Q}) \equiv 1(\bmod 4)$. The reason we need stronger formulae to deduce 8.5 for $P L$-manifolds is that $\Omega_{*}^{P L}(p t, Z / 8)$ is not generated by oriented manifolds and $\boldsymbol{Z} / 2$ manifolds. (See the Appendix, Remark A.14.) In the next section, we will establish generalizations of these more difficult product formulae, which will thus prove 8.5 for $P L$ manifolds.

9. The Whitney sum formula for the class $l$. In this section, we will establish Property 8.1(iii) of the class $l \in H^{4 *}(B S G, Z / 8)$. That is, if $\xi$ and $\eta$ are two oriented spherical fibrations, we prove

$$
\begin{aligned}
l(\xi \times \eta)= & l(\xi) \otimes l(\eta)+i\left(V S q^{1} V(\xi) \otimes \tilde{\mathscr{K}}(\eta)\right. \\
& \left.+\tilde{\mathscr{K}}(\xi) \otimes V S q^{1} V(\eta)\right) \in H^{4^{*}}(B \xi \times B \eta, Z / 8),
\end{aligned}
$$

where

$$
i: H^{*}(, Z / 2) \longrightarrow H^{*}(, Z / 8) \text {. }
$$

To establish 9.1, we need the Whitney sum formula for the class $\tilde{\mathscr{\kappa}} \in H^{4^{*}-1}(B S G, Z / 2)$.

$$
\begin{aligned}
\mathscr{K}(\xi \times \eta)= & \tilde{\mathscr{K}}(\xi) \otimes V^{2}(\eta) \\
& +V^{2}(\xi) \otimes \tilde{\mathscr{K}}(\eta) \in H^{4^{*-1}}(B \xi \times B \eta, Z / 2) .
\end{aligned}
$$

9.2 will be proved in $\S 10$ by giving a homotopy theoretic definition of the class $\mathscr{K}$ in terms of functional cohomology operations, and then proving a product formula for the relevant functional operations. A proof of 9.2 could also be given by reducing 9.2 to 
known formula for the Kervaire surgery obstruction.

As a first step in the proof of 9.1, we describe generators of $H_{*}\left(X \times Y, Z / 2^{r}\right)$.

Lemma 9.3. $H_{*}\left(X \times Y, Z / 2^{r}\right)$ is generated by the Hurewicz image of

(i ) "products" $j([M, f][N, g])$, where $M$ and $N$ are $Z / 2^{s}$-manifolds, $s \leqq r, f: M \rightarrow X, g: N \rightarrow Y$, and $j: \Omega_{*}\left(, Z / 2^{s}\right) \rightarrow \Omega_{*}\left(, Z / 2^{r}\right)$, and

(ii) Bocksteins of products $\rho_{2^{r}} \delta([P, f][Q, g])$, whese $P$ and $Q$ are $Z / 2^{s}$-manifolds, $s<r, f: P \rightarrow X$, and $g: Q \rightarrow Y$.

Note in (ii) that, if

$$
s \geqq r, \rho_{2^{r}} \delta([P, f][Q, g])=\rho_{2^{r}} \delta[P, f] \cdot \rho_{2^{r}}[Q, g]+\rho_{2^{r}}[P, f] \cdot \rho_{2^{r}} \delta[Q, g] .
$$

A simple proof of Lemma 9.3 can be given by studying tensor products of elementary chain complexes

$$
\left(0 \longrightarrow Z \stackrel{2^{a}}{\longrightarrow} Z \longrightarrow 0\right) \otimes\left(0 \rightarrow Z \stackrel{2^{b}}{\longrightarrow} Z \longrightarrow 0\right),
$$

since by a classical "standard basis theorem" such complexes account for the 2-torsion in $H_{*}(X, Z)$ and $H_{*}(Y, Z)$. We leave the details to the reader.

To prove 9.1 , it clearly suffices to prove

$$
\Phi l(\xi \times \eta)
$$

$$
\begin{aligned}
= & \Phi\left(l(\xi) \otimes l(\eta)+i\left(V S q^{1} V(\xi) \otimes \tilde{\mathscr{K}}(\eta)+\tilde{\mathscr{K}}(\xi) \otimes V S q^{1} V(\eta)\right)\right) \\
& \in H^{4^{*}}(T \xi \wedge T \eta, Z / 8)=\operatorname{Hom}\left(H_{4^{*}}(T \xi \wedge T \eta, Z / 8), Z / 8\right) .
\end{aligned}
$$

By Lemma 9.3, it thus suffices to evaluate both sides of 9.4 on "products" $j([M, f][N, g]), M, N Z / 2^{s}$-manifolds, $s \leqq 3, f: M \rightarrow T \xi$, $g: N \rightarrow T \eta$, and Bocksteins of products $\rho_{8} \delta([P, f][Q, g]), P, Q Z / 2^{s}$-manifolds, $s<3, f: P \rightarrow T \xi, g: Q \rightarrow T \eta$.

In $\S 8$, we defined a homomorphism $\phi_{\xi \times \eta}: \widetilde{\Omega}_{4^{*}}(T \xi \wedge T \eta, Z / 8) \rightarrow Z / 8$, and the graded class $\Phi(l(\xi \times \eta))=\Phi\left(\sum_{n \geqq 0} l_{n}(\xi \times \eta)\right)$ was defined so that

$$
\phi_{\xi \times \eta}([X, h])=\left\langle L(X) \cdot h^{*} \Phi(l(\xi \times \eta)),[X]\right\rangle \in Z / 8
$$

for all $Z / 8$-manifolds $h: X \rightarrow T \xi \wedge T \eta$. By induction on $n$, where $l=\sum_{n \geqq 0} l_{n}, 9.4$ follows if we show

$$
\begin{aligned}
\phi_{\xi \times \eta}([X, h])= & \left\langle L(X) \cdot h^{*} \Phi(l(\xi) \otimes l(\eta)),[X]\right\rangle \\
& +4\left\langleV ^ { 2 } ( X ) h ^ { * } \Phi \left( V S q^{1} V(\xi) \otimes \tilde{\mathscr{C}}(\eta)\right.\right. \\
& \left.\left.+\widetilde{\mathscr{K}}(\xi) \otimes V S q^{1} V(\eta)\right), \rho_{2}[X]\right\rangle
\end{aligned}
$$

for $[X, h]=j([M, f][N, g])$ and for $\left.[X, h]=\rho_{8} \delta(P, f][Q, g]\right), M, N, P, Q$ 
as above, $4: Z / 2 \rightarrow Z / 8$.

We first dispose of cases $[X, h]=j([M, f][N, g])$, where $M$ and $N$ are $Z / 2$ - or $Z / 4$-manifolds. The second term on the RHS of (9.5) vanishes. Thus we must show

$$
\begin{aligned}
& \left\langle L(M \times N)(f \times g)^{*} \Phi\left(\rho_{2^{s}} l(\xi \times \eta)\right),[M \times N]\right\rangle \\
& \left.\quad=\left\langle L(M \times N)(f \times g)^{*} \Phi\left(\rho_{2^{s}} l(\eta)\right) \otimes \rho_{2^{s}} l(\eta)\right),[M \times N]\right\rangle \in Z / 2^{s}, s=1,2 .
\end{aligned}
$$

But we know $\rho_{2} l(\xi \times \eta)=\rho_{2} l(\xi) \otimes \rho_{2} l(\eta)$, since, by 8.1(ii), $\rho_{2}(l)=V^{2}$. Also, we know that $\rho_{4} l(\xi \times \eta)=\rho_{4} l(\xi) \otimes \rho_{4} l(\eta)$, since, by 8.1(vi), $\rho_{4}(l)$ is the $Z / 4$-index class defined in [6].

We will now specialize to $T \xi=T \eta=M S G$. By naturality of the class $l$, this is sufficient. We write $f: M^{a} \rightarrow M S G$ instead of $f: M^{q+a} \rightarrow$ $M S G_{q}$. Of course, we work in the stable range, $a<q-2$. There should be no confusion if we write $L^{a}=f^{-1}(B S G) \subset M^{a}$, when $f: M^{a} \rightarrow$ $M S G$ is globally transversal.

We are left with five cases in 9.5:

Case 1. $[X, h]=\left[M^{4 a+2}, f\right]\left[N^{4 b+2}, g\right], M, N Z / 8$-manifolds.

Case 2. $[X, h]=\left[M^{4 a}, f\right]\left[N^{4 b}, g\right], M, N Z / 8$-manifolds.

Case 3. $[X, h]=\left[M^{4 a+1}, f\right]\left[N^{4 b-1}, g\right], M, N Z / 8$-manifolds.

Case 4. $\left.[X, h]=\rho_{8} \delta\left(P^{4 a+2}, f\right]\left[Q^{4 b-1}, g\right]\right), P, Q Z / 2$ - or $Z / 4$-manifolds.

Case 5. $[X, h]=\rho_{8} \delta\left(\left[P^{4 a+1}, f\right]\left[Q^{4 b}, g\right]\right), P, Q Z / 2$ - or $Z / 4$-manifolds.

We will concentrate on evaluating the RHS of $9.5, \phi([X, h])$, in these five cases, and leave to the reader the algebraic computation of the RHS of 9.5 .

Proof of Case 1. Recall from $\S 8$ the definition of

$$
\dot{\phi}: \widetilde{\Omega}_{4^{*}}(M S G, Z / 8) \longrightarrow Z / 8 \text {. }
$$

If $f: X^{4 n} \rightarrow M S G$ is globally transversal, $X$ a $Z / 8$-manifold, with $Y^{4 n}=$ $h^{-1}(B S G) \subset X$, then

$$
\dot{\phi}([X, h])=\operatorname{index}\left(Y^{4 n}\right)-4\left\langle V S q^{1} V(X) \cdot h^{*} \Phi(\widetilde{K}),[X]\right\rangle \in Z / 8 .
$$

In Case $1,[X, h]=\left[M^{4 a+2}, f\right]\left[N^{4 b+2}, g\right]$. We may assume that both $f: M^{4 a+2} \rightarrow M S G$ and $g: N^{4 b+2} \rightarrow M S G$ are globally transversal, since there is no obstruction in this dimension. Let $K^{4 a+2}=f^{-1}(B S G) \subset M$, $L^{4 b+2}=g^{-1}(B S G) \subset N$. Then $f \times g: M \times N \rightarrow M S G \wedge M S G$ is globally 
transversal, and $(f \times g)^{-1}(B S G \times B S G)=K \times L$. Moreover, by 9.6 and 9.2 , and since

$$
\begin{aligned}
V S q^{1} V(M \times N)= & V^{2}(M) \otimes V S q^{1}(N)+V S q^{1} V(M) \otimes V^{2}(N), \\
& \left.\times \phi\left(M^{4 a+2}, f\right]\left[N^{4 b+2}, g\right]\right) \\
= & \operatorname{index}(K \times L)-4\left\langle V S q^{1} V(M \times N) \cdot(f \times g)^{*}\right. \\
& \left.\times\left(\Phi\left(\widetilde{\mathscr{K}} \otimes V^{2}+V^{2} \otimes \widetilde{\mathscr{K}}\right)\right),[M \times N]\right\rangle \\
= & \text { index }(K \times L)=0 .
\end{aligned}
$$

The reader can check by dimensional considerations that the RHS of 9.5 also vanishes in Case 1.

Proof of Case 2. We first argue that we may assume both $f: M^{4 a} \rightarrow M S G$ and $g: N^{4 b} \rightarrow M S G$ are globally transversal. Let $\alpha$ : $K^{4} \rightarrow M S G$ be the nontransversal $Z / 2$-manifold used in 8.3. ([K $\left.K^{4} \alpha\right]$ will be precisely constructed in $\S 10$; this is the step where we use $T \xi=T \eta=M S G$.) In any dimension $4 a$, we get a nontransversal manifold $\alpha \pi_{1}: K^{4} \times C P(2 a-2) \rightarrow M S G$. If $f: M^{4 a} \rightarrow M S G$ is not transversal, write

$$
\left[M^{4 a}, f\right]\left[N^{4 b}, g\right]=\left[M^{\prime}, f^{\prime}\right][N, g]+j\left(\left(\left[K^{4}, \alpha\right][C P(2 a-2)]\left(\rho^{2}[N, g]\right)\right),\right.
$$

where $\left[M^{\prime}, f^{\prime}\right]=[M, f]-j\left(\left[K^{4}, \alpha\right][C P(2 \alpha-2)]\right)$. Since both sides of formula 9.5 are additive in $[X, h]$, this reduces the general Case 2 to the case $[X, h]=\left[M^{\prime}, f^{\prime}\right][N, g]$, where $f^{\prime}: M^{\prime} \rightarrow M S G$ is transversal. (We have already dealt with the case $[X, h]=j(Z / 2$-manifold).) Similarly, we may assume that $g: N^{4 b} \rightarrow M S G$ is transversal.

Now, assuming that $f: M^{4 a} \rightarrow M S G$ and $g: N^{4 b} \rightarrow M S G$ are transversal, let $K^{4 a}=f^{-1}(B S G) \subset M, L^{4 b}=g^{-1}(B S G) \subset N$. Then, by 9.6 and 9.2 ,

$$
\begin{aligned}
\phi( & {[M, f][N, g]) } \\
= & \operatorname{index}(K \times L)-4\left\langle V S q^{1} V(M \times N)(f \times g)^{*}\right. \\
& \left.\times \Phi\left(\tilde{\mathscr{K}} \otimes V^{2}+V^{2} \otimes \widetilde{\mathscr{K}}\right),[M \times N]\right\rangle \\
= & \operatorname{index}(K \times L)-4\left\langle V^{2}(M) \cdot f^{*} \Phi\left(V^{2}\right),[M]\right\rangle\left\langle V S q^{1} V(N) \cdot g^{*} \Phi(\tilde{\mathscr{K}}),[N]\right\rangle \\
& -4\left\langle V S q^{1} V(M) \cdot f^{*} \Phi(\tilde{\mathscr{K}}),[M]\right\rangle\left\langle V^{2}(N) \cdot g^{*} \Phi\left(V^{2}\right),[N]\right\rangle \\
= & \left(\operatorname{index}(K)-4\left\langle V S q^{1} V(M) \cdot f^{*} \Phi(\tilde{\mathscr{K}}),[M]\right\rangle\right) \\
& \times\left(\operatorname{index}(L)-4\left\langle V S q^{1} V(N) \cdot g^{*} \Phi(\tilde{\mathscr{K}}),[N]\right\rangle\right) \\
= & \phi\left(\left[M^{4 a}, f\right] \cdot \phi\left(N^{4 b}, g\right]\right) \in Z / 8 .
\end{aligned}
$$

(We have used the identities $\left\langle V^{2}(M) \cdot f^{*} \Phi\left(V^{2}\right),[M]\right\rangle=\left\langle V^{2}(K),\left[K^{4 a}\right]\right\rangle=$ index $\left(K^{4 a}\right)(\bmod 2)$, and similarly, $\left\langle V^{2}(N) \cdot g^{*} \Phi\left(V^{2}\right),[N]\right\rangle=\operatorname{index}\left(L^{4 b}\right)$ $(\bmod 2)$. 
It is easy to see that this agrees with the RHS of (9.5).

Case 3 is very difficult, without making use of the results of Cases 4 and 5, so we postpone it until the end of the section.

To study Cases 4 and 5, we recall that, if $\widetilde{h}: \widetilde{X}^{4 n+1} \rightarrow M S G$ is a $Z / 2^{s}$-manifold, then we may always assume $\left.\tilde{h}\right|_{\delta \tilde{X}}: \delta \tilde{X} \rightarrow M S G$ is globally transversal, with $Y^{4 n}=\left(\left.\widetilde{h}\right|_{\delta \widetilde{X}}\right)^{-1}(B S G) \subset \delta \widetilde{X}$. If $[\widetilde{X}, \tilde{h}]=[P, f][Q, g]$, then $\rho_{2} \delta[\tilde{X}, \tilde{h}]=\delta[P, f] \cdot \rho_{2}[Q, g]+\rho_{2}[P, f] \cdot \delta[Q, g]$. The formula 9.6 for $\phi \rho_{8} \delta[P, f][Q, g]$ then becomes (by 9.2)

$$
\phi \rho_{8} \delta([P, f][Q, g])=\operatorname{index}(Y)
$$

$$
\begin{aligned}
& -4\left\langle V S q^{1} V(\delta P \times Q)(f \times g)^{*} \Phi\left(V^{2} \otimes \tilde{\mathscr{K}}+\tilde{\mathscr{K}} \otimes V^{2}\right),[\delta P \times Q]\right\rangle \\
& -4\left\langle V S q^{1} V(P \times \delta Q)(f \times g)^{*} \Phi\left(V^{2} \otimes \tilde{\mathscr{K}}+\tilde{\mathscr{K}} \otimes V^{2}\right),[P \times \delta Q]\right\rangle .
\end{aligned}
$$

Moreover, we proved in Lemma 3.7 that

$$
2^{3-s} s_{2^{s}}([P, f][Q, g])=\operatorname{index}(Y) \in Z / 8,
$$

where $s_{2^{s}}: \widetilde{\Omega}_{4^{*}+1}\left(M S G, Z / 2^{s}\right) \rightarrow Z / 2^{s}$ is the transversality obstruction of $\S 3$.

Proof of Case 4. We may assume that $f: P^{4 a+2} \rightarrow M S G$ is globally transversal, with $K^{4 a+2}=f^{1}(B S G) \subset P, K^{4 a+2}$ a $Z / 2^{s}$-PD space, $s=1$ or 2. We consider two subcases for products $\left[P^{4 a+2}, f\right]\left[Q^{4 b-1}, g\right]$.

Subcase 1. $g: Q^{4 b-1} \rightarrow M S G$ transversal, $L^{4 b-1}=f^{-1}(B S G) \subset Q, L$ and $Q Z / 2^{s}$-manifolds.

Subcase 2. $Q^{4 b-1}$ a closed manifold, $g: Q^{4 b-1} \rightarrow M S G$.

Since there is a nontransversal example on a closed manifold, $\alpha: S^{3} \rightarrow M S G$ (e.g., $\left[S^{3}, \alpha\right]=\delta\left[K^{4}, \alpha\right]$, where $\left[K^{4}, \alpha\right]$ is as in 8.3), we see by linearity that Subcases 1 and 2 suffice.

Subcase 1. In this case, $f \times g: P^{4 a+2} \times Q^{4 b-1} \rightarrow M S G \wedge M S G$ is transversal, with $(f \times g)^{-1}(B S G \times B S G)=K^{4 a+2} \times L^{4 b-1}$. Since $2^{s} \delta\left(K^{4 a+2} \times L^{4 b-1}\right)$ is a boundary, index $\left(\delta\left(K^{4 a+2} \times L^{4 b-1}\right)\right)=0$. The hypothesis $g: Q^{4 b-1} \rightarrow M S G$ transversal also implies $\left\langle V^{2}(Q) \cdot g^{*} \Phi(\widetilde{K})\right.$, $[Q]\rangle=0$. We thus conclude from 9.7 that $\phi \rho_{8} \delta\left(\left[P^{4 a+2}, f\right]\left[Q^{4 b-1}, g\right]\right)=0$.

To evaluate the first term on the RHS of 9.5 , it is helpful to use the identity

$$
\begin{aligned}
& \left\langle L(\delta \widetilde{X}) \cdot\left(\left.h\right|_{\delta \widetilde{X}}\right)^{*} \Phi(l(\xi) \otimes l(\eta)),[\delta \widetilde{X}]\right\rangle \\
& \quad=2^{3-s}\left\langle L(\widetilde{X}) \cdot h^{*} \Phi\left(\rho_{2^{s}} \beta l(\xi) \otimes \rho_{2^{s}} l(\eta)+\rho_{2^{s}} l(\xi) \otimes \rho_{2^{s}} \beta l(\eta)\right),[\tilde{X}]\right\rangle,
\end{aligned}
$$

where $\tilde{h}: \tilde{X} \rightarrow T \xi \wedge T \eta$ is a $Z / 2^{s}$-manifold, $s \leqq 3$, and $\beta: H^{*}(, Z / 8) \rightarrow$ 
$H^{*+1}(, Z)$ is the Bockstein. Thus the first term on the RHS of 9.5 vanishes if $[\tilde{X}, \tilde{h}]=\left[P^{4 a+2}, f\right]\left[Q^{4 b-1}, g\right]$. The second term is easily calculated, using $\rho_{2} \delta[P \times Q]=[\delta P] \cdot \rho_{2}[Q]+\rho_{2}[P] \cdot[\delta Q]$, and also vanishes in Subcase 1, as desired.

Subcase 2. If $Q$ is closed, $\delta\left(P^{4 a+2} \times Q^{4 b-1}\right)=\delta P \times Q$. If $g: Q^{4 b-1} \rightarrow$ $M S G$ is not transversal, then the map $f \times g: \delta P \times Q \rightarrow M S G \wedge M S G$ is not transversal, although it is bordant to a globally transversal map. We want to compute index $(Y) \in Z / 8$, where $Y=h^{-1}(B S G \times B S G)$, $h: X^{4 a+4 b} \rightarrow M S G \wedge M S G$ a transversal map bordant to $f \times g: \delta P \times$ $Q \rightarrow M S G \wedge M S G$.

Let $Q^{4 b-1}=U_{1} \bigcup_{\partial} U_{2}$ be a partition of $g: Q^{4 b-1} \rightarrow M S G$ (see $\S 3$ ). That is, $U_{1}$ and $U_{2}$ are codimension 0 -submanifolds of $Q,\left.g\right|_{U_{1}}: U_{1} \rightarrow$ $M S G$ is globally transversal with respect to the mapping cylinder $M_{\gamma} \subset M S G$ of the universal bundle $\gamma \rightarrow B S G$, and $\left.g\right|_{U_{2}}: U_{2} \rightarrow M S G$ is globally transversal with respect to a smaller mapping cylinder $M_{\gamma}, \subset M_{r}$.

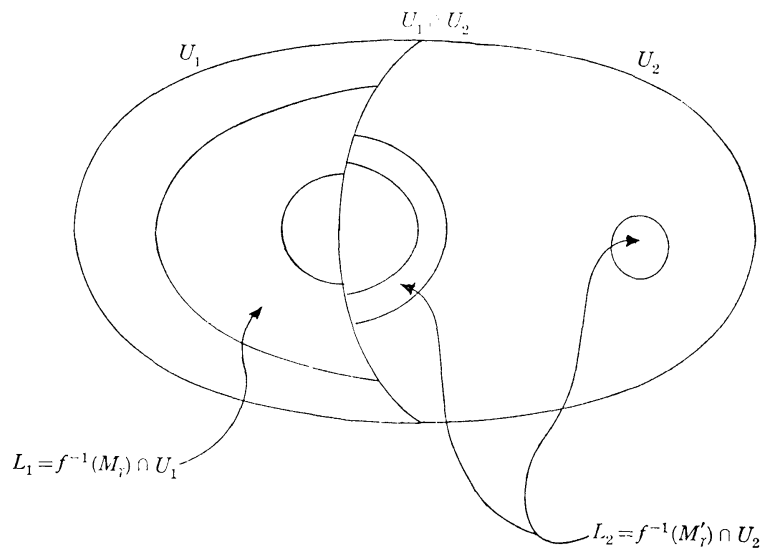

Let $L_{1}=\left(\left.g\right|_{U_{1}}\right)^{-1}\left(M_{\gamma}\right), L_{2}=\left(\left.g\right|_{U_{2}}\right)^{-1}\left(M_{\gamma^{\prime}}\right)$. Then by Lemma 1.4, the inclusion $\partial L_{2} \rightarrow \partial L_{1}$ is a degree one normal map of closed, $4 b-2$-dimensional PD spaces. Moreover, the global transversality obstruction $s\left(Q^{4 b-1}, g\right) \in Z / 2$ is equal to the Kervaire obstruction $S\left(\partial L_{2} \rightarrow \partial L_{1}\right) \in Z / 2$.

Since $\left.f\right|_{\delta P}: \delta P \rightarrow M S G$ is globally transversal, the partition of $[Q, g]$ induces a partition of $[\delta P \times Q, f \times g]$. Namely,

$$
\delta P \times Q=\delta P \times U_{1} \bigcup_{\partial} \delta P \times U_{2} .
$$

In the intersection, $\delta P \times\left(U_{1} \cap U_{2}\right)$, we get a normal map of closed, $4 a+4 b-1$-dimensional PD spaces $\delta K \times \partial L_{2} \rightarrow \delta K \times \partial L_{1}$. (Recall $K=$ $f^{-1}(B S G) \subset P^{4 a+2}$.) This normal map has associated an invariant $\theta\left(\delta K \times \partial L_{2} \rightarrow \delta K \times \partial L_{1}\right) \in Z / 8$, and, by Lemma 3.11, the index we 
need to compute is given by

$$
\begin{aligned}
\operatorname{index}(Y)= & \operatorname{index}\left(\delta K \times L_{1}\right)+\operatorname{index}\left(\delta K \times L_{2}\right) \\
& -\theta\left(\delta K \times \partial L_{2} \rightarrow \delta K \times \partial L_{1}\right) \in Z / 8 .
\end{aligned}
$$

Of course, index $\left(\delta K \times L_{1}\right)=$ index $\left(\delta K \times L_{2}\right)=0$. By Lemma 3.12,

$$
\theta\left(\delta K \times \partial L_{2} \longrightarrow \delta K \times \partial L_{1}\right)=4 d(\delta K) \cdot S\left(\partial L_{2} \rightarrow \partial L_{1}\right) .
$$

Since

$$
\begin{aligned}
d(\delta K) & =\left\langle V S q^{1} V(\delta K),[\delta K]\right\rangle \\
& =\left\langle V^{2}(\delta P) f^{*} \Phi\left(V S q^{1} V\right)+V S q^{1} V(\delta P) \cdot f^{*} \Phi\left(V^{2}\right),[\delta P]\right\rangle \in Z / 2,
\end{aligned}
$$

and, since $S\left(\partial L_{2} \rightarrow \partial L_{1}\right)=s\left[Q^{4 b-1}, g\right]=\left\langle V^{2}(Q) \cdot g^{*} \Phi(\tilde{\mathscr{K}}),[Q]\right\rangle \in Z / 2$, we obtain from 9.7

$$
\phi\left(\left[P^{4 a+2}, f\right]\left[Q^{4 b-1}, g\right]\right)=4\left\langle V^{2}(\delta P) \cdot f^{*} \Phi\left(V^{2}\right),[\delta P]\right\rangle\left\langle V^{2}(Q) \cdot{ }^{*} \Phi(\tilde{\mathscr{K}}),[Q]\right\rangle .
$$

It is easy to check that this agrees with the RHS of 9.5.

Proof of Case 5. We deal separately with the following subcases.

Subcase 1. $P, Q Z / 2^{s}$-manifolds, $s \leqq 3$, and $g: Q^{4 b} \rightarrow M S G$ globally transversal.

Subcase 2. $P, Q Z / 2$-manifolds, $f: P^{4 a+1} \rightarrow M S G$ transversal.

Subcase 3. $P, Q Z / 2$-manifolds, $[P, f]=\left[L^{6}\right]\left[S^{3}, \alpha\right]$, where $\left[S^{3}, \alpha\right]=$ $\delta\left[K^{4}, \alpha\right],[K, \alpha]$ as in 8.3 , and $L^{6}$ is a $Z / 2$ 6-manifold with $d\left(\delta L^{6}\right)=$ $1 \in Z / 2$. Thus $s_{2}[P, f]=d\left(\delta L^{6}\right) \cdot s\left[S^{3}, \alpha\right]=1 \in Z / 2$.

The point is, Subcases 2 and 3 imply Case 5 for all $Z / 2$-manifolds $P$ and $Q$, by linearity. The fact that the nontransversal example $\left[L^{6}\right]\left[S^{3}, \alpha\right]$ has dimension 9 rather than 5 is no drawback since both sides of (9.5) "commute with crossing with $C P(2) . "$ Subcase 1 is then sufficient for all $Z / 4$ - and $Z / 8$-manifolds since there is the nontransversal example $[Q, g]=j\left[K^{4}, \alpha\right], j: \Omega_{*}(, Z / 2) \rightarrow \Omega_{*}\left(, Z / 2^{s}\right), s=2$ or 3 , which reduces the nontransversal $Z / 2^{s}$-case to the $Z / 2$-case.

Subcase 1. Consider $f: P^{4 a+1} \rightarrow M S G$. We may assume that there is a partition $P^{4 a+1}=U_{1} \cup U_{2}$ such that $\partial P \subset U_{1},\left.f\right|_{U_{1}}: U_{1} \rightarrow M S G$ is globally transversal with respect to the mapping cylinder $M_{\gamma}$, and $\left.f\right|_{U_{2}}: U_{2} \rightarrow M S G$ is globally transversal with respect to $M_{r}^{\prime} \subset M_{r}$. 


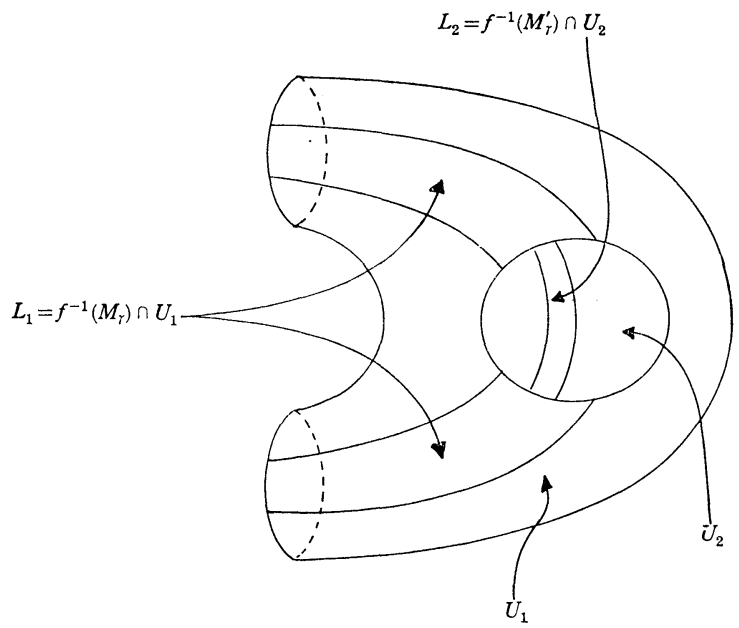

(We may assume that $\partial P \subset U_{1}$, roughly because there is no obstruction to transversality on $\partial P$.) Let $L_{1}=f^{-1}\left(M_{\gamma}\right) \cap U_{1}, L_{2}=f^{-1}\left(M_{\gamma}^{\prime}\right) \cap U_{2}$. We will use the notation $\delta L_{1}=L_{1} \cap \delta P_{1}$ and $\partial L_{1}=L_{1} \cap U_{2}$. Thus the inclusion $\partial L_{2} \rightarrow \partial L_{1}$ is a degree one normal map of closed, $4 a$-dimensional $\mathrm{PD}$ spaces.

We are assuming that $g: Q^{4 b} \rightarrow M S G$ is transversal. Let $K^{4 b}=$ $g^{-1}(B S G) \subset Q$. Now, $\delta(P \times Q)=\delta P \times Q \bigcup_{2^{s_{\delta} P \times \delta} Q} P \times \delta Q$. The partition $P=U_{1} \cup U_{2}$ induces a partition

$$
\delta(P \times Q)=\left(\delta P \times Q{\underset{2}{s_{\delta} P \times \delta Q}}_{\bigcup_{1}} U_{1} \times \delta Q\right) \cup U_{2} \times \delta Q=V_{1} \cup V_{2} .
$$

In the intersection, $V_{1} \cap V_{2}=\left(U_{1} \cap U_{2}\right) \times \delta Q$, we have the degree one normal map $\partial L_{2} \times \delta K \rightarrow \partial L_{1} \times \delta K$. On side $V_{1}$ of the partition, we have $\partial L_{1} \times \delta K=\partial\left(\delta L_{1} \times K \bigcup_{2^{s} \delta L_{1} \times \delta K} L_{1} \times \delta K\right)$. On side $V_{2}$ of the partition, we have $\partial L_{2} \times \delta K=\partial\left(L_{2} \times \delta K\right)$.

We know that $f \times\left. g\right|_{\delta}: \delta(P \times Q) \rightarrow M S G \wedge M S G$ is bordant to a globally transversal map $h: X \rightarrow M S G \wedge M S G$. Let

$$
Y=h^{-1}(B S G \times B S G) .
$$

From Lemmas $3.11,3.12$, we know that

$$
\begin{aligned}
\operatorname{index}(Y)= & \operatorname{index}\left(\delta L_{1} \times K \underset{2^{s_{\delta} L_{1} \times \delta K}}{\longrightarrow} L_{1} \times \delta K\right)+\operatorname{index}\left(L_{2} \times \delta K\right) \\
& -\theta\left(\partial L_{2} \times \delta K \longrightarrow \partial L_{1} \times \delta K\right) \\
= & \operatorname{index}\left(\delta L_{1} \times K\right)=\operatorname{index}\left(\delta L_{1}\right) \cdot \operatorname{index}(K)
\end{aligned}
$$

(We have used the Novikov additivity property to compute index $\left(\delta L_{1} \times K \bigcup_{2^{s} \delta L_{1} \times \delta K} L_{1} \times \delta K\right)=\operatorname{index}\left(\delta L_{1} \times K\right)$.) On the other hand, 
index $\left(\delta L_{1}\right)$

$$
\begin{aligned}
& =\phi \rho_{8} \delta\left[P^{4 a+1}, f\right]-4\left\langle V S q^{1} V(\delta P) \cdot f^{*} \Phi(\tilde{\mathscr{K}}),[\delta P]\right\rangle \\
& =2^{3-s}\left\langle L(P) \cdot f^{*} \Phi \rho_{2^{s}}(\beta l),[P]\right\rangle-4\left\langle V S q^{1} V(\delta P) \cdot f^{*} \Phi(\tilde{\mathscr{K}}),[\delta P]\right\rangle,
\end{aligned}
$$

and, if $s=3$,

$$
\begin{aligned}
\operatorname{index}(K) & =\phi\left[Q^{4 b}, g\right]-4\left\langle V S q^{1} V(Q) \cdot g^{*} \Phi(\tilde{\mathscr{K}}),[Q]\right\rangle \\
& =\left\langle L(Q) \cdot g^{*} \Phi(l),[Q]\right\rangle-4\left\langle V S q^{1} V(Q) \cdot g^{*} \Phi(\tilde{\mathscr{K}}),[Q]\right\rangle \in Z / 8 .
\end{aligned}
$$

(If $s<3, \rho_{2^{s}}$ (index $\left.(K)\right)=\left\langle L(Q) \cdot g^{*} \Phi \rho_{2^{s}}(l),[Q]\right\rangle \in Z / 2^{s}$.) Using 9.7, the reader can check that, for $s \leqq 3$, this gives

$$
\begin{aligned}
& \phi \rho_{8} \delta\left(\left[P^{4 a+1}, f\right]\left[Q^{4 b}, g\right]\right) \\
& \quad=2^{3-s}\left\langle L(P) \cdot f^{*} \Phi \rho_{2^{s}}(\beta l),[P]\right\rangle\left\langle L(Q) \cdot g^{*} \Phi \rho_{2^{s}}(l),[Q]\right\rangle \in Z / 8 .
\end{aligned}
$$

By 9.9, this establishes 9.5 in Subcase 1.

Subcase 2. Let $L^{4 a+1}=f^{-1}(B S G) \subset P$, where $f: P^{4 a+1} \rightarrow M S G$ is transversal. Choose a $Z / 2$-partition of the $Z / 2$-manifold $g: Q^{4 b} \rightarrow M S G$, say $Q=U_{1} \cup U_{2}$ Thus $U_{1}$ and $U_{2}$ are $Z / 2$-manifolds with boundary. The notation is $\delta U_{i}=U_{i} \cap \delta Q, \partial U_{1}=U_{1} \cap U_{2}=\partial U_{2}$.

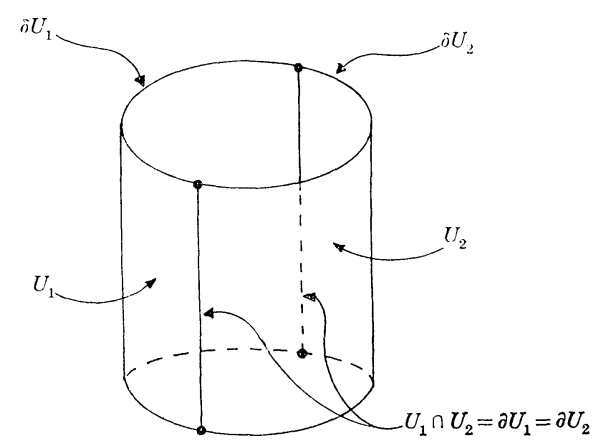

Note that $\delta Q=\delta U_{1} \cup \delta U_{2}$ will be a partition of $g: \delta Q \rightarrow M S G$.

We have transversal inverse images, $N_{1}=g^{-1}\left(M_{\gamma}\right) \cap U_{1}$ and $N_{2}=$ $g^{-1}\left(M_{\gamma}^{\prime}\right) \cap U_{2}, M_{\gamma}^{\prime} \subset M_{\gamma}$. $\quad N_{1}$ and $N_{2}$ are also $Z / 2$-manifold with boundary. The inclusion $\partial N_{2} \rightarrow \partial N_{1}$ is a normal map of $(4 b-2)$-dimenional $Z / 2$ manifolds and $s \delta\left[Q^{4 b}, g\right] \in Z / 2$ is the surgery obstruction $S \delta\left(\partial N_{2} \rightarrow \partial N_{1}\right) \in$ $Z / 2$.

Since $\delta(P \times Q)=\delta P \times Q \bigcup_{2 \delta \dot{\delta} \times \delta Q} P \times \delta Q$, the partition $(Q, \delta Q)=$ $\left(U_{1} \cup U_{2}, \delta U_{1} \cup \delta U_{2}\right)$ induces a partition of the closed manifold $\delta(P \times Q)$. Namely,

$$
\begin{aligned}
\delta(P \times Q)= & \left(\delta P \times U_{1} \bigcup_{2 \delta P \times \delta U_{1}} P \times \delta U_{1}\right) \underbrace{}_{\delta P \times\left(U_{1} \cap U_{2}\right) U P \times\left(\delta U_{1} \cap \hat{\rho} U_{2}\right)} \\
& \times\left(\delta P \times U_{2} \bigcup_{2 \delta P \times \delta U_{2}} P \times \delta U_{2}\right) \\
= & V_{1} \cup V_{2} .
\end{aligned}
$$


In the intersection, we get the "Massey product" normal map of closed $4 a+4 b-1$-dimensional $\mathrm{PD}$ spaces

$$
\delta L \times \partial N_{2} \bigcup_{2 \delta L \times \delta \partial N_{2}} L \times \delta \partial N_{2} \longrightarrow \delta L \times \partial N_{1} \bigcup_{2 \delta L \times \partial \partial N_{1}} L \times \delta \partial N_{1} .
$$

By Lemma 3.12, the $\theta$-invariant of this normal map is given by

$$
\begin{aligned}
4 d(L) \cdot & S \delta\left(\partial N_{2} \longrightarrow \partial N_{1}\right) \\
= & 4\left\langle V^{2}(P) \cdot f^{*} \Phi\left(V S q^{1} V\right)+V S q^{1} V(P) \cdot f^{*} \Phi\left(V^{2}\right),[P]\right\rangle \\
& \times\left\langle V^{2}(\delta Q) \cdot g^{*} \Phi(\mathscr{K}),[\delta Q]\right\rangle .
\end{aligned}
$$

In $V_{\imath}$, we have

$$
\delta L \times \partial N_{i} \bigcup_{2 \bar{\partial} L \times \delta \partial N_{i}} L \times \delta \partial N_{i}=\partial\left(\delta L \times N_{i} \bigcup_{2 \delta L \times \delta N_{i}} L \times \delta N_{i}\right), \quad i=1,2 .
$$

LEMMA 9.10.

$$
\begin{aligned}
& \operatorname{index}\left(\delta L \times N_{1} \bigcup_{2 \delta L \times \delta N_{1}} L \times \delta N_{1}\right) \\
& \quad+\operatorname{index}\left(\delta L \times N_{2} \bigcup_{2 \delta L \times \delta N_{2}} L \times \delta N_{2}\right) \equiv 0(\bmod 8)
\end{aligned}
$$

Assuming this lemma, we have shown that, if $f \times g: \delta(P \times Q) \rightarrow$ $M S G \wedge M S G$ is bordant to a transversal map $h: X \rightarrow M S G \wedge M S G$, and $Y=h^{-1}(B S G \times B S G) \subset X$, then

$$
\begin{aligned}
\text { index } & (Y) \\
= & 4\left\langle V^{2}(P) f^{*} \Phi\left(V S q^{1} V\right)+V S q^{1} V(P) \cdot f^{*} \Phi\left(V^{2}\right),[P]\right\rangle \\
& \times\left\langle V^{2}(\delta Q) \cdot g^{*} \Phi(\tilde{\mathscr{K}}),[\delta Q]\right\rangle \in Z / 8 .
\end{aligned}
$$

From 9.7 , we compute

$$
\begin{aligned}
& \phi \rho_{8} \delta\left(\left[P^{4 a+1}, f\right]\left[Q^{4 b}, g\right]\right) \\
&=4\left\langle V S q^{1}(\delta P) \cdot f^{*} \Phi(\tilde{\mathscr{C}}),[\delta P]\right\rangle\left\langle V^{2}(Q) \cdot g^{*} \Phi\left(V^{2}\right),[Q]\right\rangle \\
&+4\left\langle V^{2}(P) \cdot f^{*} \Phi\left(V S q^{1} V\right),[P]\right\rangle\left\langle V^{2}(\delta Q) \cdot g^{*} \Phi(\mathscr{K}),[\delta Q]\right\rangle \in Z / 8 .
\end{aligned}
$$

The reader can check that this implies 9.5 in Subcase 2. (It is necessary to use index $\left(\delta L^{4 a+1}\right)=0$, which implies $4\left\langle L(P) \cdot f^{*} \Phi \rho_{2}(\beta l),[P]\right\rangle=$ $\left.4\left\langle V S q^{1} V(\delta P) \cdot f^{*} \Phi(\widetilde{K}),[\delta P]\right\rangle.\right)$

Proof of Lemma 9.10. First, because of the computations above, this lemma is equivalent to 9.5 in our Subcase 2. If $g: Q^{4 b} \rightarrow M S G$ is transversal, we know 9.5 holds because of Subcase 1 . It thus suffices to consider a single non-transversal example $g: Q^{4 b} \rightarrow M S G$.

We can thus assume that the manifolds $N_{1}$ and $N_{2}$, which arise 
in the decomposition of $g: Q^{4 b} \rightarrow M S G$, have a very simple form. Specifically, we assume $N_{2}=D^{4} \times S^{3} \times I, \partial N_{2}=S^{3} \times S^{3} \times I, \delta N_{2}=$ $D^{4} \times S^{3}$, and $\delta \partial N_{2}=S^{3} \times S^{3}$ (the Kervaire 6-manifold), and we assume $N_{1}=D^{7} \times I, \partial N_{1}=S^{6} \times I, \delta N_{1}=D^{7}$, and $\delta \partial N_{1}=S^{6}$.

REMARK 9.11. The $Z / 2$-manifold $\partial N_{2}$ is obtained from $S^{3} \times S^{3} \times I$ via the diffeomorphism $T: S^{3} \times S^{3} \times\{0\} \stackrel{\sim}{\rightarrow} S^{3} \times S^{3} \times\{1\}, T(x, y, 0)=$ $(y, x, 0)$. If we identify $S^{3} \times S^{3}$ with $\partial\left(D^{4} \times S^{3}\right)$ in such a way that the diagonal $\triangle S^{3}=\{(x, x)\} \subset S^{3} \times S^{3}$ bound $D^{4} \times 1 \in D^{4} \times S^{3}$ (for example, via $S^{3} \times S^{3} \stackrel{\mu}{\rightarrow} S^{3} \times S^{3} \stackrel{\text { id }}{\rightarrow} D^{4} \times S^{3}$ where $\left.\mu(x, y)=\left(x, x^{-1} y\right)\right)$, then the orientation-reversing diffeomorphism $T: S^{3} \times S^{3} \stackrel{\sim}{\rightarrow} S^{3} \times S^{3}$ extends to $T: D^{4} \times S^{3} \rightarrow D^{4} \times S^{3}$. This explains how we regard $N_{2}=D^{4} \times S^{3} \times I$ as a $Z / 2$-manifold with boundary.

Similarly, $N_{1}=D^{7} \times I$ is a $Z / 2$-manifold with boundary, via the diffeomorphism $T^{\prime}: D^{7} \times\{0\} \stackrel{\sim}{\rightarrow} D^{7} \times\{1\}, T^{\prime}(x, 0)=(-x, 1)$.

With these assumptions, it is clear that index $\left(\delta L \times N_{i}\right)=0$, $i=1,2$. We will prove now that

$$
\begin{aligned}
& \text { index }\left(\delta L \times N_{i} \underset{2 o \delta \times \delta N_{i}}{\bigcup} L \times \delta N_{i}\right) \\
&=\operatorname{index}\left(\delta L \times N_{i}\right)+\operatorname{index}\left(L \times \delta N_{\imath}\right), i=1,2 .
\end{aligned}
$$

This will prove Lemma 9.10.

We use the result of Wall on non-additivity of the signature [24]. Let $Y=Y_{+} \cup Y_{-}$be $4 n$-manifolds with boundary. Let

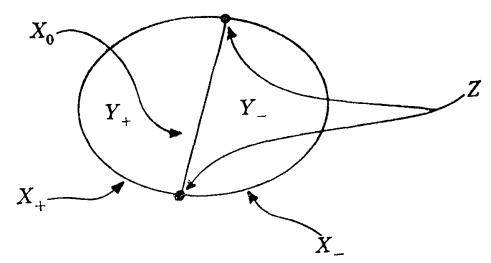

$X_{0}=Y_{+} \cap Y_{-}, X_{ \pm}=\partial Y_{ \pm}-X_{0}, Z=\partial X_{0}=\partial X_{ \pm}$. Let $A, B, C \subset H_{2 n-1}(Z, Q)$ be the kernels of the maps from $H_{2 n-1}(Z, Q)$ to $H_{2 n-1}\left(X_{+}, Q\right), H_{2 n-1}\left(X_{0}, Q\right)$, $H_{2 n-1}\left(X_{-}, Q\right)$, respectively. Then there is a non-singular symmetric quadratic form $\sigma: V \times V \rightarrow Q$, where $V=[A \cap(B+C)] /[A \cap B+A \cap C]$. Namely, if $a, a^{\prime} \in A \cap(B+C)$, write $a+b+c=0$ and $a^{\prime}+b^{\prime}+c^{\prime}=0$, where $b, b^{\prime} \in B$ and $c, c^{\prime} \in C$. Then define $\sigma\left(a, a^{\prime \prime}\right)=\left\langle a \cup b^{\prime},[Z]\right\rangle \in Q$. Moreover, index $(Y)=\operatorname{index}\left(Y_{+}\right)+\operatorname{index}\left(Y_{-}\right) \pm \operatorname{index}(V, \sigma)$.

In the situation of Lemma 9.10,

$$
\begin{aligned}
X_{+} & =\delta L \times \partial N_{i} \\
X_{0} & =2 \delta L \times \delta N_{i} \quad Z=2 \delta L \times \partial \delta N_{i} \\
X_{-} & =L \times \partial \delta N_{i} .
\end{aligned}
$$


Thus, if $i=2$,

$$
\begin{aligned}
& A=H_{*}(\delta L, Q) \otimes \operatorname{kernel}\left(H_{+}\left(\partial\left(S^{3} \times S^{3} \times I\right), Q\right) \longrightarrow H_{*}\left(S^{3} \times S^{3} \times I, Q\right)\right), \\
& B=H_{*}(\delta L, Q) \otimes \operatorname{kernel}\left(H_{*}\left(S^{3} \times S^{3} \times \partial I, Q\right) \longrightarrow H_{*}\left(D^{4} \times S^{3} \times \partial I, Q\right)\right), \\
& C=\operatorname{kernel}\left(H_{*}(\partial L, Q) \longrightarrow H_{*}(L, Q)\right) \otimes H_{*}\left(S^{3} \times S^{3}, Q\right) .
\end{aligned}
$$

Consider the automorphism of $H_{*}\left(S^{3} \times S^{3}, Q\right)$ defined by $T(x \otimes y)=$ $y \otimes x . \quad$ It is easy to see that $T$ induces automorphisms of $A, B$, and $C$, hence also an automorphism of $V=[A \cap(B+C)] /[A \cap B+A \cap C]$. Moreover, the reader can check that $\sigma\left(a, a^{\prime}\right)=-\sigma\left(T a, T a^{\prime}\right) \in Q$. It follows that index $(V, \sigma)=0$ in this case.

If $i=1$, it is easy to see directly that index $(V, \sigma)=0$, since $\partial N_{1}=S^{7} \times I, \delta N_{1}=D^{7} \times\{0,1\}$, and $\partial \delta N_{1}=S^{7}$ have such simple homology. This completes the proof of Lemma 9.10 and Subcase 2 .

Subcase 3. We have $\left[P^{4 a+1}, f\right]\left[Q^{4 b}, g\right]=\left[L^{6}\right]\left[S^{3}, \alpha\right]\left[Q^{4 b}, g\right]$.

We first compute the global transversality obstruction

$$
\begin{aligned}
s_{2}\left(\left[L^{6}\right]\left[S^{3}, \alpha\right]\left[Q^{4 b}, g\right]\right) & \text { by Theorem 2.4(iv) } \\
= & d\left(\delta L^{6}\right) s_{2}\left(\left[S^{3}, \alpha\right]\left[Q^{4 b}, g\right]\right) \\
= & d\left(\delta L^{6}\right)\left\langle V^{2}\left(S^{3} \times Q^{4 b}\right)(\alpha \times g)^{*} \Phi\left(\tilde{\mathscr{K}} \otimes V^{2}+V^{2} \otimes \mathscr{K}\right),\left[S^{3} \times Q^{4 b}\right]\right\rangle \\
= & d\left(\delta L^{6}\right)\left\langle\alpha^{*} \Phi(\tilde{\mathscr{K}}),\left[S^{3}\right]\right\rangle\left\langle V^{2}(Q) \cdot g^{*} \Phi\left(V^{2}\right),[Q]\right\rangle \\
= & \left\langle V S q^{1} V\left(\delta\left(L^{6} \times S^{3}\right)\right) \cdot\left(\alpha \pi_{2}\right)^{*} \Phi(\tilde{\mathscr{K}}),\left[\delta\left(L^{6} \times S^{3}\right)\right]\right\rangle \\
& \times\left\langle V^{2}(Q) \cdot g^{*} \Phi\left(V^{2}\right),[Q]\right\rangle .
\end{aligned}
$$

Using 9.7 and 9.8, this implies $\phi \rho_{8} \delta\left(\left[L^{6}\right]\left[S^{3}, \alpha\right]\left[Q^{4 b}, g\right]\right)=0$. On the other hand, one can easily check that the RHS of 9.5 also vanishes in this special case. This proves Subcases 3, hence completes the discussion of Case 5 .

Proof of Case 3. We wish to establish 9.5 for

$$
[X, h]=\left[M^{4 a+1}, f\right]\left[N^{4 b-1}, g\right], M, N
$$

$Z / 8$-manifolds. It suffices to consider the subcases: (1) $\left[N^{4 b-1}, g\right]=$ $\left[S^{3}, \alpha\right]$, and (2) $g: N^{4 b-1} \rightarrow M S G$ globally transversal. Let $\left[S^{3}, \alpha\right]=$ $\delta[\tilde{N}, \tilde{\alpha}], \tilde{N}$ a $Z / 8$-manifold. Then $\left[M^{4 a+1}, f\right]\left[S^{3}, \alpha\right]=\rho_{8} \delta\left(\left[M^{4 a+1}, f\right][\tilde{N}, \tilde{\alpha}]\right)+$ $\left[\delta M,\left.f\right|_{\delta M}\right][\tilde{N}, \tilde{\alpha}]$, and 9.5 holds in this case by our proofs of Cases 5 and 2 above. (Note that we did consider Bocksteins of $Z / 8$-products in Case 5.)

Now assume that $g: Q^{4 b-1} \rightarrow M S G$ is globally transversal. This implies that $f \times g: P^{4 a+1} \times Q^{4 b-1} \rightarrow M S G \wedge M S G$ is cobordant to a globally transversal map $h: X \rightarrow M S G \wedge M S G$. Let $k: R^{5} \rightarrow M S G$ be a $Z / 8$-manifold with $\phi \rho_{8} \delta\left[R^{5}, k\right]=1 \in Z / 8$. (For example, the Pontrja- 
gin-Thom construction applied to $C P(2)$ gives an element of $\pi_{4}(M S G)$ of order 8 . This can be used to produce $\left[R^{5}, k\right]$.) Consider the product $\left(\left[P^{4 a+1}, f\right]\left[Q^{4 b-1}, g\right]\right)\left(\left[R^{5}, k\right]\right)$, which is cobordant to $\left[X^{4 a+4 b}, h\right]\left[R^{5}, k\right]$. In Subcase 1 of Case 5, we proved that

$$
\begin{aligned}
\phi \rho_{8} \delta\left(\left[R^{5}, k\right]\left[X^{4 a+4 b}, h\right]\right) & =\phi \rho_{8} \delta\left[R^{5}, k\right] \cdot \phi\left[X^{4 a+4 b}, h\right] \\
& \equiv \phi\left[X^{4 a+4 b}, h\right] \in Z / 8 .
\end{aligned}
$$

On the other hand, let us compute $\phi \rho_{8} \delta$ by associating differently. We see

$$
\phi \rho_{8} \delta\left(\left(\left[Q^{4 b-1}, g\right]\right)\left(\left[P^{4 a+1}, f\right]\left[R^{5}, k\right]\right)\right)=0,
$$

from Subcase 1 of Case 4 . Thus we have proved $\phi\left(\left[P^{4 a+1}, f\right]\left[Q^{4 b-1}, g\right]\right)=0$ if $g: Q^{4 b-1} \rightarrow M S G$ is globally transversal. It is easy to check that the RHS of 9.5 also vanishes in this case.

This completes the proof of all five cases of 9.5 , hence we have established 9.1.

\section{CHAPTER IV}

10. Homotopy-theoretic obstructions to global transversality. Let $\xi \rightarrow B \xi$ be a spherical fibration, with Thom spectrum $T \xi$. In Chapter I, §3, we defined certain homomorphisms (assuming $\pi_{1}(B \xi)=0$ and $\xi$ oriented)

$$
\begin{aligned}
& s_{n}: \widetilde{\Omega}_{4^{*}+1}^{P L}(T \xi, Z / n) \longrightarrow P_{*} \otimes Z / n, \\
& \widetilde{s}: \widetilde{\eta}_{2^{*+1}}^{P L}(T \xi) \longrightarrow Z / 2,
\end{aligned}
$$

using the obstruction theory to Poincaré transversality developed in I, $\S 1$ and $\S 2$. In this chapter, we will give purely homotopy-theoretic definitions of $s_{n}$ and $\widetilde{s}$. Since $\widetilde{s}$ determines $\widetilde{\mathscr{K}} \in H^{*-1}(B S G, Z / 2)$ and since $s_{2^{\infty}}=\lim s_{2^{s}}$ determines $\tilde{\mathscr{L}} \in H^{4^{*+1}}\left(B S G, Z_{(2)}\right)$, our homotopytheoretic definitions of $\widetilde{s}$ and $s_{2^{s}}$ give, in some sense, a homotopytheoretic definition of the transversality obstructions $\tilde{\mathscr{K}}$ and $\tilde{\mathscr{L}}$.

Among the consequences of the new definition are these. First, we can formulate homotopy-theoretic conditions which imply that $\tilde{\mathscr{K}}$ and $\tilde{\mathscr{L}}$ vanish, and therefore, by Theorem 6.2 , we can formulate conditions that imply a map $f: X \rightarrow B S G_{(2)}$ lifts to $f_{T O P}: X \rightarrow B S T O P_{(2)}$. Secondly, we prove that, if $[M, f] \in \widetilde{\eta}_{* *}^{P L}(T \xi)$ and if $f: M \rightarrow T \xi$ is globally transversal then $s([M, f])=0$. This proves the converse of Theorem 3.4 in the one case we did not deal with in $\S 3$ (see proof of Theorem 3.8). It also follows that $\widetilde{s}: \tilde{\eta}_{2^{*}+1}^{P L}(T \xi) \rightarrow Z / 2$ is independent of the "good" cover $\left\{V_{\alpha}\right\}$ of $T \xi$, which occurs implicitly in the original definition of $\widetilde{s}$ in $\S 3$. Thirdly, based on the new definition of $\widetilde{s}$, we 
outline a purely homotopic-theoretic proof of the Whitney sum formula for the class $\tilde{\mathscr{K}}$, namely, $\tilde{\mathscr{K}}(\xi \times \eta)=\tilde{\mathscr{K}}(\xi) \otimes V^{2}(\eta)+V^{2}(\xi) \otimes \tilde{\mathscr{K}}(\eta)$. Finally, the new definition of $\widetilde{s}$ applies to any spherical fiber space $\xi$, and the new definition of $s_{n}$ applies to any oriented $\xi$; that is, we drop the assumptions on $\pi_{1}(B \xi)$. (However, $\widetilde{s}$ and $s_{n}$ are only partial obstructions to global transversality in the nonsimply connected case.)

We will first define $\widetilde{s}: \widetilde{\eta}_{2^{*}-1}^{P L}(T \xi) \rightarrow Z / 2$. The main idea is due to W. Browder ([3]). The homology theory $\eta_{*}^{P_{L}}$ is represented by the spectrum $M P L$ (the Thom spectrum of the universal $P L$-bundle $\gamma \rightarrow$ $B P L)$. Thus, there is an isomorphism $p: \tilde{\eta}_{*}^{P L}(T \xi) \stackrel{\sim}{\rightarrow} \pi_{*}(T \xi \wedge M P L)$. $T \xi \wedge M P L$ is the Thom spectrum of the Whitney sum $\xi \times \gamma \rightarrow B \xi \times$ $B P L$, and the isomorphism $p$ is easily defined directly as a PontrjaginThom construction. Following Browder, we will define, for any spherical fibre space $\eta$, a homomorphism $\phi_{\eta}: \pi_{2^{*-1}}(T \eta) \rightarrow Z / 2$. Then we define $\widetilde{s}_{\xi}: \tilde{\eta}_{2^{*}-1}^{P L}(T \xi) \rightarrow Z / 2$ as the composition

$$
\hat{s}_{\xi}=\phi_{\xi \times r^{\circ}} p: \tilde{\eta}_{2^{*}-1}^{P L}(T \xi) \longrightarrow \pi_{2^{*}-1}(T \xi \wedge M P L) \longrightarrow Z / 2 .
$$

To define $\phi_{\eta}: \pi_{2 n-1}(T \eta) \rightarrow Z / 2$, we recall some results of [3]. Let $K(Z / 2, n-1) \rightarrow B \eta\left\langle v_{n}\right\rangle \rightarrow B \eta$ be the fibration which kills the $\mathrm{Wu}$ class $v_{n}(\eta) \in H^{n}(B \eta, Z / 2)$. Let $T \eta\left\langle v_{n}\right\rangle$ be the Thom spectrum of the induced bundle over $B \eta\left\langle v_{n}\right\rangle$. Roughly, if $\alpha: S^{2 n-1} \rightarrow T \eta, \phi_{\eta}(\alpha)$ will be the obstruction to lifting $\alpha$ to $\tilde{\alpha}: S^{2 n-1} \rightarrow T \eta\left\langle v_{n}\right\rangle$. We regard $B \eta\left\langle v_{n}\right\rangle$ as a subspace of $B \eta$. Let $v \in H^{n}\left(B \eta, B \eta\left\langle v_{n}\right\rangle, Z / 2\right)=Z / 2$ be the generator. The composition

$$
B \eta, B \eta\left\langle v_{n}\right\rangle \stackrel{\Delta}{\longrightarrow} B \eta \times\left(B \eta, B \eta\left\langle v_{n}\right\rangle\right) \stackrel{1 \times v}{\longrightarrow} B \eta \times(K(Z / 2, n), e),
$$

where $\Delta$ is the diagonal and $e \in K(Z / 2, n)$ is a basepoint, induces a map on the Thom space level

$$
T \eta \mid T \eta\left\langle v_{n}\right\rangle \stackrel{f_{\eta}}{\longrightarrow} T \eta \wedge K(Z / 2, n)^{+} / T \eta=T \eta \wedge K(Z / 2, n) .
$$

Theorem 10.1 (Browder). (i) $f_{\eta}^{*}: H^{i}(T \eta \wedge K(Z / 2, n), Z / 2) \rightarrow$ $H^{2}\left(T \eta / T \eta\left\langle v_{n}\right\rangle, Z / 2\right)$ is an isomorphism if $i<2 n$, and a surjection with kernel $=Z / 2$ if $i=2 n$. The generator of kernel $\left(f_{\eta}^{*}\right)$ in dimension $2 n$ is the element $v_{n}(\eta) \cdot U_{\eta} \otimes \iota_{n}+U_{\eta} \otimes \iota_{n}^{2}=\sum_{i=1}^{n} S q^{i}\left(v_{n-1}(\eta) U_{\eta} \otimes \iota_{n}\right) \epsilon$ $H^{2 n}(T \eta \wedge K(Z / 2, n), Z / 2)$, where $U_{n} \in H^{*}(T \eta, Z / 2)$ is the Thom class, and $\iota_{n} \in H^{*}(K(Z / 2, n), Z / 2)$ is the fundamental class.

(ii) Through dimension $2 n, T \eta \wedge K(Z / 2, n)$ is a product of $K(Z / 2)$-spectra. Specifically, through dimension $2 n$,

$$
H^{*}(T \eta \wedge K(Z / 2, n), Z / 2)
$$

is a free module over the Steenrod algebra $\mathscr{A}_{2}$, with basis $\left\{y U_{\eta} \otimes \iota_{n}\right\}$, where $\{y\} \in H^{*}(B \eta, Z / 2)$ is a $Z / 2$-basis. 
(iii) Let $y \in H_{i}(B \eta, Z / 2)$. Then $\rho^{*} f_{\eta}^{*}\left(y U_{\eta} \otimes c_{n}\right)=y \cdot v_{n} \cdot U_{\eta} \in H^{n+i}(T \eta$, $Z / 2)$, where $\rho: T \eta \rightarrow T \eta \mid T \eta\left\langle v_{n}\right\rangle$ is the obvious projection.

Proof. See [3] and [16].

It follows from 10.1(i) and (ii) that, through dimension $2 n-1$, $T \eta / T \eta\left\langle v_{n}\right\rangle$ is a 2 -stage Postnikov system, with $k$-invariant

$$
X=\sum_{i=1}^{n} S q^{i}\left(v_{n-i} \cdot U_{\eta} \otimes \iota_{n}\right) \in H^{2 n}(T \eta \wedge K(Z / 2, n), Z / 2) .
$$

In particular, there is an exact sequence

$$
0 \longrightarrow Z / 2 \longrightarrow \pi_{2 n-1}\left(T \eta / T \eta\left\langle v_{n}\right\rangle\right) \stackrel{h}{\longrightarrow} H_{2 n-1}\left(T \eta / T \eta\left\langle v_{n}\right\rangle, Z / 2\right),
$$

where $h$ is the Hurewicz homomorphism. Moreover, if $\beta: S^{2 n-1} \rightarrow$ $T \eta / T \eta\left\langle v_{n}\right\rangle$ represents an element of kernel $(h)=Z / 2$, then $\beta$ is evaluated as follows. Let $x_{2 n-i}=f_{\eta}^{*}\left(v_{n-i} U_{\eta} \otimes \iota_{n}\right) \in H^{2 n-1}\left(T \eta / T \eta\left\langle v_{n}\right\rangle, Z / 2\right)$, so that $\sum_{i=1}^{n} S q^{i}\left(x_{2 n-i}\right)=0$. Let $\bar{x}_{2 n-i} \in H^{2 n-1}\left(T \eta / T \eta\left\langle v_{n}\right\rangle \mathbf{U}_{\beta} e^{2 n}, Z / 2\right)$ be the unique element, $0<i<n$, which restricts to

$$
x_{2 n-i} \in H^{2 n-i}\left(T \eta / T \eta\left\langle v_{n}\right\rangle, Z / 2\right) .
$$

Then $\beta \in Z / 2$ is computed as the functional operation

$$
\begin{aligned}
& \sum_{i=1}^{n} S q^{i}\left(\bar{x}_{2 n-1}\right) \in Z / 2 \\
& \quad=\text { image }\left(H^{2 n-1}\left(S^{2 n-1}, Z / 2\right) \stackrel{\delta}{\longrightarrow} H^{2 n}\left(T \eta / T \eta\left\langle v_{n}\right\rangle \bigcup_{\beta} e^{2 n}, Z / 2\right)\right) .
\end{aligned}
$$

From 10.1, we also deduce the following.

Corollary 10.2. The composition $\rho_{*} h: \pi_{j}(T \eta) \rightarrow \pi_{j}\left(T \eta / T \eta\left\langle v_{n}\right\rangle\right) \rightarrow$ $H_{j}\left(T \eta / T \eta\left\langle v_{n}\right\rangle, Z / 2\right)$ is zero, $j<2 n$. Hence

$$
\rho_{*} \pi_{2 n-1}(T \eta) \subset Z / 2 \subset \pi_{2 n-1}\left(T \eta / T \eta\left\langle v_{n}\right\rangle\right) .
$$

Proof. By 10.1(i), (ii), it suffices to show that, for $y \in H^{i}(B \eta, Z / 2)$, $i<n$, the element $\rho^{*} f_{\eta}^{*}\left(y U \otimes \iota_{n}\right) \in H^{n+i}(T \eta, Z / 2)$ is decomposable over the Steenrod algebra. But by 10.1(iii), and since $S q^{n}(y)=0$,

$$
\begin{aligned}
\rho^{*} f_{r_{i}}^{*}\left(y U_{\eta} \otimes c_{n}\right) & =y \cdot v_{n} \cdot U_{\eta}=y \cdot \chi\left(S q^{n}\right) U_{\eta} \\
& =\sum_{i=1}^{n} S q^{i}\left(y \cdot \chi\left(S q^{n-i}\right) U_{\eta}\right)+S q^{n}(y) \cdot U_{\eta} \\
& =\sum_{i=1}^{n} S q^{i}\left(y \cdot v_{n-i} U_{\eta}\right) .
\end{aligned}
$$

(We have used the identity $S q(a \cdot \chi(S q) b)=S q(a)(b)$. 
We now define $\phi_{n}: \pi_{2 n-1}(T \eta) \rightarrow Z / 2$ to be

$$
\rho_{*}: \pi_{2 n-1}(T \eta) \longrightarrow \pi_{2 n-1}\left(T \eta / T \eta\left\langle v_{n}\right\rangle\right),
$$

with image $\left(\rho_{*}\right) \subset Z / 2=$ kernel $(h)$. Thus, given $\alpha: S^{2 n-1} \rightarrow T \eta, \phi_{\eta}(\alpha) \in$ $Z / 2$ is computed as the functional operation

$$
\sum_{i=1}^{n} S q^{i}\left(\bar{x}_{2 n-i}\right) \in Z / 2 \subset H^{2 n}\left(T \eta / T \eta\left\langle v_{n}\right\rangle \bigcup_{\rho \alpha} e^{2 n}, Z / 2\right) .
$$

REMARK 10.3. It is easy to see that $\phi_{\eta}$ commutes with bundle maps. That is, if $\hat{f}: T \eta \rightarrow T \eta^{\prime}$ is induced by a bundle map $f: \eta \rightarrow \eta^{\prime}$, then $\phi_{\eta}=\phi_{\eta} \circ \hat{f}_{*}: \pi_{2 n-1}(T \eta) \rightarrow Z / 2$. It follows that, if $\hat{g}: T \xi \rightarrow T \xi^{\prime}$ is induced by a bundle map $g: \xi \rightarrow \xi^{\prime}$, then $\widehat{s}_{\xi}=\hat{s}_{\xi^{\prime}} \circ \hat{g}_{*}: \eta_{\eta_{*}}^{P L}(T \xi) \rightarrow Z / 2$.

REMARK 10.4. Suppose that $\eta$ is oriented, and that $n$ is odd. Then $v_{n}(\eta)=0$, hence $\pi_{2 n-1}\left(T \eta\left\langle v_{n}\right\rangle\right) \rightarrow \pi_{2 n-1}(T \eta)$ is clearly surjective. Thus $\phi_{\eta} \equiv 0: \pi_{4 k+1}(T \eta) \rightarrow Z / 2$. Since $\widetilde{\Omega}_{*}^{P L}(T \xi)=\pi_{*}(T \xi \wedge M S P L)$, we see that, if $\xi$ is oriented, $\hat{s} \equiv 0: \Omega_{4 k+1}^{P L} \rightarrow Z / 2$. With somewhat more work, one can prove that $\hat{s} \equiv 0: \widetilde{\Omega}_{4 k+1}^{P L}(T \xi, Z / 2) \rightarrow Z / 2$. In fact, this will follow below when we prove that $\hat{s} \equiv \widetilde{s}: \eta_{2^{*}-1}^{P L}(T \xi) \rightarrow Z / 2$. The fact that $\widetilde{s} \equiv 0: \widetilde{\Omega}_{4 k+1}^{P L}(T \xi, Z / 2) \rightarrow Z / 2$ follows from the result of Wall ([24)] that the Kervaire obstruction is zero for a normal map of $4 k$-dimensional $Z / 2$-manifolds.

We now begin the proof that $\hat{s} \equiv \widetilde{s}: \eta_{*}^{P L}(M S G) \rightarrow Z / 2$. It is easy to see that it suffices to prove $\operatorname{kernel}(\widetilde{s}) \subset \operatorname{kernel}(\hat{s})$ and $\hat{s} \not \equiv 0$ : $\eta_{2 n+1}^{P L}(M S G) \rightarrow Z / 2, n \geqq 1$.

TheOREM 10.5 (Browder). If $\alpha: S^{q+2 n-1} \rightarrow T \eta^{q}$ is Poincaré-globally transversal, then $\phi_{\eta}(\alpha)=0$.

Proof. We need to prove that $\alpha$ lifts to $\tilde{\alpha}: S^{q+2 n-1} \rightarrow T \eta^{q}\left\langle v_{n}\right\rangle$. By assumption, $\alpha$ factors through the Thom space of the normal bundle of a PD space $L^{2 n-1}, \alpha: S^{2 n-1} \rightarrow T\left(\nu_{L}^{q}\right) \rightarrow T\left(\eta^{q}\right)$. Since $v_{n}\left(\nu_{L}^{q}\right)=$ $v_{n}\left(L^{2 n-1}\right)=0$, we see that $\alpha$ lifts to $\tilde{\alpha}: S^{2 n-1} \rightarrow T\left(\nu_{L}^{q}\right) \rightarrow T \eta^{q}\left\langle v_{n}\right\rangle$, as desired.

As a corollary, we see that, if $f: M^{q+2 n-1} \rightarrow T \xi^{q}$ is Poincaré transversal, then $\hat{s}[M, f]=\phi_{\xi \times r}(p[M, f])=0$, where $p: \widetilde{\eta}_{q+2 n+1}^{P L}\left(T \xi^{q}\right) \stackrel{\sim}{\rightarrow}$ $\pi_{3 q+2 n-1}\left(T \xi^{q} \wedge M P L(2 q)\right)$ is the Pontrjagin-Thom map. For, if $f: M \rightarrow T \xi$ is transversal, then $p[M, f]: S \rightarrow T \xi \wedge M P L$ is transversal. In fact, the transversal inverse images $f^{-1}\left(B \xi^{q}\right) \subset M^{q+2 n-1}$ and

$$
(p[M, f])^{-1}\left(B \xi^{q} \times B P L(2 q)\right) \subset S^{3 q+2 n-1}
$$

can be identified as $\mathrm{PD}$ spaces, since $p[M, f]$ is defined in terms of 
an embedding $M^{q+2 n-1} \subset S^{3 q+2 n-1}$. By Theorem 3.4, we conclude that, if $\widetilde{s}[M, f]=0$, then $\hat{s}[M, f]=0$; that is, $\operatorname{kernel}(\widetilde{s}) \subset \operatorname{kernel}(\hat{s})$.

THEOREM 10.6. If $n \geqq 1, \hat{s}: \eta_{2 n+1}^{P L}(M S G) \rightarrow Z / 2$ is nonzero.

Proof. This is essentially due to Browder. Here is the idea. First, we will show that $\pi_{3}(M S G)=Z / 2$, and that $\phi: \pi_{3}(M S G) \rightarrow Z / 2$ and is nonzero. It is easy to see that the diagram

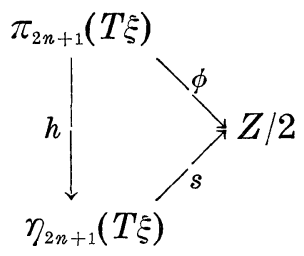

commutes, hence 10.6 is true if $n=1$. Next, there is the natural action of $\eta_{*}^{P L}(p t)$ on $\eta_{*}^{P L}(T \xi)$. We will show that, if $[M, f] \in \eta_{*}^{P L}(T \xi)$ $[N] \in \eta_{*}^{P L}(p t)$, then

$$
\hat{s}([M, f][N])=\hat{s}([M, f]) \cdot\left\langle V^{2}(N),[N]\right\rangle \in Z / 2 .
$$

Thus we obtain 10.6 for $n>1$ by taking $[M, f]$ to be the generator of $\pi_{3}(M S G)=Z / 2$ and $[N]$ to be $[R P(2 n)]$.

To prove that $\pi_{3}(M S G)=Z / 2$, we recall $([14])$

$$
H^{i}(B S G, Z / 2)= \begin{cases}Z / 2 & \text { if } i=0, \\ 0 & \text { if } i=1, \\ Z / 2 & \text { if } i=2 ; \text { generator }=w_{2}=v_{2}, \\ Z / 2 \oplus Z / 2 & \text { if } i=3 \text {; generator }=w_{3} \text { and } e_{3} .\end{cases}
$$

Moreover, $S q^{1}\left(e_{3}\right) \neq 0 \in H^{4}(B S G, Z / 2)$. (Here, $e_{3} \in H^{3}(B S G, Z / 2)$ is the first exotic class ([7].) This computes $H^{i}(M S G, Z / 2), i \leqq 3$, and it is easy to deduce that

$$
\pi_{i}(M S G)= \begin{cases}Z & \text { if } \quad i=0 \\ 0 & \text { if } i=1 \\ 0 & \text { if } i=2 \\ Z / 2 & \text { if } i=3\end{cases}
$$

(One knows from [17] that $M S G$ is a product of $K(\pi)$-spectra, and it is easy to prove there is no odd torsion in this range.)

To see now that $\phi: \pi_{3}(M S G) \rightarrow Z / 2$ is nonzero, it is obviously sufficient to prove $\pi_{3}\left(M S G\left\langle v_{2}\right\rangle\right)=0$. From the Serre spectral sequence for $K(Z / 2,1) \rightarrow B S G\left\langle v_{2}\right\rangle \rightarrow B S G$, one computes 


$$
H^{i}\left(B S G\left\langle v_{2}\right\rangle, Z / 2\right)= \begin{cases}Z / 2 & \text { if } i=0, \\ 0 & \text { if } i=1, \\ 0 & \text { if } i=2, \\ Z / 2 & \text { if } i=3 ; \text { generator }=\pi^{*}\left(e_{3}\right) .\end{cases}
$$

Moreover, $S q^{1}\left(\pi^{*} e_{3}\right) \neq 0 \in H^{4}\left(B S G\left\langle v_{2}\right\rangle, Z / 2\right)$. Consider now the Postnikov system for $M S G\left\langle v_{2}\right\rangle$. It is clear that it must begin as

$$
K(Z, 0) \bigcup_{S q^{2} \iota_{0}} K(Z / 2,1) \text {. }
$$

Now

$$
H^{i}\left(K(Z, 0) \bigcup_{S q^{2} \epsilon_{0}} K(Z / 2,1), Z / 2\right)=\left\{\begin{array}{lll}
Z / 2 & \text { if } i=0, \\
0 & \text { if } i=1, \\
0 & \text { if } i=2, \\
Z / 2 & \text { if } i=3,
\end{array}\right.
$$

Moreover, the generator in dimension 3 restricts to

$$
S q^{2} \iota_{1} \in H^{3}(K(Z / 2,1), Z / 2) \text {. }
$$

From the well-known relation between $\pi^{*} e_{3} \cdot U$ and the secondary operation corresponding to the relation $S q^{2} S q^{2} \iota_{0}=0 \in H^{4}(K(Z, 0), Z / 2)$ ([7]), we see that $M S G\langle v\rangle \rightarrow K(Z, 0) \bigcup_{S q 2 \iota_{0}} K(Z / 2,1)$ induces an isomorphism in cohomology through $H^{3}$ and an injection in $H^{4}$. Thus $\pi_{3}\left(M S G\left\langle v_{2}\right\rangle\right)=0$.

We will deduce 10.7 from the following product formula for $\phi: \pi_{*}(T \eta) \rightarrow Z / 2$.

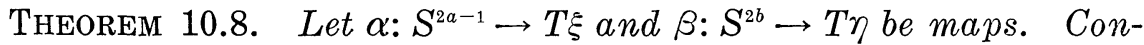
sider $\alpha \wedge \beta: S^{2 a+2 b-1}=S^{2 a-1} \wedge S^{2 b} \rightarrow T \xi \wedge T \eta$. Then

$$
\dot{\phi}_{\xi \times \eta}(\alpha \wedge \beta)=\dot{\phi}_{\xi}(\alpha) \cdot\left\langle\beta^{*} \Phi\left(V^{2}\right),\left[S^{2 b}\right]\right\rangle \in Z / 2 .
$$

To derive 10.7 from 10.8 , we have isomorphisms $y: \tilde{\eta}_{*}^{P L}(T \xi) \stackrel{\sim}{\rightarrow}$ $\pi_{*}(T \xi \wedge M P L)$ and $p: \eta_{*}^{P L}(p t) \stackrel{\sim}{\rightarrow} \pi_{*}(M P L)$. $M P L$ is a ring spectrum with multiplication $\mu: M P L \wedge M P L \rightarrow M P L$ induced by Whitney sum. The module structure of $\eta_{*}^{P L}(T \xi)$ over $\eta_{*}^{P L}(p t)$ is then induced by $1 \wedge \mu: T \xi \wedge M P L \wedge M P L \rightarrow T \xi \wedge M P L$, and we have

$$
\begin{aligned}
\hat{s}([M, f][N] & =\phi_{\xi \times r}\left((1 \wedge \mu)_{*}(p[M, f] \wedge p[N])\right) \\
& =\phi_{\xi \times r \times r}(p[M, f] \wedge p[N]) \quad \text { by Remark } 10.3 \\
& =\phi_{\xi \times r}(p[M, f]) \cdot\left\langle(p[N])^{*} \Phi\left(V^{2}\right)\left[S^{\mathrm{dim} N}\right]\right) \text { by } 10.8 \\
& =\hat{s}([M, f]) \cdot\left\langle V^{2}(N),[N]\right\rangle .
\end{aligned}
$$

The product formula 10.7 implies that there is a graded class 
$\widetilde{\mathscr{K}}(\xi) \in H^{2^{*-1}}(B \xi, Z / 2)$ such that, for any $f: M \rightarrow T \xi, \hat{s}([M, f])=$ $\left\langle V^{2}(M) \cdot f^{*}(\Phi \tilde{\mathscr{K}}(\xi)),[M]\right\rangle \in Z / 2$. The argument just above deriving 10.7 from 10.8 generalizes easily to give the following. Let $f$ : $M^{q+2 a-1} \rightarrow T \xi^{q}, g: N^{q+2 b} \rightarrow T \eta^{q}$ be maps. Then $\hat{s}([M \times N, f \wedge g])=$ $\hat{s}([M, f]) \cdot\left\langle g^{*} \Phi\left(V^{2}\right),[N]\right\rangle \in Z / 2$, where $f \wedge g: M \times N \rightarrow T \xi \times T \eta \rightarrow T \xi \wedge T \eta$. A simple argument then shows that the characteristic class $\tilde{\mathscr{K}}$ satisfies the Whitney sum formula

$$
\begin{aligned}
& \tilde{\mathscr{K}}(\xi \times \eta) \\
& \quad=\tilde{\mathscr{K}}(\xi) \otimes V^{2}(\eta)+V^{2}(\xi) \otimes \tilde{\mathscr{K}}(\eta) \in H^{2^{*-1}}(B \xi \times B \eta Z /, 2) .
\end{aligned}
$$

If $\pi_{1}(B \xi)=0$, then $\hat{s}=\widetilde{s}: \eta_{*}(T \xi) \rightarrow Z / 2$. Thus $\tilde{\mathscr{K}}(\xi)$ is the class $\widetilde{\mathscr{K}}_{4^{*}-1} \in H^{4^{*-1}}(B \xi, Z / 2)$ defined in II, $\S 5$. We have thus used 10.8 to prove a Whitney sum formula for $\tilde{\mathscr{K}}_{4^{*}-1}$. In particular, consider $\tilde{\mathscr{K}}_{4^{*}-1} \in H^{4^{*-1}}(B S G, Z / 2)$.

THEOREM 10.10. $\tilde{\mathscr{K}}_{4^{*}-1}=V^{2}\left(\sum_{i \geqq 2} e_{2^{i}-1}\right) \in H^{4^{*}-1}(B S G, Z / 2)$, where $e_{2^{i}-1} \in H^{2^{i}-1}(B S G, Z / 2)$ is the unique primitive class with $\sigma\left(e_{2^{i}-1}\right)=$ $i^{*}\left(\mathscr{C}_{2^{i}-2}\right) \in H^{2^{i-2}}(S G, Z / 2), \mathscr{K}_{2^{i}-2} \in H^{2^{i-2}}(G / T O P, Z / 2)$ the surgery obstruction class.

Proof. From 10.9, $\widetilde{\mathscr{K}}_{4^{*}-1} \cdot(V)^{-2} \in H^{4^{*}-1}(B S G, Z / 2)$ is primitive. On the other hand, $\sigma\left(\widetilde{\mathscr{K}}_{4^{*}-1} \cdot(V)^{-2}\right)=\sigma\left(\widetilde{\mathscr{K}}_{4^{*}-1}\right)=i^{*}\left(\mathscr{K}_{4^{*}-2}\right)$ by 7.2. In [4], it is shown that $i^{*}\left(\mathscr{K}_{4 n-2}\right)=0$ if $4 n \neq 2^{i}$. We also have $j^{*}\left(\mathscr{K}_{4^{*-1}}\right) \equiv$ $0 \in H^{4^{*-1}}(B S O, Z / 2), j: B S O \rightarrow B S G .10 .10$ now follows from the fact the primitives in $H^{*}(B S G, Z / 2)$ which vanish in $H^{*}(B S O, Z / 2)$ inject under $\sigma$ into $H^{*}(S G, Z / 2)([14])$.

We now return to the proof of Theorem 10.8.

Proof of 10.8. Step 1. It suffices to assume $v_{2}(\xi)=0, i>a$, and $v_{j}(\eta)=0, j>b$, hence $v_{a+b}(\xi \times \eta)=v_{a}(\xi) \otimes V_{b}(\eta)$. For let

$$
\prod_{i \geq 0} K(Z / 2, a+i) \longrightarrow B \hat{\xi} \longrightarrow B \xi
$$

be the fibration which kills $v_{a+i+1}(\xi), i \geqq 0$. Similarly, define $B \hat{\eta}$ by killing $v_{b+j+1}(\eta), j \geqq 0$. Let $T \hat{\xi}$ and $T \hat{\eta}$ be the Thom spaces over $B \hat{\xi}$ and $B \hat{\eta}$, respectively. It follows from 10.2 that $\pi_{2 a-1}(T \hat{\xi}) \rightarrow \pi_{2 a-1}(T \xi)$ and $\pi_{2 b}(T \hat{\eta}) \rightarrow \pi_{2 b}(T \eta)$ are surjective. If $\hat{\alpha}: S^{2 a-1} \rightarrow T \hat{\xi}$ and $\hat{\beta}: S^{2 b} \rightarrow T \hat{\eta}$ lift $\alpha$ and $\beta$, respectively, then, by Remark 10.3, $\dot{\phi}_{\xi \times \eta}(\alpha \wedge \beta)=\phi_{\hat{\xi} \times \hat{\eta}}$ $(\hat{\alpha} \wedge \widehat{\beta}) \in Z / 2$, and also $\phi_{\xi}(\alpha) \cdot\left\langle\beta^{*} \Phi\left(V^{2}\right),\left[S^{2 b}\right]\right\rangle=\phi_{\hat{\hat{\xi}}}(\hat{\alpha})\left\langle\widehat{\beta}^{*} \Phi\left(V^{2}\right),\left[S^{2 b}\right]\right\rangle \epsilon$ $Z / 2$.

Step 2. There is a commutative diagram 


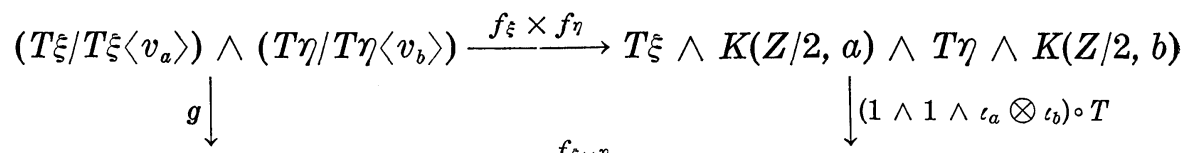

$T \xi \wedge T \eta / T \xi \wedge T \eta\left\langle v_{a} \otimes v_{b}\right\rangle \stackrel{f_{\xi \times \eta}}{\longrightarrow} T \xi \wedge T \eta \wedge K(Z / 2, a+b)$

where the map $T$ switches the factors $K(Z / 2, a)$ and $T \eta$. Moreover, $g$ is constructed so that

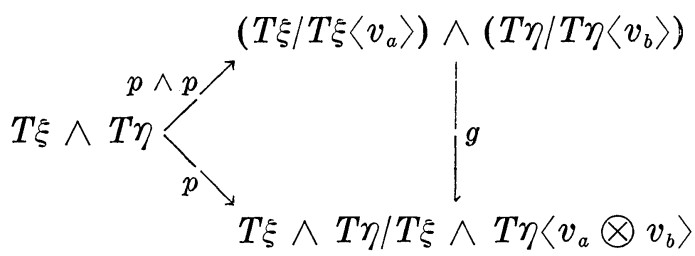

commutes.

To see this, one constructs on the base spaces a commutative diagram of maps of pairs

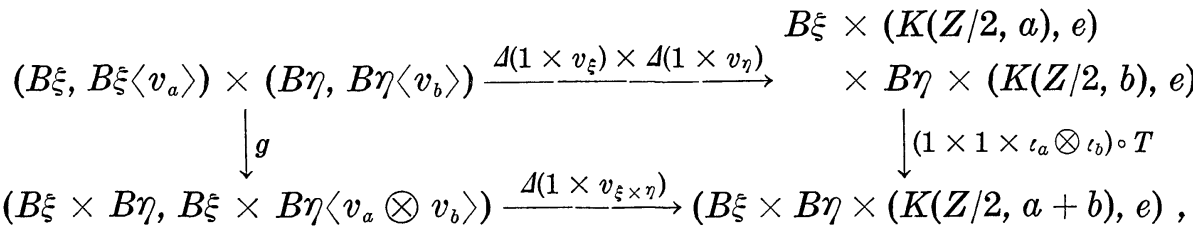

where $v_{\xi} \in H^{a}\left(B \xi, B \xi\left\langle v_{a}\right\rangle, Z / 2\right), v_{\eta} \in H^{b}\left(B \eta, B \eta\left\langle v_{b}\right\rangle, Z / 2\right)$, and $v_{\xi \times \eta} \in$ $H^{a+b}\left(B \xi \times B \eta, B \xi \times B \eta\left\langle v_{a} \otimes v_{b}\right\rangle, Z / 2\right)$ are the unique nonzero elements.

Step 3. The functional operation

$$
\begin{aligned}
& \dot{\phi}_{\xi \times \eta}(\alpha \wedge \beta) \\
& \quad=\sum_{1=1}^{a+b} S q_{1}\left(\bar{x}_{2 a+2 b-i}(\xi \times \eta)\right) \in H^{2 a+2 b}\left(T \xi \wedge T \eta / T \xi \wedge T \eta\left\langle v_{a} \otimes v_{b}\right\rangle \bigcup_{\rho(\alpha \wedge \beta)} e^{2 a+2 b}, Z / 2\right)
\end{aligned}
$$

can be computed as

$$
\begin{aligned}
& \sum_{i=1}^{a+b} S q^{i}\left(\sum_{j+k=i} \overline{x_{2 a-j}(\xi) \otimes v_{b-k} v_{b} U_{\eta}}\right) \\
& \in H^{2+2 b}\left(\left(T \xi / T \xi\left\langle v_{a}\right\rangle\right) \wedge T \eta \bigcup_{\rho \alpha \wedge \beta} e^{2 a+2 b}, Z / 2\right) .
\end{aligned}
$$

This follows easily from the existence of the commutative diagram

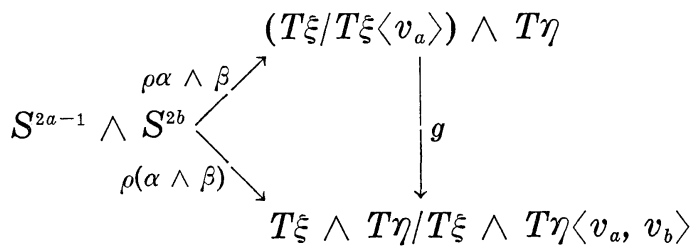


and computation of the cohomology maps in Diagram 10.11.

Step 4. Finally, we employ the commutative diagram

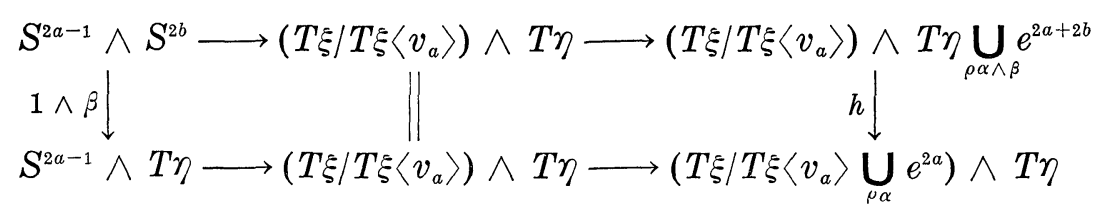

to show that

$$
\begin{aligned}
& \sum_{i=1}^{a+b} S q^{i} \sum_{j+k=i} \overline{x_{2 a-j}(\xi) \otimes v_{b-k} v_{b} U_{\eta}} \\
& =h^{*} \sum_{i=1}^{a+b} S q^{i} \sum_{j+k=1} \bar{x}_{2 a-j}(\xi) \otimes v_{b-k} v_{b} U_{\eta} \\
& =h^{*} \sum_{j=1}^{a} S q^{j}\left(\bar{x}_{2 \alpha-j}(\xi)\right) \otimes v_{b}^{2} U_{\eta} \\
& =\phi_{\xi}(\alpha) \cdot\left\langle\beta^{*} \Phi\left(V^{2}\right),\left[S^{2 b}\right]\right\rangle \in Z / 2 .
\end{aligned}
$$

This completes our outline of the proof of 10.8. We leave the details of Steps 2,3, and 4 to the reader.

We point out here that, if $\xi \rightarrow B \xi$ is a spherical fibration, $\pi_{1}(B \xi)=0$, then we have proved

THEOREM 10.12. The following conditions are equivalent:

(a) Every map $f: M^{q+2 n-1} \rightarrow T \xi^{q}, q \gg 2 n-1, M$ an unoriented manifold, is cobordant to a globally transversal map $g: N^{q+2 n-1} \rightarrow T \xi^{q}$.

(b) $\tilde{\mathscr{K}}(\xi) \equiv 0$.

(c) $\dot{\phi}: \pi_{2^{*}-1}(T \xi \wedge M O) \rightarrow Z / 2$ vanishes.

(Note that (c) is equivalent to the assertion that

$$
\pi_{2 n-1}\left((T \xi \wedge M O)\left\langle v_{n}\right\rangle\right) \longrightarrow \pi_{2 n-1}(T \xi \wedge M O)
$$

is surjective, $n \geqq 1$. We can replace $M P L$ by $M O$ because the restricted homomorphism $\widetilde{s}: \widetilde{\eta}_{2^{*}-1}(T \xi) \rightarrow Z / 2$ is sufficient to define $\tilde{\mathscr{K}}(\xi) \in H^{2^{*-1}}(B \xi, Z / 2)$.)

Finally, we construct the map $\alpha: K^{q+4} \rightarrow M S G_{q}$ used in 8.2. Recall $K^{q+4}=S^{q+3} \times I /(x, 0) \equiv(-x, 1)$ is a $Z / 2$-manifold with $\delta K^{q+4}=S^{q+3}$. Since $\pi_{3}(M S G)=Z / 2$, there is a map $\alpha: K^{q+4} \rightarrow M S G_{q}$ such that $\left.\alpha\right|_{\delta K^{4}}$ : $S^{q+3} \rightarrow M S G_{q}$ is the generator of $\pi_{3}(M S G)$. Therefore, $s \delta\left(K^{q+4}, \alpha\right)=$ $\hat{s}\left(S^{q+3},\left.\alpha\right|_{S^{q+3}}\right) \neq 0$. This establishes 8.2(i). Now there exist maps $\beta: S^{q+4} \rightarrow M S G_{q}$ with $\left\langle\beta^{*} \Phi\left(v_{2}^{2}\right),\left[S^{q+4}\right]\right\rangle \neq 0$. (For example, $\beta=p([C P(2)]$ ), $\left.p: \Omega_{*}(p t) \rightarrow \pi_{*}(M S G)\right)$. Thus, if necessary, we can change $\alpha: K^{q+4} \rightarrow$ $M S G_{q}$ on a disc $D^{q+4} \subset K^{q+4}$ such that $\left\langle\alpha^{*} \Phi\left(V^{2}\right),\left[K^{q+4}\right]\right\rangle=0$. This establishes 8.2(ii). 
11. Homotopy definition of $s_{n}: \Omega_{4^{*}+1}(T \xi, Z / n) \rightarrow Z / n$. Our next goal is a homotopy-theoretic definition of $s_{n}: \widetilde{\Omega}_{4^{*}+1}^{P L}(T \xi, Z / n) \rightarrow Z / n, \xi$ oriented. By Lemma 3.7, we need only consider $s_{2} r, r \geqq 1$. Moreover, given $f: M^{q+4 n+1} \rightarrow T \xi^{q}$, we can assume $\left.f\right|_{\delta M}$ is globally transversal, and then $s_{2} r\left(\left[M^{q+4 n+1}, f\right]\right)$ is computed as index $\left(L^{4 n}\right) \in Z / 8$ (interpreted as an element of $\left.Z / 2^{r}\right)$, where $L^{4 n}=f^{-1}\left(B \xi^{q}\right) \cap \delta M$ is a closed, $4 n$ dimensional $\mathrm{PD}$ space.

We will define, for any oriented spherical fibration $\eta$, a homomorphism $i_{\eta}: \pi_{4 n}(T \eta) \rightarrow Z / 8$ with the following property: If $\alpha: S^{q+4 n} \rightarrow T \eta^{q}$ is globally Poincaré-transversal, with $\alpha^{-1}\left(B \eta^{q}\right)=L^{4 n} \subset S^{q+4 n}$, then $i_{\eta}(\alpha)=\operatorname{index}\left(L^{4 n}\right)(\bmod 8) . \quad$ As in the beginning of $\S 10$, there is a natural Pontrjagin-Thom isomorphism $p: \widetilde{\Omega}_{*}^{P L}(T \xi) \stackrel{\sim}{\rightarrow} \pi_{*}(T \xi \wedge M S P L)$. Moreover, if $f: N \rightarrow T \xi$ is globally transversal, then $p([N, f]): S \rightarrow$ $T \xi \wedge M S P L$ is globally transversal, with $(p([N, f]))^{-1}(B \xi \times B S P L)=$ $f^{-1}(B \xi)$ as P.D. spaces. It follows that $s_{2^{r}}: \widetilde{\Omega}_{4^{*}+1}^{P L}\left(T \xi, Z / 2^{r}\right) \rightarrow Z / 2^{r}$ is equal to the composition

$$
\widetilde{\Omega}_{4^{*}+1}^{P L}\left(T \xi, Z / 2^{r}\right) \stackrel{\delta}{\longrightarrow} \widetilde{\Omega}_{4^{*}}^{P L} \stackrel{p}{\longrightarrow} \pi_{4^{*}}(T \xi \wedge M S P L) \stackrel{i}{\longrightarrow} Z / 8,
$$

with image interpreted in $Z / 2^{r}$.

Thus we need to define $i: \pi_{4 n}(T \eta) \rightarrow Z / 8$. The key idea is this: If $L^{4 n}$ is a closed, oriented PD space, then the Wu class $v_{2 n}(L) \epsilon$ $H^{2 n}(L, Z / 2)$ is the reduction of an integral class $\hat{v} \in H^{2 n}(L, Z)$, and, for any such choice of $\hat{v},\left\langle\hat{v}^{2},\left[L^{4 n}\right]\right\rangle \equiv \operatorname{index}\left(L^{4 n}\right)(\bmod 8)$. Suppose the normal fibration of $L$ admits an $\eta$-orientation, $\nu: L \rightarrow B \eta$. Let $K(Z, 2 n) \rightarrow B \eta\left\langle\beta v_{2 n}\right\rangle \rightarrow B \eta$ be the fibration with $k$-invariant $\beta v_{2 n}(\eta) \in$ $H^{2 n+1}(B \eta, Z)$. Then $\nu$ lifts to $\widetilde{\nu}: L \rightarrow B \eta\left\langle\beta v_{2 n}\right\rangle$, and, on the Thom space level, $\alpha=p[L, \nu]: S^{4 n} \rightarrow T \nu_{L} \rightarrow T \eta$ lifts to $\tilde{\alpha}: S^{4 n} \rightarrow T \nu_{L} \rightarrow T \eta\left\langle\beta V_{2 n}\right\rangle$. Moreover, if $\hat{v} \in H^{2 n}\left(B \eta\left\langle\beta v_{2 n}\right\rangle, Z\right)$ satisfies

$$
\rho_{2}(\hat{v})=\pi^{*}\left(v_{2 n}(\eta)\right) \in H^{2 n}\left(B \eta\left\langle\beta v_{2 n}\right\rangle, Z / 2\right),
$$

then index $\left(L^{4 n}\right) \equiv\left\langle\widetilde{\alpha}^{*} \Phi\left(\hat{v}^{2}\right),\left[S^{4 n}\right]\right\rangle(\bmod 8)$.

Theorem 11.1. Given $\alpha: S^{4 n} \rightarrow T \eta$, there exist liftings $\tilde{\alpha}: S^{4 n} \rightarrow$ $T \eta\left\langle\beta v_{2 n}\right\rangle$. Moreover, $\left\langle\widetilde{\alpha}^{*} \Phi\left(\hat{v}^{2}\right),\left[S^{4 n}\right]\right\rangle \in Z / 8$ is independent of the choice of the class $\hat{v}$ and the lifting $\tilde{\alpha}$.

We therefore $i: \pi_{4 n}(T \eta) \rightarrow Z / 8$ by $i(\alpha)=\left\langle\tilde{\alpha}^{*} \Phi\left(v^{2}\right),\left[S^{4 n}\right]\right\rangle \in Z / 8$. The point of Theorem 11.1 is that $i(\alpha) \in Z / 8$ is now defined for all $\alpha: S^{4 n} \rightarrow$ $T \eta$, regardless of whether $\alpha$ is transversal. If $\alpha: S^{4 n} \rightarrow T \eta$ is transversal, $i(\alpha)$ gives the index $(\bmod 8)$ of the inverse image of $B \eta$ because of the discussion above.

Proof of 11.1. Let $z \in H^{2 n+1}\left(B \eta, B \eta\left\langle\beta v_{2 n}\right\rangle, Z\right)=Z$ be a generator. 
The map on base spaces

$$
B \eta, B \eta\left\langle\beta v_{2 n}\right\rangle \stackrel{\Delta}{\longrightarrow} B \eta \times\left(B \eta, B \eta\left\langle\beta v_{2 n}\right\rangle\right) \stackrel{1 \times z}{\longrightarrow} B \eta \times(K(Z, 2 n+1), e)
$$

induces a map on Thom spaces

$$
T \eta / T \eta\left\langle\beta v_{2 n}\right\rangle \stackrel{g_{\eta}}{\longrightarrow} T \eta \wedge K(Z, 2 n+1) .
$$

One can prove the following, just as Theorem 10.1 is proved in [1].

LEMMA 11.2 .

$$
\text { (i ) } g_{\eta}^{*}: H^{i}(T \eta \wedge K(Z, 2 n+1), Z) \longrightarrow H^{i}\left(T \eta / T \eta\left\langle\beta v_{2 n}\right\rangle, Z\right)
$$

is an isomorphism if $i<4 n+2$, and is a surjection with kernel = $Z / 2$ if $i=4 n+2$. The generator of kernel $\left(g_{\eta}^{*}\right)$ in dimension $4 n+2$ is the element

$$
\begin{aligned}
& \beta v_{2 n} U \otimes c_{2 n+1}+U \otimes c_{2 n+1}^{2}=\beta\left(v_{2 n} U \otimes \iota_{2 n+1}+U \otimes S q^{2 n} \iota_{2 n+1}\right) \\
& =\beta\left(\sum_{i=1}^{n} S q^{2 i}\left(v_{2 n-2 i} U \otimes \iota_{2 n+1}\right)\right) \in H^{4 n+2}(T \eta \wedge K(Z, 2 n+1), Z) .
\end{aligned}
$$

(Recall that since $\eta$ is oriented, $v_{j}(\eta)=0$ if $j$ is odd.)

(ii) If $y \in H^{*}(B \eta, Z / p)$, p prime, then $\rho^{*} g_{\eta}^{*}(y U \otimes \iota)=\beta v_{2 n} \cdot y \cdot U \epsilon$ $H^{*}(T \eta, Z / p)$, where $\rho: T \eta \rightarrow T \eta / T \eta\left\langle\beta v_{2 n}\right\rangle$.

CoROLLARY 11.3. (i) $\left(g_{\eta}\right)_{*}: \pi_{i}\left(T \eta / T \eta\left\langle\beta v_{2 n}\right\rangle\right) \rightarrow \pi_{i}(T \eta \wedge K(Z, 2 n+1))$ is an isomorphism if $i \leqq 4 n$.

(ii) $\left(g_{\eta}\right)_{*} \rho_{*}: \pi_{\imath}(T \eta) \rightarrow \pi_{i}(T \eta \wedge K(Z, 2 n+1))$ is zero, $i \leqq 4 n$.

Proof. (i) is immediate from Lemma 11.2(i). To prove (ii), first note that $T \eta \wedge K(Z, 2 n+1)$ is the Eilenberg-MacLane spectrum $K\left(H_{*-2 n-1}(T \eta, Z)\right)$, through dimension $4 n$. It thus suffices to prove that, for all $y \in H^{j}(B \eta, Z / p), j<2 n, \rho^{*} g_{\eta}^{*}(y U \otimes \iota) \in H^{2 n+1+j}(T \eta, Z / p)$ is decomposable over the $\bmod p$ Steenrod algebra. (We assume here that $H_{*}(T \eta, Z)$ is of finite type.) If $p$ is odd, this is obvious from Lemma 11.2(ii). If $p=2$, we have

$$
\begin{aligned}
\rho^{*} g_{\eta}^{*} & (y U \otimes \iota)=\left(S q^{1} v_{2 n}\right) \cdot y \cdot U=S q^{1}\left(v_{2 n} \cdot y \cdot U\right)+v_{2 n} \cdot\left(S q^{1} y\right) \cdot U \\
& =S q^{1}\left(v_{2 n} \cdot y \cdot U\right)+\sum_{i=1}^{n} S q^{2 i}\left(v_{2 n-2 i} \cdot\left(S q^{1} v\right) \cdot U\right)+S q^{2 n}\left(S q^{1} y\right) \cdot U \\
& =S q^{1}\left(v_{2 n} \cdot y \cdot U\right)+\sum_{i=1}^{n} S q^{2 i}\left(v_{2 n-2 i}\left(S q^{1} y\right) \cdot U\right)+S q^{1}\left(y \cdot\left(S q^{1} y\right) \cdot U\right)
\end{aligned}
$$

From Corollary 11.3(i) and (ii), we see that

$$
\rho_{*}: \pi_{4 n}(T \eta) \longrightarrow \pi_{4 n}\left(T \eta / T \eta\left\langle\beta v_{2 n}\right\rangle\right)
$$


is zero, and the first statement of Theorem 11.1 follows.

We must now prove that, given $\alpha \in \pi_{4 n}(T \eta),\left\langle\tilde{\alpha}^{*} \Phi\left(\hat{v}^{2}\right),\left[S^{4 n}\right]\right\rangle \in Z / 8$ is independent of $\hat{v} \in H^{2 n}\left(B \eta\left\langle\beta v_{2 n}\right\rangle, Z\right)$ lifting

$$
\pi^{*}\left(v_{2 n}(\eta)\right) \in H^{2 n}\left(B \eta\left\langle\beta v_{2 n}\right\rangle, Z / 2\right),
$$

and independent of $\tilde{\alpha}: S^{4 n} \rightarrow T \eta\left\langle\beta v_{2 n}\right\rangle$ lifting $\alpha: S^{4 n} \rightarrow T \eta$. First, $\hat{v}$ may be replaced by $\hat{v}+2 y, y \in H^{2 n}\left(B \eta\left\langle\beta v_{2 n}\right\rangle, Z\right)$. Then $(\hat{v}+2 y)^{2}=$ $\hat{v}^{2}+4 \hat{v} y+4 y^{2}$. It thus suffices to prove that $\Phi\left(\rho_{2}\left(\hat{v} y+y^{2}\right)\right)=v_{2 n} \cdot y U+$ $y^{2} U$ is decomposable over the Steenrod algebra. But

$$
\begin{aligned}
v_{2 n} \cdot y U+y^{2} U & =y \cdot \chi\left(S q^{2 n}\right) U+S q^{2 n}(y) \cdot U \\
& =\sum_{i=1}^{n} S q^{2 i}\left(V_{2 n-2 i} \cdot y \cdot U\right) .
\end{aligned}
$$

Secondly, $\tilde{\alpha} \in \pi_{4 n}\left(T \eta\left\langle\beta v_{2 n}\right\rangle\right)$ may be replaced by $\tilde{\alpha}+\partial \beta, \beta \in$ $\pi_{4 n+1}\left(T \eta / T \eta\left\langle\beta v_{2 n}\right\rangle\right)$. The result that $i(\alpha)=\left\langle\widetilde{\alpha}^{*} \Phi\left(\hat{v}^{2}\right),\left[S^{4 n}\right]\right\rangle \in Z / 8$ is independent of $\widetilde{\alpha}$ follows immediately from

LEMMA 11.4. Given any

$$
\beta: S^{4 n+1} \longrightarrow T \eta \mid T \eta\left\langle\beta v_{2 n}\right\rangle,\left\langle\beta^{*} \delta \Phi\left(\hat{v}^{2}\right),\left[S^{4 n+1}\right]\right\rangle \equiv 0(\bmod 8),
$$

where $\delta: H^{4 n}(T \eta, Z) \rightarrow H^{4 n+1}\left(T \eta / T \eta\left\langle\beta v_{2 n}\right\rangle, Z\right)$.

Proof. We assert that $\delta \Phi\left(\hat{v}^{2}\right)=4 y$, where $\rho_{2}(y)$ is decomposable over the Steenrod algebra. Specifically,

$$
\begin{aligned}
\rho_{2}(y) & =g_{\eta}^{*}\left(v_{2 n} U \otimes \iota+U \otimes S q^{2 n} \iota\right) \\
& =\sum_{i=1}^{n} S q^{2 i}\left(g_{\eta}^{*}\left(v_{2 n-2 i} U \otimes \iota\right)\right) \in H^{4 n+1}\left(T \eta / T \eta\left\langle\beta v_{2 n}\right\rangle, Z / 2\right),
\end{aligned}
$$

where $g_{\eta}: T \eta / T \eta\left\langle\beta v_{2 n}\right\rangle \rightarrow T \eta \wedge K(Z / 2 n+1)$ was defined above. The lemma is immediate, given this assertion.

To see that $\delta \Phi\left(\hat{v}^{2}\right) \equiv 0(\bmod 4)$ is easy. If $\pi: \beta \eta\left\langle\beta v_{2 n}\right\rangle \rightarrow B \eta$, we have $\pi^{*}\left(v_{2 n}\right)=\rho_{2}(\hat{v})$, hence $\pi^{*}\left(\mathscr{P}\left(v_{2 n}\right)\right)=\rho_{4}\left(\hat{v}^{2}\right)$, where

$$
\mathscr{P}\left(v_{2 n}\right) \in H^{4 n}(B \eta, Z / 4)
$$

is the Pontrjagin square of $v_{2 n}$. Thus $\rho_{4} \delta\left(\hat{v}^{2}\right)=0 \in H^{4 n+1}\left(B \eta, B \eta\left\langle\beta v_{2 n}\right\rangle\right.$, $Z / 4)$. We need more delicate information about

$$
\left(\delta \hat{v}^{2}\right) \in H^{4 n+1}\left(B \eta, B \eta\left\langle\beta v_{2 n}\right\rangle, Z\right),
$$

so we work at the cochain level. Let $c \in C^{2 n}(B \eta, Z)$ be a cochain such that $\pi^{\sharp}(c) \in Z^{2 n}\left(B \eta\left\langle\beta v_{2 n}\right\rangle, Z\right)$ represents $\hat{v} \in H^{2 n}\left(B \eta\left\langle\beta v_{2 n}\right\rangle, Z\right)$ and such that $\rho_{2}(c) \in Z^{2 n}(B \eta B \eta, Z / 2)$ represents $v_{2 n} \in H^{2 n}(B \eta, Z / 2)$. Consider the cochain $c \cup c+\delta c \bigcup_{1} c \in C^{4 n}(B \eta, Z)$. (This is a $Z / 4$-cocycle which re- 
presents $\mathscr{P}\left(v_{2 n}\right) \in H^{4 n}(B \eta, Z / 4)$.) Since

$$
\pi^{\sharp}(\delta c)=\delta\left(\pi^{\sharp} c\right)=0, \pi\left(c \cup c+\delta c \bigcup_{1} c\right) \in Z^{4 n}\left(B \eta\left\langle\beta v_{2 n}\right\rangle, Z\right)
$$

represents $\hat{v}^{2}$. Thus $\delta\left(\hat{v}^{2}\right) \in H^{4 n+1}\left(B \eta, B \eta\left\langle\beta v_{2 n}\right\rangle, Z\right)$ is represented by the relative cocycle $\delta\left(c \cup c+\delta c \bigcup_{1} c\right)=2(\delta c) \cup c+\delta c \bigcup_{1} \delta c$ (see [23], Theorem 5.1 for the coboundary of $\bigcup_{1}$-products). On the other hand, $\delta c=2 d$, where $d \in Z^{2 n+1}\left(B \eta, B \eta\left\langle\beta v_{2 n}\right\rangle, Z\right)$ represents the generator $z \in H^{2 n+1}\left(B \eta, B \eta\left\langle\beta v_{2 n}\right\rangle, Z\right)$. Thus $\delta\left(\hat{v}^{2}\right)$ is represented by the relative cocycle $4\left(d \cup c+d \cup_{1} d\right)$. Clearly, $\rho_{2}\left(d \cup c+d \cup_{1} d\right)$ represents the cohomology class $v_{2 n} \cdot z+S q^{2 n}(z) \in H^{4 n+1}\left(B \eta, B \eta\left\langle\beta v_{2 n}\right\rangle, Z / 2\right)$.

Now consider the maps

$$
B \eta, B \eta\left\langle\beta v_{2 n}\right\rangle \stackrel{\Delta}{\longrightarrow} B \eta \times\left(B \eta, B \eta\left\langle\beta v_{2 n}\right\rangle\right) \stackrel{1 \times z}{\longrightarrow} B \eta \times(K(Z, 2 n+1), e),
$$

which induce on the level of Thom spaces the map

$$
g_{\eta}: T \eta / T \eta\left\langle\beta v_{2 n}\right\rangle \longrightarrow T \eta \wedge K(Z, 2 n+1) \text {. }
$$

Under the "Thom isomorphism"

$$
H^{*}\left(B \eta, B \eta\left\langle\beta v_{2 n}\right\rangle, Z / 2\right) \stackrel{\sim}{\longrightarrow} H^{*}\left(T \eta / T \eta\left\langle\beta v_{2 n}\right\rangle, Z / 2\right),
$$

$v_{2 n} \cdot z$ corresponds to $g_{\eta}^{*}\left(v_{2 n} U \otimes \iota_{2 n+1}\right)$, and $S q^{2 n}(z)$ corresponds to $g_{r}^{*}\left(U \otimes S q^{2 n} c_{2 n+1}\right)$. Thus $\delta \Phi\left(\hat{v}^{2}\right)=4 y$, where

$$
\rho_{2}(y)=g_{\eta}^{*}\left(v_{2 n} U \otimes \iota+U \otimes S q^{2 n} \iota\right),
$$

as asserted.

Finally, we point out that, if $\xi \rightarrow B \xi$ is an oriented spherical fibration, $\pi_{1}(B \xi)=0$ and if $\tilde{\mathscr{L}}(\xi) \in H^{4^{*+1}}\left(B \xi, Z_{(2)}\right)$ is the class defined in $\S 5$ (and in this section), then we have proved

THEOREM 11.5. The following conditions are equivalent:

(a) Every map $f: M^{q+4 n+1} \rightarrow T \xi^{q}, q \gg 4 n+1, M a Z / 2^{r}$-manifold, is cobordant to a globally transversal map $g: N^{q+4 n+1} \rightarrow T \xi^{q}$.

(b) $\tilde{\mathscr{L}}(\xi) \equiv 0$.

(c) $i: \pi_{4^{*}}(T \xi \wedge M S O) \rightarrow Z / 8$ vanishes on the torsion subgroup of $\pi_{4^{*}}(T \xi \wedge M S O)$.

(Note that (c) is equivalent to the assertion that

$$
i \delta: \pi_{4^{*}+1}\left(T \xi \wedge M S O, Z / 2^{2}\right) \longrightarrow \pi_{4^{*}}(T \xi \wedge M S O) \longrightarrow Z / 8
$$

vanishes. We can replace $M S P L$ by $M S O$ since the restricted homomorphisms $s_{2^{r}}: \widetilde{\Omega}_{4^{*}+1}\left(T \xi, Z / 2^{r}\right) \rightarrow Z / 2^{r}, r \geqq 1$, are sufficient to define the class $\tilde{\mathscr{L}}(\xi) \in H^{4^{*+1}}\left(B \xi, Z_{(2)}\right)$.) 
APPENDIX. Constructing cohomology classes from homomorphisms on bordism. In this Appendix, we review the technique used by Sullivan (and earlier by Thom in his definition of rational $P L$ Pontrjagin classes) of defining cohomology classes of a space $Y$ in terms of homomorphisms on the smooth bordism of $X$. We deal separately with cohomology classes with $Z / 2-, Q-, Z_{(2)}$, and $Z / 2^{r-}$ coefficients.

Case $Z / 2$. The key fact for the construction is the result of Thom, that the Hurewicz homomorphism $h_{n}: \mathfrak{R}_{n}(X) \rightarrow H_{n}(X, Z / 2)$ is surjective, and that kernel $\left(h_{n}\right)$ is generated over $Z / 2$ by the decomposable elements in the image of the natural pairing $\Re_{*}(X) \otimes \Re_{*}(p t) \rightarrow$ $\mathfrak{R}_{*}(X)$, that is, by elements $\left[M^{n-\imath}, f\right] \cdot\left[N^{i}\right]=\left[M \times N, f \pi_{1}\right]$, where $f$ : $M^{n-\imath} \rightarrow X$ and $i>0$.

As an immediate consequence, we have that a cohomology class $z_{n} \in H^{n}(X, Z / 2)$, which is the same as a homomorphism $H_{n}(X, Z / 2) \rightarrow$ $Z / 2$, is equivalent to a homomorphism $\phi_{n}: \mathfrak{R}_{n}(X) \rightarrow Z / 2$ such that $\phi([M, f] \cdot[N])=0$ whenever $\operatorname{dim}(N)>0$.

In practice, the homomorphisms $\phi_{*}: \mathfrak{R}_{*}(X) \rightarrow Z / 2$ which arise do not vanish on decomposables, but satisfy a product formula like

$$
\dot{\phi}_{*}([M, f][N])=\phi_{*}([M, f]) \cdot \chi(N),
$$

where $\chi(N)$ is the Euler characteristic of $N$, modulo 2. In such a situation, we obtain a (graded) cohomology class $z_{*}=z_{0}+z_{1}+z_{2}+\cdots$, $z_{\imath} \in H^{i}(X, Z / 2)$, as follows. Perturb the Hurewicz homomorphism $h$ to $\widetilde{h}: \mathfrak{N}_{*}(X) \rightarrow H_{*}(X, Z / 2)$ by setting

$$
\widetilde{h}(M, f)=f_{*}\left(V^{2}(M) \cap[M]\right) \in H_{*}(X, Z / 2),
$$

where $V^{2}(M)=1+v_{1}^{2}(M)+v_{2}^{2}(M)+\cdots$ is the square of the total Wu class of $M$. The homomorphism $\widetilde{h}$ is not homogeneous; that is, $\widetilde{h}$ is not degree-preserving. However, $\widetilde{h}$ is still surjective, and kernel $(\widetilde{h})$ is generated over $Z / 2$ by elements $[M, f] \cdot[N]-\left[M, f\left[\left\langle V^{2}(N),[N]\right\rangle\right.\right.$, where $\left\langle V^{2}(N),[N]\right\rangle=\chi(N) \in Z / 2$. Thus we have

Proposition A.1. Graded classes $z_{*} \in H^{*}(X, Z / 2)$ correspond bijectively to homomorphisms $\dot{\phi}_{*}: \mathfrak{R}_{*}(X) \rightarrow Z / 2$ which satisfy $\dot{\phi}_{*}([M, f][N])=$ $\dot{\phi}_{*}([M, f]) \cdot \chi(N) \in Z / 2$. The correspondence is defined by the equation

$$
\phi_{*}(M, f)=\left\langle V^{2}(M) \cdot f^{*}\left(z_{*}\right),[M]\right\rangle \in Z / 2 .
$$

Proof. This follows easily from the above properties of $\tilde{h}: \mathfrak{P}_{*}(X) \rightarrow$ $H_{*}(X, Z / 2)$, the relation $V^{2}(M \times N)=V^{2}(M) \otimes V^{2}(N) \in H^{*}(M, Z / 2) \otimes$ $H^{*}(N, Z / 2)=H^{*}(M \times N, Z / 2)$, and the computation 


$$
\left\langle V^{2}(M) \cdot f^{*}\left(z_{*}\right),[M]=\left\langle f^{*}\left(z_{*}\right), V^{2}(M) \cap[M]\right\rangle=\left\langle z_{*}, \tilde{h}[M, f]\right\rangle .\right.
$$

REMARK A.2. Since $V^{2}=1+v_{1}^{2}+v_{2}^{2}+\cdots$ is nonzero only in even dimensions, we have that, if $f: M^{n} \rightarrow X$ is a map, then

$$
\phi_{n}\left(M^{n}, f\right)=\left\langle V^{2}(M) \cdot f^{*}\left(\sum_{\imath \geq 0} z_{n-2 i}\right),[M]\right\rangle .
$$

That is, the homomorphism $\phi_{n}, n$ even, is determined by the classes $z_{0}, z_{2}, z_{4}, \cdots$, and the homomorphism $\phi_{n}, n$ odd, is determined by the classes $z_{1}, z_{3}, z_{5}, \cdots$.

$Z / 2$-cohomology classes can also be defined by using the homology theory "bordism with $Z / 2$-coefficients," $\Omega_{*}(, Z / 2)$. Namely, $\Omega_{*}(X, Z / 2)$ is a module over $\Omega_{*}(p t, Z / 2)$, the Hurewicz homomorphism

$$
h: \Omega_{*}(X, Z / 2) \longrightarrow H_{*}(X, Z / 2)
$$

is surjective, and kernel $(h)$ is generated by decomposable elements $[M, f] \cdot[N]$. As in Proposition A.1, we deduce

Proposition A.3. A graded class $z_{*} H^{*}(X, Z / 2)$ is equivalent to a homomorphism $\phi_{*}: \Omega_{*}(X, Z / 2) \rightarrow Z / 2$ which satisfies $\phi_{*}([M, f] \cdot[N])=$ $\phi_{*}([M, f]) \cdot \chi(N)$. The correspondence is defined by

$$
\phi_{*}([M, f])=\left\langle Y^{2}(M) \cdot f^{*}\left(z_{*}\right),[M]\right\rangle \in Z / 2 .
$$

REMARK A.4. Since for $Z / 2$-manifolds $M, w_{1}^{2}(M)=0$, and since $v_{2 i+1}$ is always divisible by $w_{1}$, we have that $V^{2}(M)=1+v_{2}^{2}(M)+$ $v_{4}^{2}(M)+\cdots$ is nonzero only in dimensions divisible by 4 . Thus

$$
\dot{\phi}_{n}\left(M^{n}, f\right)=\left\langle V^{2}(M) \cdot f^{*}\left(\sum_{\imath \geq 0} z_{n-4 i}\right),\left[M^{n}\right]\right\rangle \in Z / 2 .
$$

Case $Q$. Another simple case when cohomology classes are directly constructed from homomorphisms on bordism is the case of rational coefficients. Specifically, the Hurewicz homomorphism $h: \Omega_{*}(X) \otimes Q \rightarrow$ $H_{*}(X, Q)$ is again surjective and kernel $(h)$ is generated, over $Q$, by decomposable elements $[M, f] \cdot[N],] M, f] \in \Omega_{*}(X),[N] \in \Omega_{*}(p t)$, and $\operatorname{dim}(N)>0$. Thus homomorphisms $\phi_{*}: \Omega_{*}(X) \otimes Q \rightarrow Q$ which vanish on decomposables yield rational cohomology classes.

In practice, the homomorphisms $\phi_{*}: \Omega_{*}(X) \otimes Q \rightarrow Q$ which arise satisfy a product formula like $\phi([M, f] \cdot[N])=\phi_{*}([M, f] \cdot \operatorname{index}(N)$. Let $L_{Q}(M)=1+L_{1}(M)+L_{2}(M)+\cdots \in H^{4^{*}}(M, Q)$ be the (inverse) Hirzebruch polynomial of the normal bundle of $M$ (so that $\left\langle L_{Q}(M),[M]=\right.$ index $(M) \in Z)$. Then we have

Proposition A.5. Graded classes $z_{*} \in H^{*}(X, Q)$ correspond bijec- 
tively with homomorphisms $\phi_{*}: \Omega_{*}(X) \otimes Q \rightarrow Q$ which satisfy $\phi_{*}([M, f] \cdot[N])=\phi_{*}([M, f]) \cdot$ index $(N)$. The correspondence is defined by the equation

$$
\phi_{n}\left(M^{n}, f\right)=\left\langle L_{Q}\left(M^{n}\right) \cdot f^{*}\left(\sum_{i \geq 0} z_{n-4 i}\right),\left[M^{n}\right]\right\rangle \in Q .
$$

Case $Z_{(2)}$. A somewhat more complicated situation arises if we attempt to define a cohomology class with $Z_{(2)}$-coefficients. A complete discussion is given in [15]. We state here only the final results of that discussion. Roughly, the idea is that a $Z_{(2)}$-class determines by obvious coefficient maps (and is determined by !) a $Q$-class and a compatible set of $Z / 2^{r}$-classes, $r \geqq 1$.

Here are the relevant facts.

(1) The evaluation map $H^{n}\left(X, Z / 2^{r}\right) \stackrel{\sim}{\rightarrow} \operatorname{Hom}\left(H_{n}\left(X, Z / 2^{r}\right), Z / 2^{r}\right)$ is an isomorphism.

(2) The Hurewicz map $h_{*}\left(2^{r}\right): \Omega_{*}\left(X, Z / 2^{r}\right) \rightarrow H_{*}\left(X, Z / 2^{r}\right)$ is surjective.

(3) There is a natural module structure

$$
\Omega_{*}\left(X, Z / 2^{r}\right) \otimes \Omega_{*}\left(p t, Z / 2^{r}\right) \longrightarrow \Omega_{*}\left(X, Z / 2^{r}\right)
$$

and, in the limit, kernel $\left(h_{*}\left(2^{\infty}\right): \Omega_{*}\left(X, Z / 2^{\infty}\right) \rightarrow H_{*}\left(X, Z / 2^{\infty}\right)\right)$ is generated by "products" $i([M, f] \cdot[N])$, where $Z / 2^{\infty}=\lim _{\longrightarrow} Z / 2^{r}, \quad i$ : $Z / 2^{r} \rightarrow Z / 2^{\infty}$.

(4) The rational $L$-class $L_{Q}=1+L_{1}+L_{2}+\cdots \in H^{4^{*}}(B S O, Q)$, used in Case $Q$ above, can be refined to a unique class $L \in H^{4 *}\left(B S O, Z_{(2)}\right)$ which satisfies $\rho_{Q}(L)=L_{Q} \in H^{4^{*}}(B S O, Q)$ and $\rho_{2}(L)=V^{2} \in H^{4^{*}}(B S O, Z / 2)$. Moreover, $L$ is multiplicative; that is, on Whitney sums of bundles, $L(\xi+\eta)=L(\xi) \cdot L(\eta)$

Using these facts, the following is proved in [15].

Proposition A.6. Graded classes $z_{*} \in H^{*}\left(X, Z_{(2)}\right)$ correspond bijectively with commutative diagrams

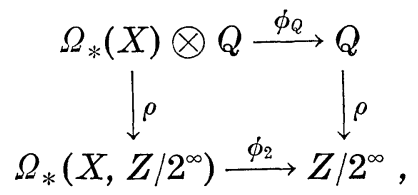

where $\rho: Q \rightarrow Q / Z_{(2)}=Z / 2^{\infty}$ is the projection, which satisfy

(i ) $\phi_{Q}([M, f] \cdot[N])=\phi_{Q}([M, f]) \cdot \operatorname{index}(N) \in Q$,

(ii) $\phi_{2}(i([P, g] \cdot[Q]))=\phi_{2}(i([P, g])) \cdot \operatorname{index}(Q) \in Z / 2^{r} \subset Z / 2^{\infty}$, 
where $P$ and $Q$ are $Z / 2^{r}$-manifolds, $i: Z / 2^{r} \rightarrow Z / 2^{\infty}$. The correspondence is defined by the equations

$$
\phi_{Q}\left(\left[M^{n}, f\right]\right)=\left\langle L\left(M^{n}\right) \cdot f^{*}\left(\sum_{i \geq 0} z_{n-4 i}\right),[M] \in Z_{(2)} \subset Q,\right.
$$

where $[M, f] \in \Omega_{n}(X)$, and

$$
\phi_{2}\left(i\left[P^{n}, g\right]\right)=\left\langle L\left(P^{n}\right) \cdot g^{*}\left(\sum_{i \geq 0} z_{n-4 i}\right),[P]\right\rangle \in Z / 2^{r} \subset Z / 2^{\infty},
$$

where $\left[P^{n}, g\right] \in \Omega_{n}\left(X, Z / 2^{r}\right)$.

REMARK A.7. It is clear that the torsion classes in $H^{*}\left(X, Z_{(2)}\right)$ correspond to the diagrams in A.6 with $\phi_{Q} \equiv 0$.

Case $Z / 2^{r}$. The final result that we need for this paper is a construction of cohomology classes with $Z / 2^{r}$-coefficients, $r>1$ (see $\S 8)$. Now, we have the isomorphism

$$
H^{*}\left(X, Z / 2^{r}\right) \stackrel{\sim}{\longrightarrow} \operatorname{Hom}\left(H_{*}\left(X, Z / 2^{r}\right), Z / 2^{r}\right)
$$

and the surjection $h_{*}\left(2^{r}\right): \Omega_{*}\left(X, Z / 2^{r}\right) \rightarrow H_{*}\left(X, Z / 2^{r}\right)$, as mentioned in Case $Z_{(2)}$ above. However, kernel $\left(h_{*}\left(2^{r}\right)\right)$ is somewhat complicated, and before we can define $Z / 2^{r}$-cohomology classes, we must study this kernel.

First, consider $h_{*}: \Omega_{*}(X) \rightarrow H_{*}(X, Z)$, which is surjective modulo the class of finite groups of odd order. We know that, since $M S O$ localized at 2 is an Eilenberg-MacLane spectrum, there is an isomorphism $\Omega_{*}(X)=H_{*}\left(X, \Omega_{*}(p t)\right)$ (mod groups of odd order). Thus there is an exact sequence

$$
\begin{aligned}
0 \longrightarrow & H_{*}(X, Z) \otimes \Omega_{*}(p t) \longrightarrow \Omega_{*}(X) \\
& \rightarrow \operatorname{Tor}_{*-1}\left(H_{*}(X, Z), \Omega_{*}(p t)\right) \longrightarrow 0
\end{aligned}
$$

(mod groups of odd order). We choose a subgroup of $\Omega_{*}(X)$ isomorphic to $H_{*}(X, Z)$ under $h, H_{*}(X, Z) \subset \Omega_{*}(X)$. Then elements in image $\left(H_{*}(X, Z) \otimes \Omega_{*}(p t) \rightarrow \Omega_{*}(X)\right)$ are clearly constructed as products $[M, f] \cdot[N],[M, f] \in H_{*}(X, Z),[N] \in \Omega_{*}(p t)$. Now the torsion in $\Omega_{*}(p t)$ consists of elements of order 2 ([24]). Let $f: P^{m} \rightarrow X$ represent an element of order 2 in $H_{m}(X, Z)$, and let $Q^{n} \in \Omega_{n}(p t)$ have order 2 . We construct an element $[P, f] *[Q] \in \Omega_{m+n+1}(X)$, which hits the torsion product $[P, f] *[Q] \in \operatorname{Tor}\left(H_{*}(X, Z), \Omega_{*}(p t)\right)$, as follows. Let $\widetilde{F}: \widetilde{P} \rightarrow X$ satisfy $\partial \widetilde{P}=2 P,\left.\widetilde{F}\right|_{\partial \widetilde{P}}=2 f$, and let $\partial \widetilde{Q}=2 Q$. Form the map

$$
\widetilde{F} \circ \pi_{1} \cup f \circ \pi_{1}: \widetilde{P} \times Q \underset{2 P \times Q}{\bigcup} P \times \widetilde{Q} \longrightarrow X .
$$


This is our desired element of $\Omega_{m+n+1}(X)$. Note that this element belongs to the Massey product $\langle[P, f], 2,[Q]\rangle$. Of course, it is not well-defined, but depends on the choices $\widetilde{P}, \widetilde{F}$, and $\widetilde{Q}$. The indeterminacy is clearly the same as that of the Massey product $\langle[P, f], 2$, $[Q]\rangle$, namely, the products with $[P, f]$ or $[Q]$ as one factor:

$$
[P, f] \otimes \Omega_{n+1}(p t)+\Omega_{m+1}(X) \otimes[Q] \subset \Omega_{m+n+1}(X) .
$$

We point out that $\widetilde{P}$ and $\widetilde{Q}$ give $Z / 2$-manifolds in the obvious way, and the Massey product constructed above is the Bockstein of the product of the $Z / 2$-manifolds $\widetilde{P}$ and $\widetilde{Q}$. That is ([15]),

$$
\langle[P, f], 2,[Q]\rangle=\delta([\widetilde{P}, \widetilde{F}) \otimes[\widetilde{Q}]),
$$

where $\delta: \Omega_{*+1}(X, Z / 2) \rightarrow \Omega_{*}(X)$ is the Bockstein.

The description of generators of $\Omega_{*}(X)$ given above easily implies

LEMmA A.8. kernel $\left(h_{*}: \Omega_{*}(X) \rightarrow H_{*}(X, Z)\right)$ is generated (mod groups of odd order) by

(i) Products $[M, f] \cdot[N], \operatorname{dim}(N)>0$ and

(ii) Bocksteins of products $\delta([\widetilde{P}, \widetilde{F}] \cdot[\widetilde{Q}])$, where $\widetilde{P}$ and $\widetilde{Q}$ are $Z / 2-m a n i f o l d s$ and $\operatorname{dim}(\widetilde{Q})>0$.

Next, we consider the relation between $\Omega_{*}(X)$ and $\Omega_{*}\left(X, Z / 2^{r}\right)$. There is a commutative diagram
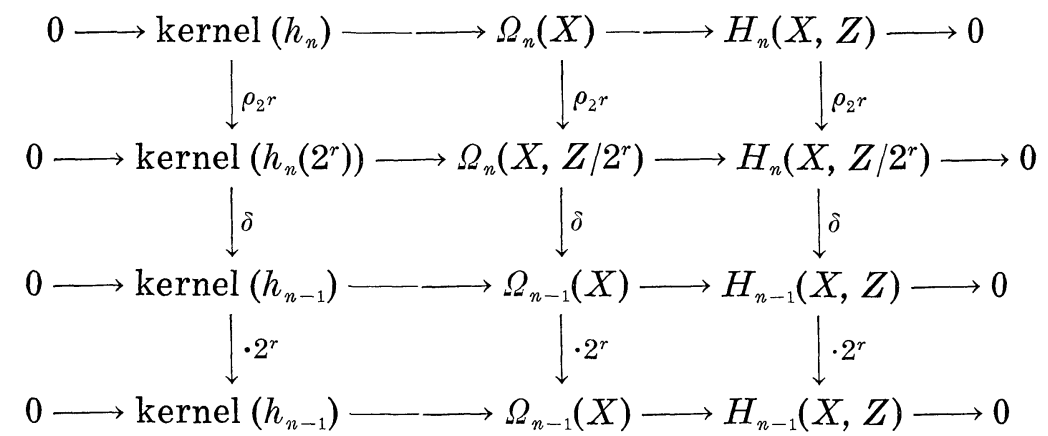

The columns are exact and the rows are exact modulo groups of odd order.

Lemma A.10. kernel $\left(h_{*}\left(2^{r}\right): \Omega_{*}\left(X, Z / 2^{r}\right) \rightarrow H_{*}\left(X, Z / 2^{r}\right)\right)$ is generated by

(i ) products $[M, f] \cdot[N],[M, f] \in \Omega_{*}\left(X, Z / 2^{r}\right),[N] \in \Omega_{*}(p t)$, and $\operatorname{dim}(N)>0$. 
(ii ) $Z / 2$-products $i([\tilde{M}, \tilde{f}] \cdot \tilde{N}],[\tilde{M}, \tilde{f}] \in \Omega_{*}(X, Z / 2),[\tilde{N}] \in \Omega_{*}(p t, Z / 2)$, $\operatorname{dim}(\tilde{N})>0$, and $i=2^{r-1}: \Omega_{*}(X, Z / 2) \rightarrow \Omega_{*}\left(X, Z / 2^{r}\right)$.

(iii) $Z / 2^{r}$-reductions of Bocksteins of products, $\rho_{2} \delta([\widetilde{P}, \widetilde{F}] \cdot[\widetilde{Q}])$, $[\widetilde{P}, \widetilde{F}] \in \Omega_{*}(X, Z / 2),[\widetilde{Q}] \in \Omega_{*}(p t, Z / 2)$, and $\operatorname{dim}(\widetilde{Q})>0$.

Proof. From Diagram A.9, we see that kernel $\left(h_{n}\left(2^{r}\right)\right)$ is generated by $\rho_{2^{r}}\left(\right.$ kernel $\left.\left(h_{n}\right)\right)$, together with a set of elements $\left\{x_{i}\right\}$ such that $\delta\left\{x_{\imath}\right\}=\operatorname{kernel}\left(h_{n-1}\right) \cap \operatorname{kernel}\left(2^{r}\right)$. By Lemma A.8, $\rho_{2^{r}}\left(\operatorname{kernel}\left(h_{n}\right)\right)$ is generated by $\rho_{2^{r}}([M, f] \cdot[N])=\left(\rho_{2^{r}}[M, f]\right) \cdot[N], \operatorname{dim}(M)+\operatorname{dim}(N)=$ $n$, and $\operatorname{dim}(N)>0$, together with $\rho_{2^{r}} \delta([\widetilde{P}, \widetilde{F}] \cdot[\widetilde{Q}]), \operatorname{dim}(\widetilde{P})+\operatorname{dim}(\widetilde{Q})=$ $n+1$, and $\operatorname{dim}(\widetilde{Q})>0$. These elements are of types (i) and (iii) respectively in A.10. Also, corresponding to the elements of the form $\delta([\widetilde{P}, \widetilde{F}] \cdot[\widetilde{Q}]) \in \operatorname{kernel}\left(h_{n-1}\right)$, where $\widetilde{P}$ and $\widetilde{Q}$ are $Z / 2$-manifolds, we include the elements $i([\widetilde{P}, \widetilde{F}] \cdot[\widetilde{Q}])$ in the set $\left\{x_{2}\right\} \subset \operatorname{kernel}\left(h_{n}\left(2^{r}\right)\right)$. These elements are of type (ii) in A.10.

Finally, suppose $[M, f] \cdot[N] \in \operatorname{kernel}\left(h_{n-1}\right) \cap\left(H_{*}(X, Z) \otimes \Omega_{*}(p t)\right)$, and $2^{r}[M, f] \cdot[N]=0$. We consider two cases: first, $2[N]=0 \in \Omega_{*}(p t)$ and, secondly, $2^{r}[M, f]=0 \in H_{*}(X, Z) \subset \Omega_{*}(X)$. It is easy to see these two cases suffice since $\Omega_{*}(p t)$ is a free group plus 2-torsion. In the first case, choose $\tilde{N}$ a $Z / 2$-manifold with $\delta \widetilde{N}=N$. Then $[M, f] \cdot[N]=\delta([M, f] \cdot[\tilde{N}])$, hence we can add the elements $i([M, f] \cdot[\tilde{N}])$ of type (ii) in A.10 to the set $\left\{x_{i}\right\}$. In the second case, let $[M, f]=$ $\delta[\tilde{M}, \widetilde{f}]$, where $\tilde{M}$ is a $Z / 2^{r}$-manifold. Then $\left.[M, f] \cdot[N]=\delta(\tilde{M}, \tilde{f}] \cdot[N]\right)$, hence we can add the elements $[\tilde{M}, \tilde{f}] \cdot[N]$ of type (i) in A.10 to the set $\left\{x_{i}\right\}$. This completes the proof, since now our set $\left\{x_{2}\right\}$ has the required property $\delta\left\{x_{i}\right\}=\operatorname{kernel}\left(h_{n-1}\right) \cap \operatorname{kernel}\left(2^{r}\right)$.

Proposition A.11. Graded $Z / 2^{r}$-cohomology classes $z_{*} \in H^{*}(X$, $\left.Z / 2^{r}\right)$ correspond bijectively with homomorphisms $\dot{\phi}_{*}: \Omega_{*}\left(X, Z / 2^{r}\right) \rightarrow$ $Z / 2^{r}$ which satisfy

( i ) $\phi_{*}([M, f][N])=\phi_{*}([M, f]) \cdot \operatorname{index}(N) \subset Z / 2^{r}$, where $[M, f] \epsilon$ $\Omega_{*}\left(X, Z / 2^{r}\right)$ and $[N] \in \Omega_{*}(p t)$,

(ii ) $\phi_{*}(i([\tilde{M}, \tilde{f}] \cdot[\tilde{N}]))=\phi^{*}(i[\tilde{M}, \widetilde{f}]) \cdot i n d e x(\tilde{N}) \in Z / 2 \subset Z / 2^{r}$, where $[\tilde{M}, \widetilde{f}] \in \Omega_{*}(X, Z / 2),[\widetilde{N}] \in \Omega^{*}(p t, Z / 2)$, and

(iii) $\phi_{*}\left(\rho_{2^{r}} \delta([\widetilde{P}, \widetilde{F}] \cdot[\widetilde{Q}])\right)=\phi_{*}\left(\rho_{2^{r}} \delta([\widetilde{P}, \widetilde{F}])\right) \cdot \operatorname{index}(\widetilde{Q}) \in Z / 2 \subset Z / 2^{r}$, where $[\widetilde{P}, \widetilde{F}] \in \Omega_{*}(X, Z / 2)$ and $[\widetilde{Q}] \in \Omega_{*}(p t, Z / 2)$. The defining equation is

$$
\dot{\phi}_{*}([M, f])=\left\langle\rho_{2^{r}} L(M) \cdot f^{*}\left(\sum_{\imath \geq 0} z_{n-4 \imath}\right),[M]\right\rangle \in Z / 2^{r}
$$

Proof. This follows easily from Lemma A.10, the discussion in the first paragraph of Case $Z / 2^{r}$, and properties of the class $L \in$ $H^{4^{*}}\left(B S O, Z_{(2)}\right)$. 
Remark A.12. If $r=1$, Proposition A.11 is simply Proposition A.3, since $\rho_{2} L=V^{2} \in H^{4^{*}}(B S O, Z / 2)$, and since, for $Z / 2$-manifolds, $\operatorname{index}(\widetilde{N})=\chi(\widetilde{N}) \in Z / 2$.

REMARK A.13. Given $\phi_{*}: \Omega_{*}\left(X, Z / 2^{r}\right) \rightarrow Z / 2^{r}$, consider the homomorphisms for $s \geqq 1$,

$$
\phi_{*} \rho_{2^{r}} \delta: \Omega_{n+1}\left(X, Z / 2^{s}\right) \longrightarrow \Omega_{n}(X) \longrightarrow \Omega_{n}\left(X, Z / 2^{r}\right) \longrightarrow Z / 2^{r} .
$$

If $s \leqq r$, image $\left(\phi_{*} \rho_{2^{r}} \delta\right) \subset Z / 2^{s} \subset Z / 2^{r}$. If $s \geqq r$, we compose $\phi_{n} \rho_{2^{r}} \delta$ with the inclusion $Z / 2^{r} \subset Z / 2^{s}$. It is easy to see that this gives a commutative diagram

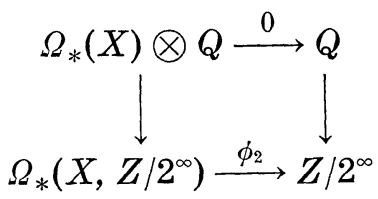

where $Z / 2^{\infty}=\underset{\rightarrow}{\lim } Z / 2^{s}$ and $\phi_{2}=\underset{\rightarrow}{\lim }\left(\left.\phi_{*} \rho_{2^{r}} \delta\right|_{\Omega_{*}\left(X, Z / 2^{s}\right)}\right)$. If $\phi_{*}: \Omega_{*}\left(X, Z / 2^{r}\right) \rightarrow$ $Z / 2^{r}$ satisfies the hypothesis of A.11 (hence gives classes $z_{n} \in H^{n}\left(X, Z / 2^{r}\right)$, $n \geqq 0$ ), then it is not hard to prove that the diagram A.14 above satisfies the hypothesis of A.6. The point is, the product formulae A.11(i) and (iii) imply the product formula A.6(ii). Thus diagram A.14 gives torsion $Z / 2$-classes $\mathscr{Z}_{n+1} \in H^{n+1}\left(X, Z_{(2)}\right)$. It is clear that $\mathscr{Z}_{n+1}=\beta z_{n}$ where $\beta$ is the Bockstein for the coefficient sequence

$$
0 \longrightarrow Z_{(2)} \stackrel{2^{r}}{\longrightarrow} Z_{(2)} \longrightarrow Z / 2^{r} \longrightarrow 0 \text {. }
$$

REMARK A.15. In the cases $Z / 2, Q$, and $Z_{(2)}$ above, all the theorems stated remain true if smooth bordism is replaced by $P L$-bordism. (In Case $Z_{(2)}$, one uses the result of [15] that $L \in H^{4^{*}}\left(B S O, Z_{(2)}\right)$ extends to a canonical class $L \in H^{4^{*}}\left(B S P L, Z_{(2)}\right)$.) The case $Z / 2^{r}$ is somewhat more complicated, since $\Omega_{*}^{P L}(p t)$ contains torsion of order $2^{s}$, all $s \geqq 1$. Thus kernel $\left(h_{*}^{P L}\left(2^{r}\right): \Omega_{*}^{P L}\left(X, Z / 2^{r}\right) \rightarrow H_{*}\left(X, Z / 2^{r}\right)\right)$ is complicated. However, one can show the following analog of Lemma A.10.

LeMma A.10(PL). kernel $\left(h_{*}^{P L}\left(2^{r}\right)\right) \subset \Omega_{*}^{P L}\left(X, Z / 2^{r}\right)$ is generated by

(i ) images of products $i([M, f] \cdot[N])$, where $[M, f] \in \Omega_{*}^{P L}\left(X, Z / 2^{s}\right)$, $[N] \in \Omega_{*}^{P L}\left(p t, Z / 2^{s}\right)$, and $\operatorname{dim}(N)>0, s \leqq r$, and $i: Z / 2^{s} \rightarrow Z / 2^{r}$ the inclusion.

(ii) $Z / 2^{r}$-reductions of Bocksteins of products, $\rho_{2^{r}} \delta([P, F] \cdot[Q])$, where $[P, F] \in \Omega_{*}^{P L}\left(X, Z / 2^{s}\right),[Q] \in \Omega_{*}^{P L}\left(p t, Z / 2^{s}\right)$, and $\operatorname{dim}(Q)>0$, and $s \leqq r$.

As a corollary, one obtains the following analog of Proposition 
Proposition A.11(PL). Graded $Z / 2^{r}$-cohomology classes $z_{*} \epsilon$ $H^{*}\left(X, Z / 2^{r}\right)$ correspond bijectively with homomorphisms

$$
\phi_{*}: \Omega_{*}^{P L}\left(X, Z / 2^{r}\right) \longrightarrow Z / 2^{r},
$$

which satisfy

( i ) $\phi_{*}(i([M, f] \cdot[N]))=\phi_{*}(i[M, f]) \cdot i n d e x(N) \in Z / 2^{s} \subset Z / 2^{r}$, where $[M, f] \in \Omega_{*}^{P L}\left(X, Z / 2^{s}\right),[N] \in \Omega_{*}^{P L}\left(p t, Z / 2^{s}\right), s \leqq r$.

(ii) $\phi_{*}\left(\rho_{2^{r}} \delta([P, F] \cdot[Q])\right)=\phi_{*}\left(\rho_{2^{r}} \delta[P, F]\right) \cdot \operatorname{index}(Q) \in Z / 2^{s} \subset Z / 2^{r}$, where $[P, F] \in \Omega_{*}^{P L}\left(X, Z / 2^{s}\right), Q \in \Omega_{*}^{P L}\left(p t, Z / 2^{s}\right), s \leqq r$.

The defining equation is

$$
\phi_{*}([M, f])=\left\langle\rho_{2^{r}} L(M) \cdot f^{*}\left(\sum_{i \geqq 0} z_{n-4 i}\right),[M]\right\rangle \in Z / 2^{r}
$$

\section{REFERENCES}

1. W. Browder, The Kervaire invariant, products, and Poincaré iransversality.

2. - Surgery on Simply Connected Manifolds, Springer-Verlag, 1972.

3. - The Kervaire invariant of framed manifolds and its generalization, Ann. of Math., 90 (1969), 157-186.

4. G. Brumfiel, I. Madsen and R. J. Milgram, PL characteristic classes and cobordism, Ann. of Math., 97 (1973), 82-159.

5. G. Brumfiel and R. J. Milgram,

6. G. Brumfiel and J. Morgan, Quadratic functions, the index modulo 8, and a $Z / 4-$ Hirzebruch formula, Topology

7. S. Gitler and J. Stasheff, The first exotic class of BF, Topology, 4 (1965), 257-266.

8. L. Jones, Patch spaces: a geometric representation for Poincaré spaces, Ann. of Math., 97 (1973), 306-343.

9. R. Kirby and L. Siebenmann, Some theorems on topological manifolds, ManifoldsAmsterdam 1970, Springer Lecture Notes 197.

10. N. Levitt, Poincaré duality cobordism, Ann. of Math., 96 (1972), 211-244.

11. N. Levitt and J. Morgan, Transversality structures and PL structures on spherical fibrations, Bull. Amer. Math. Soc., 78 (1972), 1064-1068.

12. I. Madsen and R. J. Milgram, On spherical fibre bundles and their PL reduction,

13. R. J. Milgram, Surgery with coefficients, Ann. of Math.

14. R. J. Milgram,

15. J. Morgan and D. Sullivan, The transversality characteristic class and linking cycles in surgery theory, Ann. of Math.

16. S. Papastavridas,

17. F. P. Peterson and H. Toda, On the structure of $H^{*}\left(B S F, Z_{p}\right)$, J. Math. Kyoto Univ., (1967), 113-121.

18. F. Quinn, Surgery on Poincaré and normal spaces, Bull. Amer. Math. Soc., 78 (1972), 262-267.

19. - $B_{T O P(n)}$ and the surgery obstruction, Bull. Amer. Math. Soc., 79 (1971), $596-600$.

20. $-B_{T O P(n)}$ and the surgery obstruction $I I$,

21. D. Ravenal, $A$ definition of exotic characteristic classes of spherical fibrations,

22. C. P. Rourke and D. Sullivan, On the Kervaire obstruction, Ann. of Math., 94 
(1971), 397-413.

23. D. Sullivan, Geometrichomotopy theory, mimeographed, Princeton University, 1967.

24. C. T. C. Wall, Poincaré complexes I, Ann. of Math., 86 (1967), 77-101.

25. - Surgery on Compact Manifolds, Academic Press, New York, 1971.

Received August 30, 1974.

STANFORD UNIVERSITY

AND

MIT 


\section{PACIFIC JOURNAL OF MATHEMATICS}

EDITORS

RICHARD ARENS (Managing Editor)

University of California

Los Angeles, California 90024

R. A. BEAUMONT

University of Washington

Seattle, Washington 98105

J. DugundjI

Department of Mathematics

University of Southern California

Los Angeles, California 90007

D. Gilbarg and J. Milgram

Stanford University

Stanford, California 94305

\section{ASSOCIATE EDITORS}

E. F. BeCKENBACH

B. H. NeUMaNN

F. WOLF

K. YoSHIDA

\section{SUPPORTING INSTITUTIONS}

UNIVERSITY OF BRITISH COLUMBIA

CALIFORNIA INSTITUTE OF TECHNOLOGY

UNIVERSITY OF CALIFORNIA

MONTANA STATE UNIVERSITY

UNIVERSITY OF NEVADA

NEW MEXICO STATE UNIVERSITY

OREGON STATE UNIVERSITY

UNIVERSITY OF OREGON

OSAKA UNIVERSITY

\author{
UNIVERSITY OF SOUTHERN CALIFORNIA \\ STANFORD UNIVERSITY \\ UNIVERSITY OF TOKYO \\ UNIVERSITY OF UTAH \\ WASHINGTON STATE UNIVERSITY \\ UNIVERSITY OF WASHINGTON \\ AMERICAN MATHEMATICAL SOCIETY \\ NAVAL WEAPONS CENTER
}




\section{Pacific Journal of Mathematics \\ Vol. 67, No. $1 \quad$ January, 1976}

Gregory Wayne Brumfiel and John W. Morgan, Homotopy theoretic

consequences of $N$. Levitt's obstruction theory to transversality for

spherical fibrations . ................................ 1

Jacob Burbea, Total positivity of certain reproducing kernels ........... 101

Wai-Mee Ching, The structure of standard $C^{*}$-algebras and their

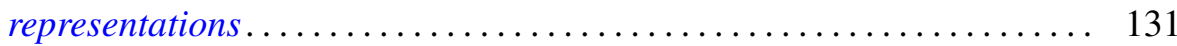

Satya Deo, The cohomological dimension of an $n$-manifold is $n+1 \ldots \ldots 155$

Masahiko Fujiwara and Masaki Sudo, Some forms of odd degree for which the Hasse principle fails ................................. 161

Mikihiro Hayashi, Smoothness of analytic functions at boundary points ... 171

Rebecca A. Herb, A uniqueness theorem for tempered invariant

eigendistributions ................................ 203

David Alan Legg, Orlicz space convergence of martingales of

Radon-Nikodým derivatives given a $\sigma$-lattice.................. 209

D. B. McAlister, v-prehomomorphisms on inverse semigroups......... 215

Bruno J. Mueller, Localization in fully bounded Noetherian rings . ........ 233

Donald J. Newman and A. R. Reddy, Rational approximation to $x^{n} \ldots \ldots .247$

Abraham Ziv, Inclusion relations between power methods of limitation.... 251 